\title{
Precision Measurement of the Neutron Spin Structure Function
}

\section{Piotr Zyla}

SLAC-R-623

Prepared for the Department of Energy

under contract number DE-AC02-76SF00515

Printed in the United States of America. Available from the National Technical Information Service, U.S. Department of Commerce, 5285 Port Royal Road, Springfield, VA 22161. 


\title{
Precision Measurement of the Neutron Spin Structure Function.
}

\author{
A Dissertation Submitted to \\ the Temple University Graduate Board \\ in Partial Fulfillment of the Requirements for the Degree \\ DOCTOR OF PHILOSOPHY
}

by

Piotr A. Zyla

January, 1998 


\section{ABSTRACT}

The SLAC E154 experiment measured the neutron spin structure function $g_{1}^{n}\left(x, Q^{2}\right)$. Scattering the $48.3 \mathrm{GeV}$ longitudinally polarized electrons off the longitudinally polarized ${ }^{3}$ He target allowed us to extend (in comparison with the previous SLAC E142 measurement) the kinematic range to $0.014 \leq x \leq$ 0.7 and $1 \mathrm{GeV}^{2} \leq \mathrm{Q}^{2} \leq 17 \mathrm{GeV}^{2}$. We report the integral over the measured region $\int_{0.014}^{0.7} d x g_{1}^{n}(x)=-0.036 \pm 0.004$ (stat.) \pm 0.005 (syst.) at the average $Q^{2}=5 \mathrm{GeV}^{2}$ of the experiment. We found large and strongly $x$ dependent values of $g_{1}^{n}$ at low $x$ that call into question the traditional Regge theory method for extrapolating to $x=0$ needed for the evaluation of QCD sum rules. Within perturbative QCD we performed the next to leading order global analysis of all polarized DIS structure function measurements. It explains the perturbative origin of the observed low $x$ behavior. Using this next to leading order parametrization we evolve the neutron $g_{1}^{n}$ and proton $g_{1}^{p}$ experimental data to a common value of $Q^{2}=5 \mathrm{GeV}^{2}$, evaluate their integrals and confirm the Bjorken sum rule. 


\section{TABLE OF CONTENTS}

Page

ABSTRACT $\ldots \ldots \ldots \ldots \ldots \ldots$ iii

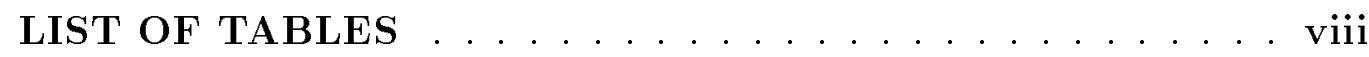

LIST OF FIGURES $\ldots \ldots \ldots \ldots \ldots \ldots \ldots \ldots$

\section{CHAPTER}

1 INTRODUCTION TO SPIN-DEPENDENT DEEP INELASTIC SCATTERING ............... 1

1.1 Historical background . . . . . . . . . . . . . . 1

1.2 Structure functions . . . . . . . . . . . . 3

1.3 Virtual photon-nucleon asymmetries . . . . . . . . 9

1.4 Models of the $\mathrm{He}^{3}$ nucleus . . . . . . . . . . . . . 12

1.5 Partonic interpretation . . . . . . . . . . . 13

1.6 QCD improved parton model . . . . . . . . . 18

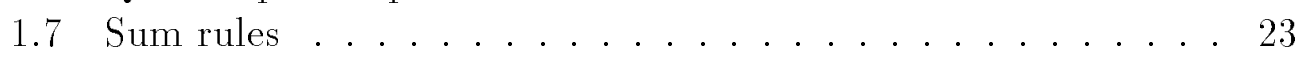

1.7.1 Helicity sum rule . . . . . . . . . . . . . . 23

1.7 .2 Bjorken sum rule . . . . . . . . . . . . 26

1.7 .3 Ellis-Jaffe sum rule . . . . . . . . . . . . 28

1.7.4 Sum rules for $g_{2} \ldots \ldots \ldots \ldots \ldots$

1.8 Traditional evaluation of sum rules from measurements . . . . 31

1.8.1 Evolution in $Q^{2} \ldots \ldots \ldots \ldots \ldots \ldots$

1.8.2 The low $x$ extrapolation . . . . . . . . . . 32

1.8.3 The high $x$ extrapolation . . . . . . . . . 33 
1.9 Summary of polarized DIS experiments . . . . . . . . . 34

2 EXPERIMENTAL APPARATUS ........... 41

2.1 SLAC polarized source . . . . . . . . . . . 42

2.2 Beam acceleration and transport ........... 46

2.3 Beam parameters and monitoring .................... 47

2.3.1 Energy .................... 47

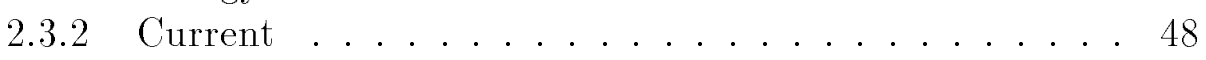

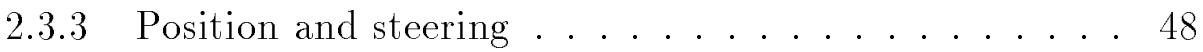

2.3.4 Spin precession ............... 50

2.3.5 Helicity reversal ............... 51

2.4 Møller polarimeter . . . . . . . . . . . . . 53

2.4.1 Møller asymmetry . . . . . . . . . . . 53

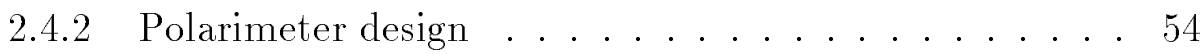

2.4.3 Foil polarization measurement .......... 56

2.4.4 Beam polarization results ........... 58

2.4.5 Systematic uncertainties ............ 59

2.4.6 Beam energy scan and beam helicity determination . . 60

2.5 Polarized ${ }^{3}$ He target ................. 63

2.5.1 Optical pumping and spin exchange........ 63

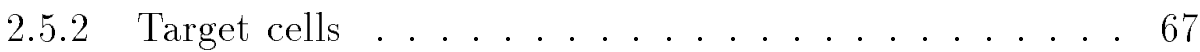

2.5.3 Target setup ................. 70

2.5.4 Polarimetry and polarization results........ 72

2.5.5 Target polarization direction ........... 76

2.6 Magnetic spectrometers . . . . . . . . . . 77

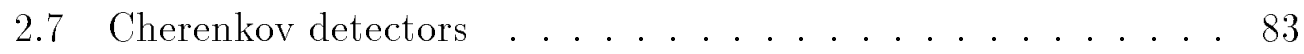

2.7.1 Cherenkov radiation ............. 84

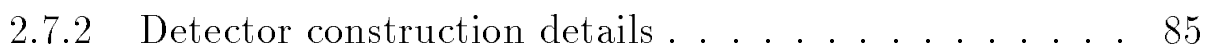

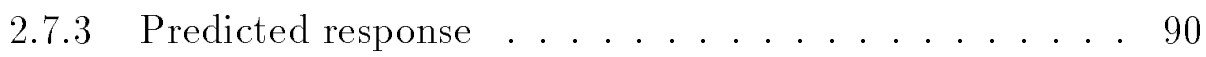

2.7.4 Cherenkov electronics . . . . . . . . . . . 92

2.8 Scintillator hodoscopes . . . . . . . . . . . . . 92

2.9 Electromagnetic shower counter ............. 94

2.10 Data acquisition (DAQ) . . . . . . . . . 96

3 DATA ANALYSIS AND RESULTS ........... 100

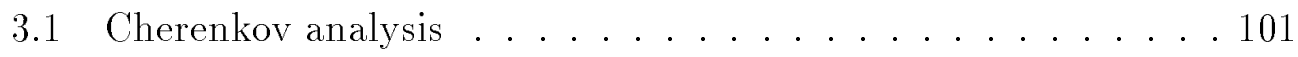

3.2 Energy determination: Shower analysis . . . . . . . . 105 
3.3 Momentum determination: Tracking . . . . . . . . . 111

3.4 Event analysis: Run selection and electron identification . . . 117

3.4.1 Run selection . . . . . . . . . . . . . . 117

3.4.2 Event selection, electron id and kinematics . . . . . . 118

3.5 Asymmetry analysis . . . . . . . . . . . . . . 122

3.6 Background subtraction . . . . . . . . . . . . 124

3.6.1 Pion background . . . . . . . . . . . . . 125

3.6.2 Charge symmetric background . . . . . . . . . . . 127

3.7 Rate dependence . . . . . . . . . . . . . . . . 130

3.7.1 Pulse fiction method . . . . . . . . . . . . 132

3.7.2 Momentum and energy rate dependence . . . . . . . 135

3.7.3 Detector rate dependence . . . . . . . . . . . 138

3.7 .4 Asymmetry correction . . . . . . . . . . . . . . 144

3.8 Electroweak correction . . . . . . . . . . . . . . 145

3.9 Radiative corrections . . . . . . . . . . . . . 146

3.9.1 Internal radiative corrections . . . . . . . . . . 146

3.9 .2 External radiative corrections . . . . . . . . . . . 151

3.9.3 Radiative corrections results . . . . . . . . . 152

3.10 Dilution factor . . . . . . . . . . . . . . 154

3.10 .1 Model calculation . . . . . . . . . . . . . . . . 154

3.10 .2 Measurement . . . . . . . . . . . . . . 155

3.10 .3 Comparison and results . . . . . . . . 156

3.11 Asymmetry results for ${ }^{3} \mathrm{He} \ldots \ldots \ldots \ldots \ldots$. . . . . . . 157

3.12 Determination of the neutron structure functions $\ldots \ldots . .157$

3.13 Discussion of the results . . . . . . . . . . 166

4 GLOBAL FIT $\ldots \ldots \ldots \ldots \ldots \ldots \ldots \ldots \ldots$

4.1 Factorization theorem . . . . . . . . . . 177

4.2 Evolution . . . . . . . . . . . . . . . . 179

4.3 Initial distributions . . . . . . . . . . . . . . . 181

4.3.1 High $x$ asymptotics . . . . . . . . . . . . . 182

4.3 .2 Low $x$ phenomenology . . . . . . . . . . . . 187

4.3 .3 Shape of distributions . . . . . . . . . . . . . 191

4.4 Fitting procedure . . . . . . . . . . . . . . 192

4.5 Mellin transform technique . . . . . . . . . . . . . 195

4.6 Dependence on the renormalization and factorization schemes 197

4.7 Analysis of errors . . . . . . . . . . . . . . 202 
4.7.1 Statistical errors . . . . . . . . . . . . . . 202

4.7 .2 Systematic errors . . . . . . . . . . . . . . . . 203

4.7.3 Theoretical uncertainties . . . . . . . . . . . . 205

4.8 Results . . . . . . . . . . . . . . . . . . 205

4.9 Revised low $x$ extrapolation $\ldots \ldots \ldots \ldots . \ldots \ldots 213$

5 SUMMARY AND OUTLOOK $\ldots \ldots \ldots \ldots \ldots$

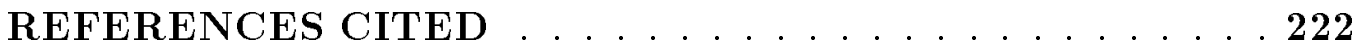

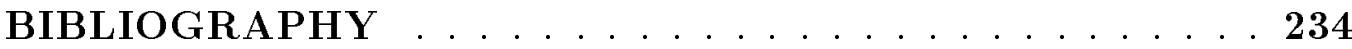

\section{APPENDIX}

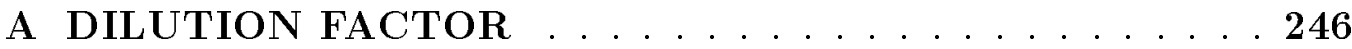

A.1 Modeling dilution factor . . . . . . . . . . . 24 247

A.1.1 Radiative corrections . . . . . . . . . . . . . 248

A.1.2 Helium contribution . . . . . . . . . . . . . . 249

A.1.3 Nitrogen contribution . . . . . . . . . . . . . 249

A.1.4 Glass compounds . . . . . . . . . . . . . 2 250

A.1.5 Numerical results . . . . . . . . . . . . . . . . . 251

A.1.6 Error analysis . . . . . . . . . . . . . . 2 251

A.1.7 Summary . . . . . . . . . . . . . . 255

A.2 Measurement . . . . . . . . . . . . . 257

A.2.1 Experimental procedure . . . . . . . . . . 257

A.2.2 Rate dependence correction . . . . . . . . . . . 258

A.2.3 Background subtraction . . . . . . . . . . . . . 259

A.2.4 Run selection . . . . . . . . . . . . . 261

A.2.5 Error analysis . . . . . . . . . . . . . . . 262

A.3 Comparison and final results . . . . . . . . . . 264

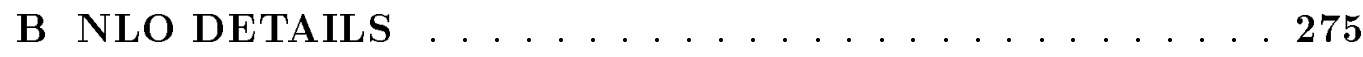

B.1 Wilson coefficients and the splitting

functions in the $x$-space . . . . . . . . . . 2 275

B.2 Evolution in the $\mathrm{n}$-th moment Mellin space . . . . . . . 279 


\section{LIST OF TABLES}

1.1 Kinematic Variables for Inclusive Electron-Proton Scattering. . 5

1.2 Integrals of $g_{1}^{n, p, d}$ From CERN, SLAC and DESY Experiments. 36

2.1 Measured Longitudinal Beam Polarization for Different Running Periods. . . . . . . . . . . . 60

2.2 Relative Systematic Error Contributions to the Beam Polarization Measurement. . . . . . . . . . . . . 60

2.3 Chemical Composition of Corning 1720 Glass by Weight. . . . 67

2.4 E154 Target Cells. . . . . . . . . . . . . . . . . . 69

2.5 The Cherenkov Detector Characteristics. . . . . . . . . 87

2.6 Characteristics of Hamamatsu R1584 PMTS. . . . . . . . . . 89

2.7 Geometrical Parameters of E154 Hodoscopes. . . . . . . . . . 93

3.1 Cherenkov Response to Electrons. . . . . . . . . . . . . 104

3.2 Radiative Corrections and Their Systematic Errors. . . . . . 153

3.3 Results for the ${ }^{3} \mathrm{He}$ Asymmetries $A_{\|}$and $A_{\perp} \ldots \ldots \ldots \ldots 158$

3.4 Averaged Results of the Two Analyses on ${ }^{3} \mathrm{He}$ Asymmetries $A_{\|}$and $A_{\perp} \ldots \ldots \ldots \ldots \ldots \ldots \ldots$

3.5 The Spin Dependent Structure Function $g_{1}^{n}$ and the Virtual Photon-Nucleon Asymmetry $A_{1}^{n} \ldots \ldots \ldots \ldots \ldots 1$

3.6 The Spin Dependent Structure Function $g_{2}^{n}$ and the Virtual Photon-Nucleon Asymmetry $A_{2}^{n}$. . . . . . . . . . . 162

3.7 Results on $A_{1}^{n}$ and $g_{1}^{n}$ at the Measured $Q^{2}$, Along With $g_{1}^{n}$ Evaluated at $Q^{2}=5(\mathrm{GeV})^{2}$ Assuming That the Ratio $g_{1}^{n} / F_{1}^{n}$ Scales With $Q^{2} \ldots \ldots \ldots \ldots \ldots \ldots$

3.8 Contributions to the Systematic Error of $g_{1}^{n}$ for Every $x$ Bin and of the Integral Over the Measured Range. . . . . . . . . 164 
4.1 Fitted Values of the Free Parameters in eq. (4.42) in the $\overline{\mathrm{MS}}$ and $\mathrm{AB}$ Schemes. . . . . . . . . . . . . 206

4.2 First Moments of the Polarized Parton Distributions and Structure Functions of the Proton, Neutron, and Deuteron in the $\overline{\mathrm{MS}}$ and $A B$ Schemes Evaluated at $Q^{2}=5 \mathrm{GeV}^{2} \ldots \ldots 208$

4.3 E154 Results for $g_{1}^{n}$ Evolved to $\left\langle Q^{2}\right\rangle=5 \mathrm{GeV} 2$ Assuming $g_{1} / F_{1}$ is Independent of $Q^{2}$ and According to Eq. (4.71). . . . 211

4.4 Results for $g_{1}^{n}$ Evolved to $\left\langle Q^{2}\right\rangle=5 \mathrm{GeV}^{2}$ According to Eq. (4.71).213

A.1 Glass Compounds Density $n(\mathrm{~mol} / \mathrm{cc})$. . . . . . . . . 250

A.2 Propagation of $x$ Independent Relative Errors. . . . . . . . . 254

A.3 Propagation of $x$ Dependent Relative Errors. . . . . . . . 256

A.4 Model Results for the Target 'Picard', 2.75․ . . . . . . . 256

A.5 Relative $x$ Independent Errors For Measured $f$. . . . . . . 263

A.6 Measured $(\delta f / f)(x)$ Errors in \% and $f$ Results for 'Picard',

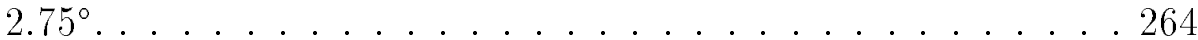

A.7 Comparison of Some Characteristics of the Model and Experiment and the Average Dilution Factor Error as Functions of x Bin. . . . . . . . . . . . . . 268

A.8 Comparison of Some Characteristics of the Model and Experiment and the Average Dilution Factor Error for Each Target and Spectrometer. . . . . . . . . . . . 269

A.9 Results for 'Dave', 'Riker', 'Bob', 'SMC' and 'Generals', 5.5 . . 269

A.10 Results for 'Dave', 'Riker', 'Bob', 'SMC' and 'Generals', 2.75'. 270

A.11 Results for 'Hermes','Prelims', 'Chance' and 'Picard', 2.75 . . . 270

A.12 Results for 'Hermes', 'Prelims', 'Chance' and 'Picard', 5.5 . . . 271 


\section{LIST OF FIGURES}

1.1 Tree level diagram for DIS lepton-hadron scattering. . . . . . . 4

1.2 Proton data for $x g_{1}^{p}(x) \ldots \ldots \ldots . \ldots . \ldots 38$

1.3 Neutron data for $x g_{1}^{n}(x) \ldots \ldots \ldots$. . . . . . . . 39

2.1 SLAC polarized source setup for SLC runs. . . . . . . . . . 43

2.2 The energy level structure of strained GaAs. . . . . . . . 45

2.3 Top and side views of the E-154 Møller Polarimeter. . . . . . . 55

2.4 Measured longitudinal beam polarization versus E154 run num-

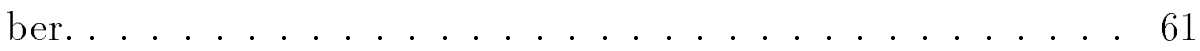

2.5 Measured beam polarization versus the nominal A-line beam energy. . . . . . . . . . . . . . . 62

2.6 Optical pumping of Rubidium vapor by circularly polarized light. .................... 64

2.7 Polarization of the target cell Picard during the run. . . . . 66

2.8 Schematic of the E154 target. . . . . . . . . . . 71

2.9 AFP-NMR signals of ${ }^{3} \mathrm{He}$ and water. . . . . . . . . 73

2.10 EPR frequency measurement for both polarization directions for the target cell Picard. . . . . . . . . . . . 75

2.11 E154 kinematic coverage in $x$ and $Q^{2} \ldots \ldots \ldots 78$

2.12 Differential cross section for the ${ }^{3}$ He target. . . . . . . . . 79

2.13 Schematic layout of the E154 spectrometers. . . . . . . . . 80

2.14 Optics of the $2.75^{\circ}$ and $5.5^{\circ}$ spectrometers in the bend and non-bend planes. . . . . . . . . . . . . 82

2.15 Original and remeasured reflectivity of the mirrors. . . . . . 88

2.16 The VME-based Data Acquisition system for ESA. . . . . . 97

3.1 An example of a Cherenkov signal digitized by FADC. . . . . 102 
3.2 The time resolution of unsynchronized and TDC synchronized

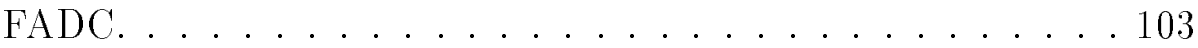

3.3 Typical Cherenkov response to electrons and pions. . . . . . . 104

3.4 The pulse height as a function of the difference between the leading and trailing edge times for different discriminator levels. 108

3.5 Electron and pion shower energy shapes. . . . . . . . . . 109

3.6 The cluster position as a function of the side to central block energy ratio. . . . . . . . . . . . . . 109

3.7 Distribution of the shower shape variable $\chi$ for electrons and

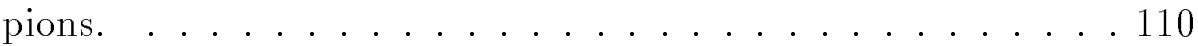

3.8 Tracking inefficiencies for two spectrometers determined by the Monte Carlo method. . . . . . . . . . . . . . . . 115

3.9 Average momentum and angular resolution in the bend and non-bend planes. . . . . . . . . . . . 116

3.10 Cuts influence on $E / p$ distribution. . . . . . . . . . . . 121

3.11 Pion contamination in the $x=0.0152$ bin. . . . . . . . . 125

3.12 Pion contamination. . . . . . . . . . . 126

3.13 Pion asymmetry. . . . . . . . . . . . . 127

3.14 Positron contamination. . . . . . . . . . . . 128

3.15 Positron asymmetry. . . . . . . . . . . . . . . 129

3.16 ADC signals from spill 1 and 2 merged to the double spill rate. 133

3.17 FADC signals from spill 1 and 2 merged to the double spill rate. 134

3.18 The distribution of the time difference between single and double rates tracks. . . . . . . . . . . . . . . 135

3.19 The distribution of distance between single and double rates tracks at the front hodoscopes and at the shower counter. . . . 136

3.20 The relative momentum and energy changes as a function of Bjorken $x$ in $2.75^{\circ}$ spectrometer when the rate is doubled. . . 137

3.21 The relative momentum and energy changes, when the rate is doubled, as a function of Bjorken $x$ in $2.75^{\circ}$ spectrometer for all target cells. . . . . . . . . . . . . . . 139

3.22 The detector rate dependence coefficients $\alpha_{\text {cher }}, \alpha_{\text {hodo }}, \alpha_{\text {shw }}$ and $\alpha_{\text {total }}$ for the target cell Picard, $2.75^{\circ}$ spectrometer. . . . 140

3.23 Rate dependence coefficients $\alpha$ for the $2.75^{\circ}$ and $5.5^{\circ}$ spectrometers. . . . . . . . . . . . . . . . . 142

3.24 Rate dependence of different electron definitions. . . . . . . . 143 
3.25 The unpolarized Born cross section and various radiative tails in the $2.75^{\circ}$ spectrometer. . . . . . . . . . . . . . 150

3.26 The polarized radiative tails in the $2.75^{\circ}$ spectrometer. . . . . 151

3.27 Comparison of the theoretical with experimental dilution factors. 156

3.28 The structure function $x g_{1}^{n}$ measured in the $2.75^{\circ}$ and $5.5^{\circ}$ spectrometers. . . . . . . . . . . . 165

3.29 The structure function $x g_{2}^{n}$ measured in the $2.75^{\circ}$ and $5.5^{\circ}$ spectrometers. . . . . . . . . . . . 166

3.30 The structure function $g_{1}^{n}$ evaluated at $Q^{2}=5 \mathrm{GeV}^{2}$. . . 167

3.31 The E154 results on the structure function $x g_{1}^{n}$ compared to

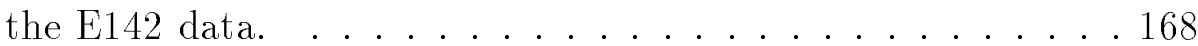

3.32 Comparison of the E154 results to the E142 and E143 data at

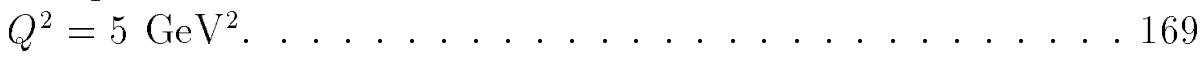

3.33 Comparison of the E154 and SMC data. . . . . . . . 170

3.34 Two fits to the low $x$ data of E154. . . . . . . . . . 171

3.35 The integral of $g_{1}^{n}(x)$ from $x=x_{\min }$ to $x=1$ plotted as a function of $x_{\text {min }} \ldots \ldots \ldots \ldots \ldots \ldots \ldots$

3.36 The integral of $\left(g_{1}^{n}-g_{1}^{p}\right)(x)$ from $x=x_{\min }$ to $x=1$ plotted as a function of $x_{\text {min }} \ldots \ldots \ldots \ldots \ldots$. . . . . . . . . . . . . . .

4.1 Change of the partonic probability due to the radiation of a gluon. . . . . . . . . . . . . . . 181

4.2 Leading-order diagram for the amplitude $\psi(x)$ at $x \sim 1 \ldots . .183$

4.3 Kinematics coverage of the polarized DIS data. . . . . . . . 194

4.4 Distributions of the first moments of the parton densities in the $\overline{\mathrm{MS}}$ scheme due to the 'statistical' randomization of the $g_{1}$ data, as described in the text. ............. 204

4.5 The structure functions $x g_{1}^{p}$ and $x g_{1}^{n}$ at $Q^{2}=5 \mathrm{GeV}^{2} \ldots 207$

4.6 The structure function $x g_{1}^{n}$ evolved to $Q^{2}=5 \mathrm{GeV}^{2}$ using our $\overline{\mathrm{MS}}$ parametrization, and assuming that $g_{1}^{n} / F_{1}^{n}$ is independent of $Q^{2}$. . . . . . . . . . . . . . . . . . 209

4.7 Evolution of the ratios $g_{1} / F_{1}$ for the proton and the neutron. . 212

4.8 The contributions to the structure function $g_{1}^{n}$ of the neutron from the valence quarks and from the sea quarks and gluons. . 215

4.9 The $g_{1}^{p}$ structure function at $Q^{2}=5 \mathrm{GeV}^{2}$. . . . . . . 216

A.1 Typical $f$ model result. . . . . . . . . . . . . . . 252

A.2 Measured result for target 'Picard', $2.75^{\circ} \ldots \ldots . \ldots 262$ 
A.3 Target 'Dave', $2.75^{\circ}$ and $5.5^{\circ} \ldots \ldots . \ldots . \ldots 267$

A.4 Target 'Riker', $2.75^{\circ}$ and $5.5^{\circ} \ldots \ldots . \ldots . \ldots 267$

A.5 Target 'Bob', $2.75^{\circ}$ and $5.5^{\circ} \ldots \ldots . \ldots . \ldots . \ldots 271$

A.6 Target 'SMC', $2.75^{\circ}$ and $5.5^{\circ}$. . . . . . . . . . . . 272

A.7 Target 'Generals', $2.75^{\circ}$ and $5.5^{\circ} \ldots \ldots \ldots . \ldots . \ldots . \ldots 272$

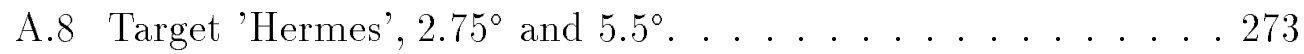

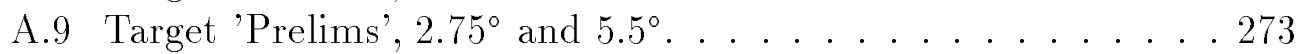

A.10 Target 'Chance', $2.75^{\circ}$ and $5.5^{\circ} \ldots \ldots . \ldots 274$

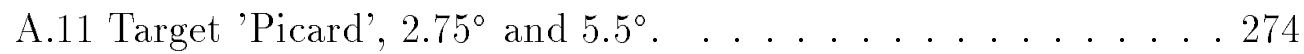

B.1 Integration contours of the Mellin inverse transform. . . . . 285

B.2 Comparison of NLO partonic distributions evolved from $Q_{\circ}^{2}=$ $0.34 \mathrm{GeV}^{2}$ to $Q^{2}=100 \mathrm{GeV}^{2}$ with the output of the GRSV code $[131,193] \ldots \ldots \ldots \ldots 1$ 


\section{CHAPTER 1}

\section{INTRODUCTION TO SPIN-DEPENDENT DEEP INELASTIC SCATTERING}

\section{$1.1 \quad$ Historical background}

Since the beginnings of ancient Greek philosophy, natural sciences have been in search for the atoms of Democritus, the indivisible building blocks from which more complicated objects are built. Physics considers the question of the elementary objects that constitute matter. It has only been in the sixties that, based on hadron spectroscopy, Gell-Mann [1] and Zweig [2] suggested that quarks are the fundamental building blocks of hadrons. The quark model, based on $\mathrm{SU}(3)_{\text {flavor, }}$, was formulated, and the search for its dynamics began. The simplest experimental method of probing the hadrons (protons) seemed to be with a beam of structureless leptons (electrons). The first experiments, called Deep Inelastic Scattering (DIS), were performed at 
SLAC's End Station A in the late 1960's [3]. They revealed the approximate Bjorken scaling [4] of the structure functions in the large momentum transfer $Q^{2}$. One of the easiest ways of understanding this feature was to assume that the electrons scattered off almost free pointlike partons, as proposed by Bjorken and Paschos [5] and Feynman [6]. Thus the underlying dynamics should exhibit weaker coupling at shorter distances (larger momenta). At that time none of the proposed quantum field theories for quarks possessed that property. The long waited Yang-Mills [7] non-Abelian gauge theory was examined, and soon t'Hooft [8], Gross and Wilczek [9], and Politzer [10] reported its property of asymptotic freedom. In the meantime Han and Nambu [11] had suggested the color quantum number for quarks to resolve several difficulties of the quark model. Fritzsch and Gell-Mann [12] proposed the $\mathrm{SU}(3)_{\text {color }}$ group for the symmetry of the non-Abelian gauge theory. Quantum Chromodynamics (QCD) was born, and the quarks were identified with partons. We were reminded of the importance of the early DIS experiments at SLAC when experimenters Jerome I. Friedman, Henry W. Kendall and Richard E. Taylor received the 1990 Nobel Prize in Physics [13].

The pioneering DIS experiments probed the momentum distribution of partons within the nucleon. The polarized experiments that dealt with the partonic spin distribution were performed by Yale-SLAC group [14, 15] soon after them. This work received renewed attention when EMC [16] extended the SLAC measurements on the proton to smaller $x$ values and concluded that the quarks carry very little of the nucleon spin, in contradiction with 
the expectation from naive quark models. Since then much more data have been obtained by SLAC [17, 18, 19], CERN [20, 21] and DESY [22].

Our experiment E154 took place at SLAC's End Station A in OctoberNovember 1995. By scattering longitudinally polarized electrons from a longitudinally and transversely polarized ${ }^{3} \mathrm{He}$ targets, we measured the spin structure functions of the neutron $g_{1}^{n}\left(x, Q^{2}\right)$ and $g_{2}^{n}\left(x, Q^{2}\right)$. The high statistical precision and the broad kinematic range of the data gives us a better insight into the dynamics of quarks and gluons within the nucleon.

\subsection{Structure functions}

To explore the internal structure of the nucleon it is necessary to use a probe which has a wavelength smaller than the size of the nucleon. Since the wavelength of a particle is proportional to the inverse of its momentum, the probe must be very energetic. It is also convenient if the probe itself is structureless, say a lepton. In addition, if we want to look at the spin distribution within the nucleon both, as we shall see later, the object and the probe need to have a definite polarization. Let's consider a typical fixed target DIS experiment in which the polarized electrons scatter on a polarized proton target. In the lowest order in the electromagnetic coupling one virtual photon is exchanged in that process. This is shown in figure 1.1. The incident electron has an initial four-momentum $k^{\mu}=(E, \vec{k})$ and a polarization fourvector $^{1} s^{\mu}$. The electron scatters with respect to $\vec{k}$ through the lab angle

\footnotetext{
${ }^{1}$ In the rest frame the polarization four-vector $s^{\mu}$ has the form $s^{\mu}=(0, \vec{s})$ where $\vec{s}$ is a polarization. It is orthogonal to the momentum, $s \cdot p=0$, and normalized, $s^{2}=-1$.
} 


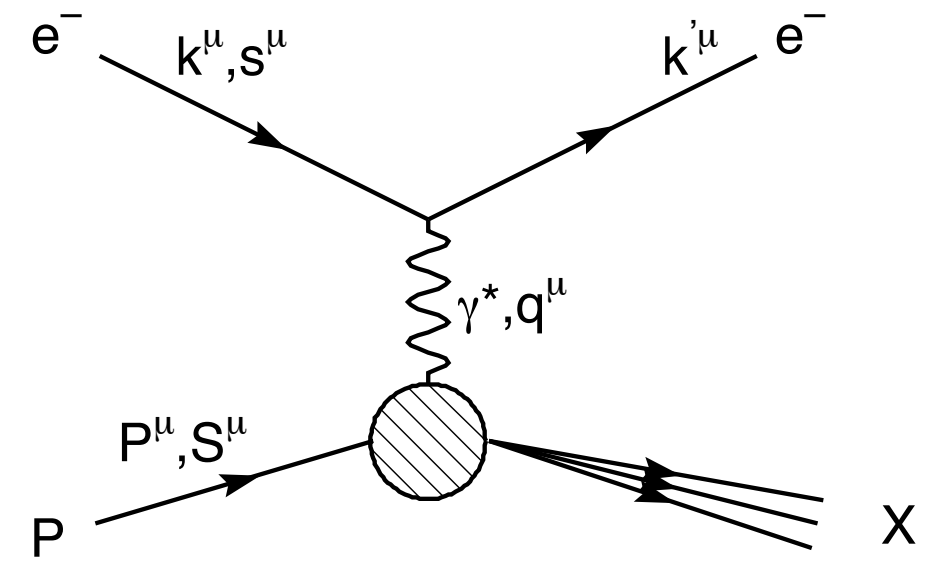

Figure 1.1: Tree level diagram for DIS lepton-hadron scattering.

$\theta$ and has final momentum $k^{\prime \mu}=\left(E^{\prime}, \overrightarrow{k^{\prime}}\right)$. The exchanged virtual photon carries momentum $q^{\mu}=\left(k-k^{\prime}\right)^{\mu}$. The proton has momentum $P^{\mu}=(M, \overrightarrow{0})$ in the lab frame and polarization $S^{\mu}=(0, \vec{S})$. The final hadronic states $X$ with the same invariant mass $W^{2}=(P+q)^{2}$ are not detected. The process is described by two independent kinematic invariants, $Q^{2}=-q^{2}$ and $\nu=P \cdot q / M\left(=E-E^{\prime}\right.$ in the lab) or equivalently by $Q^{2}$ and $x=\frac{Q^{2}}{2 M \nu}$. From the experimental point of view it is convenient to use the lab frame and work with the measurable quantities, $E^{\prime}$ and $\theta$. The kinematic variables are summarized in table 1.1. The differential cross section for the considered process, calculated from the leading order diagram in fig. 1.1, can be written as $[23]$

$$
\frac{d^{2} \sigma}{d \Omega d E^{\prime}}=\frac{4 \alpha^{2}}{Q^{4}} \frac{E^{\prime}}{E} L_{\mu \nu} W^{\mu \nu}
$$


Table 1.1: Kinematic Variables for Inclusive Electron-Proton Scattering.

\begin{tabular}{|c|c|c|c|}
\hline Symbol & Definition & Lab frame & Description \\
\hline$E$ & & & energy of incident $e$ \\
\hline$\vec{k}$ & & & momentum of incident $e$ \\
\hline$k^{\mu}$ & $(E, \vec{k})$ & & 4-momentum of incident $e$ \\
\hline$s^{\mu}$ & & & polariz. 4-vector of incident $e$ \\
\hline$E^{\prime}$ & & & energy of scattered $e$ \\
\hline $\overrightarrow{k^{\prime}}$ & & & momentum of scattered $e$ \\
\hline$k^{\prime \mu}$ & $\left(E^{\prime}, \overrightarrow{k^{\prime}}\right)$ & & 4-momentum of scattered $e$ \\
\hline$m$ & & & mass of $e$ \\
\hline$\theta$ & $\arccos \left(\frac{\vec{k} \cdot \overrightarrow{k^{\prime}}}{|\vec{k}|\left|\overrightarrow{k^{\prime}}\right|}\right)$ & & scattering angle of $e$ \\
\hline$M$ & & & mass of proton \\
\hline$P^{\mu}$ & & $(M, 0)$ & 4-momentum of proton \\
\hline$S^{\mu}$ & & $(0, \vec{S})$ & polariz. 4-vector of proton \\
\hline$q^{\mu}$ & $\left(k-k^{\prime}\right)^{\mu}$ & $(\nu, \vec{q})$ & 4-momentum transfer \\
\hline$Q^{2}$ & $-q^{2}$ & $4 E E^{\prime} \sin ^{2}(\theta / 2)$ & 4-mom. transfer squared \\
\hline$\nu$ & $P \cdot q / M$ & $E-E^{\prime}$ & energy transfer \\
\hline$x$ & & $\frac{4 E E^{\prime} \sin ^{2}(\theta / 2)}{2 M\left(E-E^{\prime}\right)}$ & Bjorken $x$ \\
\hline
\end{tabular}

where $\alpha$ is the fine structure constant and $L_{\mu \nu}$, and $W^{\mu \nu}$ are leptonic and hadronic tensors, respectively. The leptonic part describes matrix elements of currents of elementary particles and has the form

$$
\begin{aligned}
L_{\mu \nu} & =2\left[k_{\mu} k_{\nu}^{\prime}+k_{\nu} k_{\mu}^{\prime}-g_{\mu \nu}\left(k \cdot k^{\prime}-m^{2}\right)\right]+2 i m \epsilon_{\mu \nu \rho \sigma} q^{\rho} s^{\sigma} \\
& \equiv L_{\mu \nu}^{S}+i L_{\mu \nu}^{A} .
\end{aligned}
$$

The part of $L_{\mu \nu}$ symmetric with respect to the interchange $\mu \leftrightarrow \nu$ is denoted by $L_{\mu \nu}^{S}$ and the antisymmetric part by $L_{\mu \nu}^{A}$. Note that only the antisymmetric 
part contains the electron polarization $s^{\mu}$. The tensor $W_{\mu \nu}$ is the Fourier transform of the correlator of unknown hadronic currents,

$$
W_{\mu \nu}=\frac{1}{2 \pi M} \int d^{4} x e^{i q \cdot x}\left\langle P, S\left|\left[J_{\mu}(x), J_{\nu}(0)\right]\right| P, S\right\rangle .
$$

We can use Lorentz invariance and impose the symmetry requirements of time reversal invariance, parity and current conservation, and hermiticity to parametrize it in terms of the structure functions $F_{1}, F_{2}, g_{1}$ and $g_{2}$. Decomposing $W_{\mu \nu}$ into symmetric and antisymmetric tensors,

$$
W_{\mu \nu}=W_{\mu \nu}^{S}+i W_{\mu \nu}^{A},
$$

we obtain

$$
W_{\mu \nu}^{S}=\left(\frac{q_{\mu} q_{\nu}}{q^{2}}-g_{\mu \nu}\right) \frac{F_{1}\left(x, Q^{2}\right)}{M}+\frac{1}{M^{2}}\left(P_{\mu}-\frac{P \cdot q}{q^{2}} q_{\mu}\right)\left(P_{\nu}-\frac{P \cdot q}{q^{2}} q_{\nu}\right) \frac{F_{2}\left(x, Q^{2}\right)}{\nu},
$$

and

$$
W_{\mu \nu}^{A}=M \epsilon_{\mu \nu \rho \sigma} q^{\rho} S^{\sigma} \frac{g_{1}\left(x, Q^{2}\right)}{M^{2} \nu}+\frac{1}{M} \epsilon_{\mu \nu \rho \sigma} q^{\rho}\left(P \cdot q S^{\sigma}-S \cdot q P^{\sigma}\right) \frac{g_{2}\left(x, Q^{2}\right)}{M \nu^{2}}
$$

We note again that only the antisymmetric part contains the proton polarization $S^{\mu}$. Since

$$
L_{\mu \nu} W^{\mu \nu}=L_{\mu \nu}^{S} W^{S \mu \nu}-L_{\mu \nu}^{A} W^{A \mu \nu},
$$

we need to polarize both the electron beam and the nucleon target to measure the spin structure functions $g_{1}$ and $g_{2}$.

Let us now consider longitudinally polarized electrons along ( $\uparrow$ ) or opposite $(\downarrow)$ to the direction of the beam, with the nucleon target polarized 
longitudinally $(\Uparrow, \Downarrow)$ or transversely $(\Rightarrow)$ to the beam direction. The differential cross sections have the form

$$
\frac{d^{2} \sigma^{\downarrow \Uparrow}}{d \Omega d E^{\prime}}+\frac{d^{2} \sigma^{\uparrow \Uparrow}}{d \Omega d E^{\prime}}=\frac{8 \alpha^{2}}{Q^{4}} E^{\prime 2}\left[2 \sin ^{2}\left(\frac{\theta}{2}\right) \frac{F_{1}\left(x, Q^{2}\right)}{M}+\cos ^{2}\left(\frac{\theta}{2}\right) \frac{F_{2}\left(x, Q^{2}\right)}{\nu}\right]
$$

and

$$
\frac{d^{2} \sigma^{\downarrow \Uparrow}}{d \Omega d E^{\prime}}-\frac{d^{2} \sigma^{\uparrow \Uparrow}}{d \Omega d E^{\prime}}=\frac{4 \alpha^{2}}{Q^{2}} \frac{E^{\prime}}{E \nu}\left[\frac{\left(E+E^{\prime} \cos \theta\right)}{M} g_{1}\left(x, Q^{2}\right)-2 x g_{2}\left(x, Q^{2}\right)\right]
$$

for the longitudinally polarized electrons and target and

$$
\frac{d^{2} \sigma^{\downarrow \Rightarrow}}{d \Omega d E^{\prime}}-\frac{d^{2} \sigma^{\uparrow \Rightarrow}}{d \Omega d E^{\prime}}=\frac{4 \alpha^{2}}{Q^{2}} \frac{E^{\prime 2}}{E} \frac{\sin \theta}{M \nu}\left[g_{1}\left(x, Q^{2}\right)+2 \frac{E}{\nu} g_{2}\left(x, Q^{2}\right)\right]
$$

for the longitudinally polarized electrons and transversely polarized target. The longitudinal asymmetry, see eq. (1.9), is dominated by the structure function $g_{1}$, as $g_{2}$ is suppressed by a factor $\sim \frac{M}{E} \approx 0.02$ for a $E=50 \mathrm{GeV}$ beam. On the other hand the transverse asymmetry, see eq. (1.10), is primarily sensitive to $g_{2}$.

In polarized experiments one usually measures asymmetries rather than cross sections. The raw asymmetries are computed from the detector rates according to

$$
A_{\|}^{r a w}=\frac{(N / Q)^{\downarrow \Uparrow}-(N / Q)^{\uparrow \Uparrow}}{(N / Q)^{\downarrow \Uparrow}+(N / Q)^{\uparrow \Uparrow}}
$$

and

$$
A_{\perp}^{\text {raw }}=\frac{(N / Q)^{\downarrow \Rightarrow}-(N / Q)^{\uparrow \Rightarrow}}{(N / Q)^{\downarrow \Rightarrow}+(N / Q)^{\uparrow \Rightarrow}}
$$

where $N$ is the detected number of electrons normalized by the total charge of the beam $Q$ in a given electron-target spin configuration. They are simply 
related to the cross section asymmetries

$$
A_{\|}=\frac{\left(\frac{d^{2} \sigma}{d \Omega d E^{\prime}}\right)^{\downarrow \Uparrow}-\left(\frac{d^{2} \sigma}{d \Omega d E^{\prime}}\right)^{\uparrow \Uparrow}}{\left(\frac{d^{2} \sigma}{d \Omega d E^{\prime}}\right)^{\downarrow \Uparrow}+\left(\frac{d^{2} \sigma}{d \Omega d E^{\prime}}\right)^{\uparrow \Uparrow}},
$$

and

$$
A_{\perp}=\frac{\left(\frac{d^{2} \sigma}{d \Omega d E^{\prime}}\right)^{\downarrow \Rightarrow}-\left(\frac{d^{2} \sigma}{d \Omega d E^{\prime}}\right)^{\uparrow \Rightarrow}}{\left(\frac{d^{2} \sigma}{d \Omega d E^{\prime}}\right)^{\downarrow \Rightarrow}+\left(\frac{d^{2} \sigma}{d \Omega d E^{\prime}}\right)^{\uparrow \Rightarrow}}
$$

by correcting for the beam polarization $P_{b}$ target polarization $P_{t}$ and the dilution factor $f$, which is the fraction of events generated by polarizable target material under consideration $\left({ }^{3} \mathrm{He}\right.$ in our experiment),

$$
\begin{aligned}
& A_{\|}=\frac{A_{\|}^{\text {raw }}}{P_{b} P_{t} f}, \\
& A_{\perp}=\frac{A_{\perp}^{\text {raw }}}{P_{b} P_{t} f} .
\end{aligned}
$$

The structure functions $g_{1}$ and $g_{2}$, eqs. (1.8)-(1.10), can now be written in terms of parallel and transverse asymmetries

$$
\begin{gathered}
g_{1}\left(x, Q^{2}\right)=\frac{F_{1}\left(x, Q^{2}\right)}{D^{\prime}}\left[A_{\|}\left(x, Q^{2}\right)+\tan \left(\frac{\theta}{2}\right) A_{\perp}\left(x, Q^{2}\right)\right] \\
g_{2}\left(x, Q^{2}\right)=\frac{F_{1}\left(x, Q^{2}\right) y}{2 D^{\prime} \sin \theta}\left[-\sin \theta A_{\|}\left(x, Q^{2}\right)+\frac{E+E^{\prime} \cos \theta}{E^{\prime}} A_{\perp}\left(x, Q^{2}\right)\right] .
\end{gathered}
$$

The various kinematic factors are defined below:

$$
\begin{aligned}
D^{\prime} & =\frac{(1-\epsilon)(2-y)}{y(1+\epsilon R)} \\
\epsilon & =\frac{1}{1+2\left(1+\left(\nu^{2} / Q^{2}\right)\right) \tan ^{2}(\theta / 2)} \\
y & =\frac{E-E^{\prime}}{E},
\end{aligned}
$$




$$
\begin{aligned}
R\left(x, Q^{2}\right) & =\frac{1+\gamma^{2}}{2 x F_{1}\left(x, Q^{2}\right)} F_{2}\left(x, Q^{2}\right)-1, \\
\gamma & =\sqrt{\frac{Q^{2}}{\nu^{2}}} .
\end{aligned}
$$

Note that the above expressions are given in terms of measurable quantities, assuming that $F_{2}\left(x, Q^{2}\right)[124]$ and $R\left(x, Q^{2}\right)$ [24, 25] are known from other experiments.

\subsection{Virtual photon-nucleon asymmetries}

The hadronic tensor $W_{\mu \nu}$ given by eq. (1.3) is also known as the virtual forward Compton amplitude, since its contraction with the virtual photon polarization four-vector $\epsilon^{\mu}$ gives the forward amplitude $\mathcal{M}$ for virtual photonproton scattering:

$$
\mathcal{M}\left(\gamma^{*} p \rightarrow \gamma^{*} p\right)=\epsilon_{\mu}^{*} \epsilon_{\nu} W^{\mu \nu}
$$

For the virtual photons, which have three polarization states, and nucleons, which have two polarizations there are four independent virtual Compton amplitudes [23, 26] (just as there are four independent structure functions),

$\mathcal{M}_{a b \rightarrow c d}$, where $a, b$ represent the incident helicities of the virtual photon and nucleon, and $c, d$ the scattered helicities, respectively. We have chosen the following independent amplitudes:

$$
\mathcal{M}_{1-\frac{1}{2} \rightarrow 1-\frac{1}{2}}, \quad \mathcal{M}_{1 \frac{1}{2} \rightarrow 1 \frac{1}{2}}, \quad \mathcal{M}_{0 \frac{1}{2} \rightarrow 0 \frac{1}{2}}, \quad \mathcal{M}_{1-\frac{1}{2} \rightarrow 0 \frac{1}{2}}
$$


Using those amplitudes, we can obtain, via the optical theorem, the total absorption cross sections of the virtual photon by the nucleon target,

$$
\begin{aligned}
\sigma_{1 / 2}=\frac{4 \pi^{2} \alpha}{K} \mathcal{M}_{1-\frac{1}{2} \rightarrow 1-\frac{1}{2}} & =\frac{4 \pi^{2} \alpha}{M K}\left[F_{1}+g_{1}-\frac{2 M x}{\nu} g_{2}\right], \\
\sigma_{3 / 2}=\frac{4 \pi^{2} \alpha}{K} \mathcal{M}_{1 \frac{1}{2} \rightarrow 1 \frac{1}{2}} & =\frac{4 \pi^{2} \alpha}{M K}\left[F_{1}-g_{1}+\frac{2 M x}{\nu} g_{2}\right], \\
\sigma_{L}=\frac{4 \pi^{2} \alpha}{K} \mathcal{M}_{0 \frac{1}{2} \rightarrow 0 \frac{1}{2}} & =\frac{4 \pi^{2} \alpha}{K}\left[\frac{F_{2}}{\nu}\left(1+\frac{\nu^{2}}{Q^{2}}\right)-\frac{F_{1}}{M}\right], \\
\sigma_{T L}=\frac{4 \pi^{2} \alpha}{K} \mathcal{M}_{1-\frac{1}{2} \rightarrow 0 \frac{1}{2}} & =\frac{4 \pi^{2} \alpha}{K} \frac{\sqrt{Q^{2}}}{M \nu}\left[g_{1}+g_{2}\right],
\end{aligned}
$$

where $K$ represents the incoming photon flux. Using Hand's convention for $K$ we have,

$$
K \equiv \frac{W^{2}-M^{2}}{2 M}=\nu-\frac{Q^{2}}{2 M}
$$

The quantities $\sigma_{1 / 2}$ and $\sigma_{3 / 2}$ are the virtual photo-absorption cross sections with the projection of the spin of the photon-nucleon system along the virtual photon axis equal to $1 / 2$ and $3 / 2$, respectively. The total transverse cross section $\sigma_{T}$ is defined as

$$
\sigma_{T} \equiv \frac{1}{2}\left(\sigma_{1 / 2}+\sigma_{3 / 2}\right)=\frac{4 \pi^{2} \alpha}{M K} F_{1}\left(x, Q^{2}\right)
$$

The cross section $\sigma_{L}$ corresponds to the longitudinally polarized photon, while $\sigma_{T L}$ comes from the interference between transverse and longitudinal amplitudes.

The virtual photon-nucleon asymmetries, $A_{1}$ and $A_{2}$, can now be defined in terms of the cross sections and related to the structure functions,

$$
A_{1}\left(x, Q^{2}\right) \equiv \frac{\sigma_{1 / 2}-\sigma_{3 / 2}}{\sigma_{1 / 2}+\sigma_{3 / 2}}=\frac{g_{1}\left(x, Q^{2}\right)-\gamma^{2} g_{2}\left(x, Q^{2}\right)}{F_{1}\left(x, Q^{2}\right)}
$$


and

$$
A_{2}\left(x, Q^{2}\right) \equiv \frac{\sigma_{T L}}{\sigma_{T}}=\frac{\gamma\left(g_{1}\left(x, Q^{2}\right)+g_{2}\left(x, Q^{2}\right)\right)}{F_{1}\left(x, Q^{2}\right)},
$$

where $\gamma$ has been defined previously, eq. (1.23). Combining the above equations with formulas $(1.17),(1.18)$ for $g_{1}$ and $g_{2}$, we can also write directly in terms of measurable longitudinal and transverse asymmetries,

$$
\begin{aligned}
& A_{1}\left(x, Q^{2}\right)=\frac{1}{D(1+\zeta \eta)} A_{\|}\left(x, Q^{2}\right)-\frac{\eta}{d(1+\zeta \eta)} A_{\perp}\left(x, Q^{2}\right), \\
& A_{2}\left(x, Q^{2}\right)=\frac{\zeta}{D(1+\zeta \eta)} A_{\|}\left(x, Q^{2}\right)+\frac{1}{d(1+\zeta \eta)} A_{\perp}\left(x, Q^{2}\right),
\end{aligned}
$$

where in addition to the previously defined kinematic factors, eq. (1.19-1.23),

$$
\begin{aligned}
D & =\frac{1-E^{\prime} \epsilon / E}{1+\epsilon R}, \\
d & =D \sqrt{\frac{2 \epsilon}{1+\epsilon}}, \\
\eta & =\frac{\epsilon \sqrt{Q^{2}}}{E-E^{\prime} \epsilon}, \\
\zeta & =\eta \frac{1+\epsilon}{2 \epsilon} .
\end{aligned}
$$

We can also interprate $R\left(x, Q^{2}\right)$ as the ratio of the longitudinal and transverse cross sections,

$$
R\left(x, Q^{2}\right) \equiv \frac{\sigma_{L}}{\sigma_{T}}=\frac{1+\gamma^{2}}{2 x F_{1}\left(x, Q^{2}\right)} F_{2}\left(x, Q^{2}\right)-1,
$$

that relates two unpolarized structure functions,

$$
F_{1}\left(x, Q^{2}\right)=\frac{1+\gamma^{2}}{2 x\left(1+R\left(x, Q^{2}\right)\right)} F_{2}\left(x, Q^{2}\right) .
$$

Note that definition (1.32) for $A_{1}$ gives the positivity (of cross sections) constraint,

$$
\left|A_{1}\left(x, Q^{2}\right)\right| \leq 1
$$


and the definition (1.33) of $A_{2}$ combined with the Schwartz inequality

$$
\|v \cdot w\|^{2} \leq\|v\|^{2}\|w\|^{2}
$$

in the form of $\sigma_{T L}^{2} \leq \sigma_{T} \sigma_{L}$ and the definition (1.40) of $R$ gives the upper bound on $A_{2}$,

$$
\left|A_{2}\left(x, Q^{2}\right)\right| \leq \sqrt{R\left(x, Q^{2}\right)} .
$$

\subsection{Models of the $\mathrm{He}^{3}$ nucleus}

In our experiment we measure the asymmetries of the polarized ${ }^{3} \mathrm{He}$. In the simplest model the nucleons in the polarized ${ }^{3} \mathrm{He}$ are in a spatially symmetric S state. Due to the Pauli principle the total wave-function must be antisymmetric, and thus the two protons have opposite spins. In this picture the spin of the ${ }^{3}$ He nucleus is carried exclusively by the neutron and the neutron asymmetries are directly proportional to those of ${ }^{3} \mathrm{He}$.

A more realistic approach [27] includes other components of the wavefunction. The dominant componenets are $S^{\prime}$ and the average of the D state components. The S' component originates in the small difference between the tensor $\mathrm{T}=0$ and $\mathrm{T}=1$ forces distorting the primary $\mathrm{S}$ wave configuration. The $\mathrm{P}$ wave contributions are suppressed since they have opposite parity.

In the $\mathrm{S}$ wave approximation the probability $P_{n}^{+}$that the neutron spin is aligned with the spin of ${ }^{3} \mathrm{He}$ equals 1 , while the probability $P_{n}^{-}$for the anti-aligned spin is 0 . For the proton $P_{p}^{+}=P_{p}^{-}=1 / 2$. Including other waves contributions we write

$$
P_{n}^{+}=1-\Delta
$$




$$
\begin{aligned}
& P_{n}^{-}=\Delta, \\
& P_{p}^{+}=\frac{1}{2}-\delta, \\
& P_{n}^{-}=\frac{1}{2}+\delta,
\end{aligned}
$$

where $\Delta=\left[P\left(S^{\prime}\right)+2 P(D)\right] / 3$ and $\delta=\left[P(D)-P\left(S^{\prime}\right)\right] / 6$ and $P\left(S^{\prime}\right), P(D)$ are the probabilities of each partial wave component. The polarization of the neutron is $p_{n}=1-2 \Delta$ and of the proton $p_{p}=-2 \delta$. The best fit to nucleon polarizations were extracted from many models [27] yielding $\Delta=0.07 \pm 0.01$ and $\delta=0.014 \pm 0.002$, and thus polarizations $p_{n}=0.86 \pm 0.02$ and $p_{p}=$ $-0.027 \pm 0.004$. This leads to the relation between asymmetries [28]

$$
A_{\|(\perp)}^{3}=f_{n} p_{n} A_{\|(\perp)}^{n}+2 f_{p} p_{p} A_{\|(\perp)}^{p},
$$

where $f_{n(p)}=F_{2}^{n(p)} /\left[\left(F_{2}^{n}+2 F_{2}^{p}\right) f_{E M C}\right]$ with the EMC effect factor $f_{E M C}[29]$, and between structure functions

$$
g_{1}^{3}{ }^{H e}(x)=p_{n} g_{1}^{n}(x)+2 p_{p} g_{1}^{p}(x)
$$

The above results neglect nucleon binding effects, Fermi motion and shadowing. The more complete calculations [28] suggest that these corrections are only important at $x>0.9$ or very low $x \sim 0$.

\subsection{Partonic interpretation}

In the parton model of Bjorken and Paschos [5], and Feynman [6] a nucleon consists of pointlike constituents, partons. Each parton carries a fraction $\xi$ of the total nucleon momentum $P$. When the nucleon is viewed from 
the infinite momentum frame [30], one argues, ignoring the transverse motion of constituents, that the interaction between partons is time dilated. If the probing momentum (and energy) of the virtual photon is large, i.e. in the Bjorken limit, the virtual photon-parton interaction is short in comparison with parton-parton interactions. The inclusive scattering may be considered as incoherent elastic scattering off effectively free pointlike constituents. For the scattered parton of mass $m_{q}$ we write

$$
(\xi P+q)^{2}=m_{q}^{2}
$$

In the limit of large momentum transfer compared to the parton and nucleon masses,

$$
\xi=\frac{Q^{2}}{2 M \nu} \equiv x
$$

The virtual photon with $x$ defined by kinematics only scatters off the parton with momentum fraction $\xi=x$. Or, equivalently, the Bjorken variable $x$ is the fraction of the nucleon momentum carried by the struck quark.

The remarkable feature of this model is Bjorken scaling. We treat the parton as a spin 1/2 Dirac particle, the quark, and calculate structure functions for scattering off such a particle. We then sum incoherently over contributions from all single quarks weighted by the probabilities $q^{\uparrow(\downarrow)}(x)\left(\bar{q}^{\uparrow(\downarrow)}(x)\right)$ of finding a quark (antiquark) with a spin direction the same $(\uparrow)$ or opposite $(\downarrow)$ to the spin of the nucleon, carrying the fraction $x$ of the nucleon momentum. Taking the Bjorken limit

$$
Q^{2} \rightarrow \infty, \nu \rightarrow \infty \text { with } \quad x=\frac{Q^{2}}{2 M \nu}=\text { const }
$$


of the structure functions, one finds the Bjorken scaling:

$$
f\left(x, Q^{2}\right) \stackrel{B j}{\longrightarrow} f(x)
$$

where $f$ stands for $F_{1}, F_{2}, g_{1}$ or $g_{2}$. The structure functions only depend on one variable $x$. This $x$ dependence is defined by the quark probability distributions,

$$
\begin{aligned}
q(x) & \equiv\left[q^{\uparrow}(x)+\bar{q}^{\uparrow}(x)\right]+\left[q^{\downarrow}(x)+\bar{q}^{\downarrow}(x)\right], \\
\Delta q(x) & \equiv\left[q^{\uparrow}(x)+\bar{q}^{\uparrow}(x)\right]-\left[q^{\downarrow}(x)+\bar{q}^{\downarrow}(x)\right] .
\end{aligned}
$$

Namely,

$$
F_{1}(x)=\frac{1}{2} \sum_{f} e_{f}^{2} q_{f}(x),
$$

and

$$
g_{1}(x)=\frac{1}{2} \sum_{f} e_{f}^{2} \Delta q_{f}(x),
$$

where the sum is over all quark flavors $f$, and $e_{f}$ denotes the quark charge.

Furthermore, in the Bjorken limit, the Callan-Gross [31] relation

$$
F_{2}(x)=2 x F_{1}(x)
$$

holds. When substituted into the definition of $R\left(x, Q^{2}\right)$ in eq. (1.40) taken in the Bjorken limit, $(\gamma \stackrel{B j}{\longrightarrow} 0$, eq. $(1.23))$, it gives

$$
R\left(x, Q^{2}\right)=0
$$

that is

$$
\sigma_{L}\left(x, Q^{2}\right)=0
$$


as seen from eq. (1.28). Also, see eq. (1.29),

$$
\sigma_{T L}\left(x, Q^{2}\right)=0 .
$$

The last equation implies that from the definition (1.33)

$$
A_{2}\left(x, Q^{2}\right)=0 .
$$

It is instructive to look at the Bjorken limit of the virtual photon asymmetry $A_{1}$. From the definition (1.32) and the partonic expressions on $g_{1}$ and $F_{1}$ we get

$$
A_{1}\left(x, Q^{2}\right)=\frac{g_{1}\left(x, Q^{2}\right)}{F_{1}\left(x, Q^{2}\right)}=\frac{\sum_{f} e_{f}^{2} \Delta q_{f}(x)}{\sum_{f} e_{f}^{2} q_{f}(x)} .
$$

The last equality can also be inferred from the following considerations [26]: Let the nucleon $P^{\uparrow}$ with the projection $J_{z}=+\frac{1}{2}$ collide with a transversely polarized photon $\gamma^{\uparrow(\downarrow)}$ with $J_{z}=+1(\uparrow)$ or $-1(\downarrow)$. The total spin angular momentum is $J_{z}=\frac{3}{2}$ or $\frac{1}{2}$ :

$$
\begin{array}{ll}
\gamma^{\uparrow}+P^{\uparrow} & \rightarrow \sigma_{3 / 2}, \\
\gamma^{\downarrow}+P^{\uparrow} & \rightarrow \sigma_{1 / 2} .
\end{array}
$$

The photon-quark interaction is collinear (neglecting the transverse motion of parton) so that the projection of orbital angular momentum is zero. Hence, from angular momentum conservation, the transverse photon will flip the spin of the quark with which it interacts,

$$
\begin{aligned}
& \gamma^{\uparrow}+q^{\downarrow} \rightarrow q^{\uparrow}, \\
& \gamma^{\downarrow}+q^{\uparrow} \rightarrow q^{\downarrow},
\end{aligned}
$$


but $\gamma^{\uparrow} q^{\uparrow}$ and $\gamma^{\downarrow} q^{\downarrow}$ are suppressed. So we see that

$$
\begin{aligned}
& \sigma_{3 / 2} \sim \gamma^{\uparrow} P^{\uparrow} \sim \sum_{f} e_{f}^{2} q_{f}^{\downarrow}, \\
& \sigma_{1 / 2} \sim \gamma^{\downarrow} P^{\uparrow} \sim \sum_{f} e_{f}^{2} q_{f}^{\uparrow},
\end{aligned}
$$

leading via definition (1.32) of $A_{1}=\frac{\sigma_{1 / 2}-\sigma_{3 / 2}}{\sigma_{1 / 2}+\sigma_{3 / 2}}$ to equation (1.61).

As an example [32] of $A_{1}(x)$ asymmetry let us consider the $\mathrm{SU}(6)$ symmetric constituent quark model. In this model the proton consists of three valence quarks. The spin-flavor part of its wave-function can be written as

$$
P^{\uparrow}=\frac{1}{\sqrt{6}}(2 \uparrow \uparrow \downarrow-\uparrow \downarrow \uparrow-\downarrow \uparrow \uparrow) \mathrm{uud}
$$

in the uds basis. The momentum part of the wave-function is totally symmetric, implying that the $x$ dependence of partonic distributions, denoted by $p(x)$, is the same. The probability distribution for finding a down quark with the spin parallel to the proton spin can be obtained from the spin-flavor composition of the proton in eq. (1.68) as $d^{\uparrow}(x)=\frac{1}{6}\left[(-1)^{2}+(-1)^{2}\right] p(x)=\frac{1}{3} p(x)$, where $p(x)$ is normalized to $1=\int_{0}^{1} d x p(x)$, and the probability of finding a quark with any flavor and spin is normalized to the number of quarks (to 3 in our case). Similarly, we get

$$
\begin{aligned}
u^{\uparrow}(x) & =\frac{5}{3} p(x), \\
u^{\downarrow}(x) & =\frac{1}{3} p(x), \\
d^{\uparrow}(x) & =\frac{1}{3} p(x), \\
d^{\downarrow}(x) & =\frac{2}{3} p(x) .
\end{aligned}
$$


Substituting these distributions into (1.61) we find

$$
A_{1}^{p}(x)=\frac{\frac{4}{9}\left(u^{\uparrow}(x)-u^{\downarrow}(x)\right)+\frac{1}{9}\left(d^{\uparrow}(x)-d^{\downarrow}(x)\right)}{\frac{4}{9}\left(u^{\uparrow}(x)+u^{\downarrow}(x)\right)+\frac{1}{9}\left(d^{\uparrow}(x)+d^{\downarrow}(x)\right)}=\frac{5}{9}
$$

for the proton. The neutron asymmetry is related to the proton asymmetry via isospin exchange $u \leftrightarrow d$, so

$$
A_{1}^{n}(x)=0
$$

In a more realistic model with $\mathrm{SU}(6)$ breaking admixtures [33] one obtains a nontrivial $x$ dependence of the nucleon asymmetries [34].

The total spin $\Delta \Sigma$ carried by the quarks can be defined as the sum of the net spin of all contributing flavors:

$$
\Delta \Sigma=\sum_{f} \int_{0}^{1} d x \Delta q_{f}(x) \equiv \sum_{f} \Delta q_{f} .
$$

In the three flavor model

$$
\Delta \Sigma=\Delta u+\Delta d+\Delta s .
$$

For the nucleon, with the values of $\Delta q_{f}(x)$ from the SU(6) model given in eqs. $(1.69-1.72)$ we obtain

$$
\Delta \Sigma=1
$$

The spin of the nucleon is entirely carried by constituent quarks.

\subsection{QCD improved parton model}

The quark-parton model considered in the previous section can be for-

mulated more rigorously on the basis of QCD. The main modifications come 
from the radiative corrections. Quarks within a nucleon can emit gluons, which in turn can produce $q \bar{q}$ pairs. These processes lead to scaling violations logarithmic in $Q^{2}$. The structure functions and the partonic distributions are no longer functions of just the $x$ variable. They acquire additional $Q^{2}$ dependence.

The $g_{1}$ structure function, for example, can still (in leading order) be written in the partonic form of eq. (1.55)

$$
g_{1}\left(x, Q^{2}\right)=\frac{1}{2} \sum_{f} e_{f}^{2} \Delta q_{f}\left(x, Q^{2}\right) .
$$

That the structure function, directly related to the cross section, can be written in terms of partonic distributions and some coefficients is a manifestation of a more general property, the factorization of DIS cross sections (see section 4.1, page 177 ).

The $Q^{2}$ dependence of the partonic distributions is described by the Altarelli-Parisi equations (cf. section 4.2, page 179),

$$
\begin{aligned}
Q^{2} \frac{d}{d Q^{2}} \Delta q_{f}\left(x, Q^{2}\right) & =\frac{\alpha_{s}\left(Q^{2}\right)}{2 \pi}\left[P_{q q} \otimes \Delta q_{f}+P_{q G} \otimes \Delta G\right] \\
Q^{2} \frac{d}{d Q^{2}} \Delta G\left(x, Q^{2}\right) & =\frac{\alpha_{s}\left(Q^{2}\right)}{2 \pi}\left[P_{G q} \otimes \sum_{f}^{2 N_{f}} \Delta q_{f}+P_{G G} \otimes \Delta G\right]
\end{aligned}
$$

The so called splitting functions $P_{i j}(x)$ are the hard scattering cross sections of the radiative processes. The convolution $\otimes$ is defined as

$$
P \otimes \Delta q=\int_{x}^{1} \frac{d y}{y} P\left(\frac{x}{y}\right) \Delta q\left(y, Q^{2}\right)
$$

The total spin $\Delta q=\int_{0}^{1} d x \Delta q(x)$ carried by the quark can be defined in the 
operator language as the matrix element of the axial current,

$$
\Delta q S^{\mu}=\left\langle P S\left|\bar{q} \gamma^{\mu} \gamma_{5} q\right| P S\right\rangle=\left\langle P S\left|\bar{q}_{R} \gamma^{\mu} q_{R}-\bar{q}_{L} \gamma^{\mu} q_{L}\right| P S\right\rangle
$$

where $q_{R, L} \equiv \frac{1}{2}\left(1 \pm \gamma_{5}\right) q$. With this definition we can decompose the $\mathrm{SU}(3)_{\text {flavor }}$ axial-vector currents $j_{a}^{\mu}$, defined as

$$
j_{a}^{\mu}=\bar{\psi} \gamma^{\mu} \gamma_{5} \tau_{a} \psi
$$

Here $\psi$ is the flavor triplet,

$$
\psi=\left(\begin{array}{c}
u \\
d \\
s
\end{array}\right)
$$

and $\tau_{a}$ are the $\mathrm{SU}(3)$ generators. With the flavor indices shown explicitly the currents read,

$$
j_{a}^{\mu}=\sum_{i, j=1}^{3} \bar{\psi}_{i} \gamma^{\mu} \gamma_{5}\left(\tau_{a}\right)_{i j} \psi_{j}
$$

Similarly to eq. (1.82) let us introduce axial charges $\Delta q_{a}$ as appropriate matrix elements of the $j_{a}^{\mu}$ currents:

$$
\Delta q_{a} S^{\mu}=\left\langle P S\left|j_{a}^{\mu}\right| P S\right\rangle
$$

Of special interest to DIS are the $a=0, \mathrm{SU}(3)_{\text {flavor }}$ singlet and the $a=3,8$ non-singlet axial-vector currents from the octet. With our choice of the normalization of the $\tau_{a}$ generators,

$$
\tau_{0}=\operatorname{Id}(3), \quad \tau_{3}=\left(\begin{array}{rrr}
1 & 0 & 0 \\
0 & -1 & 0 \\
0 & 0 & 0
\end{array}\right), \quad \tau_{8}=\left(\begin{array}{rrr}
1 & 0 & 0 \\
0 & 1 & 0 \\
0 & 0 & -2
\end{array}\right)
$$


and with the help of eqs. (1.82-1.86) we can rewrite the corresponding charges in a more familiar form,

$$
\begin{aligned}
\Delta q_{0} & =\Delta u+\Delta d+\Delta s \equiv \Delta \Sigma \\
\Delta q_{3} & =\Delta u-\Delta d \\
\Delta q_{8} & =\Delta u+\Delta d-2 \Delta s .
\end{aligned}
$$

The octet currents $j_{3}^{\mu}$ and $j_{8}^{\mu}$ are conserved. Thus the associated charges $\Delta q_{3}$ and $\Delta q_{8}$ are $Q^{2}$ independent. The singlet axial current is conserved in the leading order only. In general it acquires an anomalous contribution. The classical QCD lagrangian for massless quarks is invariant under the global $\mathrm{U}(1)$ axial (chiral) transformation

$$
\psi \rightarrow \psi^{\prime}=e^{-i \theta \gamma_{5}} \psi
$$

The singlet axial current is a Noether current arising from this symmetry. Thus on the classical level it is conserved and

$$
\partial^{\mu} j_{\mu}^{0}=0
$$

However, in the full quantum theory the axial U(1) transformation is not a symmetry. The current divergence has an anomaly [35], which arises from triangular diagrams of quantum corrections,

$$
\partial^{\mu} j_{\mu}^{0}=\frac{N_{f} \alpha_{s}}{8 \pi} F_{\mu \nu}^{a} \widetilde{F}^{a \mu \nu}
$$

where $\widetilde{F}^{a}$ is a dual to $F^{a}$, i.e. $\widetilde{F}^{a \mu \nu}=\epsilon^{\mu \nu \rho \sigma} F_{\rho \sigma}^{a}$, and $N_{f}$ is the number of flavors. In the path integral formulation of the quantum theory one sees that 
the path integral measure is not invariant under the $\mathrm{U}(1)$ transformation although the lagrangian is [36].

One can still construct a divergence-free axial current by noting that the anomalous contribution on the right hand side of eq. (1.93) can be written as the divergence of an axial gluon current, $K^{\mu}[37]$,

$$
\partial_{\mu} K^{\mu}=\frac{1}{4} F_{\mu \nu}^{a} \widetilde{F}^{a \mu \nu}
$$

We can now redefine $j_{\mu}^{0}$,

$$
\widetilde{j}_{\mu}^{0}=j_{\mu}^{0}-N_{f} \frac{\alpha_{s}}{2 \pi} K_{\mu},
$$

so that the new current is conserved,

$$
\partial^{\mu} \widetilde{j}_{\mu}^{0}=0
$$

Note that the axial gluon current is not gauge invariant. In the lightcone gauge $A^{+}=0$, its expectation value in the polarized nucleon is identified with the gluon spin contribution [38],

$$
\Delta G S^{\mu}=-\left\langle P S\left|K^{\mu}\right| P S\right\rangle .
$$

The conserved charge, $\Delta \widetilde{q_{0}}$, differs from the old charge by the anomalous gluon contribution,

$$
\Delta \widetilde{q_{0}}=\Delta q_{0}+N_{f} \frac{\alpha_{s}}{2 \pi} \Delta G
$$

One can argue that $\Delta \widetilde{q_{0}}$ should be identified with the total quark helicity,

$$
\Delta \widetilde{\Sigma}=\Delta \Sigma+N_{f} \frac{\alpha_{s}}{2 \pi} \Delta G
$$


This ambiguity is a manifestation of factorization scheme dependence, which will be treated in some detail in section 4.6 (page 197). We note that $\alpha_{s}\left(Q^{2}\right) \Delta G\left(Q^{2}\right) \approx$ constant, so the ambiguity persists, even in the large $Q^{2}$ limit.

There is one more source of the scaling violation of structure functions in addition to the $Q^{2}$ evolution. It is referred to as higher twist corrections and gives contributions which are suppressed by powers of $Q^{2}$ with respect to the leading twist. They could be interpreted [39] as coming from the diagrams involving interaction between different quarks or from quark-gluon correlations. These corrections are expected to be important at low values of $W^{2}$ for high $x$ and low values of $Q^{2}$ for low $x$. For example, for the $F_{2}$ structure function we can write

$$
F_{2}\left(x, Q^{2}\right)=F_{2}^{l t}\left(x, Q^{2}\right)\left[1+\sum_{m=1} \frac{D_{m}}{\left(Q^{2}(1-x)\right)^{m}}\right],
$$

where $F^{l t}$ denotes the leading twist part. The unpolarized data from SLAC $[24,25]$ show sizeble higher twist contributions at high $x$. Thus we conclude that higher twist contributions to the polarized structure functions are important at low $W^{2}$.

\subsection{Sum rules}

\subsubsection{Helicity sum rule}

Since the nucleon is a spin $1 / 2$ particle, $|P S\rangle$ is an eigenstate of the Pauli-Lubanski vector operator $W_{\mu}=\epsilon_{\mu \nu \rho \sigma} J^{\nu \rho} P^{\sigma} /(2 M)$ projected onto the 
polarization $S^{\mu}$,

$$
S^{\mu} W_{\mu}|P S\rangle=\frac{1}{2}|P S\rangle
$$

or equivalently,

$$
\frac{1}{2}=\left\langle P S\left|S^{\mu} W_{\mu}\right| P S\right\rangle
$$

For $\vec{S}$ along the direction of $\vec{P}$ (chosen to be the $\mathrm{z}$ axis) the nucleon is in the $1 / 2$ helicity eigenstate, and

$$
S^{\mu} W_{\mu}=J^{z}
$$

so one can construct a helicity sum rule,

$$
\frac{1}{2}=\left\langle P S\left|J^{z}\right| P S\right\rangle
$$

To be more specific we need to consider the transformation properties of the QCD lagrangian under spatial rotations and construct the generators of rotations, i.e. the angular momentum operator [40]. In a shorthand notation the angular momentum operator can be written as

$$
\vec{J}=\int d^{3} x\left[\frac{1}{2} \bar{\psi} \vec{\gamma} \gamma_{5} \psi+\psi^{\dagger}(\vec{x} \times(-i \vec{\nabla})) \psi+\vec{E} \times \vec{A}+E_{i}(\vec{x} \times \vec{\nabla}) A_{i}\right] .
$$

The physical interpretation of the above equation is obvious. The first term is the quark spin contribution to the nucleon helicity, the second is the quark orbital angular momentum, the third is the gluon spin and the last term is the gluon orbital momentum. Taking the expectation value of $J^{z}$ as in eq. (1.104) and choosing the light cone gauge $\left(A^{+}=0\right)$ in the infinite momentum frame, we obtain the sum rule

$$
\frac{1}{2}=\frac{1}{2} \Delta \Sigma\left(Q^{2}\right)+L_{q}\left(Q^{2}\right)+\Delta G\left(Q^{2}\right)+L_{G}\left(Q^{2}\right)
$$


for the nucleon spin. It is evident from the decomposition (1.105) that $L_{q}$, $\Delta G$ and $L_{G}$ are gauge dependent. They are also scale dependent. One can write the evolution equations for those quantities [41, 42] and find the asymptotic, $Q^{2} \rightarrow \infty$, behavior

$$
\begin{aligned}
\Delta \Sigma & \rightarrow \text { const. } \\
L_{q} & \rightarrow \text { const. } \\
\Delta G & \rightarrow \lambda \log \left(Q^{2}\right)+\text { const. } \\
L_{G} & \rightarrow-\lambda \log \left(Q^{2}\right)+\text { const. }
\end{aligned}
$$

The asymptotic increase of gluon helicity is canceled by the gluon orbital contribution. Recently, [43], an explicitly gauge invariant decomposition of the angular momentum operator

$$
\vec{J}=\int d^{3} x\left[\frac{1}{2} \bar{\psi} \vec{\gamma} \gamma_{5} \psi+\psi^{\dagger}(\vec{x} \times(-i \vec{D})) \psi+\vec{x} \times(\vec{E} \times \vec{B})\right],
$$

has been proposed, leading to a gauge invariant spin sum rule,

$$
\frac{1}{2}=\left(\frac{1}{2} \Delta \Sigma\left(Q^{2}\right)+L_{q}^{\prime}\left(Q^{2}\right)\right)+J_{G}\left(Q^{2}\right)
$$

or

$$
\frac{1}{2}=J_{q}\left(Q^{2}\right)+J_{G}\left(Q^{2}\right)
$$

There are two additional interesting features of the above sum rule [42, 44]. Study of the $Q^{2}$ dependence of $J_{q}$ and $J_{G}$ reveals that the anomalous dimension matrix for these two operators has a zero eigenvalue. This implies that in the large $Q^{2}$ limit, where all other eigenvectors of the anomalous dimension 
matrix have evolved to zero, the ratio of quark and gluon contributions goes to a definite limit, independent of hadronic target. There is an analogous result for the momentum sum rule [45] which gives the same fractions,

$$
\begin{aligned}
J_{q}\left(Q^{2} \rightarrow \infty\right) & \rightarrow \frac{1}{2} \frac{3 N_{f}}{16+3 N_{f}}, \\
J_{G}\left(Q^{2} \rightarrow \infty\right) & \rightarrow \frac{1}{2} \frac{16}{16+3 N_{f}} .
\end{aligned}
$$

Study of the $J_{q}$ in a factorization scheme in which $\Delta \Sigma$ has an explicit triangle anomaly added to it, $\Delta \Sigma+N_{f} \frac{\alpha}{2 \pi} \Delta G=\Delta \widetilde{\Sigma}$, shows that at the same time the quark orbital angular momentum has a compensating gluonic contamination,

$$
\begin{aligned}
\widetilde{J}_{q} & =\frac{1}{2} \Delta \widetilde{\Sigma}+\left(L_{q}-N_{f} \frac{\alpha}{4 \pi} \Delta G\right) \\
& =\frac{1}{2} \Delta \Sigma+L_{q}=J_{q} .
\end{aligned}
$$

Thus the net effect of the factorization scheme dependence is to shift a contribution between the quark spin and orbital angular momentum, leaving $J_{q}$ unchanged.

\subsubsection{Bjorken sum rule}

The Bjorken sum rule [46] follows from isospin symmetry and the short distance analysis of QCD. It tests QCD at a very fundamental level. Originally derived in the context of current algebra before the advent of QCD, it connects the low $Q^{2}$ physics of the neutron beta decay with the high $Q^{2}$ physics of deep inelastic scattering. In the scaling limit it has the form,

$$
\int_{0}^{1} d x\left(g_{1}^{p}(x)-g_{1}^{n}(x)\right)=\frac{1}{6} g_{A},
$$


where $g_{A}$ is the axial coupling constant of the neutron beta decay. Let us briefly sketch the idea underlying the derivation [47]. The neutron beta decay is governed by the matrix element of the isovector axial current,

$$
\left\langle p\left|\bar{\psi} T^{+} \gamma^{\mu} \gamma_{5} \psi\right| n\right\rangle=S^{\mu} g_{A}
$$

where $T^{+}$is the raising operator of the $\mathrm{SU}(2)$ isospin algebra. Substituting $|n\rangle=T^{-}|p\rangle$ and using $T^{+}|p\rangle=0$ and $\left[T^{+}, T^{-}\right]=T^{3}$, we write,

$$
S^{\mu} g_{A}=\left\langle p\left|\bar{\psi} T^{3} \gamma^{\mu} \gamma_{5} \psi\right| p\right\rangle
$$

Now, recalling

$$
T^{3}\left(\begin{array}{l}
u \\
d \\
s
\end{array}\right)=\left(\begin{array}{r}
u \\
-d \\
0
\end{array}\right),
$$

we connect the last equation with the helicities of the $u$ and $d$ quarks,

$$
S^{\mu} g_{A}=\left\langle p\left|\bar{\psi} T^{3} \gamma^{\mu} \gamma_{5} \psi\right| p\right\rangle=\left\langle p\left|\bar{u} \gamma^{\mu} \gamma_{5} u-\bar{d} \gamma^{\mu} \gamma_{5} d\right| p\right\rangle=S^{\mu}(\Delta u-\Delta d),
$$

that is

$$
\Delta u-\Delta d \equiv \Delta q_{3}=g_{A}=1.2601 \pm 0.0025
$$

To proceed let us express the integrals of $g_{1}^{p}(x)$ and $g_{1}^{n}(x)$, see eq. (1.55), in terms of the axial charges (1.88-1.90),

$$
\begin{aligned}
\Gamma^{p} & \equiv \int_{0}^{1} d x g_{1}^{p}(x)=\frac{1}{12} \Delta q_{3}+\frac{1}{36} \Delta q_{8}+\frac{1}{9} \Delta q_{0} \\
\Gamma^{n} \equiv \int_{0}^{1} d x g_{1}^{n}(x) & =-\frac{1}{12} \Delta q_{3}+\frac{1}{36} \Delta q_{8}+\frac{1}{9} \Delta q_{0}
\end{aligned}
$$

and arrive at the final result,

$$
\int_{0}^{1} d x\left(g_{1}^{p}(x)-g_{1}^{n}(x)\right) \equiv \frac{1}{6} \Delta q_{3}=\frac{1}{6} g_{A} .
$$


The right hand side of the last equation has been established from the neutron beta decay in the limit of $Q^{2} \rightarrow 0$. Fortunately, the left hand side is proportional to $\Delta q_{3}$, which is conserved, and thus can be established at any value of $Q^{2}$, e.g. on the scale of DIS experiments. As mentioned earlier, the above formulation of the Bjorken sum rule is valid in the scaling limit. The coefficient $\frac{1}{6}$, called the Wilson coefficient, comes from the factorization (see section 4.1, page 177) of the scattering cross section (and thus structure functions) into soft partonic distributions and hard scattering cross sections (Wilson coefficients). At finite $Q^{2}$ the perturbatively calculated coefficient is

expressed as a series in the strong coupling constant [48]. This gives the $Q^{2}$ dependence to the Bjorken sum rule,

$$
\Gamma^{p}-\Gamma^{n}=\frac{1}{6} g_{A}\left[1-\frac{\alpha_{s}\left(Q^{2}\right)}{\pi}+C_{2}\left(\frac{\alpha_{s}\left(Q^{2}\right)}{\pi}\right)^{2}+C_{3}\left(\frac{\alpha_{s}\left(Q^{2}\right)}{\pi}\right)^{3}\right] \text {, }
$$

where $C_{2}=-3.5833$ and $C_{3}=-20.2153$ in the three flavor theory. The corresponding expansion for $\Gamma^{p} \operatorname{reads}[49]$ up to $O\left(\alpha_{s}^{2}\right)$,

$$
\Gamma^{p}\left(Q^{2}\right)=\frac{1}{6}\left[\left(\frac{1}{2} \Delta q_{3}+\frac{1}{6} \Delta q_{8}\right)\left(1-\frac{\alpha_{s}}{\pi}\right)+\frac{2}{3} \Delta q_{0}\left(1-\frac{\alpha_{s}}{\pi} \frac{33-8 N_{f}}{33-2 N_{f}}\right)\right] .
$$

\subsubsection{Ellis-Jaffe sum rule}

In addition to the value of $\Delta q_{3}$ and with the assumption of the $\mathrm{SU}(3)_{\text {flavor }}$ symmetry for the octet baryon currents, one obtains $\Delta q_{8}$ from the hyperon beta decays [50],

$$
\Delta q_{8}=0.579 \pm 0.025 .
$$


In a simple model the nucleon consists of $u$ and $d$ valence quarks, and perturbative QCD generates quark-antiquark pairs, perhaps not highly correlated with the spin of the nucleon. Furthermore, the greater mass of the strange quark suggests that the strange sea is suppressed in comparison with the light $q \bar{q}$ pairs. Thus it has been conjectured [51] that $\Delta s$ contribution can be neglected, which implies

$$
\Delta s=0 \Rightarrow \Delta q_{0}=\Delta q_{8}
$$

so that $\Gamma^{p}$ and $\Gamma^{n}$ can be computed. At $Q^{2}=5 \mathrm{GeV}^{2}$ the values are

$$
\begin{aligned}
& \Gamma^{p} \equiv \int_{0}^{1} d x g_{1}^{p}(x)=0.172 \pm 0.003 \\
& \Gamma^{n} \equiv \int_{0}^{1} d x g_{1}^{n}(x)=-0.020 \pm 0.003 .
\end{aligned}
$$

The results for the integrals of $g_{1}^{p}(x)$ and $g_{1}^{n}(x)$ with the assumption of eq. (1.125) are known as Ellis-Jaffe sum rules.

We note that from the values of $\Delta q_{3}$ and $\Delta q_{8}$ from beta decays and measuring $\Gamma^{p}$ or $\Gamma^{n}$ one can extract $\Delta \Sigma\left(=\Delta q_{0}\right)$ or, equivalently, the total spin carried by different flavors, $\Delta u, \Delta d$ and $\Delta s$.

\subsubsection{Sum rules for $g_{2}$}

The transverse spin structure function $g_{2}$ is a higher twist structure function that has been recently measured $[53,54]$. The operator product expansion (OPE) analysis reveals that $g_{2}$ consists of two pieces: one entirely twist two part $[55] g_{2}^{\text {ww }}$ and the true twist three part $\bar{g}_{2}$, with

$$
g_{2}\left(x, Q^{2}\right)=g_{2}^{w w}\left(x, Q^{2}\right)+\bar{g}_{2}\left(x, Q^{2}\right)
$$


The function $g_{2}^{w w}$ is related to thestructure function $g_{1}$ by

$$
g_{2}^{u w}\left(x, Q^{2}\right)=-g_{1}\left(x, Q^{2}\right)+\int_{x}^{1} \frac{d y}{y} g_{1}\left(y, Q^{2}\right) .
$$

The part $\bar{g}_{2}$ measures quark-gluon correlations in the nucleon. Its moments are related to the local operator products such as

$$
\int_{0}^{1} d x x^{2} \bar{g}_{2}\left(x, Q^{2}\right) \propto\left\langle P S\left|\bar{\psi} \widetilde{G}_{\sigma\left(\mu_{1}\right.} \gamma_{\left.\mu_{2}\right)} \psi\right| P S\right\rangle .
$$

The interesting Burkhardt-Cottingham [56] sum rule for $g_{2}$ is a consequence of the rotational invariance and some assumptions about the Regge behavior of the Compton amplitude,

$$
\int_{0}^{1} d x g_{2}\left(x, Q^{2}\right)=0
$$

Its test could provide some insight into the applicability of Regge theory to DIS. Note that Wandzura-Wilczek piece of $g_{2}$ obeys the Burkhardt-Cottingham sum rule,

$$
\int_{0}^{1} d x g_{2}^{w w}\left(x, Q^{2}\right)=0
$$

provided $g_{1}$ is sufficiently well behaved when approaching $x=0$ to allow the exchange of the $\mathrm{x}$ and $\mathrm{y}$ integrations.

The OPE also provides us with the moment sum rules for $g_{1}$ and $g_{2}$

$$
\begin{array}{lll}
\int_{0}^{1} d x x^{n} g_{1}\left(x, Q^{2}\right) & =\frac{1}{4} a_{n} & n=0,2,4, \ldots \\
\int_{0}^{1} d x x^{n} g_{1}\left(x, Q^{2}\right) & =\frac{1}{4} \frac{n}{n+1}\left(d_{n}-a_{n}\right) & n=2,4, \ldots
\end{array}
$$

There is no information about the $n=0$ moment of $g_{2}$ i.e. the OPE does not say anything about the Burkhardt-Cottingham sum rule. The knowledge of 
all the $\left\{a_{n}, n=0,2,4, \ldots\right\}$ and all the $\left\{d_{n}, n=2,4, \ldots\right\}$ does not completely determine $g_{2}\left(x, Q^{2}\right)$ because the lowest moment is unknown.

\subsection{Traditional evaluation of sum rules from measurements}

The measurements of the structure functions only cover a limited range in $x$ and, due to spectrometer kinematics, each point $x_{i}$ is obtained at a different (averaged over the $x$ bin) scale $Q_{i}^{2}$. To evaluate any of the sum rules, one needs to evolve the data to a single value of $Q^{2}$ and then extrapolate the result to $x=0$ and $x=1$.

\subsubsection{Evolution in $Q^{2}$}

The value of $Q^{2}$ to which all the measurements are evolved is arbitrary. However, each experiment has a characteristic scale, namely, the statistically weighted average $\left\langle Q^{2}\right\rangle$. The evolved values are quoted at this average $\left\langle Q^{2}\right\rangle$ of the experiment.

The traditional evolution technique is based on the assumption that the ratio $g_{1}\left(x, Q^{2}\right) / F_{1}\left(x, Q^{2}\right)$, which is approximately equal to $A_{1}\left(x, Q^{2}\right)$, scales, i.e. is constant in $Q^{2}$. Here the function $F_{1}\left(x, Q^{2}\right)$ is considered to be known. For the present kinematic range in $Q^{2}$ and the precision of the data, this assumption is compatible with experimental evidence. The common wisdom for $A_{1}$ scaling is based on the following 'hand waving' argument. We know from perturbative QCD (pQCD) that both structure functions $g_{1}$ and $F_{1}$ 
vary with $Q^{2}$. If these variations are 'somewhat similar' (if they factor, one should say) then when taking the ratio $g_{1} / F_{1}$ the variations cancel out at least partially, so the $g_{1} / F_{1}$ varies less strongly with $Q^{2}$ than $g_{1}$ and $F_{1}$. The 'somewhat similar evolution' phrase is sometimes misleadingly quantified in terms of the identity of the splitting functions $P_{q q}$ for polarized and unpolarized evolutions in the leading order. In the $x$ region where the valence quarks dominate, it is argued, the variation of $g_{1}$ and $F_{1}$ is the same (meaning only that the splitting functions are the same). However, because the AltarelliParisi equations are integro-differential equations, see eq. (1.79), (1.80), the $x$ dependence of the distributions matters, and 'the same splitting functions' argument is valid only if the $x$ shapes of both structure functions are the same, i.e. $F_{1}\left(x, Q_{i}^{2}\right)=$ const. $* g_{1}\left(x, Q_{i}^{2}\right)$ at the initial $Q_{i}^{2}$.

For the evolution of the precise data one has to refer directly to the pQCD formalism, as in Chapter 4 (page 176).

\subsubsection{The low $x$ extrapolation}

The low $x$ extrapolation has traditionally been performed with the assumption of Regge behavior of $g_{1}\left(x, Q^{2}\right)$. At a constant $Q^{2}$ and $x \approx 0$, the $g_{1}\left(x, Q^{2}\right)$ converges as

$$
g_{1}\left(x, Q^{2}\right) \underset{x \rightarrow 0}{\sim} x^{-\alpha},
$$

where the leading intercept $\alpha$, estimated to lay between -0.5 and 0.0 , is believed to come from the $a_{1}$ meson trajectory $[37,57]$. The experiments have assumed that at their $Q^{2}$ the few lowest $x$ measurements are in a kinematic 
region of Regge behavior and have extrapolated data to $x=0$ with $\alpha=0.0$. The variation in $\alpha$ has been used to estimate the extrapolation error. There is no theoretical guidance as tp the values of $x$ and $Q^{2}$ at which the Regge behavior sets in. Furthermore there are also other possibilities for the low $x$ behavior, even within the Regge theory. We come back to that subject in more detail in section 4.3 .2 (page 187).

\subsubsection{The high $x$ extrapolation}

The high $x$ behavior used for extrapolations seems to be much less controversial, perhaps mainly due to the fact that the structure functions decrease rapidly with $x$ and the extrapolation contribution to the integral is typically small. From QCD arguments (see section 4.3.1, page 182 for details) formulated in terms of the counting rules [58], we expect the fall off

$$
g_{1}(x) \underset{x \rightarrow 1}{\sim}(1-x)^{3},
$$

or stronger, due to the QCD evolution. The same considerations lead to the conclusion that the quark that carry almost all of the nucleon momentum also carry its spin, the opposite helicity being suppressed by another $(1-x)^{2}$. Thus at $x \sim 1 F_{1}$ is dominated by the quark distribution $q(x)=q^{\uparrow}(x)$, which is also dominant in $g_{1}, \Delta q(x)=q^{\uparrow}(x)$, and

$$
A_{1} \sim \frac{\Delta q(x)}{q(x)} \underset{x \rightarrow 1}{\sim} \frac{q^{\uparrow}(x)}{q^{\uparrow}(x)}=1 .
$$

Both extrapolations, eq. (1.135) and (1.136), have been used and have given consistent results. 


\subsection{Summary of polarized DIS experiments}

As mentioned in section 1.1, the first polarized DIS experiment, SLAC E80 [14] was initiated by the Yale-SLAC group and performed in 1976. A beam of polarized electrons was scattered off a polarized proton target, and the first measurement of the proton asymmetry $A_{1}^{p}$ was made. An atomic beam source was used for the production of polarized electrons. A beam of ${ }^{6} \mathrm{Li}$ atoms was polarized by filtering through a Stern-Gerlach apparatus. An intense argon flash lamp light with the wavelength 170-230 nm was focused onto the polarized ${ }^{6} \mathrm{Li}$ atoms, giving polarized ionization photoelectrons that were injected into the accelerator. The accelerated beam energy ranged from 9 to $12 \mathrm{GeV}$. The average beam polarization, measured in the experimental area by the single arm Møller polarimeter, was $50 \%$, but the beam intensity was only around $5 \cdot 10^{8}$ electrons per pulse. The proton target was polarized, $50 \%$ on average, by dynamic nuclear orientation [59] using a butanol sample with a paramagnetic dopant. The scattered electrons were detected in a fixed $9^{\circ}$ angle spectrometer, and their momentum was determined. The experiment was repeated (as E130 [15]) by the same group in 1980 with higher beam energies of 16.2 and $22.7 \mathrm{GeV}$. The observed large $A_{1}^{p}$ asymmetries were in agreement with the expectations of the quark-parton model and its sum rules.

After completion of the early SLAC series of experiments, a new polarized program was developed at CERN. In a 1984-85 run the EMC collaboration extended the SLAC results, performing polarized muon-proton 
scattering. The $\mu^{+}$beam with intensity $4 \cdot 10^{7}$ particles per spill and with average energy $190 \mathrm{GeV}$ is naturally polarized. It originates from $\pi^{+}$decay, and its polarization was calculated to be $80 \%$. The target consisted of two cells filled with solid $\mathrm{NH}_{3}$ and polarized in opposite directions via dynamic nuclear polarization to $75 \%$ on average. The higher beam energy allowed measurements to obtain a much lower $x$ than the SLAC experiments. In the overlap region $x>0.1$ the results of both experiments agree well. However, The low $0.01<x<0.1$ data fall well below the theoretical prediction. As a consequence of this, the measured integral with the Regge type $g_{1}^{p}(x) \sim x^{0.12}$ extrapolation at low $x$, at $\left\langle Q^{2}\right\rangle=10 \mathrm{GeV}^{2}$,

$$
\left.\Gamma_{1}^{p}=0.126 \pm 0.010(\text { stat. }) \pm 0.015 \text { (syst. }\right)
$$

is in disagreement with the Ellis-Jaffe prediction by three standard deviations of the experimental error. With the assumption of isospin invariance and $\mathrm{SU}(3)_{\text {flavor }}$ symmetry in the baryon octet decays, i.e. with the value of axial charges $\Delta q_{3}$ and $\Delta q_{8}$ given in eqs. (1.118), (1.124), the $\Gamma_{1}^{p}$ result gives

$$
\Delta \Sigma=0.12 \pm 0.17
$$

The spin carried by the quarks was found to be surprisingly small, and this result became known as 'spin crisis'. It triggered a lot of theoretical activity and new experimental programs at CERN, SLAC and DESY.

The measurements at CERN continued, and the SMC collaboration took deuteron data [21] in 1992, 1994 and 1995, and proton data [20] in 1993 and 1996. The first year of running was devoted to the deuteron to have the first 
measurement of the neutron $g_{1}^{n}$ needed to test the Bjorken sum rule. The high energy muon beam extended the coverage down to $x=3 \times 10^{-3}$ at the high average $\left\langle Q^{2}\right\rangle$ of $10 \mathrm{GeV}^{2}$. However, the low intensity of the beam limited the statistical precision of the data. The results, in terms of integrals of structure functions, are quoted in table 1.2.

Table 1.2: Integrals of $g_{1}^{n, p, d}$ From CERN, SLAC and DESY Experiments.

\begin{tabular}{lcccr}
\hline Experiment & Target & $\begin{array}{c}\text { Target } \\
\text { Material }\end{array}$ & $\begin{array}{c}\left\langle Q^{2}\right\rangle \\
\left(\mathrm{GeV}^{2}\right)\end{array}$ & $\Gamma_{1} \pm$ stat. \pm syst. \\
\hline CERN EMC & $\mathrm{p}$ & ammonia & 10 & $0.126 \pm 0.010 \pm 0.015$ \\
CERN SMC & $\mathrm{p}$ & $\begin{array}{c}\text { butanol } \\
\text { ammonia }\end{array}$ & 10 & ${ }^{2} 0.149 \pm 0.006 \pm 0.010$ \\
CERN SMC & $\mathrm{d}$ & butanol & 10 & $0.041 \pm 0.006 \pm 0.005$ \\
SLAC E142 & $\mathrm{n}$ & ${ }^{3} \mathrm{He}$ & 2 & $-0.031 \pm 0.006 \pm 0.009$ \\
SLAC E143 & $\mathrm{p}$ & ammonia & 3 & $0.129 \pm 0.004 \pm 0.009$ \\
SLAC E143 & $\mathrm{d}$ & ammonia & 3 & $0.042 \pm 0.003 \pm 0.004$ \\
DESY HERMES & $\mathrm{n}$ & ${ }^{3} \mathrm{He}$ & 2.5 & $-0.037 \pm 0.013 \pm 0.008$ \\
\hline
\end{tabular}

Another series of SLAC experiments started with E142 [17] in the fall of 1992. A beam of high intensity was produced, typically $2 \cdot 10^{11}$ electrons per pulse, with three different energies 19, 23 and $26 \mathrm{GeV}$. The polarized electrons were obtained at the source by illuminating the AlGaAs photocathode with circularly polarized light. The polarization measured by the single arm Møller detector was about $36 \%$. The new method of obtaining the polarized beam of electrons allowed for a pseudo random choice of the beam helicity from pulse to pulse. This feature, unique to SLAC, resulted in strong suppression of the

\footnotetext{
${ }^{2} 1993$ and preliminary 1996 data combined [52].
} 
systematic effects related to the beam asymmetry. A high density ${ }^{3}$ He target was developed and polarized, to $33 \%$ on average, via spin-exchange optical pumping. About 300 million deep inelastic events were observed in two magnetic spectrometers centered at $4.5^{\circ}$ and $7^{\circ}$. The neutron asymmetries were measured directly for the first time, in the kinematic range of $0.03 \leq$ $x \leq 0.6,\left\langle Q^{2}\right\rangle=2 \mathrm{GeV}^{2}$, giving the most precise measurement of the neutron spin structure function $g_{1}^{n}(x)$ at that time. We note that for the first time the $A_{\perp}$ asymmetry was measured to minimize the systematic uncertainties.

Next came the winter of 1993/94 and SLAC experiment E143 [18, 19]. With the advent of the new strained GaAs photocathodes the beam polarization was $80 \%$. Also the beam energy was raised to $29 \mathrm{GeV}$. The spectrometer setup of E142 was used with new polarized at $65 \%$ ammonia (for proton) and polarized at $25 \%$ deuterated ammonia (for deuteron) targets. The data were also taken at the beam energies of 9 and $16 \mathrm{GeV}$ to study the $Q^{2}$ dependence [60]. The longitudinally and transversely [53] polarized proton and deuteron structure functions were measured in the kinematic range of $0.03 \leq x \leq 0.7$ and the average $\left\langle Q^{2}\right\rangle$ of $3 \mathrm{GeV}^{2}$.

A new spin structure program HERMES [22] was started recently at the DESY collider. It utilizes a $28 \mathrm{GeV}$ beam of positrons in the HERA storage ring polarized to an average $55 \%$ by the Sokolov-Ternov mechanism [61]. The polarized ${ }^{3}$ He atoms, $46 \%$ on average, were injected directly into the windowless cell located inside the positron ring allowing for almost no dilution at the target. The $g_{1}^{n}$ structure function was measured in the kinematic range 


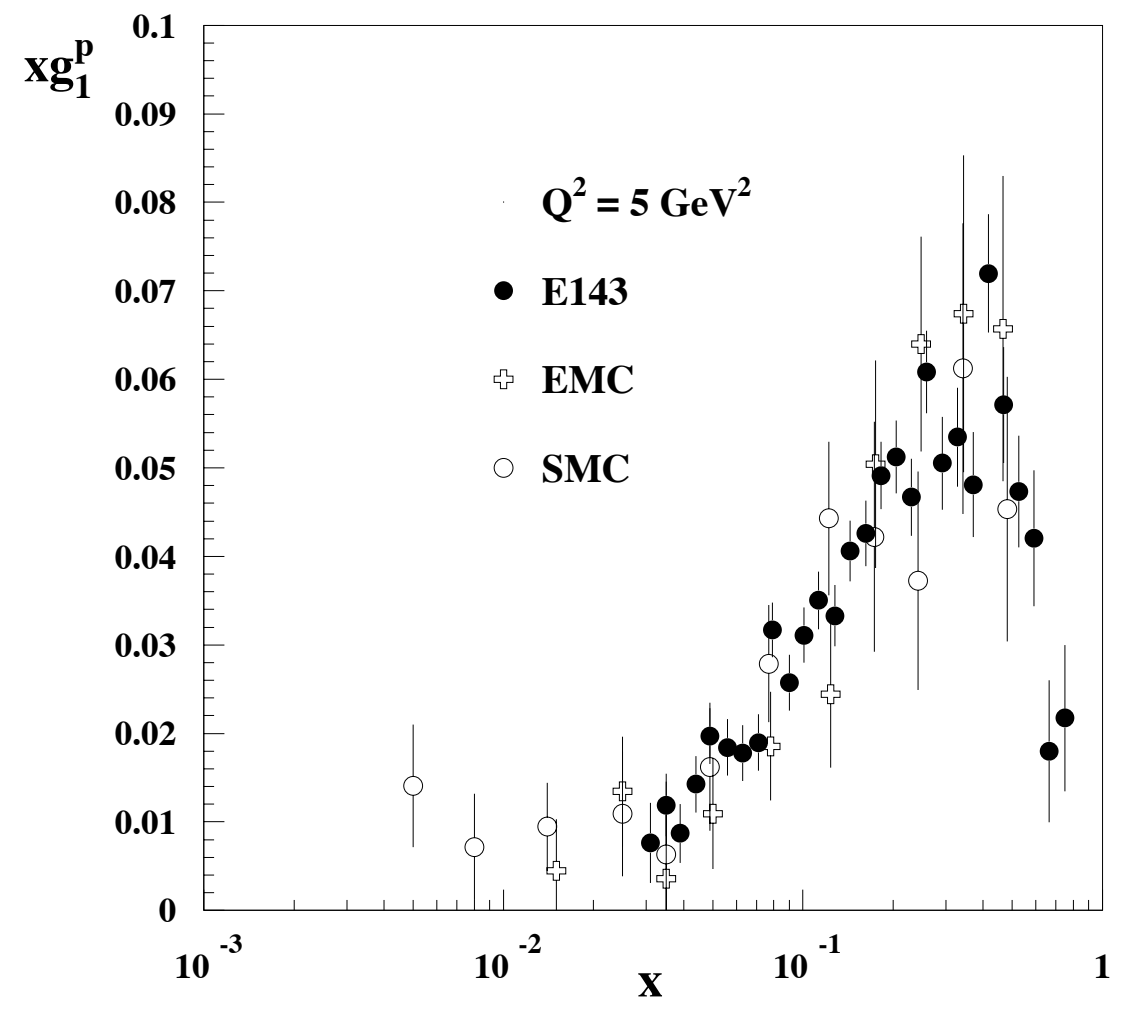

Figure 1.2: Proton data for $x g_{1}^{p}(x)$.

$0.02 \leq x \leq 0.6,\left\langle Q^{2}\right\rangle=2.5 \mathrm{GeV}^{2}$. We note that the HERMES possibility of tagging the leading hadron (so called semi inclusive scattering) allows one to probe the valence and sea distributions directly. This program will continue beyond the year 2000 .

The results of these experiments for the $x g_{1}^{p}$ and $x g_{1}^{n}$ structure functions are presented in figs. 1.2 and 1.3. We see that the proton data are positive, relatively large and rather well established in the common $x$ region of experiments. At $x \lesssim 0.01$ the data is sparse, and we have to rely entirely on models for extrapolation to $x=0$. Note that the lowest $x$ point for $x g_{1}^{p}$ 


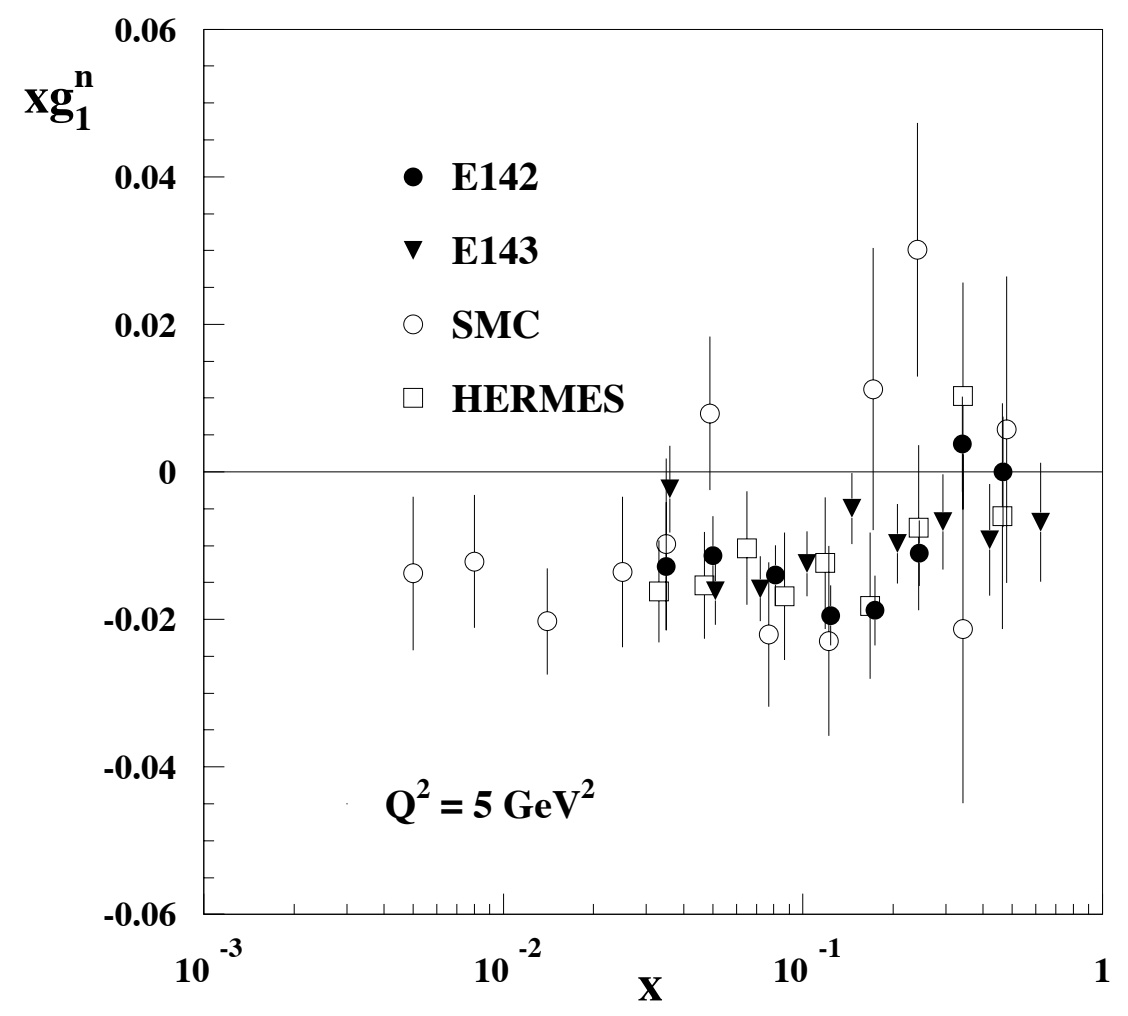

Figure 1.3: Neutron data for $x g_{1}^{n}(x)$.

suggests a rise, which would mean that $g_{1}^{p}(x)$ is more divergent than $1 / x$ and thus nonintegrable. This measurement comes from the 1993 run of SMC. The preliminary analysis of the 1996 run [52] lowers that point to a value consistent with zero. The neutron values of $x g_{1}^{n}$ are negative and relatively small. The experimental data are consistent in the region of overlapping $x$, but the errors are still significant in comparison with the values. And, as in the proton case, behavior of $x g_{1}^{n}$ for $x \lesssim 0.01$ remains unconstrained.

The traditional analysis of all data existing at the time was performed by Ellis and Karliner [62] in 1995. The of integrals of $g_{1}$ 's given by the different 
experiments were evolved to the same $Q^{2}$ of $3 \mathrm{GeV}^{2}$ and then combined to find the Bjorken sum rule

$$
\Gamma_{1}^{p}-\Gamma_{1}^{n}=0.164 \pm 0.011 \quad \text { at } 3 \mathrm{GeV}^{2}
$$

in agreement with the prediction. The authors go further and use the Bjorken sum rule to extract $\alpha_{s}\left(3 \mathrm{GeV}^{2}\right)=0.328_{-0.037}^{+0.026}$, see eq. (1.122), or equivalently,

$$
\alpha_{s}\left(M_{Z}^{2}\right)=0.119_{-0.005}^{+0.003}
$$

They also make a global fit and obtain the flavor decomposition of the nucleon spin,

$$
\begin{aligned}
& \Delta u=0.82 \pm 0.03 \\
& \Delta d=-0.44 \pm 0.03 \\
& \Delta s=-0.11 \pm 0.03
\end{aligned}
$$

and

$$
\Delta \Sigma=0.27 \pm 0.04
$$

One should keep in mind the $Q^{2}$ evolution assumption, the uncertainty of the low $x$ extrapolation assumptions, and the neglect of higher twist contributions and higher order corrections in the analysis. 


\section{CHAPTER 2}

\section{EXPERIMENTAL APPARATUS}

The SLAC E154 experiment collected data for two months during OctoberNovember 1995.

The polarized electrons were produced at the source, accelerated in $3.2 \mathrm{~km}$ of linac to $48.3 \mathrm{GeV}$ and delivered, longitudinally polarized, to the experimental area of the End Station A. The Møller detector determined that the beam polarization was $82 \%$. The electrons scattered off the optically polarized, $38 \%$ on average, ${ }^{3}$ He target and were detected in two independent magnetic spectrometers positioned at the central angles $2.75^{\circ}$ and $5.5^{\circ}$ with respect to the beam line. Each spectrometer consisted of Cherenkov detectors, hodoscopes and an electromagnetic calorimeter. The gas threshold Cherenkov detectors were used for particle identification. Several planes of scintillator hodoscopes served for tracking particles, allowing the determination of the direction and the value of the electron momentum. The lead 
glass calorimeter measured the deposited energy of a scattered particle. The kinematic range of the results on $g_{1}^{n}(x)$ and $g_{2}^{n}(x)$ determined from these measurements was $0.014 \leq x \leq 0.7$ and $1 \mathrm{GeV}^{2} \leq \mathrm{Q}^{2} \leq 17 \mathrm{GeV}^{2}$ (average $\left\langle Q^{2}\right\rangle=5\left(\mathrm{GeV}^{2}\right)$

The experiment was an effort of 81 physicists representing 23 institutions.

\subsection{SLAC polarized source}

Since 1992 both the SLAC accelerator and SLC have been operating solely with polarized electrons. The electrons are produced at the source [63, 64, 65] with $120 \mathrm{~Hz}$ frequency. For the fixed target experiments the first 119 pulses, which are $10 \mu$ sec long, are produced by a flashlamp pumped titanium-sapphire (Ti:S) dye laser operating at $850 \mathrm{~nm}$ wavelength. The last witness pulse, used for monitoring purposes, only lasts $2 \mathrm{~ns}$ and is generated by another YAG-pumped Ti:S laser. The schematic view of the source is shown in fig. 2.1. The linearly polarized light goes through the slicer and shaper (laser pulse chopper) where it is cut to $240 \mathrm{~ns}$ and shaped slightly non-uniformly to compensate for beam loading effects of the linac. Next it is circularly polarized via a system of two Pockels cells with axes rotated $45^{\circ}$ with respect to each other. The first cell operates at its quarterwave voltage, and the second one at zero, with small corrections generated by the feedback loop to maintain the charge asymmetry below $10^{-4}$. The positive quarterwave HV produces one helicity, while the negative HV pulse produces the opposite helicity. The sign of the applied HV, and thus the helicity, 


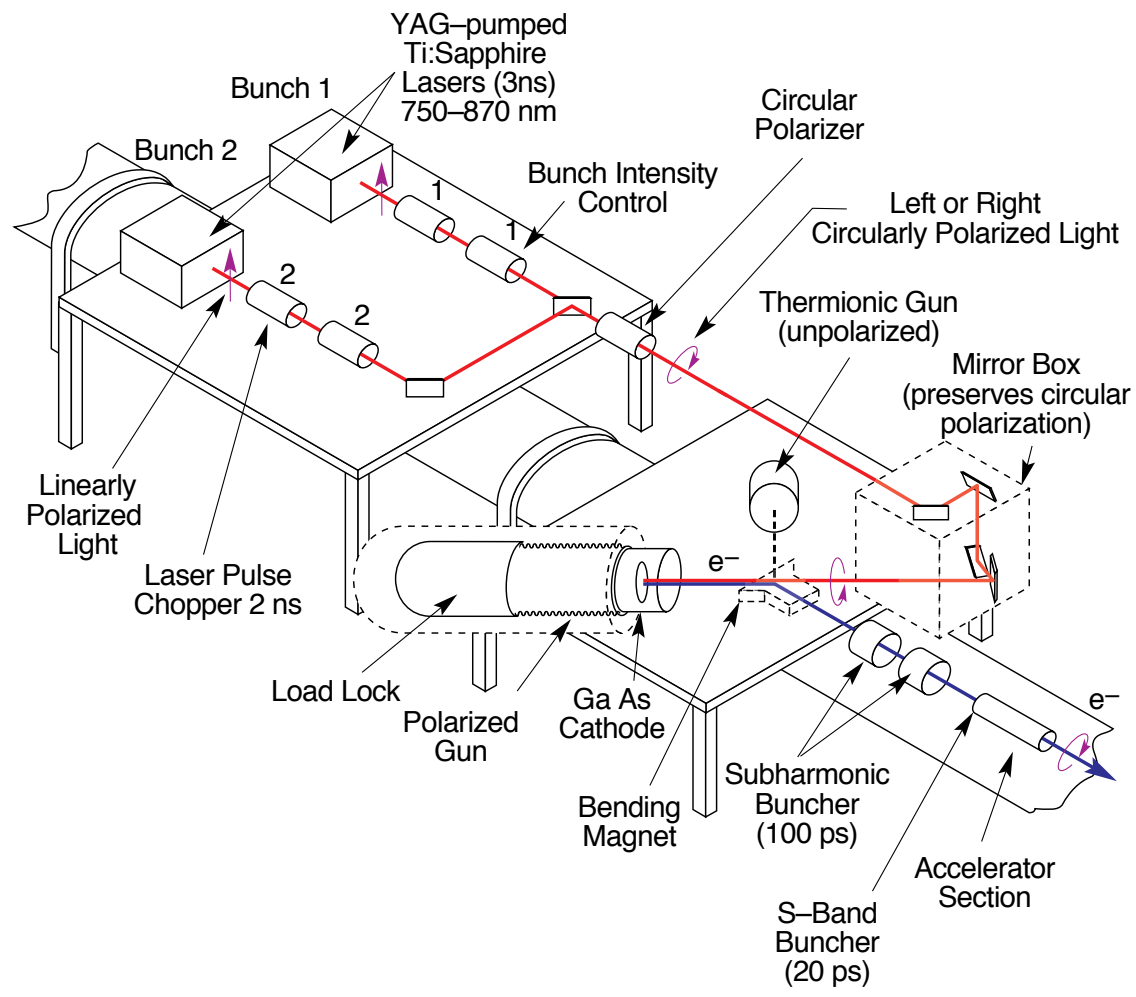

Figure 2.1: SLAC polarized source setup for SLC runs. For E154 operation one of the lasers was replaced by a flashlamp pumped Ti:S laser.

follows a pseudo-random pattern from pulse to pulse, a unique feature of the SLAC accelerator which reduces the false asymmetries correlated with the beam helicity. The photons with a well defined helicity hit the cathode of the electron gun, photoemitting polarized electrons.

Several types of gallium arsenide related cathodes have been tried. The E142 experiment used $\mathrm{Al}_{0.12} \mathrm{Ga}_{0.88} \mathrm{As}$ material. The filled top valence band of $\mathrm{AlGaAs}$ has $\mathrm{P}_{3 / 2}$ symmetry, while the empty conduction band has $\mathrm{S}_{1 / 2}$ symmetry. The addition of $\mathrm{Al}$ changes the energy gap to $1.63 \mathrm{eV}$ and makes 
it easier to tune the frequency of lasers to the gap. The light of +1 helicity excites electrons from $\left|P_{3 / 2}\left(m_{j}=-3 / 2\right)\right\rangle$ to $\left|S_{1 / 2}\left(m_{j}=-1 / 2\right)\right\rangle$ with probability 3 times larger than the competing transition from $\left|P_{3 / 2}\left(m_{j}=-1 / 2\right)\right\rangle$ to $\left|S_{1 / 2}\left(m_{j}=+1 / 2\right)\right\rangle$, giving the theoretical $50 \%$ polarization limit of the emitted electrons. In practice polarizations on the level of $40 \%$ were achieved. To get the electrons from the conduction band to the vacuum one needs to pass the work function barrier, approximately $4 \mathrm{eV}$ for pure GaAs. By deposition of cesium and an oxidizer $\left(\mathrm{O}_{2}\right.$ or $\left.\mathrm{NF}_{3}\right)$ the potential of the vacuum can be lowered below the conduction band potential. A material with such characteristic is said to have a negative affinity. Now the conducting electrons can tunnel through the potential barrier at the surface out of the material guided by $60 \mathrm{kV}$ applied to the cathode. During the experiment the cesium layer gets slowly deactivated, probably due to the residual gas in the vacuum system, making the penetration of the surface barrier more difficult. There is a drop in the quantum efficiency of the cathode, since only electrons closer to the surface can escape. These electrons are less subject to latice interactions on the way out. That produces the observed increase in the polarization.

For our experiment a strained GaAs material for the cathode was used. This is achieved by growing a thin (e.g. $0.25 \mu \mathrm{m}$ ) layer of GaAs on a thick layer of $\mathrm{GaAs}_{1-x} \mathrm{P}_{x}$ (e.g. $\left.25 \mu \mathrm{m}\right)$. The $x$ fraction of replaced As atoms was varied smoothly from 0 to $28 \%$. The lattice constant of the phosphorus doped GaAs is $1 \%$ smaller than pure GaAs. That produces a compression in GaAs lattice and breaks the degeneracy between the $\left|P_{3 / 2}\left(m_{j}= \pm 3 / 2\right)\right\rangle$ and 
$\left|P_{3 / 2}\left(m_{j}= \pm 1 / 2\right)\right\rangle$ valence band levels producing $50 \mathrm{mV}$ energy difference. In figure 2.2 we show the energy structure of strained GaAs. The theoretical

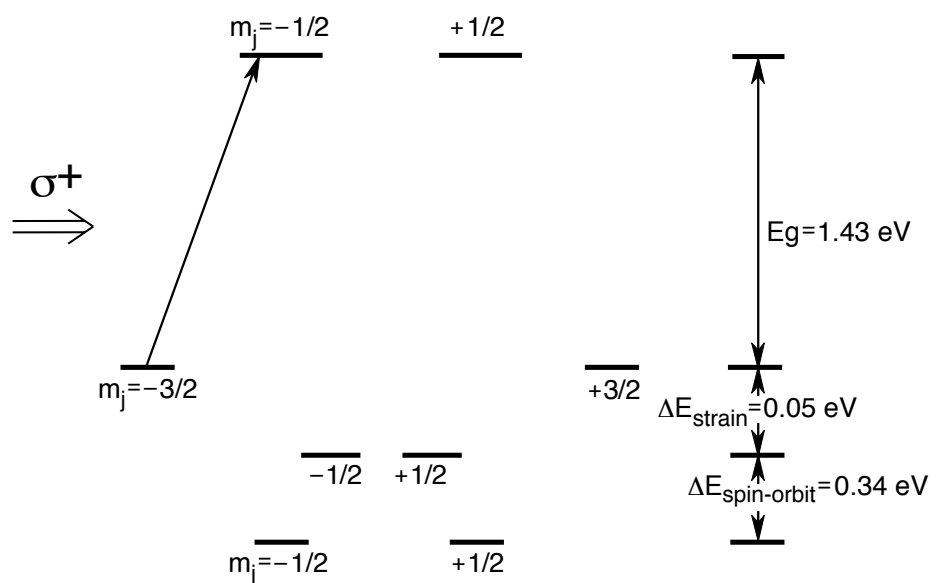

Figure 2.2: The energy level structure of strained GaAs.

limit for the polarization of photo-emitted electrons is $100 \%$. In practice polarizations above $80 \%$ were achieved. The level splitting is not large enough to allow tuning of the laser frequency completely away from the energy of the competing transition. Furthermore, even $99.5 \%$ circularly polarized light has $10 \%$ linearly polarized component. There is also some strain relaxation, and thermally excited electrons in the conduction band contribute to the lower than theoretical limit polarization.

The $0.5-2 \times 10^{11}$ photoemitted electrons were bunched together and sent down the accelerator. 


\subsection{Beam acceleration and transport}

The $3.2 \mathrm{~km}$ of the linear accelerator consists of 30 sectors. Each sector contains steering magnets: dipoles and a quadrupole, some elements for monitoring beam position and current, and, obove all, eight klystrons, each accompanied by a system of RF resonant cavities, the so called SLAC Energy Doubler (SLED). Before our experiment, when the SLED technique was not implemented, a beam energy of $29 \mathrm{GeV}$ was achieved.

Every klystron feeds microwave radiation at $2856 \mathrm{MHz}$ into copper cavities. The fields in the cavities build up, and a wave of increasing amplitude is emitted from the coupling apertures of each cavity. At $0.8 \mu$ sec before the end of a $3.5 \mu$ sec long RF pulse, the $180^{\circ}$ phase shifter reverses the phase of the output wave from the klystron [66]. The emitted and reversed waves add in phase at the accelerator. Immediately after the phase reversal the amplitude of the superposition is twice the amplitude of each single wave. That allows for the maximum measured unloaded energy of $55 \mathrm{GeV}$ and maximum practical beam energy up to $52 \mathrm{GeV}$. This setup can only accelerate about a $150 \mathrm{~ns}$ beam pulse within an energy spread of $0.5 \%$ with $10^{11}$ electrons. By applying two additional $180^{\circ}$ phase inversions for $20 \%$ of klystrons [67], the classical SLED pulse is flattened to achieve an energy spread of $0.5 \%$ over 240 ns.

The electrons are transported from the linac to End Station A through the A-Line, which had to be upgraded to handle beam with energies up to $50 \mathrm{GeV}$. The E154 experiment was the first after the modification. The 
48.75 $\mathrm{GeV}$ electrons from the linac are first bent over $0.5^{\circ}$ by a set of five pulsed and two DC magnets that offer the option of switching the beam between four beam lines on a pulse-by-pulse basis [68]. This area is called the Beam Switchyard. The next $24^{\circ}$ bend, for the total of $24.5^{\circ}$, is provided by twelve identical dipole magnets. Nine quadrupoles are used to control the beam divergence and the spot size at the target. Two massive high-power collimators limit the energy spread of the beam.

\subsection{Beam parameters and monitoring}

\subsubsection{Energy}

The beam energy in the experimental area of the End Station A (ESA) was established in two independent ways.

The first uses the optics of the A-Line [68]. Twelve dipole magnets were connected in series with an identical, off line dipole equipped with a flip-coil. The current induced in the flip-coil measures the magnetic field of the dipoles which, with the knowledge of the bending angle, determines the momentum of the electrons to about $0.1 \%$. Some corrections to the flip-coil measurement are needed: for the synchrotron radiation loss, stray magnetic field beyond the coil, a bias in the coil readout electronics and the steel support plate present in all the beam line magnets except in the reference magnet. Including the corrections, the energy in the ESA was determined as $48.325 \mathrm{GeV}$ with an accuracy of $40 \mathrm{MeV}$. 
The second measurement was done by the energy scan of the longitudinal beam polarization in the Møller detector. The details are given in the next section 2.4.6 (page 60). This determination gave the energy in the ESA equal to $48.30 \mathrm{GeV}$, in excellent agreement with the first.

The value $\mathrm{E}$ of the energy was monitored throughout the experiment via the flip-coil signal. The energy spread of the beam $\Delta E / E$ was restricted to $1 \%$ by the A-line collimators.

\subsubsection{Current}

The beam current was measured for each pulse with two independent ferromagnetic toroids located more than $30 \mathrm{~m}$ apart, upstream from the target. The beam passing through the ferrite core of the toroid induced a signal in the loops of wire wrapped around the core, which in turn was sent to a resonant LC circuit. The induced current was then amplified and integrated. The total charge is proportional to the beam current. Several times a day a precisely charged capacitor was discharged through each of the toroids, giving the calibration coefficients for the beam measurements. In this way the total beam charge was established with about $0.5 \%$ accuracy.

\subsubsection{Position and steering}

The quick coarse centering and focusing of the beam was made with two mylar roller screens, coated with fluorescent $\mathrm{ZnS}$. The screens, one in front of the target and the second $25 \mathrm{~m}$ upstream, were moved into the beam, observed with television monitors, and then moved back before collecting the 
data. They were placed periodically in the beam for monitoring purposes. The main information about the beam position and width was obtained from a wire array located $10.5 \mathrm{~m}$ downstream of the target. There were 24 wires in the $x$ direction and 24 in the $y$ direction, made of $0.127 \mathrm{~mm}$ diameter CuBe wire and spaced $1.1 \mathrm{~mm}$ apart. Each pulse of current induced in a single wire by the beam was integrated by ADC's, read out by our Data Acquisition System (DAQ) and logged on tape. The information was also available to the linac operators at the Main Control Center (MCC) for minor adjustments and monitoring.

Additional beam quality monitoring was performed with the use of two scintillation counters. The first was positioned upstream from the target in the ESA alcove, close to the beamline. It was sensitive to the beam scraping and thus dubbed 'bad spill monitor'. The other counter, the 'good spill monitor', was downstream from the target, several meters away from the beampipe. It was sensitive to particles created at the target. Because its signal (and also the signal from the first counter) was displayed on the oscilloscope (both in the Counting House and MCC) it was a sensitive indicator of the time structure of the beam. The ADC integrated signals were also collected by DAQ and stored on tape for the analysis.

Other monitors of the beam included two traveling wave beam position monitors (TWBPM) and a secondary emission monitor (SEM) for target protection. Traveling wave BPM's were located in front of the target. One microwave cavity produced an RF signal proportional to the horizontal devi- 
ation of the beam from the cavity center. The second was sensitive to vertical displacements.

The SEM consisted of a thin aluminum foil with a $2 \mathrm{~cm}$ circular aperture

for the beam. The opening matched the diameter of the target. Thus, large signals from the monitor indicated a potentially destructive displacement of the beam and triggered an automatic beam shutdown. This prevented the beam from hitting the thick side walls of the target.

\subsubsection{Spin precession}

The helicity of electrons entering the linac is the same as at the source, and thus the same as the helicity of the photons hitting the photocathode. It remains unchanged until the entrance to the A-line. In the A-line the beam is bent by $\theta_{\text {bend }}=24.5^{\circ}$, and the spins of electrons undergo a precession [69]

$$
\theta_{\text {prec }}=\gamma\left(\frac{g-2}{2}\right) \theta_{b e n d}
$$

relative to the beam direction. Here $\gamma=E / m$, and $g=2.00231 \ldots[70]$ is the gyromagnetic ratio of the electron. For the full A-line bend the above formula can be interpreted as follows: The electron helicity is flipped every $3.2374 \mathrm{GeV}$ of beam energy. The E154 experiment was set to run at 48.56 GeV linac energy. That corresponds to $\theta_{\text {prec }}=15 \pi$. The helicity of the electrons in the End Station A was opposite to that at the source.

The A-line energy is modified due to synchrotron radiation, which, being proportional to $E^{4}$, becomes important at high energies. The synchrotron 
energy loss $\Delta E$ is given by [71]

$$
\Delta E=\frac{2}{3} \frac{e^{2}}{\rho} \beta^{3}\left(\frac{E}{m}\right)^{4} \theta_{\text {bend }},
$$

where $\rho=85.927 \mathrm{~m}[72]$ is the bending radius in meters, $\theta_{\text {bend }}$ is expressed in radians and $\beta \approx 1$ for our energy. Taking into account this loss the End Station A beam energy $E=48.362 \mathrm{GeV}$ corresponds to $15 \pi$ spin rotations. The measured energy was $E=48.325 \pm 0.040 \mathrm{GeV}$, as explained in section 2.3.1.

The synchrotron radiation also increases the beam emittance, resulting in a larger spot size at the target. To reduce this effect and bring the beam diameter back to the required $\sim 1 \mathrm{~mm}$ at the target, a quadrupole magnet (Q41) was installed at the entrance to the ESA with a focal point at the target.

\subsubsection{Helicity reversal}

The reversal of the beam polarization on a pulse-to-pulse basis with the Pockels cell was very important for reducing systematic errors. Possible false asymmetries arising from slow changes in spectrometer acceptance were averaged out by the rapid beam helicity reversal. Also, by changing the target polarization direction, we could further average asymmetries due to possible helicity related differences in beam properties. The absolute helicity of the beam in the ESA was determined by the sign of the asymmetry measured by the Møller polarimeter. 
The beam helicity was chosen on a pulse-to-pulse basis using a pseudorandom number generator. The seed began with the choice of a 32 bit random number. The last bit, the 32nd, determined the helicity of the beam. For the next pulse, the 19 th and 32 nd bits were subject to a logical XOR operation. After the remaining bits had been shifted up, the result was placed as the 1-st bit. The new 32 nd bit determined the helicity of this pulse. Then the cycle was repeated. The generator ran continuously with the source on or off. Thus, knowledge of the polarization bits within the 33 consecutive pulses was needed to determine the random sequence seed. Then the prediction for the polarization of each spill could be made based on the spill number and could be used to test the integrity of the system.

The polarization state measurements were sent to the Counting House via four physically distinct paths and then recorded by the Data Acquisition System. They were referred to as the PMON Line, Mach Line, HV Line (short for the Pockels Cell High Voltage Line) and Veto Line (short for the Klystron Veto Module Line). Each line delivered two bits. The combination '01' referred to the positive helicity photons incident on the photocathode, while ' 10 ' to the negative helicity. The ' 00 ' result meant that the unpolarized beam and '11' indicated an error. Typically all four measurements agreed with the prediction [73] and if they did not, the spill was discarded. If the rate of disagreement exceeded $5 \cdot 10^{-4}$ for a run, the entire run was excluded from the analysis. 


\subsection{Møller polarimeter}

A single arm Møller polarimeter was used throughout the experiment to measure the beam polarization. It consisted of polarized ferromagnetic target foils, a momentum-analyzing dipole magnet and five silicon detectors. It was also used to optimize the wavelength of the source laser with respect to the beam polarization and to calibrate the flip coil energy (see also section 2.3.1 on page 47$)$.

\subsubsection{Møller asymmetry}

The cross section for the Møller scattering of polarized $\mathrm{e}^{-}$on polarized $\mathrm{e}^{-}$ has the form

$$
\frac{d \sigma}{d \Omega}=\frac{\alpha^{2}}{s} \frac{\left(3+\cos ^{2} \theta\right)^{2}}{\sin ^{4} \theta}\left[1-P_{z}^{B} P_{z}^{T} A_{z z}(\theta)\right]
$$

in the CM frame [74], where $s$ is the total CM momentum squared. The z-axis is along the beam direction, and $\theta$ is the CM scattering angle. Here the longitudinal beam and target polarizations are denoted by $P_{z}^{B}$ and $P_{z}^{T}$, respectively, and the asymmetry $A_{z z}(\theta)$ is given by

$$
A_{z z}(\theta)=\frac{7+\cos ^{2} \theta}{\left(3+\cos ^{2} \theta\right)^{2}} \sin ^{2} \theta
$$

By counting the rates for the electron beam and target spins aligned, $\sigma^{\uparrow \uparrow}$, and antialigned, $\sigma^{\uparrow \downarrow}$, we can measure the asymmetry

$$
A(\theta)=\frac{\sigma^{\uparrow \downarrow}-\sigma^{\uparrow \uparrow}}{\sigma^{\uparrow \downarrow}+\sigma^{\uparrow \uparrow}}=P_{z}^{B} P_{z}^{T} A_{z z}(\theta)
$$


If the target polarization $P_{z}^{T}$ is known independently, the above formula can be used to determine the beam polarization $P_{z}^{B}$. The asymmetry has a maximum at $\theta=90^{\circ}$, where the unpolarized laboratory cross section is $0.179 \mathrm{~b} / \mathrm{sr}$ and $A_{z z}=7 / 9$. With a typical target foil polarization of 0.08 and the beam polarization of 0.80 , the expected measured asymmetry $A\left(90^{\circ}\right)$ is roughly $0.05[75]$.

\subsubsection{Polarimeter design}

The design was an evolution of previous End Station A Møller polarimeters [76] and utilized many ideas and components of previous designs.

The E154 Polarimeter consisted of polarized target foils, a mask to define the azimuthal and vertical acceptance, a magnet to momentum analyze the scattered electrons, and detectors to measure the scattering rate. It was located upstream from the ${ }^{3}$ He target. Schematic top and side views are shown in fig. 2.3. Six target foils were installed at a $20.7^{\circ}$ angle to the beamline [75]. The foils were 20(2 of them), 30, 40(2) and $154 \mu \mathrm{m}$ thick and made

of ferromagnetic Vacoflux (49\% Fe, $49 \%$ Co, $2 \%$ Va by weight). They were magnetized to near saturation by Helmholtz coils providing nearly $100 \mathrm{G}$ in the target center. The mask was made from 25 radiation lengths of tungsten. It had a central hole for the unscattered beam and two wedge-shaped holes to select vertically scattered electrons. That gave the $\phi$ acceptance of 0.20 $\mathrm{rad}$ (top hole) and $0.22 \mathrm{rad}$ (bottom) over the range of allowed scattering angles, $3.59<\theta_{\text {lab }}<8.96 \mathrm{mrad}$ in the lab frame. Additional $\mathrm{Pb}$ shielding and a collimator prevented the single-scattered target photons from reaching 
Figure 2.3: Top (top picture) and side (bottom picture) views of the E-154 Møller Polarimeter.

the detectors. The momentum selection of the scattered electrons was accomplished with a dipole magnet producing $\int B d l 33 \mathrm{kG}-\mathrm{m}$ at 900 A current. 
The electrons were observed by five silicon detectors mounted $28.9 \mathrm{~m}$ from the target. The top detector had 48 channels with a fine segmentation of $2.18 \mathrm{~mm}$ in the vertical $(\theta)$ direction. It was mounted on a remotely controlled stage and could be positioned anywhere within the Møller acceptance. Typically the Møller peak corresponded to $\theta=94^{\circ}$ in the CM. Each of the four bottom detectors had 12 channels with a coarser separation of $8.69 \mathrm{~mm}$. They covered the $\theta \mathrm{CM}$ scattering angle range of $93^{\circ}-104^{\circ}$. The silicon channels were connected to charge sensitive preamplifiers and then to ADC's read by DAQ. The unscattered electrons continued to travel the beam pipe that went through the magnet. The beam pipe was enclosed in the iron septum to eliminate the magnetic field.

\subsubsection{Foil polarization measurement}

The targets were made of Vacoflux, as mentioned above, composed of $49 \%$ Fe, $49 \%$ Co and $2 \%$ Va. The foils were typically $3 \mathrm{~cm}$ wide by $35 \mathrm{~cm}$ long by $20-154 \mu \mathrm{m}$ thick. They were polarized to saturation by a $100 \mathrm{G}$ magnetic

field. Only two electrons in $\mathrm{Fe}$ and $\mathrm{Va}$ contribute to the magnetization, and on average the electrons were $\sim 8 \%$ polarized. This polarization was determined measuring the magnetization $M[77]$. An integrating voltmeter was connected to a pick-up coil made of $N=500$ turns wrapped around the foil. The bipolar Helmholtz coil power supply ramped the $H$ field from -100 to +100 Gauss, and the voltmeter recorded the flux change

$$
\Phi_{\text {foil in }} \equiv \int V(t) d t=2 N\left[A_{f} B+\left(A_{c}-A_{f}\right) H\right]
$$


where $A_{c}$ and $A_{f}$ are the cross-sectional areas of the pick-up coil and the foil respectively. The induction $B$ at the coil has contributions from the magnetic field $H$ and the magnetization $M$ in accordance with

$$
B=H+4 \pi M
$$

so that the flux $\Phi_{\text {foil in }}$ can be re-written as

$$
\Phi_{\text {foil in }}=2 N\left(A_{c} H+4 \pi A_{f} M\right) .
$$

Because the foils were very thin, the $H$ field was almost unaffected by their presence. Repeating the procedure without the foils in place,

$$
\Phi_{\text {foil out }}=2 N A_{c} H
$$

was measured. The magnetization $M$ is given by

$$
M=\frac{\Phi_{\text {foil in }}-\Phi_{\text {foil out }}}{8 \pi N A_{f}} .
$$

The magnetization $M$ has to be corrected for the orbital angular momentum contribution to the electron polarization. Let us recall the definition of $g^{\prime}$, the magneto-mechanical ratio [78], $M=g^{\prime} \mu_{B}(L+S)$. The spin contributes $M_{S}=g \mu_{B} S$ to $M$, and the orbital angular momentum contributes $M_{L}=\mu_{B} L$. Obviously, $M=M_{S}+M_{L}$. The equations can be solved for $M_{S}$ in terms of $M$, i.e. for the fraction of the magnetization due to the electron polarization, and the electron polarization $P^{T}=M_{S} /\left(n_{e} \mu_{B}\right)$, where $n_{e}$ is the electron density, can be computed using

$$
P^{T}=\left(\frac{g^{\prime}-1}{g^{\prime}}\right)\left(\frac{g}{g-1}\right) \frac{M}{n_{e} \mu_{B}} .
$$


The gyromagnetic ratio $g=2.00231 \ldots[70]$. The $g^{\prime}$ ratio was measured for the alloy consisting of $50 \% \mathrm{Fe}$ and $50 \%$ Co to be $g^{\prime}=1.916 \pm 0.002[79]$. We assume that it could only be slightly affected by the presence of $2 \% \mathrm{Va}$ and increase the error to \pm 0.02 . The polarization of the foils was measured twice, before and after the experiment, giving the same results within $0.1 \%$ on average. The measured average polarization of the foils was $8.2 \%$ with relative error $1.7 \%[75]$.

\subsubsection{Beam polarization results}

The Møller data were taken during about 140 special, dedicated runs in the E154 experiment. The runs were typically ten minutes long. The preamplified outputs of the silicon detector channels were integrated by ADC's and recorded by the Data Acquisition System. In the analysis stage the averages for each ADC channel were made separately for pulses with left (L) and right $(\mathrm{R})$ beam helicities. Then the sums $(\mathrm{R}+\mathrm{L})$ and differences $(\mathrm{R}-\mathrm{L})$ were formed. The background under the unpolarized $(\mathrm{R}+\mathrm{L})$ Møller scatters was estimated by fitting the $(\mathrm{R}+\mathrm{L})$ line-shape to an arbitrary quadratic background plus the line-shape expected from the unpolarized Møller scattering. The technique for estimating the unpolarized line-shape was based on the measured polarized (R-L) line shape with corrections for the atomic motion of the target electrons. It is in excellent agreement with Monte Carlo results [80]. The atomic motion correction comes from the atomic momentum of unpolarized electrons inside Fe and Co atoms. Although this momentum $p$ is only on the order of $100 \mathrm{KeV}$, it considerably smears $(\sim 10 \%)$ the lab 
frame scattering angle by a maximum amount [81]

$$
\tan \left(\theta_{\text {smeared }}\right) \approx \tan (\theta) \sqrt{1-\frac{p}{m}},
$$

because it is to be compared with the electron mass $m$ and not with the beam energy. The elastic peak from the unpolarized inner shell electrons is significantly wider than the peak from the polarized electrons. Experimentally the consequences of this effect depend on how much of the Møller

peak is detected. As the fraction of the detected peak gets smaller, the measured asymmetry gets larger since the relative contribution of events from the unpolarized electrons decreases.

The measured asymmetry was calculated from the ADC averages as

$$
A=\frac{\sum_{i}(R-L)_{i}}{\sum_{i}(R+L)_{i}-\sum_{i}(\text { background })_{i}},
$$

where the sum is over channels including the Møller peak. The sum range was chosen large enough to avoid the necessity of additional corrections for the target motion effect. The background subtraction increased the measured asymmetry by $17 \%-24 \%$ relative.

The beam polarization history is given in table 2.1 and is shown in figure 2.4. The statistical errors for each run were typically $0.003-0.006$. The average beam polarization (weighted by statistics of $A_{\|}$) for the experiment was $81.8 \% \pm 2.3 \%$.

\subsubsection{Systematic uncertainties}

The small statistical relative errors of $0.4 \%-0.7 \%$ per run made possible precise studies of the systematic errors. The overall systematic error has 
Table 2.1: Measured Longitudinal Beam Polarization For Different Running Periods. The Error is Statistical Only.

\begin{tabular}{cc}
\hline Run range & Polarization \\
\hline $1329-1411$ & $0.759 \pm 0.004$ \\
$1456-1684$ & $0.775 \pm 0.005$ \\
$1691-2311$ & $0.814 \pm 0.002$ \\
$2316-3371$ & $0.824 \pm 0.001$ \\
$3377-3788$ & $0.826 \pm 0.002$ \\
\hline
\end{tabular}

contributions from the foil polarization due to uncertainty of the $g^{\prime}$ factor, uncertainties in the expected Møller asymmetry for each detector, and uncertainties in the background subtraction. The sizes of various contributions are given in table 2.2 . The helicity related correlations were cancelled out Table 2.2: Relative Systematic Error Contributions to the Beam Polarization Measurement.

\begin{tabular}{ll}
\hline Systematic error contribution & Value \\
\hline Foil magnetization & $1.9 \%$ \\
Kinematic acceptance & $0.3 \%$ \\
Background correction & $2.0 \%$ \\
Fit range & $0.3 \%$ \\
TOTAL & $2.8 \%$ \\
\hline
\end{tabular}

by pulse-by-pulse changes of the beam helicity and by almost always taking runs in pairs with opposite target helicities.

\subsubsection{Beam energy scan and beam helicity determina- tion}

The Møller polarimeter permitted an independent measurement of the beam energy [75]. The electron spin precesses at the A-line bend by an 


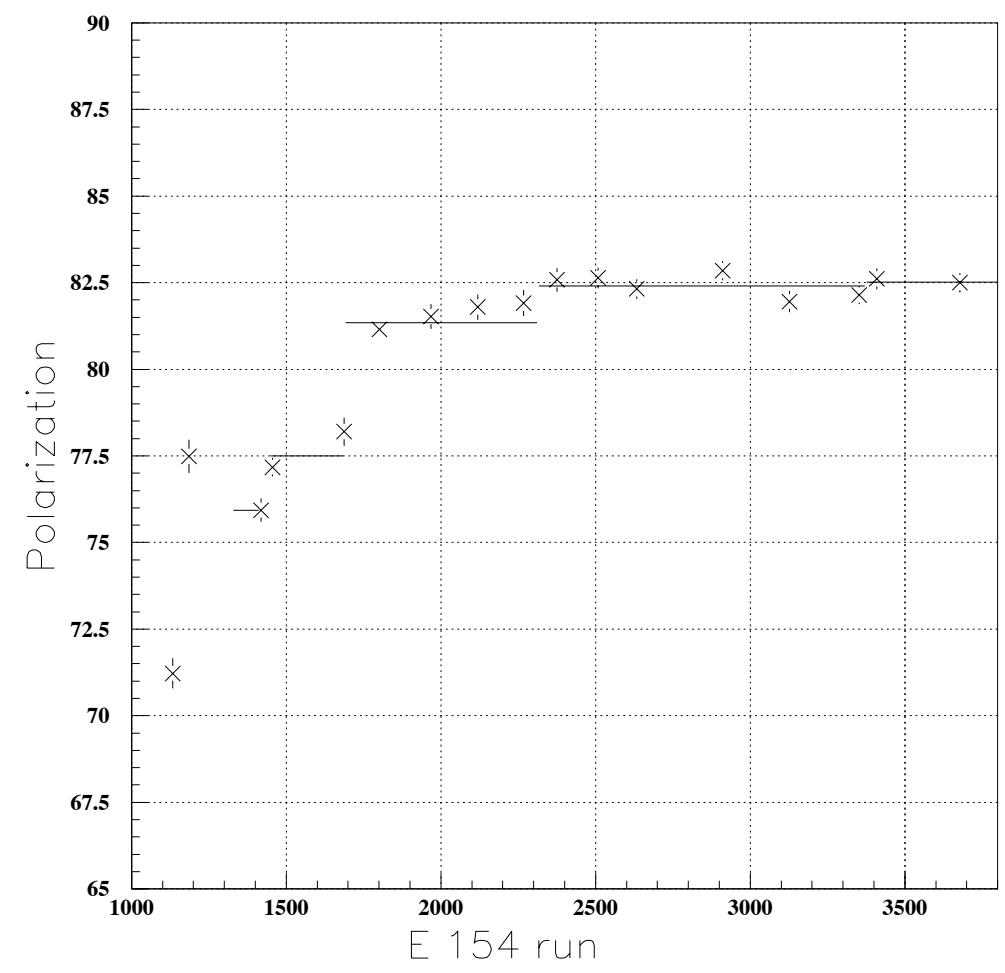

Figure 2.4: Measured longitudinal beam polarization versus E154 run number. The errors are statistical only. Changes in the measured average value of polarization indicate various adjustments made to the source during the run.

amount given by (g-2) and the beam energy E (see section 2.3 .1 on page 47). The expected sinusoidal dependence is complicated by the $E^{4}$ synchrotron radiation loss. The longitudinal polarization of a beam with polarization $P_{\circ}$ at the source is expected to vary in the End Station A as

$$
P_{z}=P_{\circ} \cos \left(\pi\left(E+0.198(E / 48.362)^{4}\right) / 3.2374\right) .
$$


Data taken at different A-line energies were fitted with the expected $E$ (from the flip coil) dependence with the polarization magnitude $P_{\circ}$ and the energy offset $\Delta$ as fit parameters, as shown in figure 2.5. The flip coil reading was

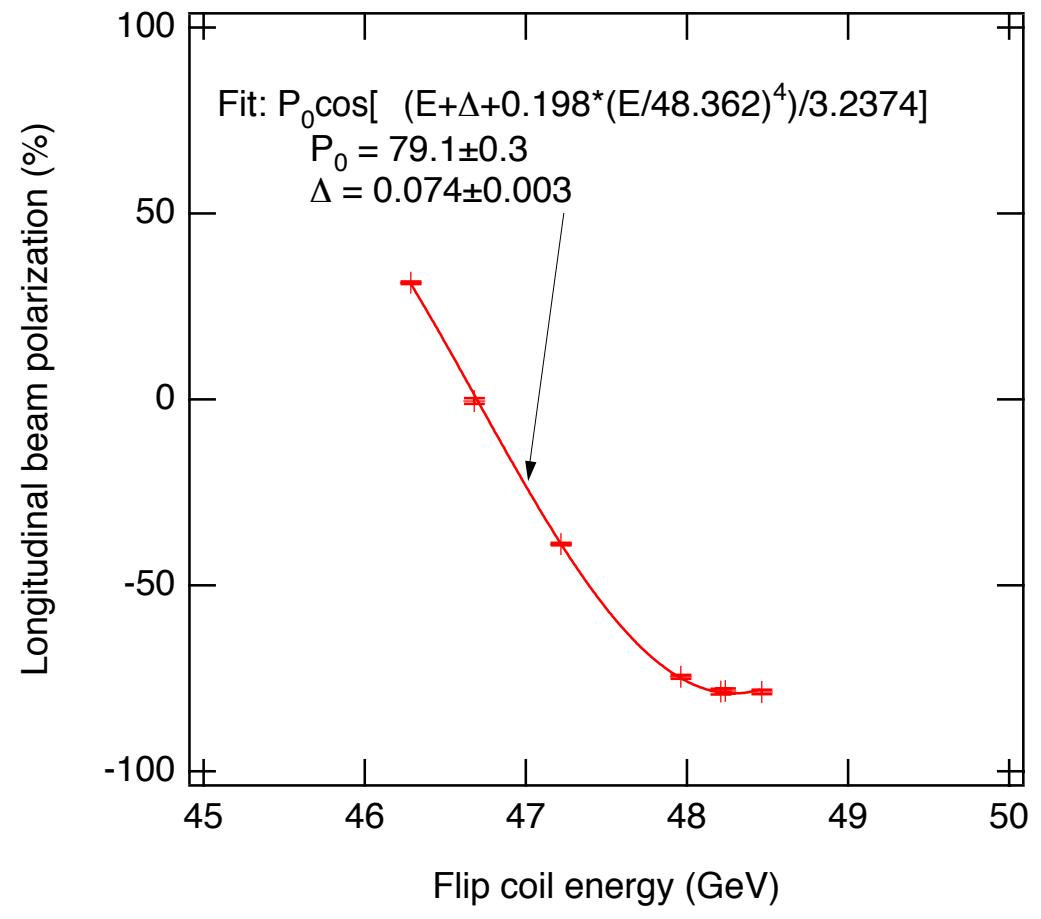

Figure 2.5: Measured beam polarization versus the nominal A-line beam energy as read out by a flip coil.

determined to be $74 \mathrm{MeV}$ below the true energy of $48.30 \mathrm{GeV}$ in the End Station A.

The polarimeter also established the absolute sign of the beam polarization in the End Station A. The Møller cross section is larger if the electron spin of the target and beam are anti-parallel. Knowing the sign of the asymmetry (negative) and the target polarization direction (the electron spin is 
opposite to the direction of electron magnetic moment, which is established by the direction of the current in the Helmholtz coils) the beam helicity orientation is extracted. The '01' polarization state was assigned to negative helicity in the End Station A and '10' to the positive one, in agreement with the conventions from section 2.3.5 (page 51).

\subsection{Polarized ${ }^{3} \mathrm{He}$ target}

The high density polarized ${ }^{3}$ He target was a source of polarized neutrons. It was developed from a similar target used in E142 experiment [82]. The target was a two-chambered $30 \mathrm{~cm}$ long and $2 \mathrm{~cm}$ in diameter glass tube

holding $2.6 \times 10^{20}$ atoms $/ \mathrm{cm}^{3}$ of ${ }^{3} \mathrm{He}$. The ${ }^{3} \mathrm{He}$ was polarized via spin exchange with optically pumped rubidium vapors and was kept aligned in 10-30 Gauss holding field. The average polarization was $38 \%$ for the experiment with a maximum of nearly $50 \%$ [83].

\subsubsection{Optical pumping and spin exchange}

The targets with a high density of ${ }^{3} \mathrm{He}$ are commonly polarized using the technique of optical pumping [84]. In this two step process the rubidium vapor is first optically polarized. Then the polarization of the rubidium electrons is transferred to the ${ }^{3} \mathrm{He}$ nucleus by the spin-exchange hyperfine interaction.

The circularly polarized laser light at $794.7 \mathrm{~nm}$ with positive, for example, helicity excites the $5 S_{1 / 2}(m=-1 / 2)$ ground state to the $5 P_{1 / 2}(m=+1 / 2)$ 
state in rubidium vapor (the D1 line). A simplified diagram of the process is shown in fig. 2.6. Radiative decays to the ground state favor the

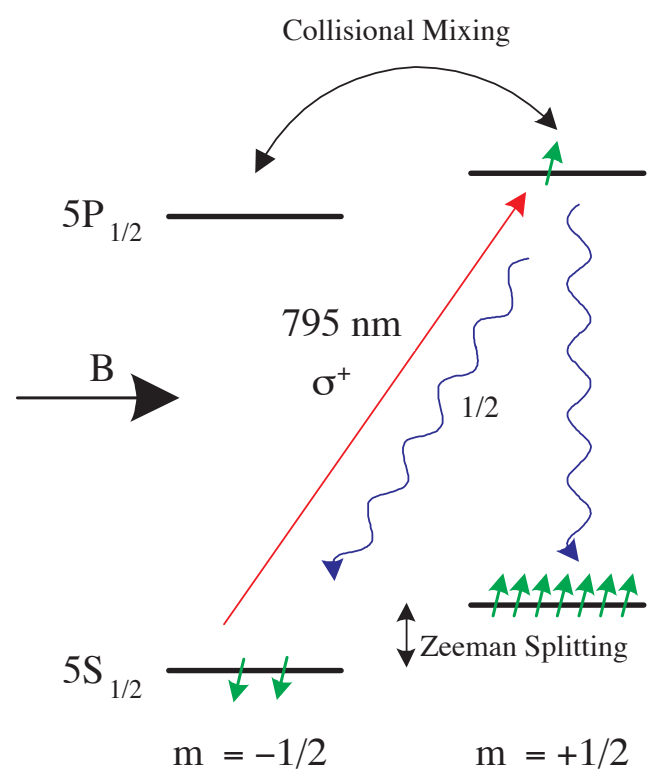

Figure 2.6: Optical pumping of Rubidium vapor by circularly polarized light.

$5 S_{1 / 2}(m=-1 / 2)$ state over $5 S_{1 / 2}(m=+1 / 2)$ state by a factor of two. Every three photons absorbed would increase the population of $5 S_{1 / 2}(m=+1 / 2)$ by one. By adding 65 torr of $\mathrm{N}_{2}$ the $5 P_{1 / 2}(m= \pm 1 / 2)$ are collision mixed at a rate exceeding the radiative decay. The relaxation into the $5 S_{1 / 2}$ ground state now occurs equally into the two magnetic substates. Only two photons are required to increase the population of $5 S_{1 / 2}(m=+1 / 2)$ by one. The polarization of the valence electrons of $\mathrm{Rb}$ competes with several depolarizing mechanisms. The most important are spin destruction from $\mathrm{Rb}-{ }^{3} \mathrm{He}$ and $\mathrm{Rb}-\mathrm{Rb}$ collisions (both dominate and contribute approximately equal 
amounts of relaxation), and interactions of $\mathrm{Rb}$ with walls of the target cell. The optical pumping rate of the optically thick $\mathrm{Rb}$ vapor $\left(10^{14}\right.$ atoms $\left./ \mathrm{cm}^{3}\right)$ with sufficient laser power is $1 / \Gamma_{\text {pump }} \approx 10^{-6}$ s and it dominates over the competing $1 / \Gamma_{\text {relax }} \approx 10^{-3} \mathrm{~s}$ spin relaxation rate. A Rb polarization of almost $100 \%$ could be achieved.

The spin exchange between the electrons of $\mathrm{Rb}$ and the nucleus of ${ }^{3} \mathrm{He}$ is mainly governed by the Fermi contact term in the hyperfine hamiltonian [85] which corresponds to the overlap of the $\mathrm{Rb}$ electron with the ${ }^{3} \mathrm{He}$ nucleus. The rate of this exchange, $\Gamma_{S E}$, is proportional to the relative velocity $v$ between the ${ }^{3} \mathrm{He}$ and $\mathrm{Rb}$, and to the number density of $\mathrm{Rb}, \mathrm{n}_{R b}$ :

$$
\Gamma_{S E}=\left\langle v \sigma_{S E}\right\rangle n_{R b}
$$

where the velocity averaged cross section is $1.2 \times 10^{-19} \mathrm{~cm}^{3} / \mathrm{s}[86]$. With $n_{R b} \approx 10^{14} \mathrm{~cm}^{-3}$ polarization times for ${ }^{3} \mathrm{He}$ are on the order of $1 / \Gamma_{S E} \approx$ $25-40$ hours. The nuclear polarization $P_{\left({ }^{3} \mathrm{He}\right)}$ of ${ }^{3} \mathrm{He}$ increases in time according to the following equation:

$$
P_{\left({ }^{3} H e\right)}(t)=P_{(R b)} \frac{\Gamma_{S E}}{\Gamma_{S E}+\Gamma_{R}}\left[1-e^{-\left(\Gamma_{S E}+\Gamma_{R}\right) t}\right],
$$

where $P_{(R b)}$ is the equilibrium polarization of $\mathrm{Rb}(\approx 100 \%)$ and $\Gamma_{R}$ is the total spin relaxation rate of ${ }^{3} \mathrm{He}$. The polarization curve of the best cell, Picard, is shown in fig. 2.7.

The biggest source of the ${ }^{3}$ He depolarization were the collisions between ${ }^{3}$ He atoms causing the exchange of nuclear spin and orbital angular momentum. For the cell Picard they resulted in $1 / \Gamma_{D}=84$ hours. Other, smaller 


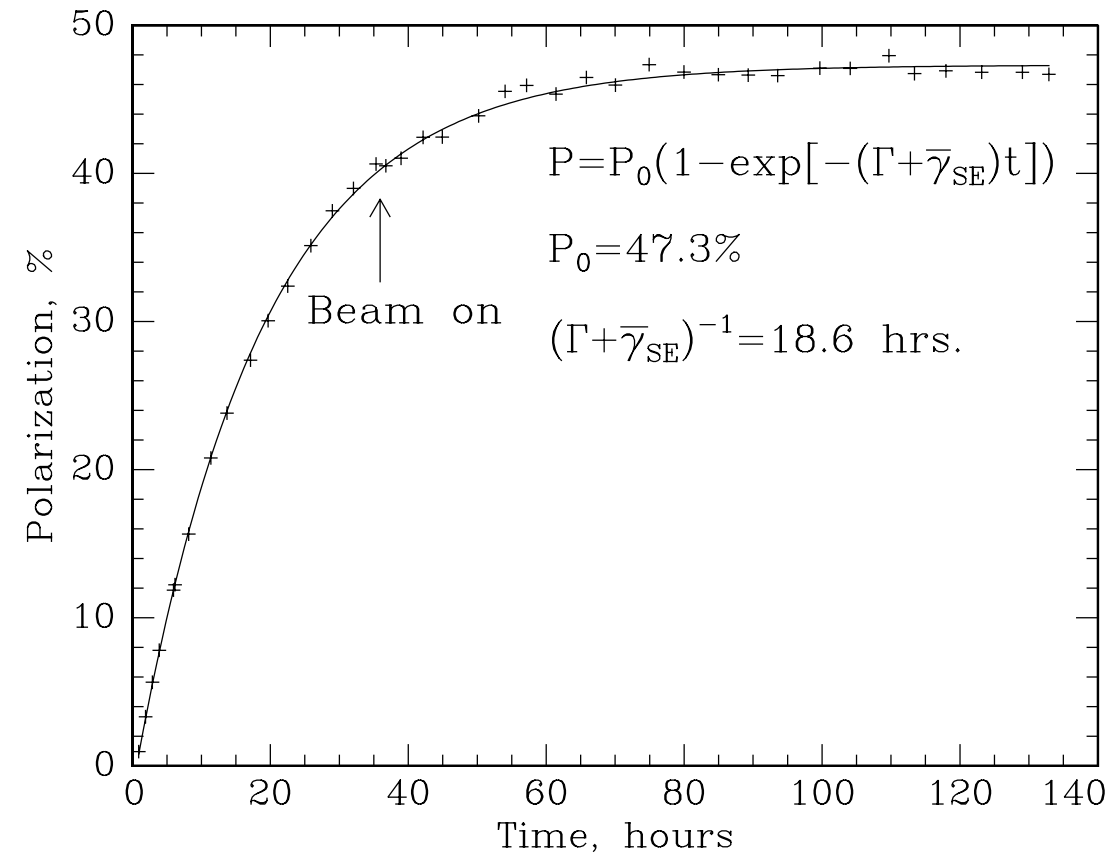

Figure 2.7: Polarization of the target cell Picard during the run.

contributions were caused by paramagnetic gaseous impurities embeded in the cell walls, gradients of the magnetic field perpendicular to the alignment field, and from the beam ionization of ${ }^{3} \mathrm{He}$. In our experiment the other sources resulted in $1 / \Gamma_{O} \equiv \sum_{i}\left(1 / \Gamma_{i}\right)>400$ hours. For the Picard cell the total relaxation was measured to be [83]

$$
1 / \Gamma_{R}=1 / \Gamma_{D}+1 / \Gamma_{O}=84 \pm 5 \mathrm{hrs},
$$

and a maximum polarization of $47.3 \%$ was achieved (see fig. 2.7 ). 


\subsubsection{Target cells}

The high polarization of the target that translates into long relaxation time is one of two goals of the target construction. The other is a large dilution factor $f$. Let us recall that $f$ is the ratio of the number of electrons scattered by the ${ }^{3}$ He to the total number of electrons scattered by the target (see sec. 3.10, page 154). Both goals are equally important because the running time for a given statistical error is inversely proportional to the square of the product of the polarization and the dilution. The dilution factor can be increased by increasing the density of ${ }^{3} \mathrm{He}$ or by decreasing the thickness of the cell windows traversed by the beam.

The target consisted of two chambers. The upper pumping cell had a 68-84 $\mathrm{cm}^{3}$ volume and contained ${ }^{3} \mathrm{He}$ and a few tens of $\mathrm{mg}$ of $\mathrm{Rb}$. It was connected with the target cell by a $10 \mathrm{~mm}$ diameter transfer tube. The cells

Table 2.3: Chemical Composition of Corning 1720 Glass by Weight.

\begin{tabular}{llllccccc}
\hline Compound & $\mathrm{SiO}_{2}$ & $\mathrm{Na}_{2} \mathrm{O}$ & $\mathrm{CaO}$ & $\mathrm{MgO}$ & $\mathrm{B}_{2} \mathrm{O}_{3}$ & $\mathrm{Al}_{2} \mathrm{O}_{3}$ & $\mathrm{~K}_{2} \mathrm{O}$ & $\mathrm{As}_{2} \mathrm{O}_{3}$ \\
\hline & 60.7 & 1.0 & 8.6 & 7.4 & 5.0 & 17.3 & 0.2 & 0.5 \\
& 57 & 1.0 & 5.5 & 12 & 4.0 & 20.5 & & \\
Ave: & 62 & 1 & 8 & 7 & 5 & 17 & & \\
\hline
\end{tabular}

were built from 1720 Corning glass, primarily because of its low permeability to ${ }^{3}$ He. The chemical composition of the glass coming from three different sources is given in table 2.3 [87]. The new concave windows for the target cells were designed for our experiment. The windows had half of the thickness 
of the E142 convex design. At the same time they were able to withstand higher ${ }^{3}$ He pressures, since glass is stronger under compression than under tension. However, they could not sustain the high beam intensity. Six target cells and one reference cell broke after less than a week of operation in the beam. The exact cause of the breaking remains unknown. The targets and some of their parameters are listed in table 2.4 .

The thickness of the target cell windows was measured by three different methods: mechanically with a micrometer, by X-ray absorption with a ${ }^{55} \mathrm{Fe}$ and by using the interference between laser light reflections from the two surfaces of the window. The optical methods were the most precise, and their uncertainty of $3 \%$ was limited by the non-uniformity of the windows. The mechanical method, abandoned because it scratched or broke some windows, was accurate to $5 \%$ (the windows of cell Riker were measured mechanically only, for all others one or both optical methods were used).

The ${ }^{3}$ He density was measured by two methods. The first relied on measurement of the amount of ${ }^{3}$ He used for filling the cell. Then, after the cell was sealed, its density was determined by the broadening and shift of the Rb absorption lines by ${ }^{3}$ He. This way all the surviving cells could be re-measured after the run. The uncertainties of the ${ }^{3}$ He density measurements were $1.5 \%$. The $\mathrm{N}_{2}$ densities were measured during the filling process and were known with $5 \%$ uncertainty. 


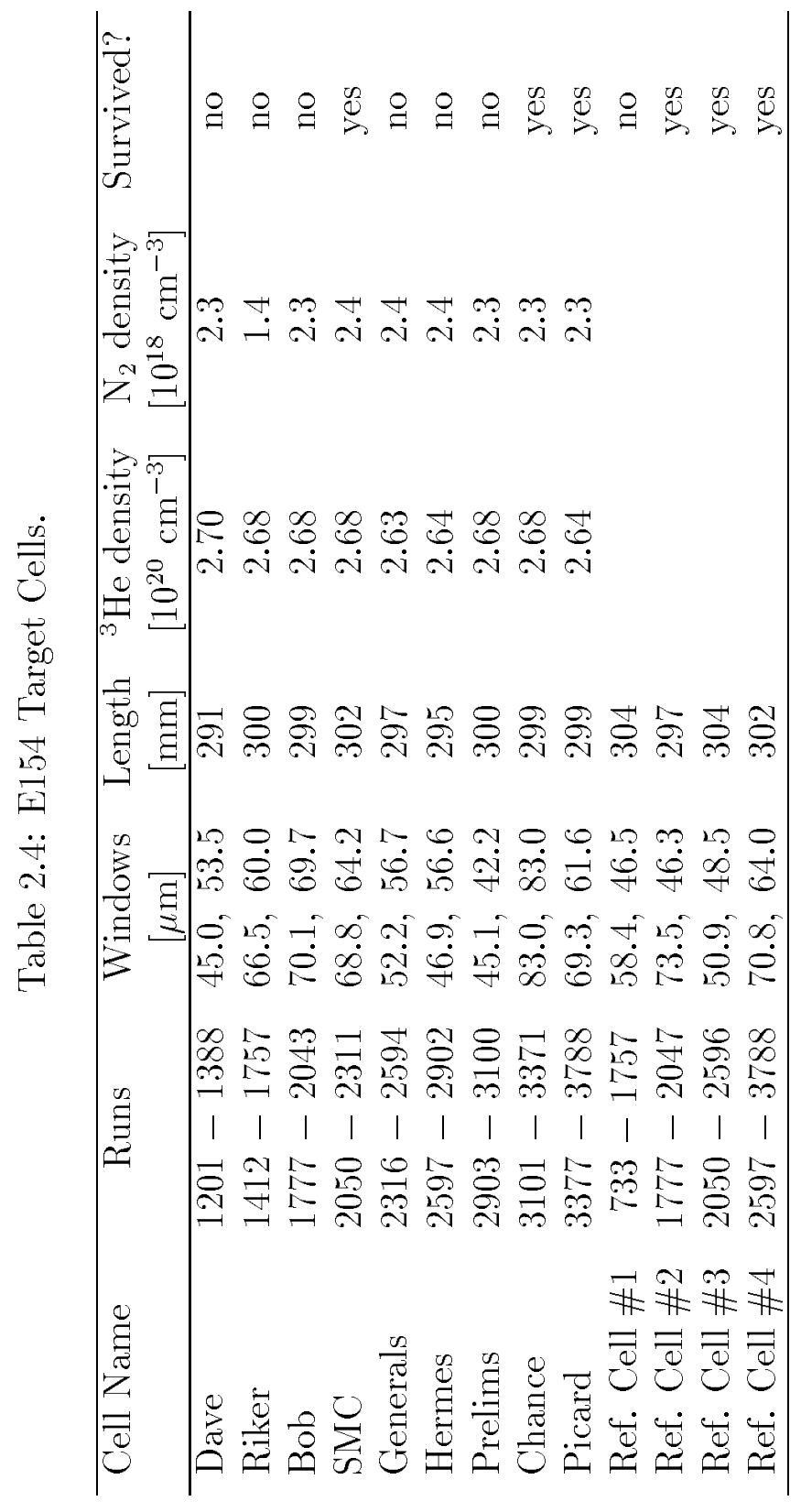




\subsubsection{Target setup}

The pumping chamber of the target was enclosed in a plastic oven and heated to $170-200{ }^{\circ} \mathrm{C}$. That vaporized the $\mathrm{Rb}$ to a density of about $10^{14}$ atoms $/ \mathrm{cm}^{3}$. The $\mathrm{Rb}$ was polarized by the lasers located in a specially constructed laser hut near the target. The light from each laser passed through a set of optical components, including a quarter-wave plate, to produce circularly polarized light, before reaching the pumping cell. There were four Argon Ion pumped Ti:Saphire lasers with $20 \mathrm{~W}$ total power, and three fiber-coupled diode arrays, each with about 15-17 W of power, continuously polarizing Rb. The ${ }^{3} \mathrm{He}$, polarized via spin exchange with the $\mathrm{Rb}$ in the upper cell, diffused through a $60 \mathrm{~mm}$ long and $10 \mathrm{~mm}$ in diameter transfer cell to the lower target cell with a time constant of about 10 minutes. The schematic of the target apparatus is shown in fig. 2.8. The endcaps of the cell were cooled by ${ }^{4}$ He jets to relieve the heating caused by the beam. The temperatures of the target were monitored by seven Resistive Thermal Devices (RTDs) mounted in various places, five on the target and two on the pumping cells. The temperature of the target remained at $60-80^{\circ} \mathrm{C}$. The residual $\mathrm{Rb}$ density was on the order of $10^{11}$ atoms $/ \mathrm{cm}^{3}$, negligible in comparison to the primary target components: $2.6 \times 10^{20}$ atoms $/ \mathrm{cm}^{3}$ of ${ }^{3} \mathrm{He}$ and $2.4 \times 10^{18}$ molecules $/ \mathrm{cm}^{3}$ of $\mathrm{N}_{2}$. The target was mounted in a movable holder and could be placed in the beam with a sub-millimeter accuracy. The holder had another position with a reference cell placed below the target. This cell could be filled remotely

with a variable pressure of ${ }^{3} \mathrm{He}$ and was used for dilution factor studies. The 


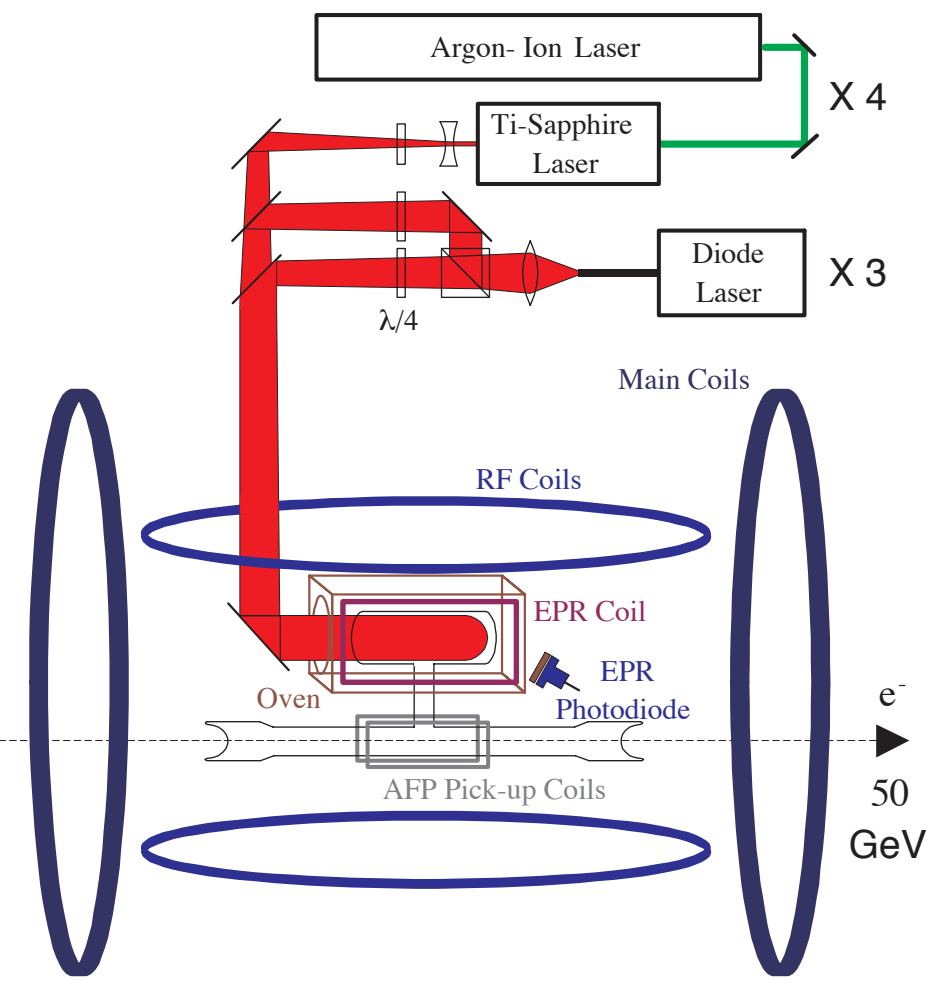

Figure 2.8: Schematic of the E154 target.

whole target assembly was enclosed in the scattering chamber kept under a few mtorr of vacuum. Outside of the scattering chamber were two $1.4 \mathrm{~m}$ diameter Helmholtz coils. They produced a 10-30 G holding field to align the nuclear spins of ${ }^{3}$ He along the beam direction. A similar set of coils was used to produce a holding field perpendicular to the beam direction used for the runs with transverse target polarization for the measurement of $A_{\perp}$. There were also other sets of coils for polarization measurements. 


\subsubsection{Polarimetry and polarization results}

The target polarization was measured by two independent methods [88]. One was the NMR technique using Adiabatic Fast Passage (AFP). The other used the shift of the $\mathrm{Rb}$ Zeeman resonance (EPR) frequency due to the ${ }^{3} \mathrm{He}$ polarization.

The AFP-NMR was used regularly during the run. A set of $42.75 \mathrm{~cm} \mathrm{di-}$ ameter Helmholtz drive coils above and below the target provided a $72 \mathrm{mG}$ RF field at $92.0 \mathrm{kHz}$, while the main holding field was swept from 18 to $36 \mathrm{G}$, through the Larmor resonance at $28.4 \mathrm{G}$. The sweep rate of $1.2 \mathrm{G} / \mathrm{s}$ was slow enough for the nuclear spins to follow the changing field, but fast enough to avoid the de-phasing of the spins while passing through the resonance. The resulting nuclear spin flip induced a signal in a set of 200 turn copper pick-up coils, wound on a $76.2 \mathrm{~mm}$ by $25.4 \mathrm{~mm}$ Teflon form and centered around the target cell. The coils connected with a $100 \mathrm{pF}$ capacitor formed an $\mathrm{LC}$ resonant circuit. The signal was pre-amplified and the $92 \mathrm{kHz}$ modulation was subtracted by the lock-in amplifier. The resulting NMR signal, see fig. 2.9, was proportional to the ${ }^{3}$ He polarization. The proportionality constant is determined from the thermal equilibrium Boltzman polarization $P_{p}$ of protons in a water sample,

$$
P_{p}=\tanh \left(\frac{\mu_{p} B}{k_{B} T}\right),
$$

where $\mathrm{B}$ equal $21.61 \mathrm{G}$ for a proton $92 \mathrm{kHz}$ resonance. The proton signals were a few $\mu \mathrm{V}$, in comparison with $200 \mathrm{mV}$ of ${ }^{3} \mathrm{He}$. Many sweeps were

needed to form an average. The typical water signal is shown in fig. 2.9. The 
${ }^{3} \mathrm{He}$ AFP Signal
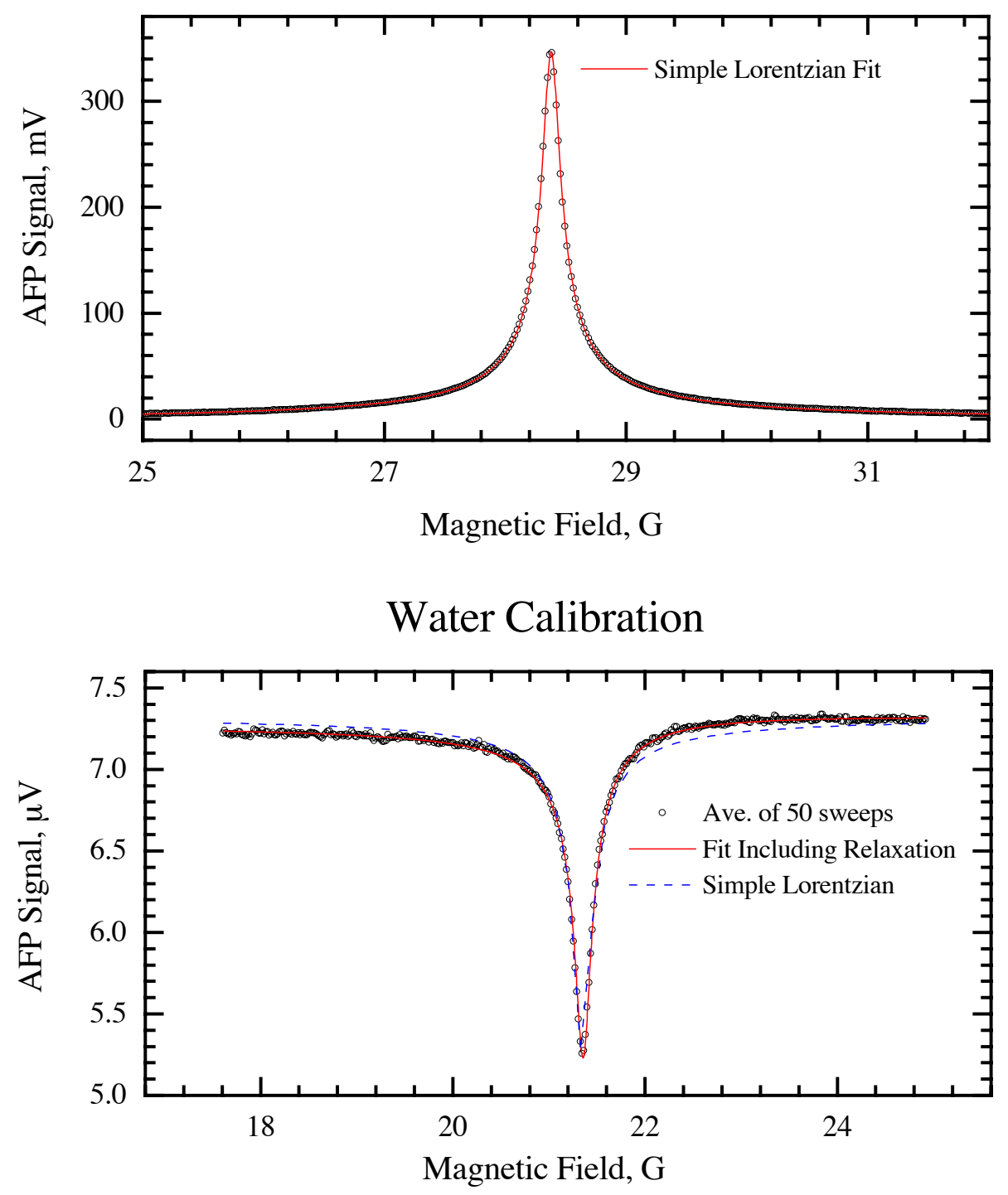

Figure 2.9: AFP-NMR signals of ${ }^{3} \mathrm{He}$ and water. 
uncertainty in the height of that signal $(1.8 \%)$ together with the uncertainty of the normalized ${ }^{3}$ He density $(1.6 \%)$ and the computed coil flux ratio (1.6\%) were the dominant contributions to the total of $3.4 \%$ uncertainty [83] of the AFP-NMR polarization measurement.

The second method, the EPR polarimetry, was used to calibrate the AFP system. It is based on the shift of the rubidium Zeeman resonance due to the magnetic field created by the polarized ${ }^{3} \mathrm{He}$. There are two contributions to the EPR resonance shift. The first one is proportional to the ${ }^{3} \mathrm{He}$ polarization coming from the polarization transfer in the $\mathrm{Rb}-{ }^{3} \mathrm{He}$ spin exchange interaction. The other is due to the classical magnetic field produced by the ${ }^{3} \mathrm{He}$ magnetization. The shifts are substantial, about $20 \mathrm{kHz}$ out of 8 $\mathrm{MHz}$, and easy to measure. By taking the difference in the EPR frequency between two measurements with opposite ${ }^{3} \mathrm{He}$ polarization we could isolate the part proportional to the ${ }^{3} \mathrm{He}$ polarization. The resonance was observed by monitoring the intensity of the fluorescence photons of the $\mathrm{D}_{2} \mathrm{Rb}$ line as a function of the RF frequency [88]. In the highly polarized $\mathrm{Rb}$ vapor the atoms are in the $F=3, m=3$ state (or $m=-3$ for the opposite polarization). The RF field at the EPR frequency corresponding to $m$ transition $3 \rightarrow 2$ increases the $m=2$ population capable of absorbing the laser light, and thus increases the fluorescence intensity. The typical EPR frequency measurement is shown in fig. 2.10. For the precise determination of the EPR frequency the magnetic field was measured by the Flux-Gate magnetometer and was kept stable at the $10^{-5}$ level. That enabled the frequency shift to be 


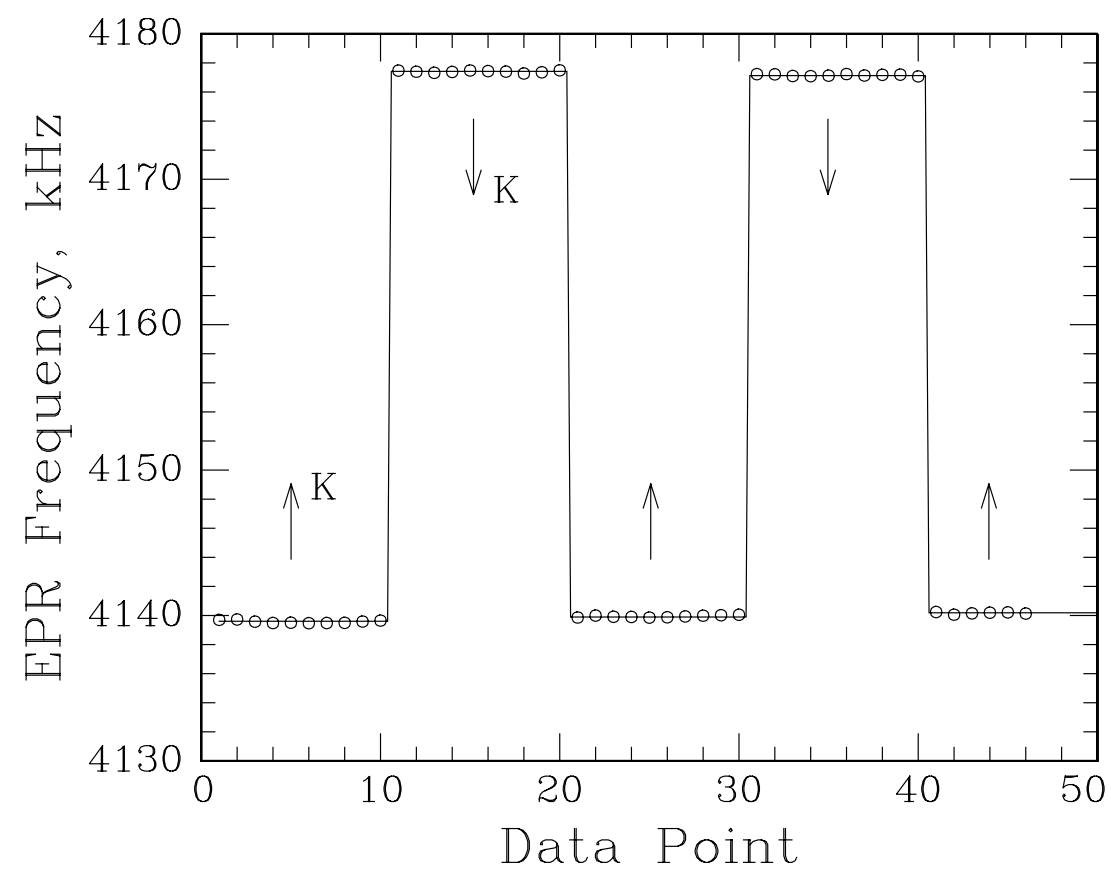

Figure 2.10: EPR frequency measurement for both polarization directions (denoted by arrows) for the target cell Picard.

measured with $0.5 \%$ accuracy. The proportionality constant, $\kappa_{\circ}$, between the frequency shift and the ${ }^{3}$ He polarization was measured [88] within $1 \%$. Other uncertainties include the polarization gradient (1.5\%) needed to connect the EPR measured polarization in the pumping cell with the AFP measurement in the target cell, the gas density (1.5\%) and the magnetic field shift (1.3\%). The total systematic error of the EPR method is $3.0 \%$.

The AFP and EPR polarization measurements disagree by $1.2 \sigma$ (combined in quadrature). Assuming Gaussian distributions for the errors, that corresponds to $23 \%$ probability of that or a larger difference. We do not exclude the possibility of unknown systematic effects. Thus, rather that taking 
the average of the results, we conservatively increase the systematic uncertainty so it covers both measurements with their errors. We also include the total systematic drift of the AFP coils calibration constants, equal to $1.7 \%$, of measurements before and after the runs. The final target polarization uncertainty is $4.8 \%$.

\subsubsection{Target polarization direction}

The direction of the ${ }^{3} \mathrm{He}$ polarization with respect to the beam direction was determined from the orientation of the holding magnetic field with respect to the beam direction and the orientation of the magnetic field with respect to the polarization.

The direction of the magnetic field was determined in four ways: 1. using a compass. 2. Next the sign of the field was measured with the Flux-gate magnetometer. 3. Then with a Hall probe calibrated by the spectrometer magnets. 4. Finally, knowledge of the direction of the coil winding and the electric current also established the magnetic field orientation. All methods gave the same result.

The relative orientation of the polarization to the magnetic field was established in three ways. The first method uses the sign of the AFP signals. The magnetic moments of the protons and ${ }^{3}$ He have opposite signs. If their spins are initially aligned, then the sweep with the same sign of $\mathrm{dB} / \mathrm{dt}$ will produce the opposite signs of the NMR signal. That relates the direction of the ${ }^{3}$ He spin to that of protons, which are aligned with the magnetic field. The second method established the direction of the ${ }^{3}$ He magnetization due to 
polarization and thus the direction of the spin from the sign of the EPR shift. Finally, the observation of the masing effect resulting in the depolarization due to the coupling of the ${ }^{3}$ He spins to the pickup coils gives the polarization orientation. All the methods were consistent.

\subsection{Magnetic spectrometers}

The new magnetic spectrometers were designed for the E154 and the following E155 experiments at End Station A [89, 90] with the $50 \mathrm{GeV}$ electron beam. The goal was to obtain the highest possible counting rates consistent with the ability to cleanly identify and determine the energy of the scattered electrons in the presence of a large background of pions and low energy neutral particles. An important factor was the short SLED beam pulse width of about $240 \mathrm{~ns}$ resulting in 10 times higher instantaneous rates (with the same electrons per spill) in comparison with the previous ESA experiments, E142 and E143.

The system consisted of two independent magnetic spectrometers centered at $2.75^{\circ}$ and $5.5^{\circ}$ with respect to the incident $48.3 \mathrm{GeV}$ electron beam. That allowed for the kinematic range of $0.014 \leq x \leq 0.8$ in the Bjorken $x$ and $1 \mathrm{GeV}^{2} \leq \mathrm{Q}^{2} \leq 17 \mathrm{GeV}^{2}$ in the momentum transfer squared. The fixed angles correspond to $x$ and $Q^{2}$ values shown in fig. 2.11. The lowest $x$ value, corresponding to the lowest momentum of the scattered electrons, was determined by the tolerable level of the hadronic background and the requirement $y=\left(E-E^{\prime}\right) / E \leq 0.8$ dictated by the rapid increase of radiative corrections 


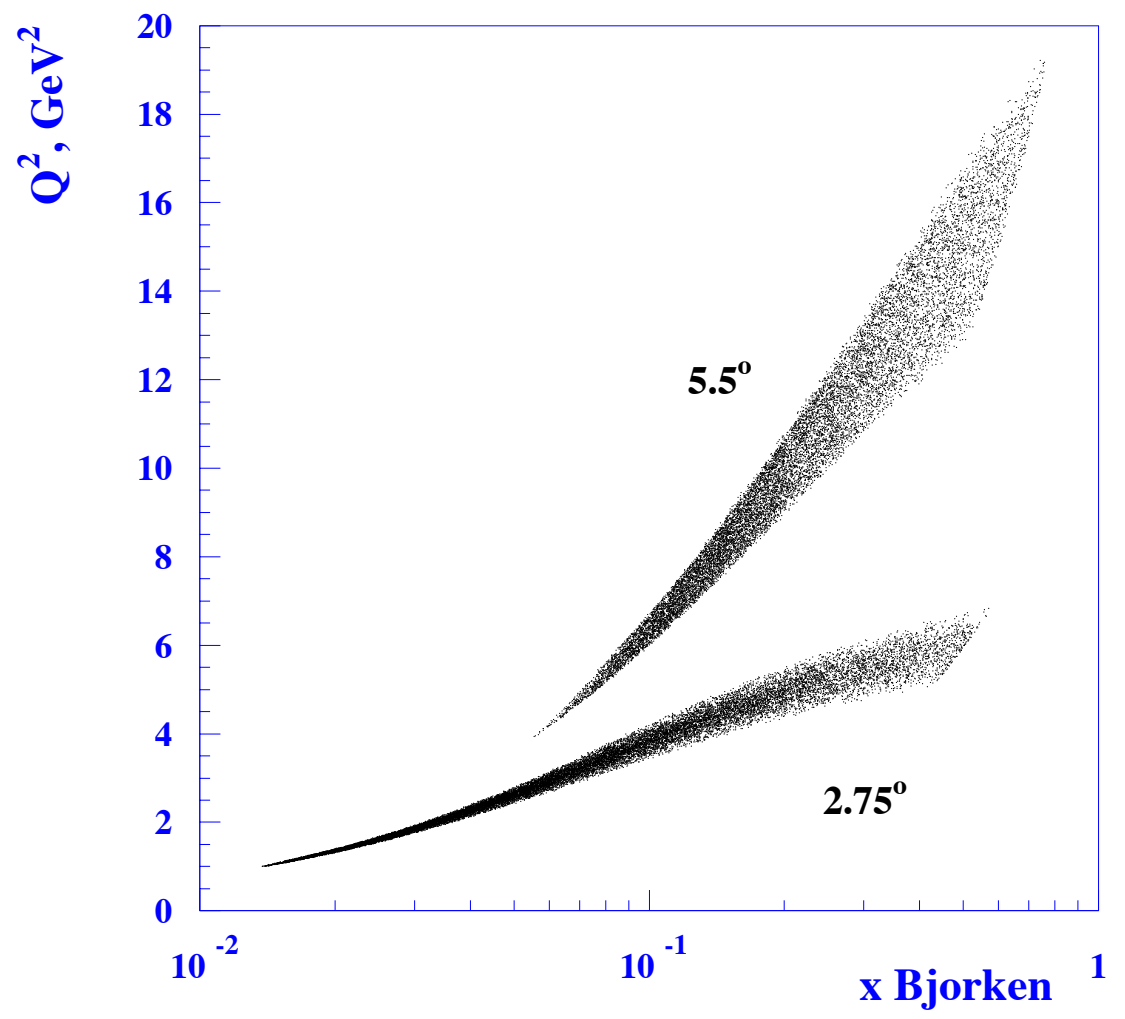

Figure 2.11: E154 kinematic coverage in $x$ and $Q^{2}$.

at high $y$. The highest $x$ value (the highest momentum) was set by the deep inelastic condition $W^{2} \geq 8 \mathrm{GeV}^{2}$, to be far away from the resonances. The calculated unpolarized differential cross section as a function of the scattered momentum is shown in fig. 2.12. The measured momentum of the scattered electrons ranged from $10 \mathrm{GeV}$ up to $44 \mathrm{GeV}$ in $2.75^{\circ}$ and up to $39 \mathrm{GeV}$ in $5.5^{\circ}$.

The conceptual design of the spectrometers is straightforward. The electrons scattered off the target pass through a set of two dipole magnets in a vertical S-band configuration. The $2.75^{\circ}$ arm has an additional quadrupole, 


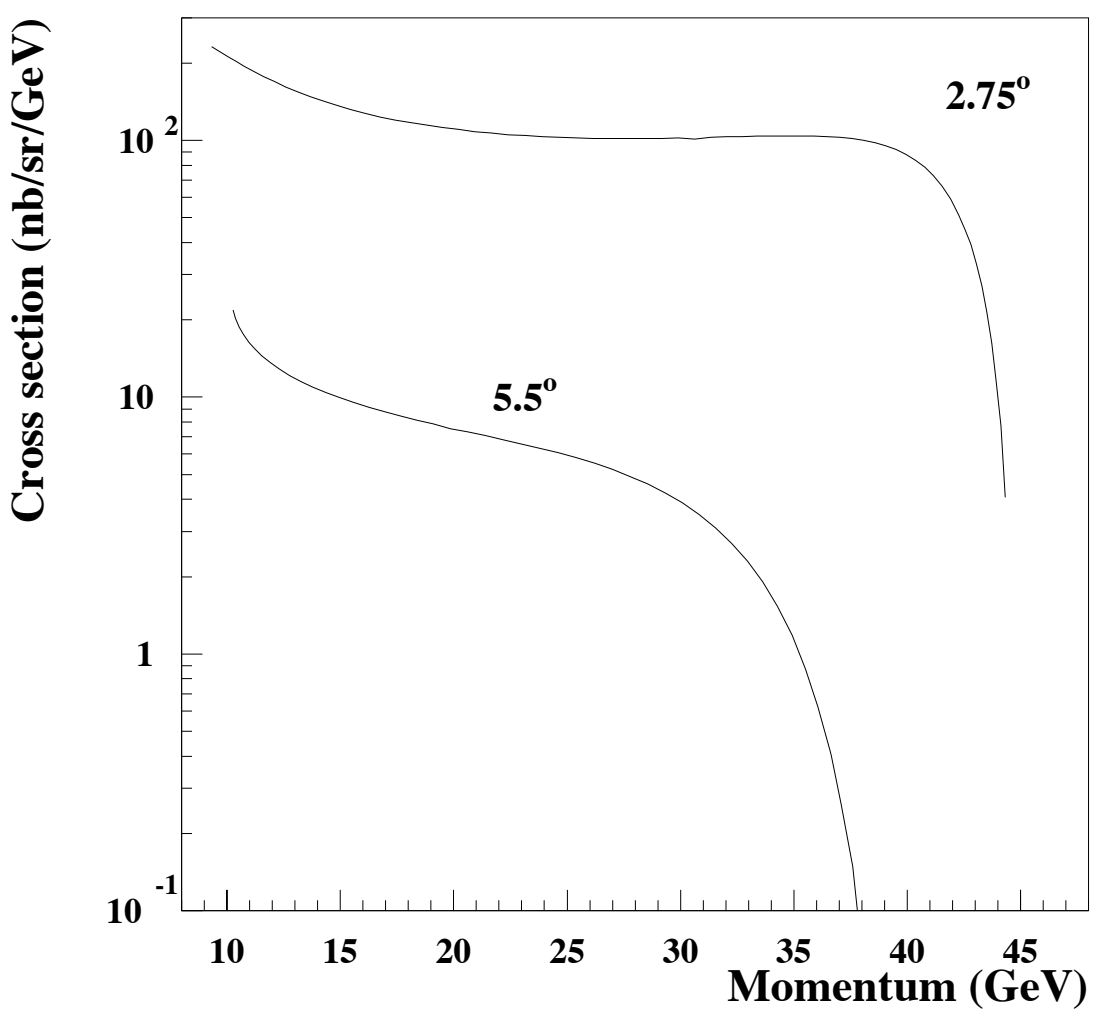

Figure 2.12: Differential cross section for the ${ }^{3}$ He target (per nucleon).

positioned between the dipoles, which defocussed particles in the horizontal direction reducing the instantaneous rates per detector element. Next the electrons leave signals in two threshold Cherenkov counters and fire some of the scintillator hodoscope fingers. Finally they are absorbed by the electromagnetic lead glass calorimeter in a fly's eye configuration. The schematic of the spectrometer elements is shown in fig. 2.13. 

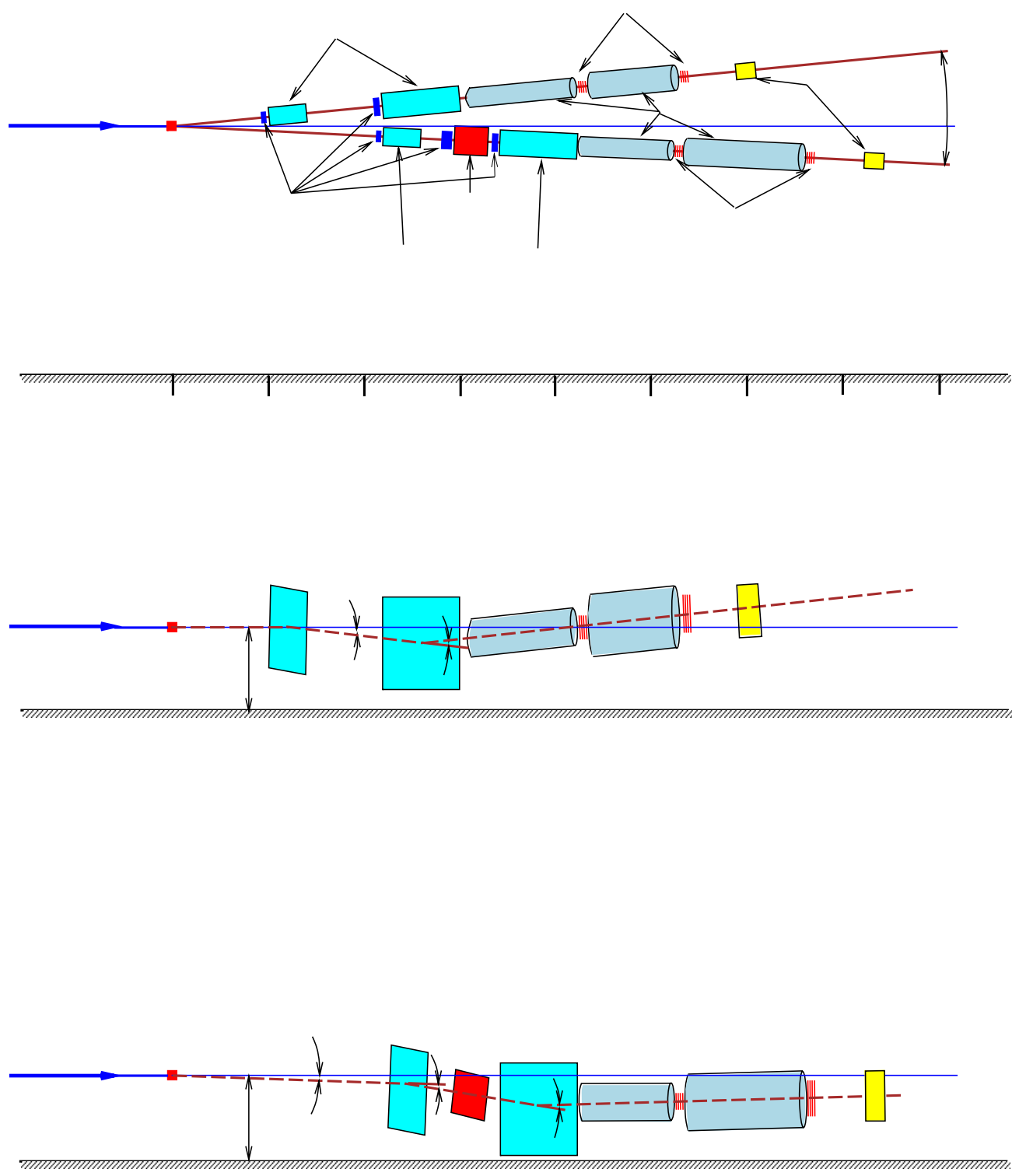

Figure 2.13: Schematic layout of the E154 spectrometers.

The optics of the (vertical) S-band configuration satisfied the main performance requirements. It allowed a large solid angle over a broad range of 
momenta. A solid angle of $0.3-0.5 \mathrm{msr}$ for $10-40 \mathrm{GeV}$ electrons was achieved for the $5.5^{\circ}$ spectrometer [91], and a variable solid angle from 0.05 to $0.1 \mathrm{msr}$ for 9-40 GeV electrons in the $2.75^{\circ}$ arm. Furthermore, an optimized choice of bending strengths and detector geometry assured a two-bounce system: the neutral particles (like photons) from the target had to bounce at least twice to enter the detectors. That supressed the neutral background to a tolerable level. Figure 2.14 shows the optics of the spectrometers. The central trajectory of the spectrometers corresponded to $20 \mathrm{GeV}$ scattered momentum. It was first bent downwards by $3.7^{\circ}$, then upwards by the rear dipoles. The quadrupole in the $2.75^{\circ}$ spectrometer defocussed electrons in the horizontal plane and reduced the instantaneous rates per detector element. It also focused the trajectories (at $p=18 \mathrm{GeV}$ at the shower) in the vertical (bend) plane resulting in spread of low momentum particles at the shower counter, an important element in reducing the probability of electron-pion overlaps.

The magnet currents were stable to $0.1 \%$ level. They were monitored several times per run. The NMR probes were used in each dipole and a Hall probe in the quadrupole to check the field every shift.

The acceptance of the spectrometers was defined by the set of collimators [91] and matched the active areas of the detectors.

All the above considerations and the momentum reconstruction relied entirely on the Monte Carlo simulation that used the measured magnetic fields and the precision alignment data of the spectrometers elements. Also the shower counters were calibrated with respect to the momentum and not 

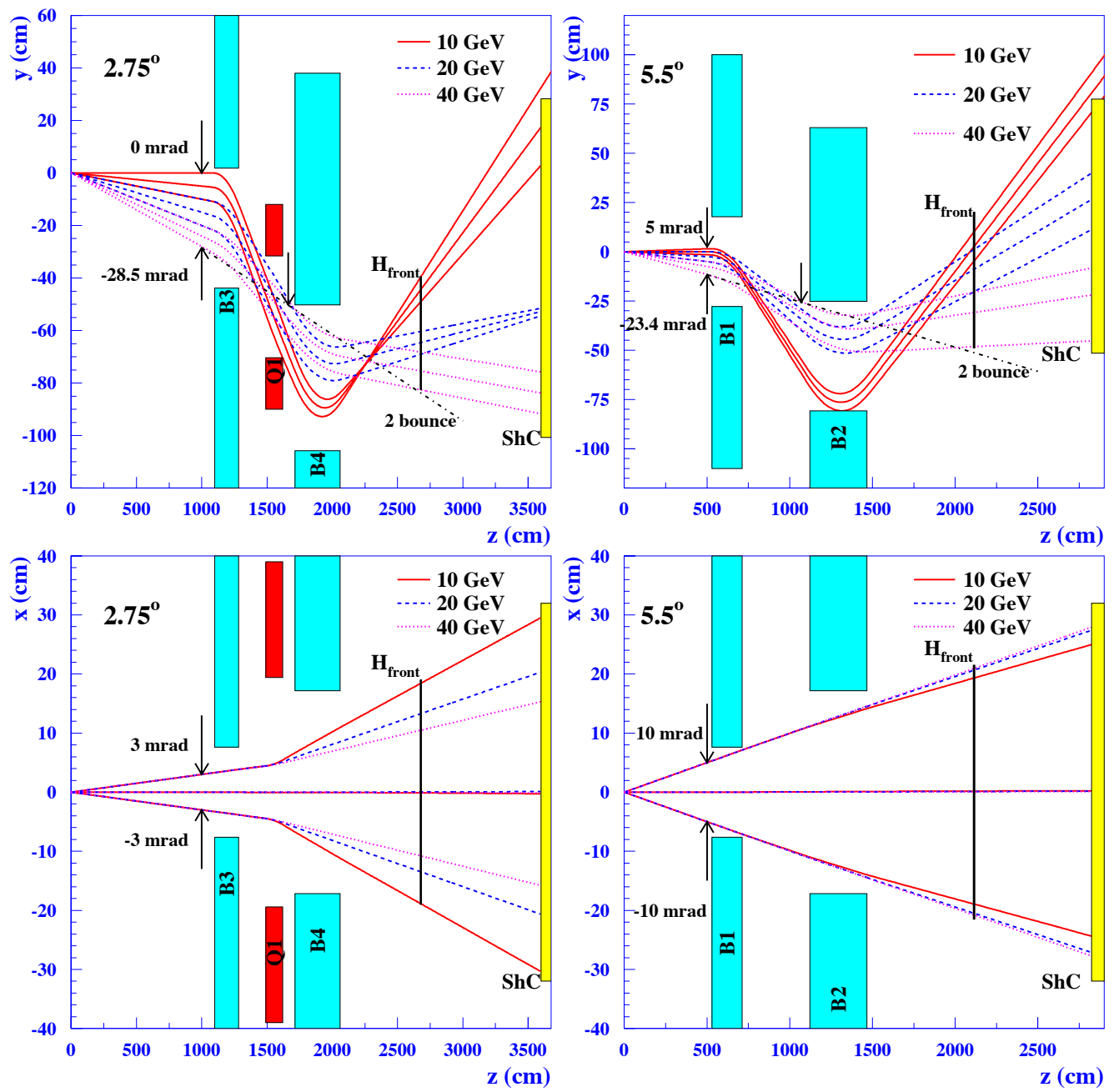

Figure 2.14: Optics of the $2.75^{\circ}$ (left) and $5.5^{\circ}$ (right) spectrometers in the vertical (top) and horizontal (bottom) planes.

in an independent way. It was thus important to check the integrity of the optics model with some special runs. Two test were performed [91].

One of the tests used a "sieve slit" (a tungsten mask with small holes) in front of the spectrometers, so that the angles of the scattered electrons 
were well defined and could be checked against the reconstructed values. The agreement was quite good.

In the second test the elastic peak was observed with $\approx 8 \mathrm{GeV}$ electrons and adjusted values of the spectrometers central momentum. The peak position, or the shape of the end point cross-section if the peak is not visible, directly determines the momentum. The results of the analysis results were [91]: $E=8.16 \pm 0.16 \mathrm{GeV}\left(2.75^{\circ}\right)$ and $E=7.95 \pm 0.16 \mathrm{GeV}\left(5.5^{\circ}\right)$ in

agreement with the flip coil beam energy $E=8.095 \mathrm{GeV}$. The errors were dominated by systematics. As a result of this analysis we included a $2 \%$ error on the energy of the scattered electrons in the systematic error on $g_{1}^{n}$.

\subsection{Cherenkov detectors}

The precision inclusive measurement of the cross section asymmetries requires a good separation of electrons from the hadronic background, mainly pions, created at the target. The basic tools for the electron identification and pion rejection were threshold Cherenkov counters, two per spectrometer. Filled with the $\mathrm{N}_{2}$ at sub-atmospheric pressures they were set at $19 \mathrm{GeV}$ $\left(2.75^{\circ}\right)$ and $16 \mathrm{GeV}\left(5.5^{\circ}\right)$ pion threshold momenta. All the Cherenkov detectors worked under the same principle. The passing electrons produced a Cherenkov radiation which was reflected by mirrors to a wave-length shifter coated photomultiplier tube located outside of the envelope of spectrometer particles. The existing E142/E143 counters [92] were modified for E154. Many parts were reused. 


\subsubsection{Cherenkov radiation}

Cherenkov radiation occurs if a charged particle moves in a given medium with a velocity greater than the velocity of light. The angle $\theta$ of the emision of Cherenkov photons with respect to the particle trajectory with velocity $\beta$ in a medium having index of refraction $n$ is

$$
\cos \theta=\frac{1}{\beta n}
$$

For a given index $n$ the velocity $\beta$ for which the radiation occurs is established by the requirement that the angle $\theta$ is real, i.e. $\cos \theta<1$. The number of Cherenkov photons produced by an electron, per unit distance $x$ and unit wavelength $\lambda$ is given by

$$
\frac{d^{2} N_{\gamma}}{d x d \lambda}=\frac{2 \pi \alpha}{\lambda^{2}} \sin ^{2} \theta
$$

Let us now choose some arbitrary pion momentum thereshold, $p_{t}$. The corresponding velocity, $\beta_{t}=p_{t} / E_{t}=p_{t} / \sqrt{m_{\pi}^{2}+p_{t}^{2}}$, determines the index of refraction,

$$
\cos \theta_{t} \equiv 1=\frac{1}{\beta_{t} n} \Rightarrow n^{2}=1+\left(\frac{m_{\pi}}{p_{t}}\right)^{2},
$$

and thus, for ultra-relativistic electrons $(\beta \approx 1)$,

$$
\frac{d^{2} N_{\gamma}}{d x d \lambda}=\frac{2 \pi \alpha}{\lambda^{2}}\left(\frac{m_{\pi}^{2}}{p_{t}^{2}+m_{\pi}^{2}}\right)
$$

According to the above formula, the higher the pion momentum $p_{t}$ threshold, the smaller the number of Cherenkov photons, which is directly related to the number of photo-electrons produced at the cathode of the photomultiplier. 
That means a lower efficiency of the electron detection. On the other hand, the higher the threshold, the greater the pion rejection, since the ratio of pions to electrons drops rapidly as a function of the pion momentum [93]. One has to optimize between these two conflicting requirements. We chose the pion to electron ratio $\sim 4$ at the threshold $p_{t}$ which resulted in $p_{t}=19 \mathrm{GeV}$ at $2.75^{\circ}$ and $p_{t}=16 \mathrm{GeV}$ at $5.5^{\circ}$. The expected electron efficiency above one photoelectron signal exceeded $95 \%$. The pions with a velocity $\beta$ corresponding to momentum $p$ higher than the $p_{t}$ threshold produce Cherenkov radiation at the angle

$$
\cos \theta=\frac{\beta_{t}}{\beta},
$$

and thus create the number of photons,

$$
\left(\frac{d^{2} N_{\gamma}}{d x d \lambda}\right)^{\pi}=\left(\frac{d^{2} N_{\gamma}}{d x d \lambda}\right)^{e}\left[1-\left(\frac{p_{t}}{p}\right)^{2}\right],
$$

resulting in $3 / 4$ of the electron signal at $p=2 p_{t}$, where the cross section for the pion production is negligible with respect to the electron DIS cross section.

\subsubsection{Detector construction details}

The index of refraction $n$ for a chosen $p_{t}$ differs from 1 at the level of $10^{-5}$, see eq. (2.21). The easiest way to achieve such indices is to use a gasous radiator. We chose to use nitrogen for several reasons. The Cherenkov spectrum is inversly proportional to the square of the wavelength of the emitted photon, as seen from eq. (2.20). Most of the radiation comes from the shorter wavelengths. The $\mathrm{N}_{2}$ has excellent transmission from $150 \mathrm{~nm}$ to 
$700 \mathrm{~nm}$. In comparison, $\mathrm{O}_{2}$ or $\mathrm{CO}_{2}$ strongly absorb photons with wavelengths smaller than $190 \mathrm{~nm}$. In addition, nitrogen has a low $\delta$ production rate, a tolerable scintillation rate and is readily available at high purity. The index of refraction is determined by its density and thus by pressure at a given temperature. It is inferred from the Lorenz-Lorentz relation,

$$
\frac{n(\lambda)^{2}-1}{n(\lambda)^{2}+2}=K(\lambda) \rho
$$

where for $\mathrm{N}_{2}$ the coefficient $K(\lambda=546 \mathrm{~nm}) \approx 0.163 \mathrm{~cm}^{3} / \mathrm{g}$ [94]. The fact that $K$ varies with $\lambda$ introduces a mild $\lambda$ (between $100-600 \mathrm{~nm}$ ) dependence of $n$, with larger $n$ at smaller $\lambda$ 's. That gives a smooth rather than sharp pion threshold.

The importance of the length of the detector can be seen from eq. (2.22). When integrated over the distance $d x$, it shows that the number of Cherenkov photons is directly proportional to the length of the tank. We extended the existing tanks as much as allowed by the size of the detector huts, up to $6 \mathrm{~m}$. The dimensions and other parameters are listed in table 2.5. The tanks were cut in two and welded to a cylindrical insertion that had 1.27 $\mathrm{cm}$ thick walls. The insertions were ground and the entire interiors were treated with a mild acid etch and steam-cleaned to remove the aluminum oxide and other surface contaminants and then washed with alcohol to help evaporate the water remnants. The tanks were closed with thin aluminum end windows. The windows (from E142) for the larger diameter tanks were hydroformed to a concave shape that reduced the stress on them and thus could be made thinner (an important factor for minimizing $\delta$ production 
Table 2.5: The Cherenkov Detector Characteristics.

\begin{tabular}{lcccc}
\hline & $2.75^{\circ}$ & Spectrometer & $5.5^{\circ}$ & Spectrometer \\
Parameter & $2 \mathrm{C} 1$ & $2 \mathrm{C} 2$ & $5 \mathrm{C} 1$ & $5 \mathrm{C} 2$ \\
\hline Physical length $(\mathrm{m})$ & 5.6 & 6.5 & 5.8 & 4.3 \\
Radiator length $(\mathrm{m})$ & 5.3 & 6.1 & 5.6 & 4.0 \\
Interior radius $(\mathrm{cm})$ & 52.7 & 80 & 52.7 & 80 \\
Window thickness $(\mathrm{cm})$ & 0.15 & 0.1 & 0.15 & 0.1 \\
No. of mirrors & 2 & 3 & 2 & 3 \\
Mirror size $[x(\mathrm{~cm}) \times y(\mathrm{~cm})]$ & $51 \times 39$ & $71 \times 44$ & $51 \times 39$ & $71 \times 44$ \\
Mirror Radius $(\mathrm{cm})$ & 120 & 163 & 120 & 163 \\
Pion treshold $p_{t}(\mathrm{GeV})$ & 19 & 19 & 16 & 16 \\
$\mathrm{~N}_{2}$ pressure at $22^{\circ} \mathrm{C}(\mathrm{psia})$ & 1.4 & 1.4 & 2.0 & 2.0 \\
Predicted $N_{p e}$ & 4.4 & 4.7 & 5.6 & 4.3 \\
\hline
\end{tabular}

and multiple scattering) without compromising safety. The Cherenkov light produced in the tank was reflected off the spherical mirrors. There were two mirrors in the front and three in the rear tanks. All of the mirrors were manufactured for E142 at CERN by slumping a $3 \mathrm{~mm}$ thick $836 \mathrm{~mm}$ diameter disk of float glass into a stainless steel mold [95]. The glass was cut, cleaned, then coated with $80 \mathrm{~nm}$ of $\mathrm{Al}$, followed by a protective coating of $30 \mathrm{~nm} \mathrm{MgF}$. Original CERN measurements of the mirrors reflectivities averaged from $80 \%$ at $160 \mathrm{~nm}$ to $89 \%$ at $200 \mathrm{~nm}$. Image sizes at the focal point were estimated to be $\approx 3 \mathrm{~mm}$ in diameter. After the last usage in the E143 experiment the mirrors were stored in dust tight boxes and kept in a nitrogen atmosphere. The reflectivity of the mirrors is not expected to deteriorate within a few years [96]. It was re-measured in a limited range of wavelengths, as shown in fig. 2.15. The mirrors were rigidly held by aluminum clamps and mounted in the E142 aluminum frame $19 \mathrm{~cm}$ from the downstream end window. In each 


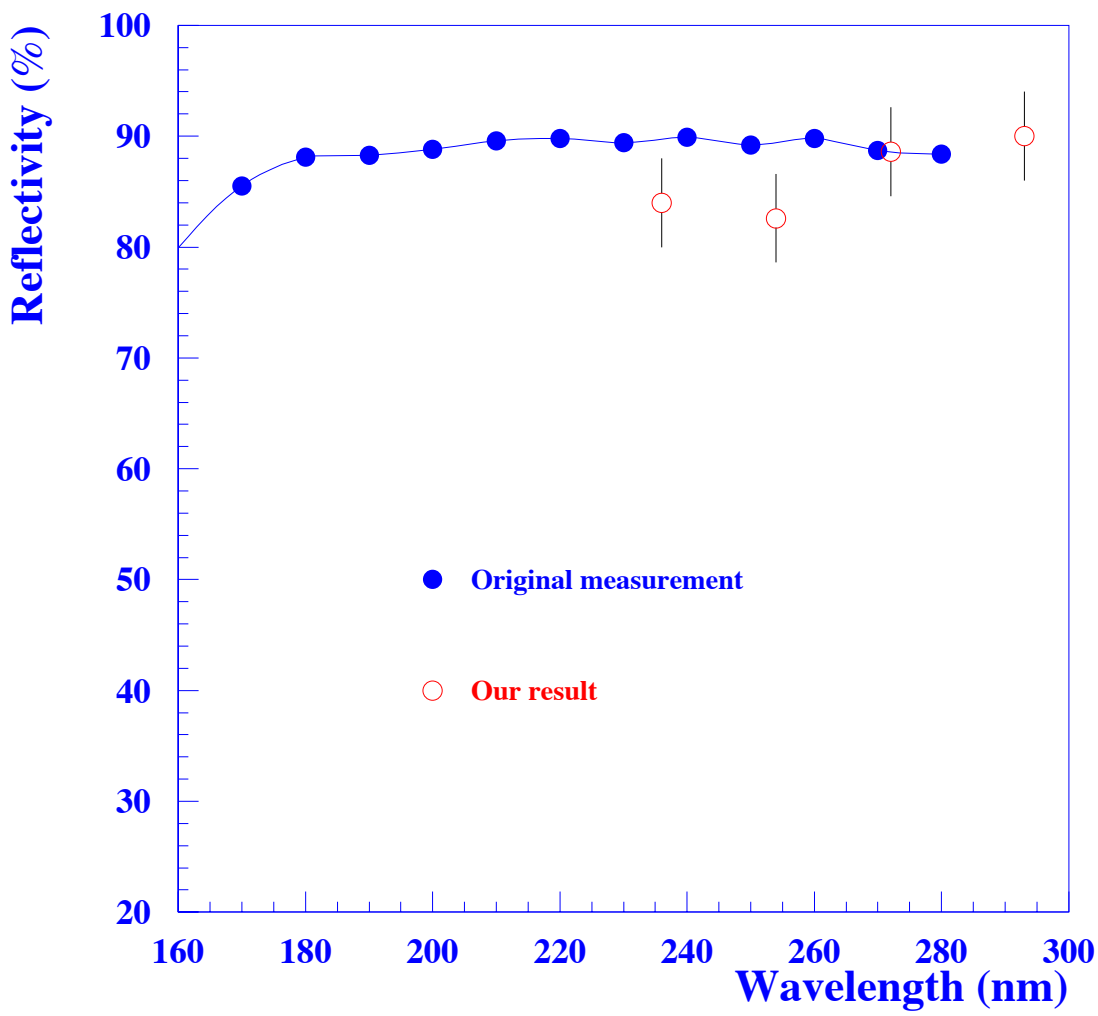

Figure 2.15: Original and remeasured reflectivity of the mirrors.

tank the two or three mirrors were first positioned with respect to each other to constitute one continuous spherical surface with a center perpendicular to the central trajectory. Then they were aligned to focus all of the Cherenkov light onto a single $5 "$ surface of the photomultiplier tube.

We used Hamamatsu 5" UV glass photomultiplier tubes (PMTs), three R1584-01 and one R1584-03. They operated with positive high voltage (HV) bases. Some characteristics of the PMTs are given in table 2.6. The phototubes were not designed to work in a sub-atmospheric pressures. We enclosed 
Table 2.6: Characteristics of Hamamatsu R1584 PMTS.

\begin{tabular}{lc}
\hline Characteristic & Hamamatsu R1584 \\
\hline Spectral Response & $200 \mathrm{~nm}-640 \mathrm{~nm}$ \\
Peak Qquantum Efficiency & $21 \%$ at $380 \mathrm{~nm}$ \\
Photocathode & Bialkali \\
Dynode Structure & 14 stages, linear focus \\
Operating Voltage & $+2500 \mathrm{~V}$ \\
Anode Pulse Rise & $2.2 \mathrm{~ns}$ \\
Transit Time Spread & $1.2 \mathrm{~ns}$ \\
Gain & $\sim 3 \times 10^{7}$ \\
\hline
\end{tabular}

the bases and all the HV connections in vacuum tight cans, built at Temple University. The cans were filled with nitrogen and kept at atmospheric pressure. To reduce the losses caused by the fringe fields of the dipole magnets, primarly a concern for $5 \mathrm{C} 1$, the first Cherenkov counter in $5.5^{\circ}$, in the field of B4 (see fig. 2.13, page 80), $\mu$-metal shelding was used around the PMTs.

To capture the Cherenkov photons in the UV, we coated the faces of the tubes with $2430 \mathrm{~nm}\left(0.3 \mathrm{mg} / \mathrm{cm}^{2}\right)$ of p-terphenyl wavelength shifter, followed by a protective coating of $25 \mathrm{~nm}$ of $\mathrm{MgF}_{2}$ [97]. The film thicknesses were controlled during the vacuum deposition with a crystal thickness monitor. The maximum fluorescence of p-terphenyl is around $370 \mathrm{~nm}$ [98] which well matched the region of high quantum PMT efficiency.

The tanks were filled with the ultra high (99.999\%) purity nitrogen. It was delievered through a Matheson 462 oil and water filter and a Nupro mesh filter in $12.7 \mathrm{~mm}$ diameter electropolishded stainless steel tubing. The evacuating system used 50 cubic feet per minute pumps equipped with a 
molecular sieve and a cold (liquid nitrogen) trap. Such measures were necessary to minimize the possibility of oxygen, water or oil contaminations that would absorb the Cherenkov photons. Both filling and evacuating were controlled remotely from the Counting House. The pressure in the $2 \mathrm{C}$ tanks $\left(2.75^{\circ}\right)$ was 1.4 psia and in the $5 \mathrm{C}$ tanks $\left(5.5^{\circ}\right)$ was 2.0 psia at $22^{\circ} \mathrm{C}$. It was monitored with Setra Model 270 and TransMetric Model P21A transducers accurate to $0.1 \%$. The temperature was monitored with Yellow Springs Instruments Model 4320 Thermilinear thermometers, good to $0.1^{\circ} \mathrm{C}$.

\subsubsection{Predicted response}

The estimation of the number of photo-electrons $N_{p e}$ produced by electrons passing an effective length $L_{\text {eff }}$ of the counter must include several ef-

fects. The emitted Cherenkov photon spectrum is given by eq. (2.20). While travelling in nitrogen of density $\rho$ over an average path length $L_{\gamma}$ the photons are absorbed with the $\sigma(\lambda)$ photo-absorption cross section. Let's denote by $\epsilon$ the collection efficiency of the detector. A loss due to the mirrors' reflectivity is denoted by $R(\lambda)$. We also need to take into account the wavelength shifter conversion $C\left(\lambda, \lambda^{\prime}\right)$ and the quantum efficiency $Q E(\lambda)$ of the photomultiplier cathode. Convoluting all the factors one obtains

$$
\begin{aligned}
N_{p e} & =\epsilon 2 \pi \alpha L_{\mathrm{eff}} \cdot \\
& \cdot \iint_{\beta n>1} d \lambda d \lambda^{\prime}\left[1-\frac{1}{\beta^{2} n^{2}}\right] \frac{R(\lambda)}{\lambda^{2}} \exp \left[-\sigma(\lambda) \frac{\rho L_{\gamma} N_{A V}}{A}\right] C\left(\lambda, \lambda^{\prime}\right) Q E\left(\lambda^{\prime}\right),
\end{aligned}
$$

where $N_{A V}$ is Avogadro's number and A is the molecular mass of $\mathrm{N}_{2}$. 
The quantum efficiency $Q E(\lambda)$ of our phototubes from $\lambda=200 \mathrm{~nm}$ to above $600 \mathrm{~nm}$ has a shallow maximum of $\approx 21 \%$ around $380 \mathrm{~nm}$ [99]. The p-terphenyl wavelength shifter absorbs photons from $115 \mathrm{~nm}$ to $310 \mathrm{~nm}$ and re-emits them at $\lambda_{r}=370 \mathrm{~nm}$ with the spread of $\sigma_{r}=30 \mathrm{~nm}$ [98]. The quantum efficiency $\epsilon_{r}$ of this process is 85\% [100] and for the thicknesses greater than $1000 \mathrm{~nm}$ is roughly wavelength independent [101]. Above $350 \mathrm{~nm}$ the incident photons pass through the wavelength shifter unaltered with a small absorption loss of $\approx 5 \%$ [98]. The re-emmision is isotropic so only a fraction $\epsilon_{g}$ of the photons has a chance to hit the photocathode. Due to the total internal reflection at the nitrogen/p-terphenyl and PMT glass/photocathode boundaries only $\approx 15 \%$ of re-emitted photons will be lost directly, giving $\epsilon_{g}$ somewhere between 0.55 and 0.75 [102]. The conversion function $C\left(\lambda, \lambda^{\prime}\right)$ can be approximated by

$$
C\left(\lambda, \lambda^{\prime}\right)= \begin{cases}\frac{\epsilon_{r} \epsilon_{g}}{\sqrt{2 \pi} \sigma_{r}} \exp \left[-\frac{1}{2}\left(\frac{\lambda^{\prime}-\lambda_{r}}{\sigma_{r}}\right)^{2}\right] & \text { for } \lambda \in[110 \mathrm{~nm}, 310 \mathrm{~nm}] \\ 0.95 \delta_{\lambda, \lambda^{\prime}} & \text { for } \lambda \in[310 \mathrm{~nm}, 640 \mathrm{~nm}]\end{cases}
$$

The collection efficiency $\epsilon$ of the detector reflects inefficiencies such as absorbsion in the PMT glass, losses during photoelectron transit from the photocathode to the dynodes (not included in the $Q E$ of the tube), variations of the efficiency acrosss the face of the PMT. It was inferred from the comparison of predicted and measured $N_{p e}$ in E142 experiment.

Using eq. (2.26) with the input described, we were able to predict the expected number of photoelectrons quoted in table 2.5. The response to pions is a simple modification of the response to electrons, as given in eq. (2.24). 


\subsubsection{Cherenkov electronics}

The signals from the photomultiplier anodes were digitized by the Struck DL515 VME-based Flash ADCs and read by the Data Acquisition System. Four channels of the $250 \mathrm{MHz}$ Flash ADC were interleaved to produce an effective resolution of $1 \mathrm{~ns}$. The last dynode signals were actively fanned out by LeCroy $428 \mathrm{~F}$ Fan-in/fan-out and went to the coincidence scalers in the Counting House and another Fan-in/fan-out that split the signal to four LeCroy 623B discriminators followed by LeCroy 2277 multi-hit TDCs. The TDC signals were used for FADC synchronization. The pressures and temperatures in the tanks were read out by Slow Analog Monitors (SAMs) every five minutes and written on tape.

\subsection{Scintillator hodoscopes}

Each of the spectrometers contained two sets of scintillator hodoscopes located at the rear of the Cherenkov counters (see fig. 2.13). They were used

for the purpose of tracking the momenta of the established particles. There were six front hodoscope planes, denoted as 2H1-2H6, and four back planes, 2H7-2H10, in $2.75^{\circ}$ spectrometer, and four front, 5H1-5H4, four back, 5H5$5 \mathrm{H} 8$, hodoscope planes in the $5.5^{\circ}$ spectrometer. All the $5 \mathrm{H}$ planes as well as 2H5Y, 2H6X, 2H9Y, 2H10X were adepted from E142/E143 hodoscopes [103] without major modifications. The fingers of these hodoscopes overlapped by $1 / 3$ of their width to improve spatial resolution. Six new planes were built for the $2.75^{\circ}$ arm. Two new planes, $2 \mathrm{H} 1 \mathrm{U}$ and $2 \mathrm{H} 2 \mathrm{~V}$, were tilted by $15^{\circ}$ relative 
to the horizontal direction. Their fingers were two times narrower than the old ones, overlapped by $1 / 3$. They were wrapped in black electrical tape and enclosed in a light-tight box. Another four new planes, $2 \mathrm{H} 3 \mathrm{X}$ and $2 \mathrm{H} 4 \mathrm{Y}$ in the front and $2 \mathrm{H} 7 \mathrm{X}$ and $2 \mathrm{H} 8 \mathrm{Y}$ at the back, with narrow fingers, were split and light isolated in the middle and had a separate phototube for each half. They overlapped by $1 \mathrm{~mm}$. All planes were positioned perpendicularly to the central ray. The geometry of the additional hodoscopes is given in table 2.7.

Table 2.7: Geometrical Parameters of E154 Hodoscopes.

\begin{tabular}{lrcccc}
\hline Plane & $\begin{array}{r}\text { Horiz. } \\
\text { angle } \\
\left({ }^{\circ}\right)\end{array}$ & $\begin{array}{c}\text { Area } \\
x \times y\end{array}$ & $\begin{array}{c}\text { No. of } \\
\text { active } \\
\text { channels }\end{array}$ & $\begin{array}{c}\text { Finger } \\
\text { width } \\
(\mathrm{mm})\end{array}$ & $\begin{array}{c}\text { Finger } \\
\text { thickness } \\
(\mathrm{mm})\end{array}$ \\
\hline $2 \mathrm{H} 1 \mathrm{U}$ & +15 & $360 \times 370$ & 44 & 15 & 6 \\
$2 \mathrm{H} 2 \mathrm{~V}$ & -15 & $360 \times 370$ & 44 & 15 & 6 \\
$2 \mathrm{H} 3 \mathrm{X}$ & 90 & $363 \times 412$ & 56 & 13 & 13 \\
$2 \mathrm{H} 4 \mathrm{Y}$ & 0 & $362 \times 413$ & 64 & 13 & 13 \\
$2 \mathrm{H} 5 \mathrm{Y}$ & 0 & $430 \times 589$ & 25 & 30 & 6 \\
$2 \mathrm{H} 6 \mathrm{X}$ & 90 & $430 \times 589$ & 31 & 20 & 6 \\
$2 \mathrm{H} 7 \mathrm{X}$ & 90 & $513 \times 992$ & 82 & 13 & 13 \\
$2 \mathrm{H} 8 \mathrm{Y}$ & 0 & $512 \times 993$ & 72 & 25 & 13 \\
$2 \mathrm{H} 9 \mathrm{Y}$ & 0 & $510 \times 1070$ & 54 & 30 & 6 \\
$2 \mathrm{H} 10 \mathrm{X}$ & 90 & $510 \times 1070$ & 27 & 30 & 6 \\
$5 \mathrm{H} 1 \mathrm{U}$ & -45 & $430 \times 690$ & 25 & 45 & 6 \\
$5 \mathrm{H} 2 \mathrm{X}$ & 90 & $430 \times 690$ & 23 & 30 & 6 \\
$5 \mathrm{H} 3 \mathrm{Y}$ & 0 & $430 \times 690$ & 36 & 30 & 6 \\
$5 \mathrm{H} 4 \mathrm{~V}$ & +45 & $430 \times 690$ & 25 & 45 & 6 \\
$5 \mathrm{H} 5 \mathrm{U}$ & -45 & $527 \times 1064$ & 21 & 75 & 10 \\
$5 \mathrm{H} 6 \mathrm{X}$ & 90 & $510 \times 1070$ & 27 & 30 & 6 \\
$5 \mathrm{H} 7 \mathrm{Y}$ & 0 & $510 \times 1070$ & 55 & 30 & 6 \\
$5 \mathrm{H} 5 \mathrm{U}$ & +45 & $527 \times 1064$ & 21 & 75 & 10 \\
\hline
\end{tabular}


The fingers were made of Bicron BC404 scintillator. It has a 1.58 index of refraction, a very fast rise time of $0.7 \mathrm{~ns}$ and a short pulse of $2.2 \mathrm{~ns}$ FWHM. They were individually wrapped in aluminum foil and black electrical tape. Most of them were connected to the 1/2 inch Hamamatsu R4014 phototubes (PMT's) characterized by a short, $1.1 \mathrm{~ns}$ risetime and a small, $0.5 \mathrm{~ns}$ transit time spread. The $2.75^{\circ}\left(5.5^{\circ}\right)$ PMT anode signals were processed by LeCroy 3412( 4413) discriminators, then by LeCroy 3377 (2277) multi-hit TDC's and

finally read out by the DAQ system. There were a total of 499 (233) active channels.

\subsection{Electromagnetic shower counter}

The energy of the electrons was measured by the electromagnetic calorimeters [104] (shower counters) in a fly's eye configuration, located at the ends of the spectrometers. Each calorimeter consisted of an array of 20 rows and 10 columns of $\mathrm{F} 2$ lead blocks. The face of each block was $6.2 \mathrm{~cm}$ by $6.2 \mathrm{~cm}$ and the length was $75 \mathrm{~cm}$. The blocks were wrapped in an aluminum foil and two layers of a black tape.

The high energy electrons entering the shower lose their energy by the Bremsstrahlung of energetic photons which in turn creates $e^{+} e^{-}$pairs. This produces a shower of a large number of electrons and photons. The average energy loss by Bremsstrahlung is governed by the radiation length, $X_{\circ}=$ $3.17 \mathrm{~cm}$ for $\mathrm{F} 2$ lead glass. After a distance $X_{\circ}$ the electron energy is $1 / \mathrm{e}$ of the original. The characteristic pair production length by a high energy 
photon is $(7 / 9) X_{0}$. The longitudinal energy shape is thus dictated by the radiation length $X_{\circ}$ and is given by [105]

$$
\frac{d E}{d z} \sim\left(\frac{z}{X_{\circ}}\right)^{\alpha} e^{-z /\left(\lambda X_{0}\right)},
$$

where $\lambda=1.9$ and $\alpha=\log (E / 13.9 \mathrm{MeV}) / \lambda(13.9 \mathrm{MeV}$ is the critical energy below which the ionization dominates the Bremsstrahlung). The energy loss peaks around $7 X_{\circ}$, and all the shower is contained in the length $24 X_{\circ}$ of our calorimeter. The transverse energy shape is highly regular, determined by the Moliére radius, $R_{m} \approx 5 \mathrm{~cm}$ :

$$
\frac{d E}{d r} \sim e^{-4 r / R_{m}}
$$

and is usually contained in 9 blocks. The electrons and positrons in the shower produce Cherenkov light in the lead glass, which has index of refraction 1.62. The light was collected by 2 inch Amperex XP2212PC photomultipliers attached to the rear of the blocks. The anode signal was directed by a passive splitter to the LeCroy 2282 12-bin ADC and usually to a LeCroy 4413 discriminator and then to a 2277 multihit TDC. The 64 blocks with the highest instantaneous rates used three LeCroy 3412 discriminators with increasing thresholds and three 3377 TDCs. The TDCs operated in the 'burstguard' mode, and both edges of the discriminator output were detected. This allowed the use of timing information in the energy reconstruction of the overlapped events.

The hadronic showers are characterized by an interaction length, $\lambda_{i}$, which equals $34 \mathrm{~cm}$ for our lead glass. Thus the calorimeter is less than 2 interaction 
lengths long and only $3 \%$ of the pions deposit 80 to $100 \%$ of their energy [106]. About $17 \%$ of them do not shower, leaving only a minimum ionizing energy $0.5 \mathrm{GeV}$. The broad deposition peak is centered around $38 \%$ of the pion energy. The transverse profile of the pion shower is broad (in comparison to the electron). It is also irregular, due to a small number of produced particles. Some of the energy can be converted to $\pi^{\circ}$ 's via charge exchange processes like $\pi^{-} p \rightarrow \pi^{\circ} n$. The decay of $\pi^{\circ}$ into two photons produces an electromagnetic shower. If the conversion to $\pi^{\circ}$ is later in the shower process, then the resulting transverse profile is asymmetric. All the differences between the transverse shapes of the electron and the pion showers may be used for their discrimination.

\subsection{Data acquisition (DAQ)}

The E154 experiment did not use the trigger per se due to the short SLED beam pulse. The electronics was started and stopped (TDCs, FADCs) or gated (ADCs) on the $\mathrm{A} 2 \mathrm{~N}$ accelerator timing signal. The $\mathrm{A} 2 \mathrm{~N}$ signal was generated at the source at $120 \mathrm{~Hz}$.

Prior to E154 the data acquisition used in the ESA was based on a DEC MicroVAX 4000-200 with a "Qbus" CAMAC. The data transfer was limited (by CAMAC speed and single bus architecture) to $300 \mathrm{kB} / \mathrm{s}$, which was not adequate for the beam triggered experiments. The new DAQ system [107], based on VME crates, was designed to handle transfer rates up to $\sim 1 \mathrm{Mb} / \mathrm{s}$. A block diagram of the system is shown in fig. 2.16. The front end of the 


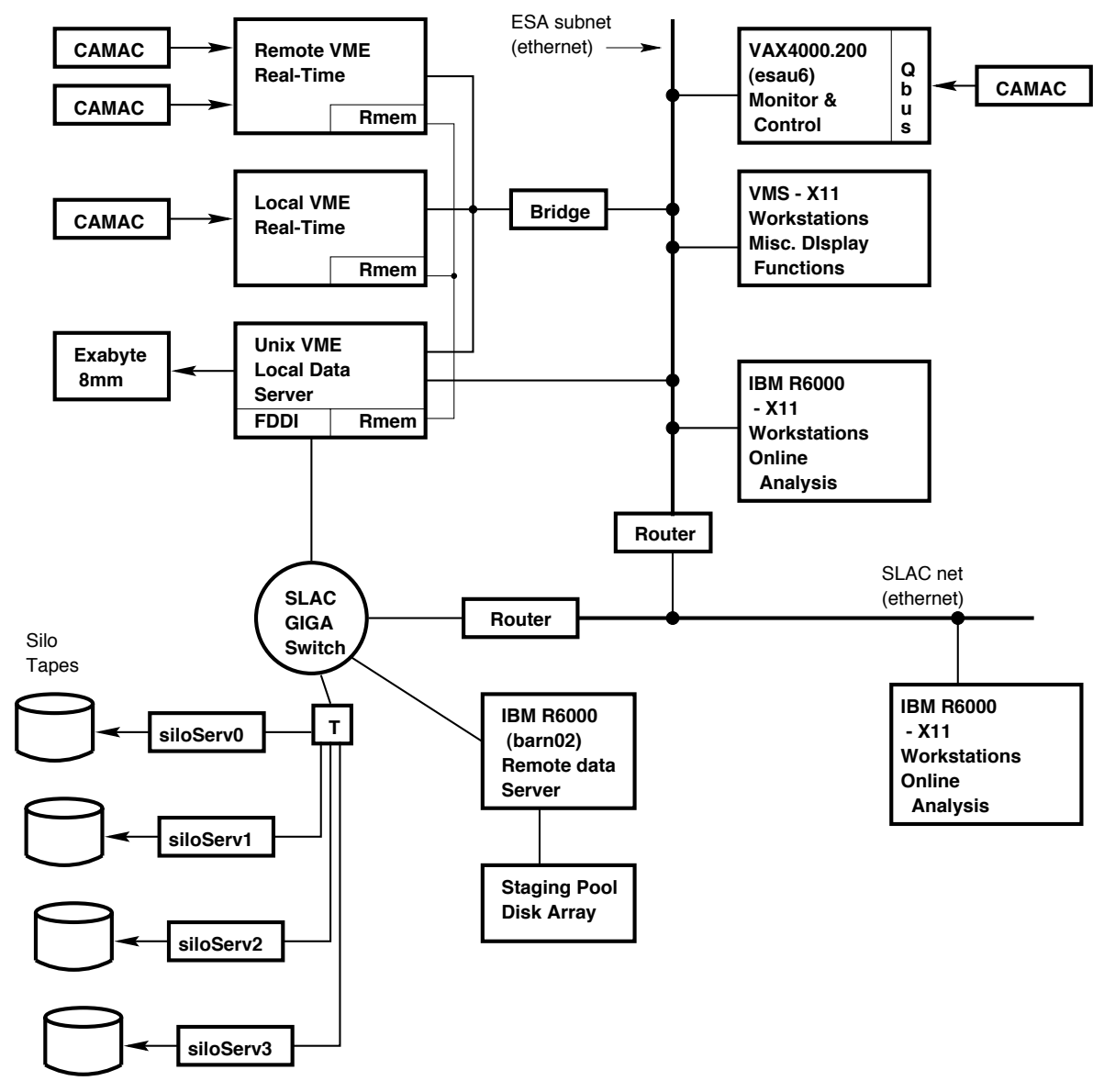

Figure 2.16: The VME-based Data Acquisition system for ESA.

DAQ consisted of three VME crates. Two of them, the local VME crate (located in the Counting House) and the remote one (located in the $2.75^{\circ}$ detector hut) housed the real-time components of the DAQ system. The third Unix VME crate (located in the Counting House) contained Unix processors and interfaces to the data storage system. The crates were linked 
via a reflective memory subsystem that allowed the data to be shared by all three of them. The overall control and monitoring was performed by the VAX 4000-200 computer equipped with Qbus-CAMAC interface located in the Counting House. It issued the start and stop commands to the real-time components using the TCP/IP networking protocol. It also controlled the target position, High-Voltage, run type (pedestal, Møller, normal, etc.), logging and monitored power supplied voltages to NIM and CAMAC electronics and Cherenkov gas pressure.

The remote VME crates contained interfaces to each of the two spectrometers CAMAC branches and VME-based FADCs. The local crate had a CAMAC interface to the beam data branch. Each crate was operated by the MVME166 real-time processor that controlled the raw data readout and writing to the reflective memory network. The Unix VME contained two MVME197 processors. One was used for the real-time application software and provided the monitoring environment for the real-time processors. The other processor ran a Data Server job with the main task of shipping the data via MVME385 FDDI interface to the SCS (SLAC Computer Center) Silo, or when the Silo system was not available, record the data on a local $8 \mathrm{~mm}$ tape subsystem (there was no need during E154 to use the last possibility). It also sent a fraction of the data over the network to any number of analysis jobs running on a variety of workstations, two IBM RS6000 among them, dedicated to the on-line analysis. The maximum data transfer rate in E154 
was about $750 \mathrm{kB} / \mathrm{s}$, limited by the $\mathrm{CAMAC}$ readout that did not allow one to achieve the design goal of $1 \mathrm{MB} / \mathrm{s}$.

The Silo storage system worked very well. The FDDI network provided transfer rates on the level of $2 \mathrm{MB} / \mathrm{s}$. The storing process was operated by an IBM R6000 server at SCS that stored the data on disk temporarily storage, and after the run was completed, issued the command to the silo robot to save the data on 1 GB silo tapes. These tapes were available for offline analysis through the same automated Silo System. After the run the full data set was copied onto 5 GB $8 \mathrm{~mm}$ tapes and used by the Caltech based analysis team. 


\section{CHAPTER 3}

\section{DATA ANALYSIS AND RESULTS}

The E154 experiment was the first to run with the energy of the polarized electron beam of $48.3 \mathrm{GeV}$. Three beam currents were used, $9 \times 10^{10}$, $5 \times 10^{10}$ and $3 \times 10^{10}$ electrons per spill, at the rate of $120 \mathrm{~Hz}$. The typical run consisted of 200,000 spills. There were about 1800 runs stored on magnetic tapes, 1.4 Tbytes of data, that were used for various analyses. They came from nine polarized targets and four reference cells. The electron rates were approximately constant, 0.5 per spill, in the $2.75^{\circ}$ spectrometer, and were changed with the current changes, $0.2-0.07$ per spill, in the $5.5^{\circ}$ spectrometer. That resulted in the total of 100 million deep inelastic events.

The analysis was performed in two stages. The raw data tapes were analyzed first, and the information about the beam, Cherenkov hits, shower clusters and found tracks was written on Data Summary Tapes (DSTs). The DST production at Caltech used four DEC Alpha 600 5/266 workstations 
and lasted seven weeks. Next the DST tapes were processed to identify the electrons, calculate their kinematics and bin them in $x$ and $Q^{2}$ separately for each beam-target spin configuration. The outcome was stored in the summary files that were directly used for asymmetry and structure function computations.

There were two independent analyses of the E154 data, based at SLAC and at Caltech. The results of both groups agreed very well. They were averaged for the final, published, E154 results. In this chapter the details of the Caltech team analysis will be presented.

\subsection{Cherenkov analysis}

The Cherenkov detectors provided a basic tool for electron identification and pion rejection. The coincidence signals from two detectors and from the shower counter were a powerful input for the tracking algorithm. The outputs from the Cherenkov photo-multipliers were digitized by Flash ADCs (FADCs) . Four channels of the $250 \mathrm{MHz}$ FADC were interleaved to produce an effective resolution of 1 ns. An example of the FADC waveform is shown in fig. 3.1. The waveform was processed by the software that singled out the pulses coming from different hits and established their time and amplitude (or equivalently, total charge, i.e. area). The total charge, corresponding to the channel number if typical ADC was used, is directly proportional to the number of photoelectrons emitted from the photo-multiplier cathode. 


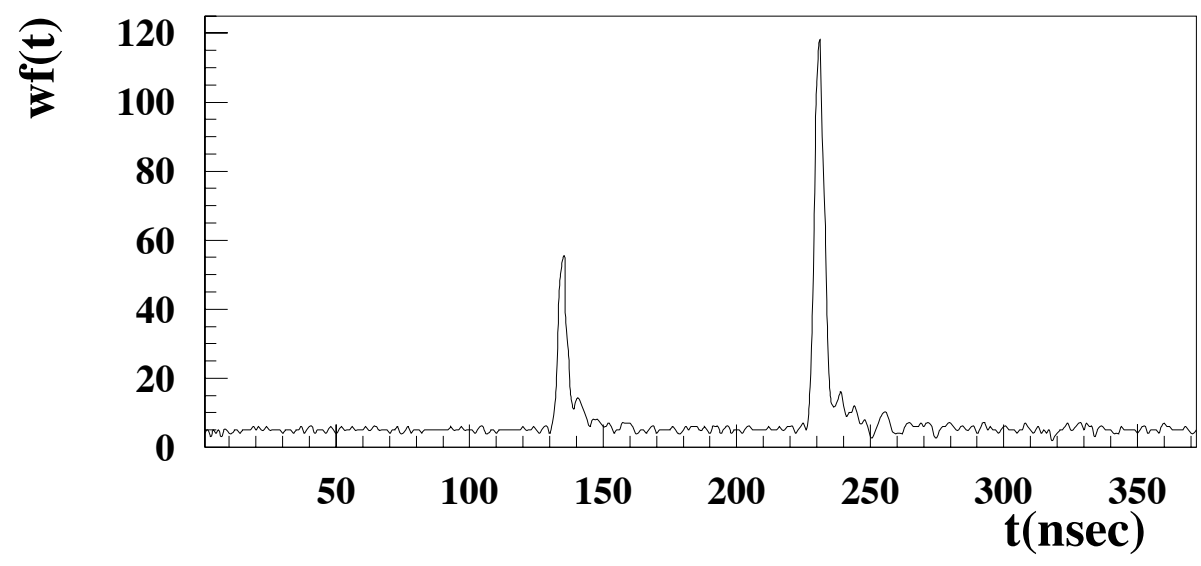

Figure 3.1: An example of a Cherenkov signal digitized by FADC.

The essential ideas of the software algorithm are as follows: The time derivative of the waveform is calculated first. That gives the rough location of the single pulses. Next the average flat baseline is computed in the regions away from the pulses and then subtracted out from the waveform. Now the time of the found pulses is determined more precisely. We start with the first pulse and determine its time, amplitude and the integrated charge. Before proceeding with the next pulse we subtract out the previous pulse from the waveform by scaling in amplitude the stored average waveform. This is because different phototube signals are related to each other via constant amplitude scaling factors, while the time structure remains unchanged. Thus the areas, i.e. integrated charges, are also related via the same factor. That allows one to compute the full charge even if the subsequent pulse interrupts the whole pulse range integration (the piece in the superimposed part is taken from the average waveform), and to correct for the saturated pulses, those 
that have larger amplitude than the FADC range. The timing information is inferred from the position of the half maximum point and then corrected for the pulse hight (for slewing).

The time resolution of the above algorithm was subject to the 4 ns time jitter of the FADC clock, adding $4 / \sqrt{12} \approx 1.2$ ns to the resolution. The 250 MHz clocks of FADCs were not synchronized. The solution [108] was to synchronize the FADCs signals by the TDCs clocked at $1 \mathrm{GHz}$. The resolution was improved from $1.4 \mathrm{~ns}$ to about $0.8 \mathrm{~ns}$, as seen in fig. 3.2. That resulted
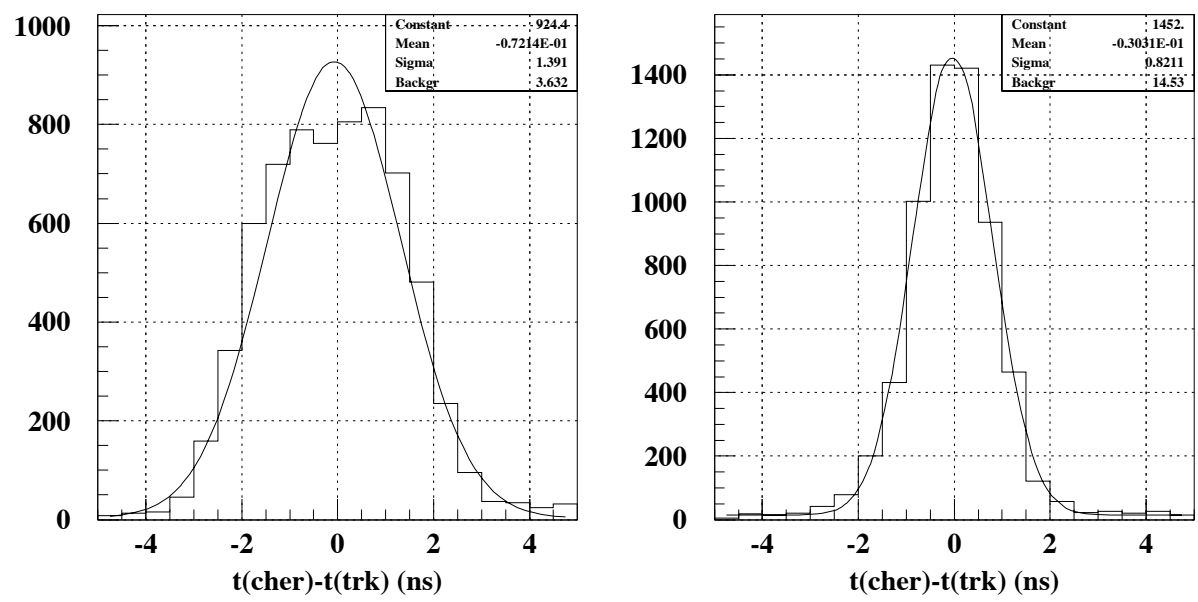

Figure 3.2: The time resolution of unsynchronized (left) and TDC synchronized (right) FADC.

in a reduced random background leading to smaller pion contamination.

A typical response of the Cherenkov detectors to electrons and pions is shown in fig. 3.3. The average number $N_{p e}$ of photoelectrons and the amplitude of one photo-electron signal $V_{1 p e}$ in FACD units for each counter 


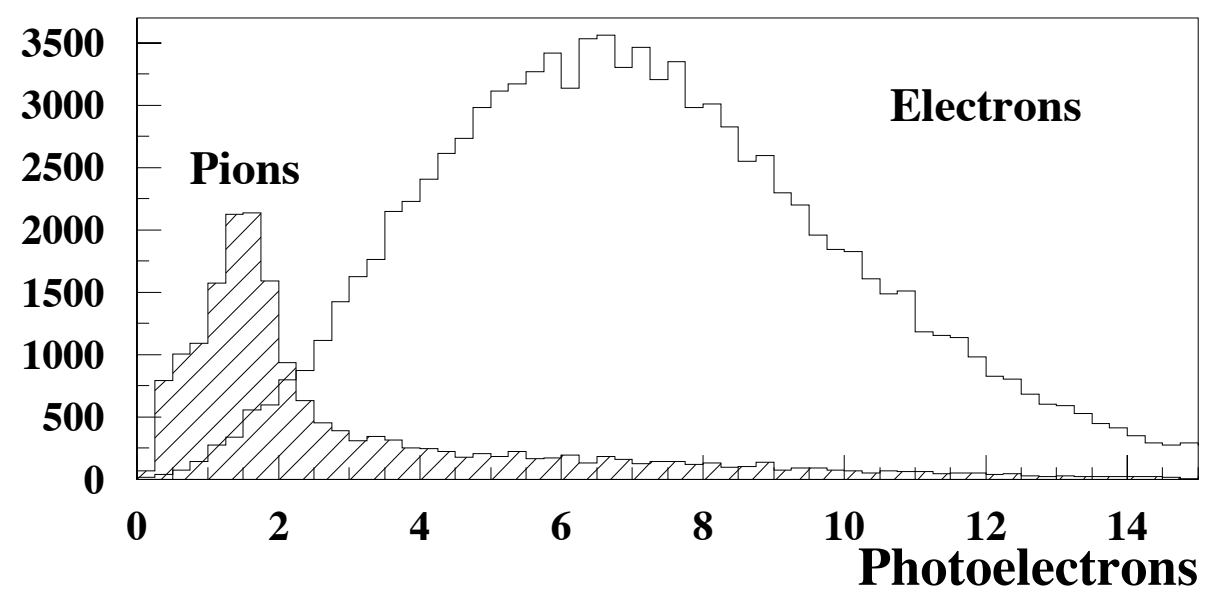

Figure 3.3: Typical Cherenkov response to electrons and pions.

Table 3.1: Cherenkov Response to Electrons.

\begin{tabular}{lcccc}
\hline Tank & $2 \mathrm{C} 1$ & $2 \mathrm{C} 2$ & $5 \mathrm{C} 1$ & $5 \mathrm{C} 2$ \\
\hline$N_{p e}$ & 5.7 & 5.1 & 6.2 & 5.0 \\
$V_{1 p e}$ & 16.8 & 14.1 & 12.1 & 13.6 \\
\hline
\end{tabular}

is given in the table 3.1 [109]. From the previous pulse scaling considerations it is obvious that the pulse hight $V_{\max }$ determines the $N_{p e}$ in a simple manner, $N_{p e}=V_{\max } / V_{1 p e}$. The Cherenkov efficiency was found to be around $95 \%$ [110] and was limited by the intrinsic pulse height cutoff of the algorithm of $4-6$ FADC units and the algorithm dead time of about 5 ns. The efficiency for a typical cut used in the analysis is about $90 \%$ [91]. 


\subsection{Energy determination: Shower analysis}

The shower counter provides us with the measurement of the energy and

electron identification via the $E / p$ ratio and the transverse energy profile of the shower. It also gives the time and the position of the cluster which are the basis for the tracking determination of the momentum and angles. The calorimeter consisted of $10 \times 20$ isolated blocks from which light was collected by the photo-multipliers (cf. sec 2.9, page 94). We record the times of both the leading (LE) and trailing (TE) edges of photo-multiplier pulses. The time of the hit is determined by its LE time. Energies of all hits deposited in one block add up to the total ADC energy of that block in a given spill.

The shower analysis was performed in the following steps [111]:

- Search the calorimeter for the central blocks which are the local energy maxima with

- at least one TDC hit, and

- satisfy in 9 blocks the minimum energy requirement,

$$
\sum_{3 \times 3} E_{i}>\left(E_{\text {cut }}=C_{\text {cut }} p_{\min }(\mathrm{raw})\right)
$$

where $C_{\text {cut }}$ is some constant $\left(0.7\right.$ for electrons) and $p_{\min }$ (raw) is the lowest momentum of the accepted electrons in a given row (constant $9 \mathrm{GeV}$ was used).

- Add to the cluster initiated by the central block any of the surrounding 8 blocks that either 
- are in time with the central block, or

- have no TDC hits.

- From 16 blocks outside $3 \times 3$ array add to the cluster those that either

- are in time with the central block, or

- have no TDC hits and no other cluster nearby.

- Share the energy in the pileup cases (i.e. multiple TDC hits in one block):

- from the leading and trailing timing TDC edges compute the expected energy

$$
e_{i}=f\left(t_{i}^{\mathrm{TE}}-t_{i}^{\mathrm{LE}}\right)
$$

and the corresponding error $\sigma\left(e_{i}\right)$ for every hit $i$

- minimize

$$
\chi^{2}=\sum_{i}\left(\frac{E_{i}-e_{i}}{\sigma\left(e_{i}\right)}\right)^{2}
$$

with a constraint $\sum_{i} E_{i}=E_{\text {total }}$ to find the cell energies $E_{i}$ associated with each $i$ hit.

- Calculate the cluster position from the ratio $E_{\text {side }} / E_{c . b l o c k}$ of the side block to central block energies using the fitted function $f_{\text {pos }}$,

$$
x=f_{\text {poss }}\left(E_{\text {side }} / E_{\text {c.block }}\right)
$$


- Compute the cluster time by averaging the TDC information from all blocks with more than $10 \%$ energy of the central block

$$
t=\frac{\sum_{i} t_{i} / \sigma^{2}\left(t_{i}\right)}{\sum_{i} 1 / \sigma^{2}\left(t_{i}\right)},
$$

where $\sigma\left(t_{i}\right)$ is the time uncertainty for each block.

- Iterate the shower position and energy to get the best shower shape fit.

- Use the shower shape to correct for leakages of the edge blocks.

- After the track has been associated with the cluster, use its position at the shower and fit the shower shape once more to get the final cluster energy and the fit $\chi^{2}$.

- Use the above $\chi^{2}$ as a particle identification.

The main emphasis in the code was put on the energy sharing of overlaps of electron and pion clusters. Such overlaps create rate-dependent biases in energy and position reconstruction and electron identification which have the potential to alter the experimental asymmetries. The simple energy determination from the TE and LE time information has a limited dynamic range, as seen in fig. 3.4. Useful information can be extracted if the ratio of the pulse hight $E$ to the discriminator threshold $E_{t h}$ is smaller than 4 . The electron pulses are often much higher, and thus it was important first to adjust the energies to add up to the total deposited energy and then to refer to the energy shape (cf. fig. 3.5) and multiple iterations to have the best energy determination in the high rate environment to minimize the rate 

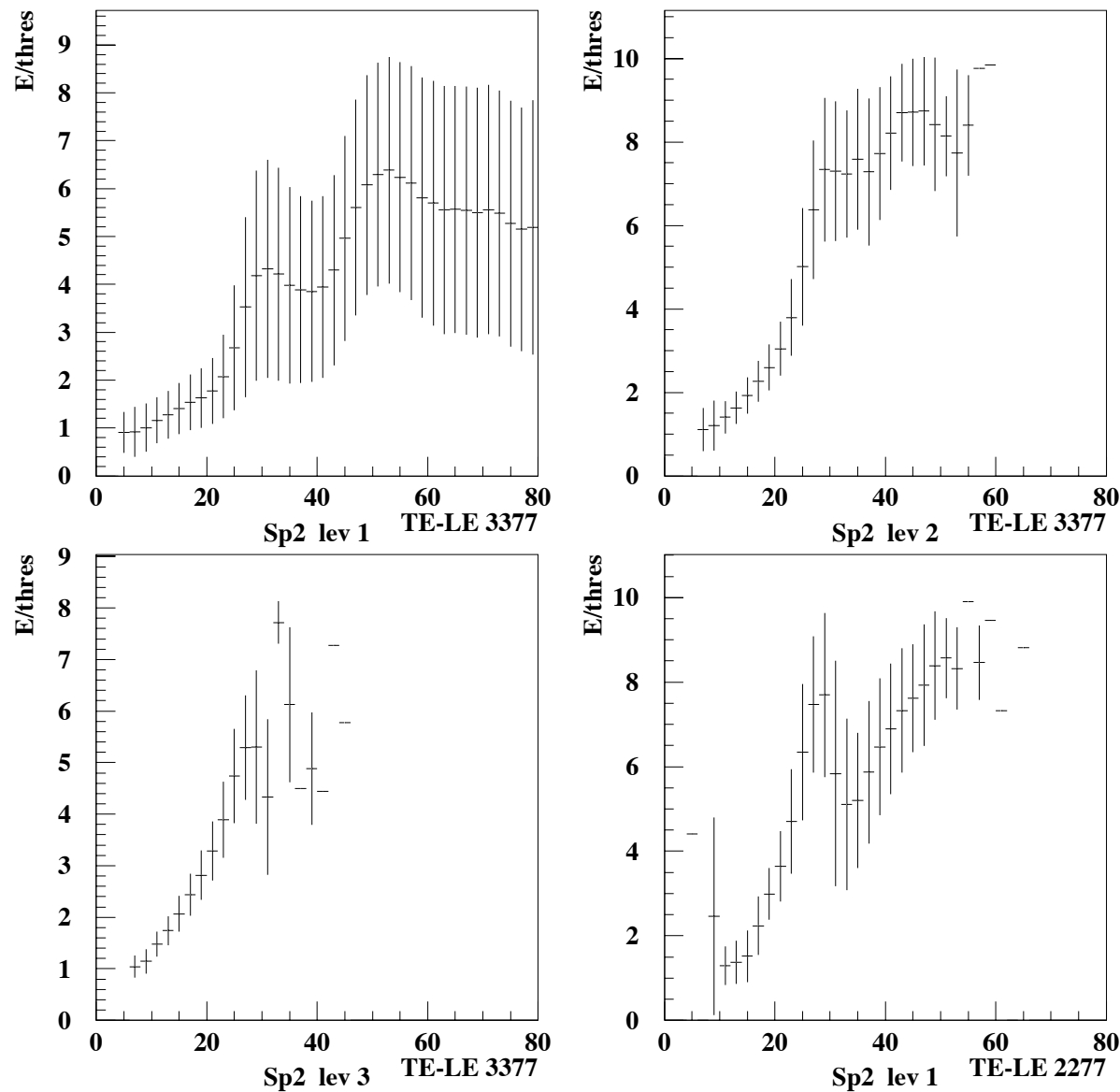

Figure 3.4: The pulse height as a function of the difference between the leading and trailing edge times for different discriminator levels. The error bars represent the RMS of the distribution.

dependence introduced by the $E / p$ cut. Also the use of the fit to the position, $f_{\text {pos }}$, as a function of the side to central block energy ratios (rather than the linear energy weighted position average, cf. fig 3.6) and then iterations for the best shower shape fit helped to establish a better and less biased and thus less rate dependent position resolution (which was $6-8 \mathrm{~mm}$ ), which is 

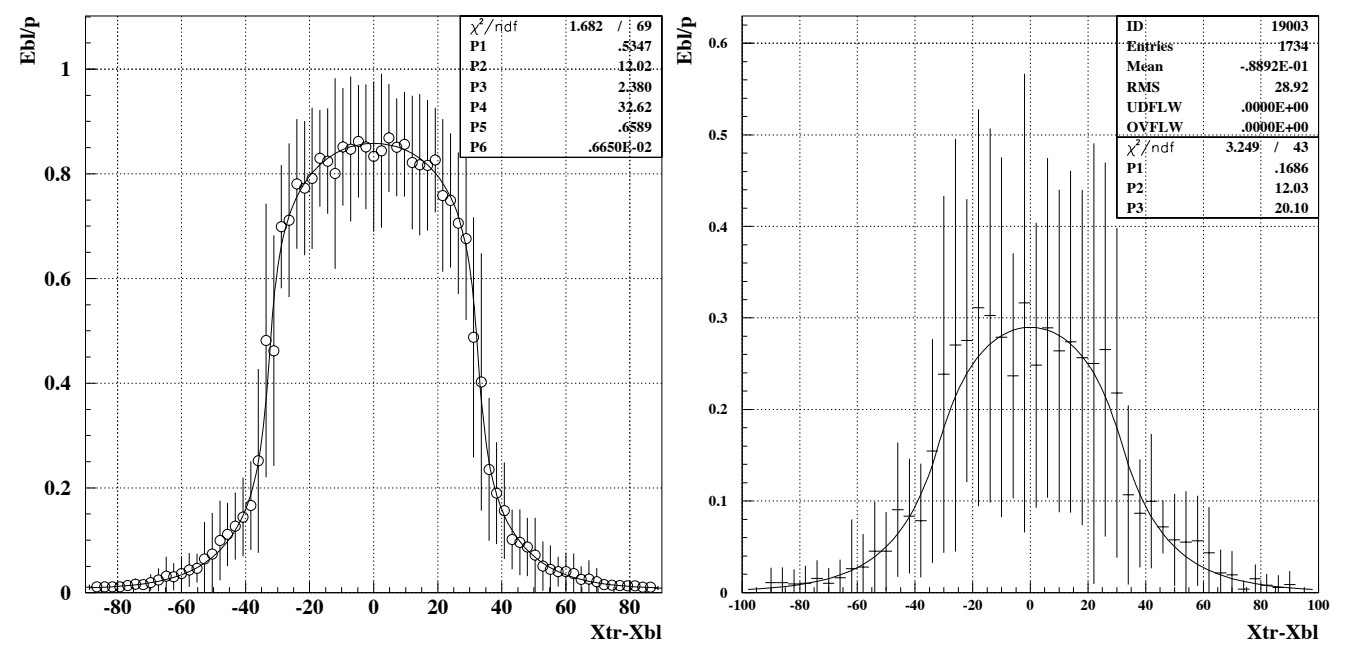

Figure 3.5: Electron (left) and pion (right) shower energy shapes. The error bars represent the spread of the distributions.

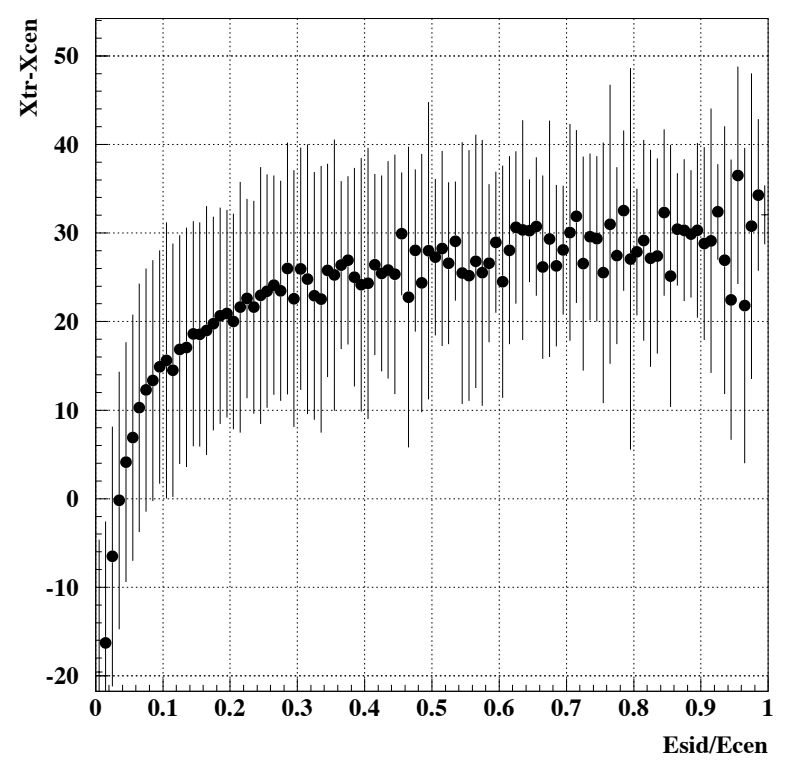

Figure 3.6: The cluster position as a function of the side to central block energy ratio. The rror bars represent the RMS of the distribution. 
directly related to the angular and momentum resolution from tracking. The rejection of the small clusters, with less than $10 \%$ of the central block energy, during the time of cluster determination resulted in a smaller time jitter and together with iterations lead to $0.7 \mathrm{~ns}$ resolution. Finally the shower shape was used to identify electrons $(\chi \leq 0.045)$ via $\chi^{2}$ (cf. fig 3.7$)$ of the fit. The

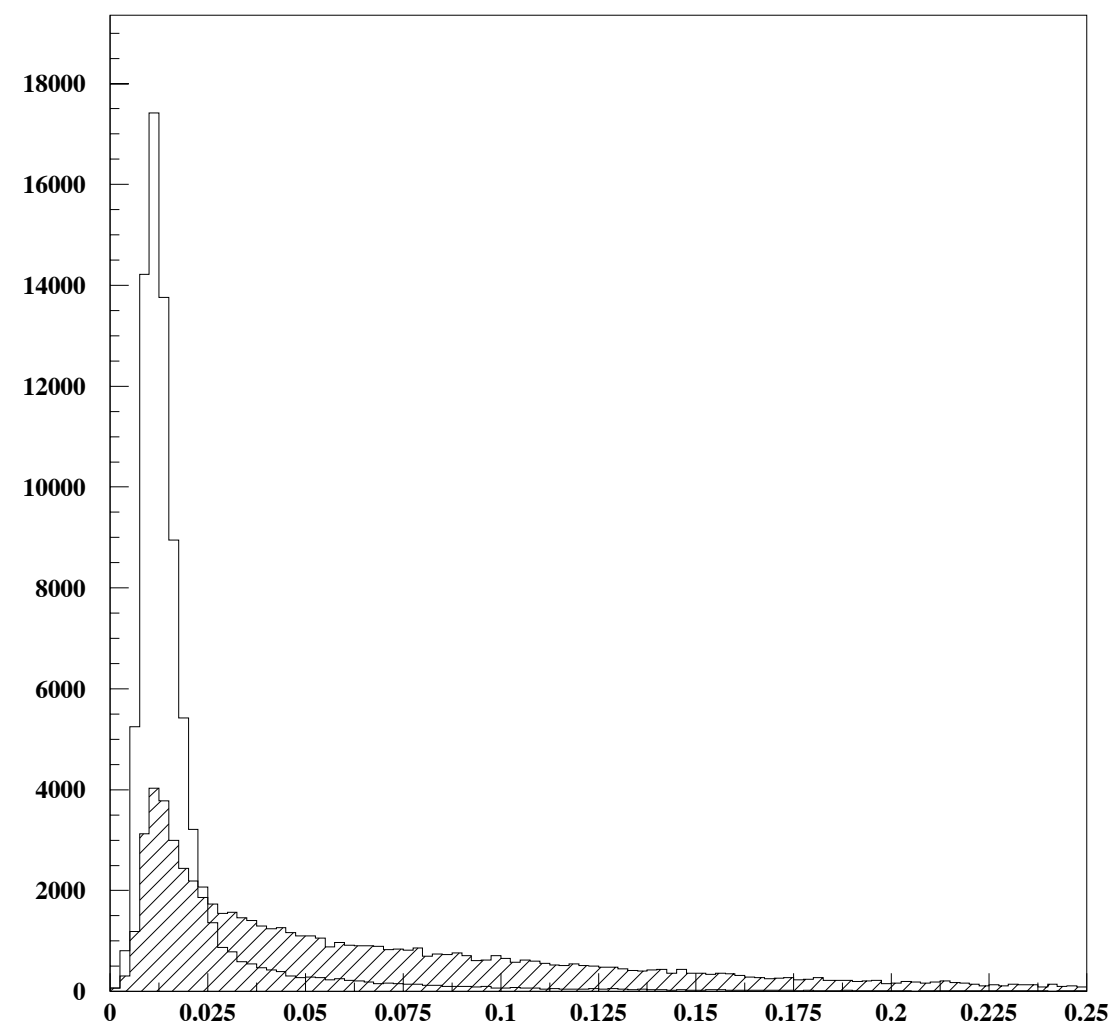

Figure 3.7: Distribution of the shower shape variable $\chi$ for electrons (open area) and pions (hatched area).

pion rejection of this cut was $2: 1$ in the $E / p>0.8$ region, and its electron efficiency ranged from $92 \%$ to $95 \%$ (run dependent) at low $x$ and slowly increased with momentum. 


\subsection{Momentum determination: Tracking}

The information from the Cherenkov detectors, hodoscopes and from the calorimeter was used to find tracks of charged particles and to determine their

momentum $\vec{p}$. The basic definition of an electron required the existence of the track and relied on its parameters. The momentum was used to calculate the kinematics ( $x$ and $Q^{2}$ ) of an event and in making the $E / p$ cut needed for electron identification.

The tracks were classified into four exclusive classes that had associated with them:

1. A shower cluster, at least one Cherenkov signal and at least a certain minimum number of hodoscope hits;

2. A shower cluster and hodoscope signals; no Cherenkov hits were found within the time coincidence window;

3. At least one Cherenkov hit and hodoscope signals; no shower cluster found within the time and space coincidence windows;

4. Hodoscope hits only.

The class 1 tracks are electron candidates, and class 2 tracks are mostly pions. Tracks from class 3 were primarily used for the calorimeter calibration where the cluster information is intentionally removed from tracking to eliminate biases. Class 4 was used for diagnostics. 
The algorithm contained the following steps [91]:

- Copy information from detectors to local common blocks;

- Transform all timing information $t$ to times $\tau$ at the "target", $\tau=$ $t-z / c$, where $z$ is the hit position;

- Collect hit characteristics: shower time and position, and their uncertainties, Cherenkov times and uncertainties, positions and uncertainties of the hit hodoscope fingers. The hodoscopes are arranged into packages corresponding to front and rear hodoscopes in $2.75^{\circ}$ and $5.5^{\circ}$.

- Begin with the shower and loop over shower clusters that are identified as electrons by shower particle ID algorithm, and then over others;

- Calculate the optics cuts. The irection of the momentum is strongly correlated with the shower position for tracks originating at the target. Compute the ranges of slopes $\Delta \Theta$ and $\Delta \Phi$ for the track to originate at the target and translate them onto the allowable hodoscope region;

- Search for the Cherenkov hits within three $\sigma$ of the combined shower and Cherenkov uncertainties. Pick at most 2 hits that minimize the time $\chi^{2}$. Calculate the average time and its uncertainty $\sigma_{t}$;

- Select the hodoscope hits that pass the optics cuts and are sufficiently close to the average time; 
- Project fingers onto the last plane within each package along the line from the center of a finger to the shower cluster position;

- Arrange the hits within a hodoscope package into local clusters;

- Fit the tracks for the time $\tau_{0}$, the position $x_{0}, y_{0}$ and the slopes $\Theta=$ $d x / d z, \Phi=d y / d z$ at the $z=0$ position. First minimize the time $\chi^{2}$, then the total space and time $\chi^{2}$;

- Correct the detector time for the signal position from the knowledge of the track parameters;

- Select the best track candidate: calculate spatial and time $\chi^{2}$ residual for every Cherenkov and hodoscope hit. If the residual is greater than the maximum, the hit is dropped and the fit is repeated. If the remaining hits do not satisfy the minimum track criteria, the whole track is dropped;

- Choose the best $\chi^{2}$ per degree of freedom track from all candidates associated with the same shower cluster;

- Mark the hits from the fitted track to prevent them from being used in another track;

- Create "fake" shower clusters for class 3 and 4 tracks; The spill is divided into 6 ns intervals, and the "fake" clusters are created with infinite position resolution. The priority is given to those clusters surrounded by most of the Cherenkov or hodoscope hits. Then the algorithm is 
used as for real clusters except that the "fake" cluster is not used in fitting;

- Calculate the amplitude of the momentum, horizontal and vertical angles, position and time at the target from the track parameters using tabulated, so called, inverse matrix elements.

The efficiency of the tracking was established from the Monte Carlo technique. Electron tracks were generated using the model of the optics of the spectrometer and then were inserted among the real data with resolution and dead time effects. They were next reconstructed by the standard analysis code. The efficiency, determined as the ratio of the number of reconstructed tracks with $0.8 \leq E / p \leq 1.25$ to the number of seeded tracks, is shown in fig. 3.8. The results were consistent with the inefficiency defined as the ratio $\left(N_{\text {clusters }}-N_{\text {tracks }}\right) / N_{\text {clusters }}$. The main contributions to the inefficiency for $2.75^{\circ}$ spectrometer are:

- Momentum and energy resolution (in E/p cut), 2.5\%

- Hodoscope dead time, $\sim 2 \%$

- Algorithm (cuts), 2\%

- Hodoscope random coincidence, $\sim 1 \%$

The tracking resolutions were also determined by the Monte Carlo. The time resolution was $0.25-0.3 \mathrm{~ns}$. The resolution of the reconstructed momenta and scattering angles in the bend plane $(\sigma(\phi))$, and in the non-bend plane 


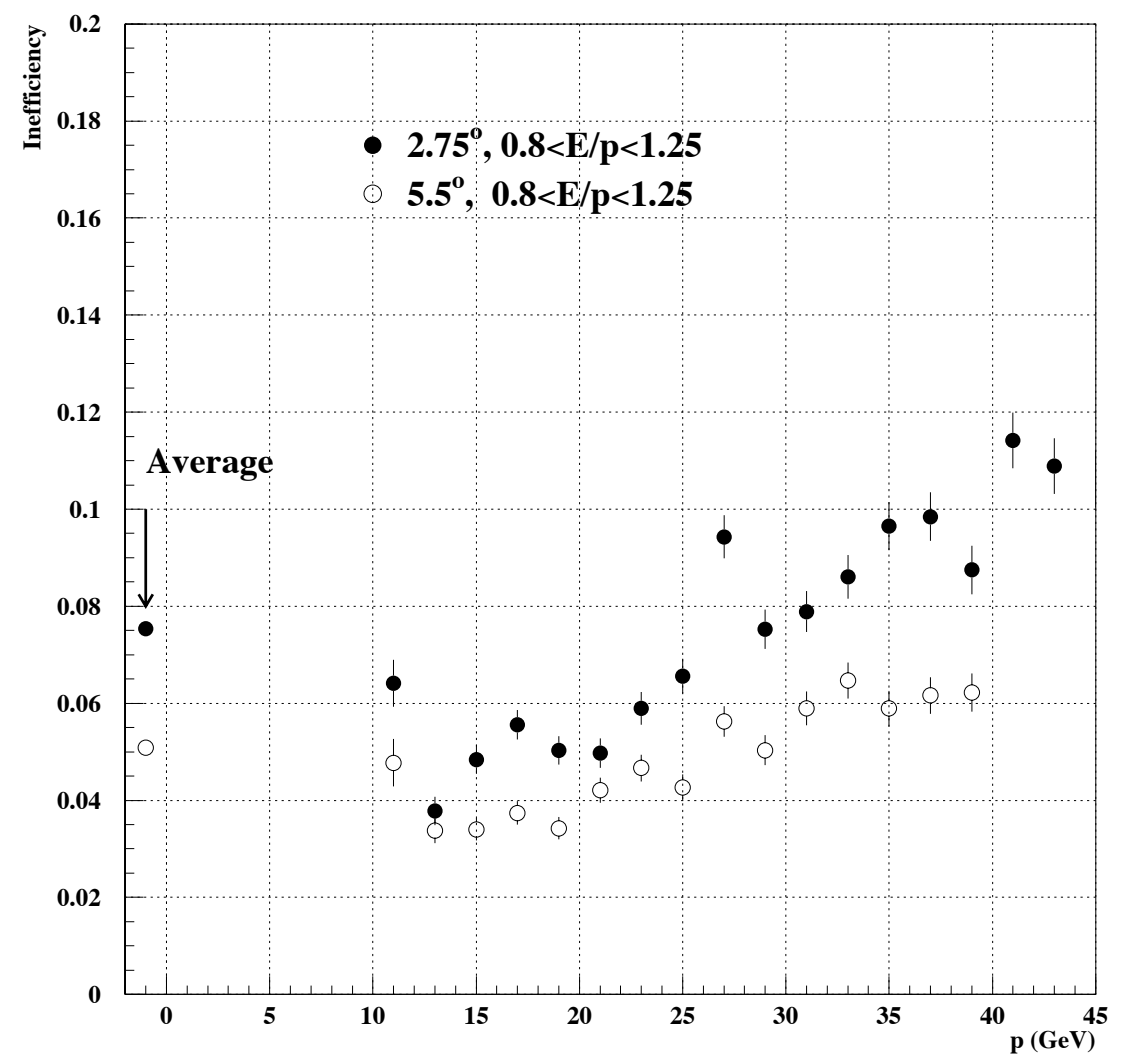

Figure 3.8: Tracking inefficiencies for two spectrometers determined by the Monte Carlo method.

$(\sigma(\theta))$ are shown in fig. 3.9. The parametrization of the momentum and angular resolutions is given by:

$$
\begin{aligned}
\frac{\sigma(p)}{p} & =\sqrt{\frac{0.013}{(\tilde{p}-7.58)^{2}}+\left(0.93 \cdot 10^{-3} \tilde{p}\right)^{2}}, \quad 2.75^{\circ}, \text { pre-Picard } \\
\frac{\sigma(p)}{p} & =\sqrt{0.011^{2}+\left(0.81 \cdot 10^{-3} \tilde{p}\right)^{2}}, 2.75^{\circ}, \text { Picard } \\
\frac{\sigma(p)}{p} & =\sqrt{0.015^{2}+\left(1.01 \cdot 10^{-3} \tilde{p}\right)^{2}}, 5.5^{\circ} \\
\sigma(\phi) & =\frac{0.037}{(\tilde{p}-7.58)^{2}}+0.194 \cdot 10^{-3}, 2.75^{\circ}, \text { pre-Picard }
\end{aligned}
$$



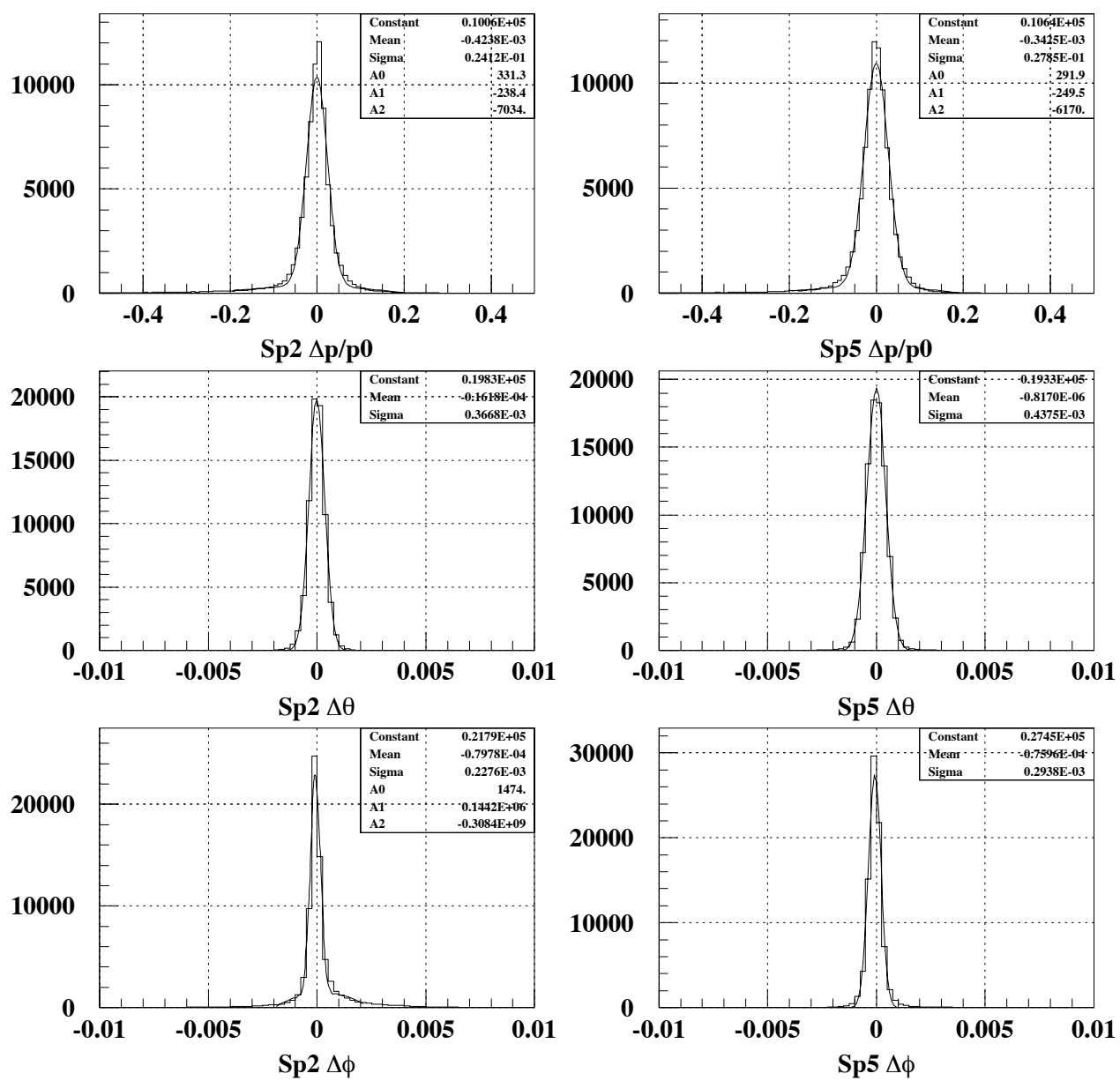

Figure 3.9: Average momentum (top) and angular resolution in the bend (middle) and non-bend (bottom) planes of the $2.75^{\circ}$ (left) and $5.5^{\circ}$ (right) spectrometers.

$$
\sigma(\phi)=\frac{0.002}{(\tilde{p}-7.58)^{0.71}}, \quad 2.75^{\circ}, \text { Picard }
$$

where $\tilde{p}=\frac{20}{p_{c}} p$, and $p_{c}$ is the central momentum of the spectrometer (all momenta are in $\mathrm{GeV})$. The angular resolution in the non-bend plane $(\sigma(\theta))$ is almost momentum independent. 


\subsection{Event analysis: Run selection and elec- tron identification}

There were 1467 polarized ${ }^{3}$ He runs written on tape during the experiment. Each run had to satisfy several criteria before it was included in the asymmetry analysis. After the selection 956 runs remained.

\subsubsection{Run selection}

Some of the runs written on tape were marked as "bad" in the log-book by the shift crew. The listed reasons included test runs with non-standard beam, target, or spectrometer configurations, frequent beam trips, poor beam quality, runs affected by hardware, DAQ or control software failures. Those runs were removed from the analysis. We required that the run be longer than 30,000 spills. In comparison, the typical "normal" run consisted of 200,000 spills. A total of 270 runs were eliminated that way from the sample.

The target polarization had to be higher than $25 \%$. The smaller polarization was usually during the spin-up of the target or special target tests and was unstable. This cut eliminated 190 runs and had negligible impact on the statistics of the results.

The measured raw asymmetries in our experiment were on the order of $10^{-3}-10^{-4}$. The measured rate dependence was smaller than $10 \%$. To keep the systematic asymmetry bias below $10^{-4}$ for every run, we insisted that beam charge asymmetry $\left|Q_{L}-Q_{R}\right| /\left|Q_{L}+Q_{R}\right|$ (the index $L$ refers to the 
"left" while $R$ to the "right" electrons) to be smaller than $5 \times 10^{-4}$. A total of 34 runs did not satisfy this condition.

The spectrometer rate was dependent on the position of the beam at the target, caused by the variation of the target cell window thickness [112]. To again keep the raw asymmetry bias below $10^{-4}$, we discarded 33 runs that had larger position fluctuations than $\left|\left\langle x_{L}\right\rangle-\left\langle x_{R}\right\rangle\right| \leq 0.004 \mathrm{~mm}$ and $\left|\left\langle y_{L}\right\rangle-\left\langle y_{R}\right\rangle\right| \leq 0.005 \mathrm{~mm}$.

Finally, as described in section 2.3.5 (page 51), if the disagreement between five monitors of the beam helicity polarization bit exceeded $5 \times 10^{-4}$, the run was rejected from the analysis. There were 18 runs with a bit comparison failure greater than $10^{-3}[73]$. Another 35 runs with the Veto signal in error [73] were included in analysis, and the polarization state was determined from the other four measurements.

\subsubsection{Event selection, electron id and kinematics}

Before the spill was analyzed for the content of electrons, it was subject to several beam quality cuts [113]. There was a

- Beam charge - absolute

requirement of $0.5 \leq Q_{\text {beam }} \leq 12$ in $10^{10}$ electrons/spill that mainly rejected the "witness" pulses used for tuning of the accelerator. Following this there was a

- Beam polarization bit match 
of all four hardware signals, with the exclusion of the above mentioned 35 runs where all but Veto signals were compared. Then there were $4 \sigma$ sliding cuts for the following quantities:

- Beam charge,

- Good spill ADC monitor,

- Bad spill ADC monitor,

- Beam width at the wire array in $x$,

- Beam width at the wire array in $y$,

- Beam position at the wire array in $x$,

- Beam position at the wire array in $y$,

- Beam position at the TWBPM in $x$,

- Beam position at the TWBPM in $y$.

The mean value and RMS of distributions for the " $4 \sigma$ " cuts were calculated every 1056 (32 cycles of 33) spills of the random number generator for the bit polarization. The values were used for the next 1056 spills and then changed in accordance with the new computation. That allowed to follow the slow changes of the beam characteristics while rejecting the instantaneous perturbations. The most important cuts were the beam charge and the position at the wire array in $y$ needed to control the rate dependence, and the cut on the bad spill ADC monitor for the overall beam quality. 
The electron cuts were based on the systematic studies of 52 electron and 14 pion definitions [91]. The definition used for the asymmetry computations was optimized for maximum efficiency (statistics), minimum pion contamination and small rate dependence. The implementation consisted of the following cuts:

- Class 1 track with both Cherenkovs in coincidence,

- Acceptance cut passed,

- $8 \mathrm{GeV}<\mathrm{p}<48.3 \mathrm{GeV}$,

- $Q^{2} \geq 1 \mathrm{GeV}^{2}, W^{2} \geq 5 \mathrm{GeV}^{2}$,

- One of the Cherenkov signals greater than 2.5 photoelectrons,

- $E>7.5 \mathrm{GeV}$,

- $E / p \geq 0.8$,

- Shower shape $\chi \leq 0.045$ for $p<30 \mathrm{GeV}$,

- No DAQ failures for the spill in a given spectrometer

Some of the cuts are illustrated in fig. 3.10. The class 1 track required an association with a shower cluster, at least one Cherenkov signal, four (three) front and three rear hodoscope hits in the $2.75^{\circ}\left(5.5^{\circ}\right)$ spectrometer. The acceptance cut was defined by the horizontal $\theta$ and vertical $\phi$ scattering angles at the target,

$$
-\theta_{\max } \leq \theta \leq \theta_{\max }, \quad \phi_{\min } \leq \phi \leq \phi_{\max }
$$




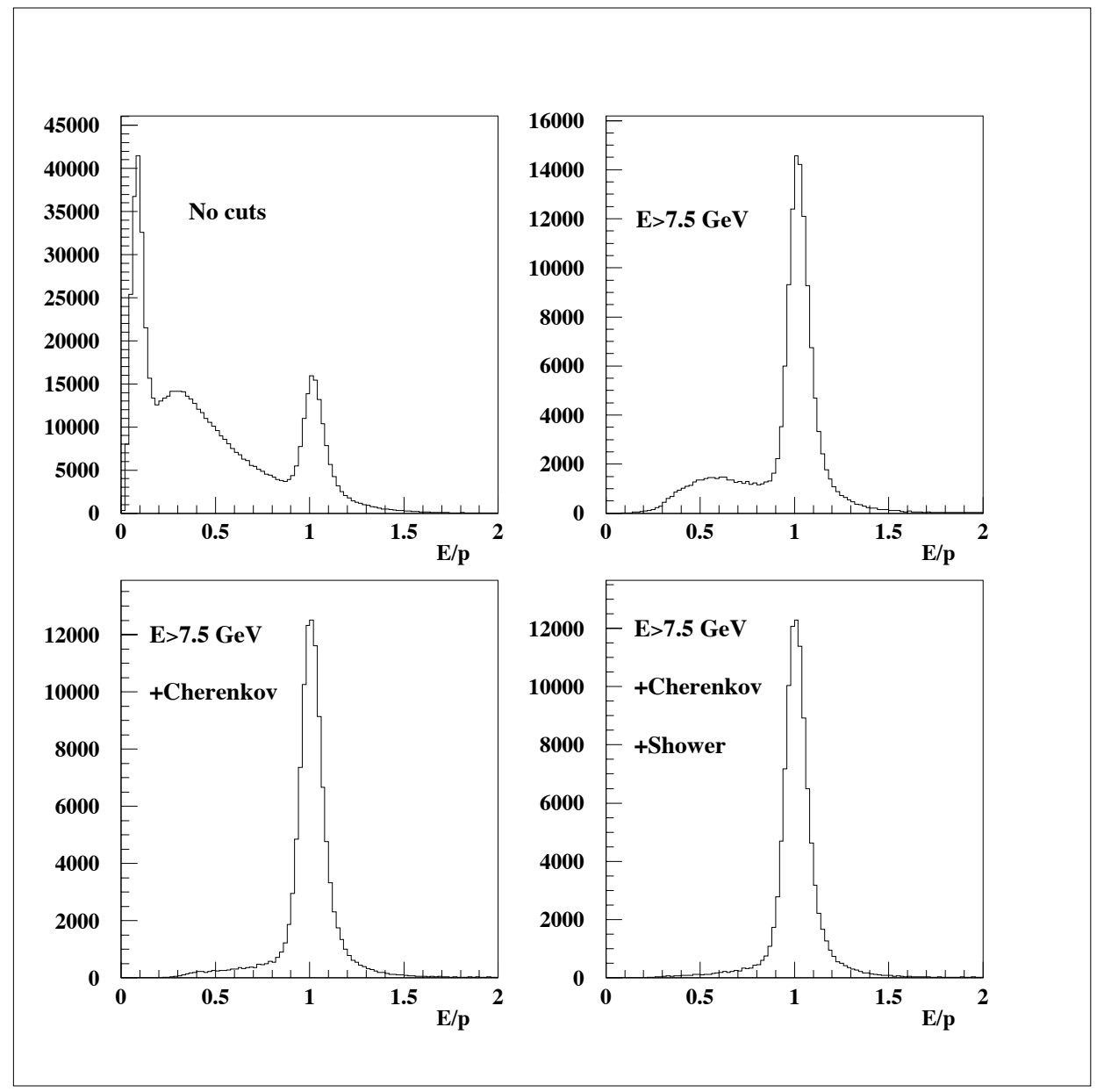

Figure 3.10: Cuts influence on $E / p$ distribution.

where $\theta_{\max }=0.006(0.013) \mathrm{rad}, \phi_{\min }=-0.031(-0.028) \mathrm{rad}$ and $\phi_{\max }=$ $0.005(0.01) \mathrm{rad}$ for $2.75^{\circ}\left(5.5^{\circ}\right)$ spectrometer.

The kinematics of an event was determined from the tracking. The scattering angle of an electron is

$$
\vartheta=\sqrt{\left(\theta_{\circ}+\theta\right)^{2}+\phi^{2}}
$$


where $\theta_{\circ}=+2.75^{\circ}$ or $+5.5^{\circ}$. The track momentum $p$ determined the scattered energy $E^{\prime}$. The kinematic variables are

$$
\begin{aligned}
Q^{2} & =4 E E^{\prime} \sin ^{2}(\vartheta / 2), \\
x & =\frac{Q^{2}}{2 M\left(E-E^{\prime}\right)}, \\
W^{2} & =M^{2}+Q^{2} \frac{(1-x)}{x},
\end{aligned}
$$

where $E$ is the beam energy and $M$ is the neutron mass.

\subsection{Asymmetry analysis}

The summary files obtained for each run from DSTs contained the beam charge $Q_{b}$, the average $Q^{2}$ for a given beam-target spin configuration, and the number $N$ of electrons in an $x$ bin. The values of $x$ and $Q^{2}$ were computed from the track reconstructed angle and momentum of the scattered electron. The information was used for computing the raw asymmetries $A_{\|}^{\text {raw }}$ and $A_{\perp}^{\text {raw }}$ :

$$
A_{\|}^{r a w}(x)=\frac{\left(N / Q_{b}\right)^{\downarrow \Uparrow}-\left(N / Q_{b}\right)^{\uparrow \uparrow}}{\left(N / Q_{b}\right)^{\downarrow \uparrow}+\left(N / Q_{b}\right)^{\uparrow \Uparrow}}
$$

and a similar formula for $A_{\perp}^{\text {raw }}(x)$, see eq. (1.11), (1.12) (section 1.2, page 7). At the same time the average value of $Q^{2}$ for a given $x$ was obtained. The statistical error for the asymmetries is given by

$$
\sigma\left(A_{\|}^{\text {raw }}\right)=\frac{1}{\sqrt{\left(N / Q_{b}\right)^{\downarrow \Uparrow}+\left(N / Q_{b}\right)^{\uparrow \Uparrow}}}\left(1+\mathcal{O}\left[\left(A_{\|}^{\text {raw }}\right)^{2}\right]\right),
$$

where $\mathcal{O}\left[\left(A_{\|}^{\text {raw }}\right)^{2}\right]$ can be neglected since $A_{\|}^{\text {raw }} \sim 10^{-3}$. 
Several corrections have to be applied before the raw asymmetries can be used to calculate the virtual photon-nucleon asymmetries and spin dependent structure functions. The data sample is slightly contaminated in two ways. Some of the electrons are misidentified pions. In addition, a small portion of electrons comes from charge symmetric processes, such as $\pi^{\circ} \rightarrow e^{+} e^{-} \gamma, \gamma \rightarrow e^{+} e^{-}$etc., rather than from the scattering. We will call both contaminations a background and denote by $A_{e}^{\text {raw }}$ the raw asymmetry with the background subtracted. The reconstruction efficiency depends somewhat on the electron rate. Different fractions of events are detected for one helicity configuration in comparison to the other. The correction $\Delta A^{\text {rate }}$ due to that difference will be called the rate dependent correction. The precision of our data requires taking into account a small addition to the asymmetry coming from a parity-violating interference of $\gamma$ and $Z^{\circ}$ exchange amplitudes, in terms of the electro-weak correction, $A^{E W}$. Next we need to remember the dilution in terms of the beam polarization $P_{b}$, target polarization $P_{t}$ and the dilution factor $f$ (see sec. 3.10, page 154). Finally we need to correct for the fact that all our considerations are limited to the tree level DIS diagram, while the measurement includes all order radiative processes and tails from elastic, quasi-elastic and inelastic scatterings. We thus apply a radiative correction, $\Delta A^{R C}$. Combining all the corrections, the physics asymmetry, $A^{\text {phys }}$, can be calculated from the formula:

$$
A^{\text {phys }}=\frac{A_{e}^{\text {raw }}+\Delta A^{\text {rate }}-P_{b} A^{E W}}{P_{b} P_{t} f}+\Delta A^{R C} .
$$

The following sections will describe the above corrections. 


\subsection{Background subtraction}

The events passing our cuts and used to form the asymmetry are not a pure sample of deep inelastically scattered electrons. Some of them are misidentified negatively charged hadrons (mainly pions) or muons. We will refer to them as the pion background and denote their fraction with respect to all electrons by $\pi / e$ and their asymmetry by $A_{\pi}$. The other source of the background consists of electrons originating in the charge symmetric processes, e.g. $\pi^{\circ} \rightarrow \gamma e^{+} e^{-}, \gamma \rightarrow e^{+} e^{-}$, etc. There is an $e^{+} / e$ fraction of those events, and they have an asymmetry $A_{e^{+}}$. Any spin dependence of the background processes will modify our $A^{\text {raw }}$ measurement directly. The asymmetry $A_{e}^{\text {raw }}$ due to DIS electrons can be calculated from the following decomposition of the measured asymmetry:

$$
A^{\text {raw }}=\left(1-\pi / e-e^{+} / e\right) A_{e}^{r a w}+(\pi / e) A_{\pi}+\left(e^{+} / e\right) A_{e^{+}} .
$$

For small background fractions, neglecting the quadratic terms, the DIS asymmetry can be written in a form:

$$
A_{e}^{\text {raw }}=\frac{1}{1-e^{+} / e}\left[\frac{A^{\text {raw }}-(\pi / e) A_{\pi}}{1-\pi / e}-\left(e^{+} / e\right) A_{e^{+}}\right]
$$

with an obvious interpretation: From the measured asymmetry the pion background is subtracted and the result is corrected for the dilution, next the positron background is subtracted and again the outcome is corrected for the dilution due to that contamination. 


\subsubsection{Pion background}

The pion contamination ratio $\pi / e$ was determined from the $E / p$ distribution in the following way [91]. The $E / p$ spectrum is obtained for all the events passing our cuts, see "Electrons" in fig. 3.11. At low $E / p$ values it is

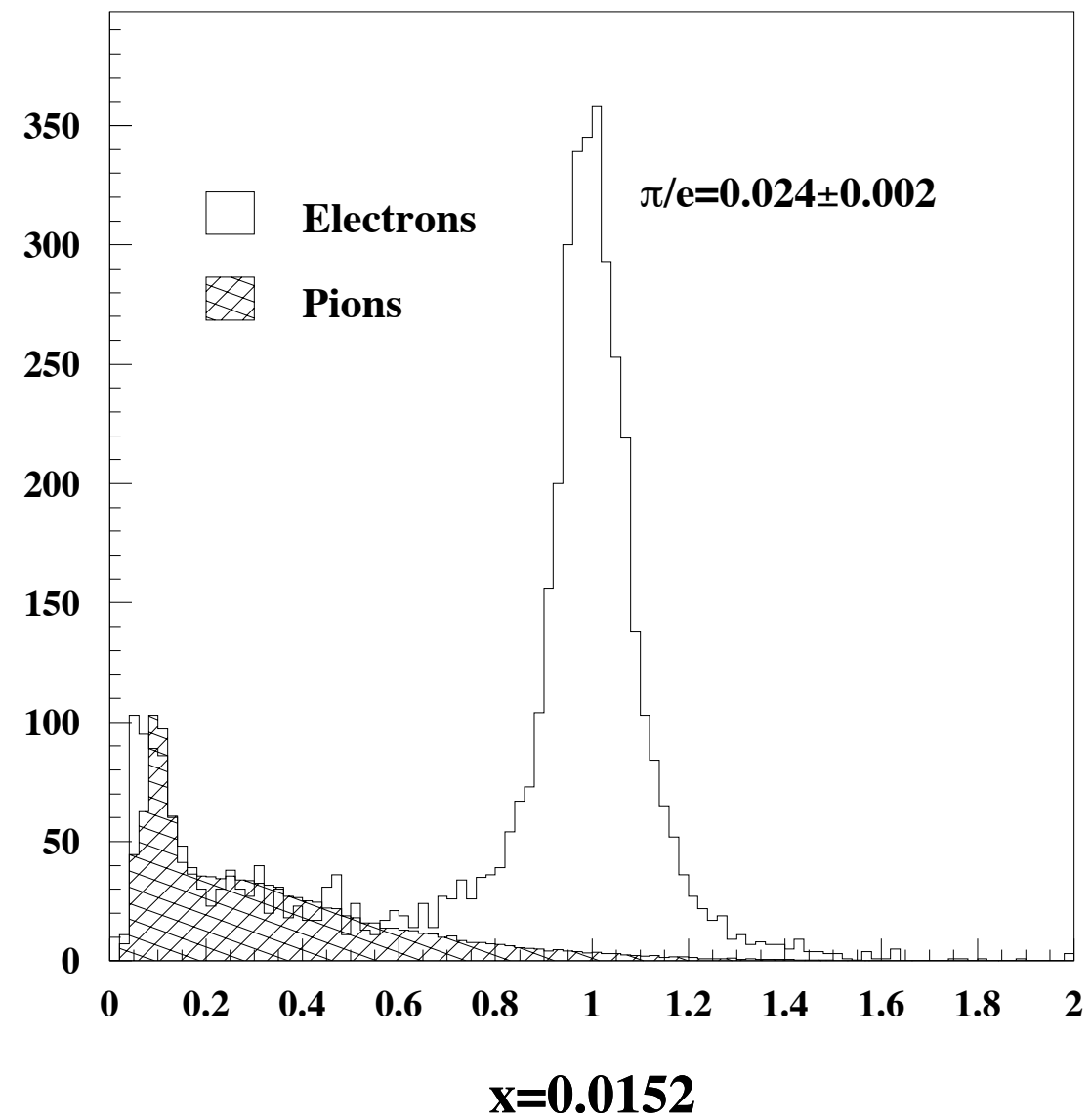

Figure 3.11: Pion contamination in the $x=0.0152$ bin.

dominated by pions that do not shower. The electrons are defined as those events for which $E / p \geq 0.8$. Next the pion $E / p$ distribution is constructed. The pions are well identified by a veto in both Cherenkov detectors, and they 
peak at low $E / p$ values. Matching the spectra at low $E / p$ determines the pion tail in the electron distribution of $E / p \geq 0.8$ and thus establishes the $\pi / e$ ratio. The pion contamination for the worst case is shown in fig. 3.12. The average contamination at the lowest $x$ was $2.8 \%$ and diminished with

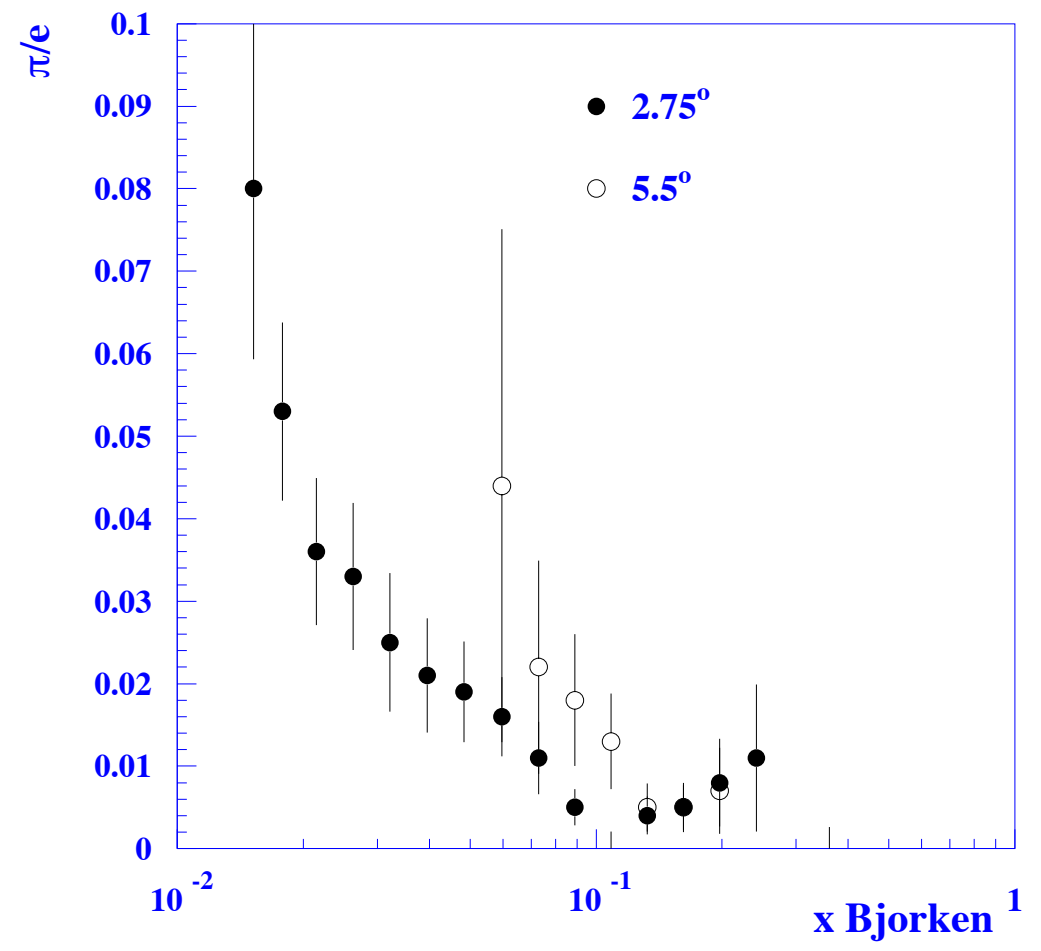

Figure 3.12: Pion contamination.

increasing $x$. The error in $\pi / e$ was estimated from the spread of different methods [114] used to determine that ratio and is taken to be $50 \%$ of the $\pi / e$ value. The measured pion asymmetry was three times smaller than the electron asymmetry, see fig. 3.13 . 


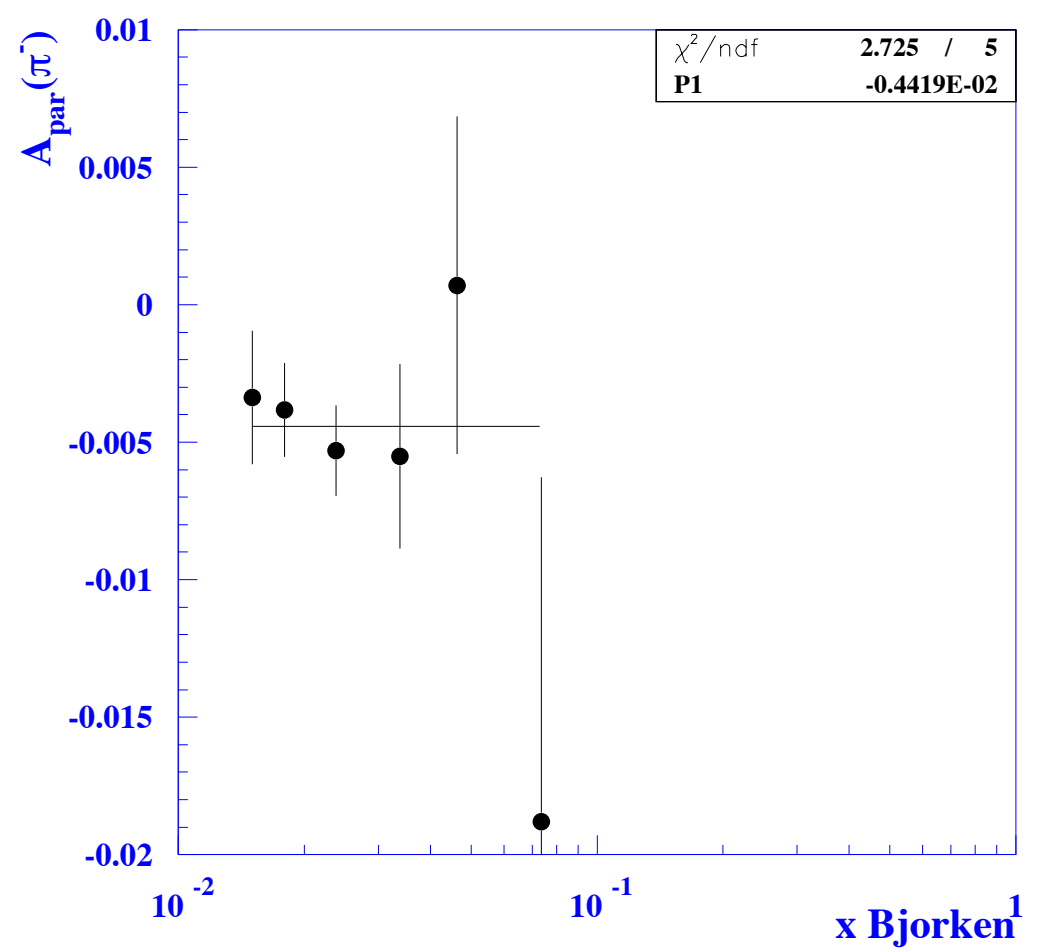

Figure 3.13: Pion asymmetry.

\subsubsection{Charge symmetric background}

The event rate of electrons coming from charge symmetric processes was determined by reversing the polarities of the spectrometer magnets. The measured positron component of the background was assumed to be equal to the electron rate coming from the charge symmetric background. There were 81 positron runs with the longitudinally polarized target Picard. The extracted "positron" contamination fraction $e^{+} / e$ and the positron asymmetry $A_{e^{+}}[91]$ are shown in fig. 3.14 and 3.15 respectively. The positron rate was also measured with the different empty and full reference cells, and a 


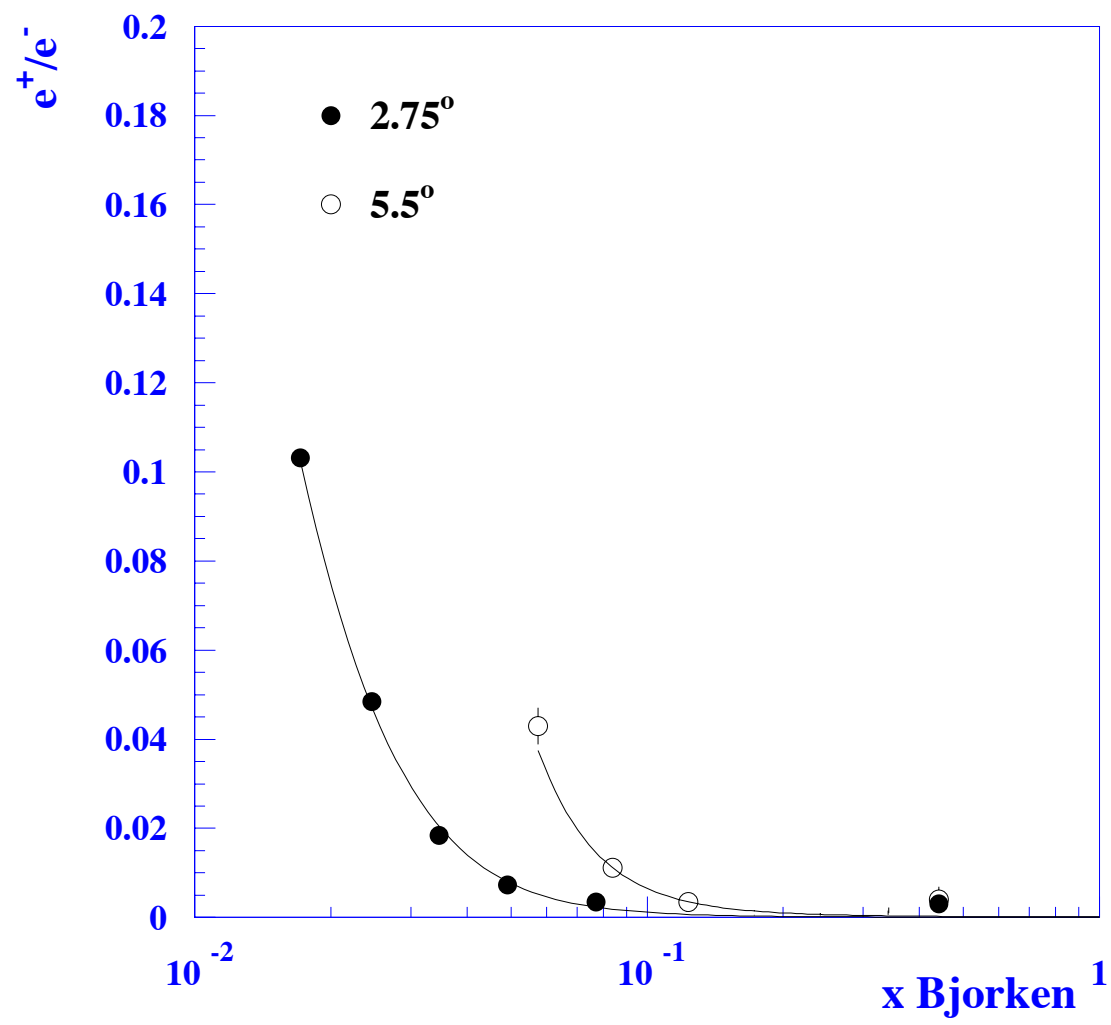

Figure 3.14: Positron contamination.

variation of around $20 \%$ was observed. Since the positron runs were taken for one target cell only, we assign $20 \%$ systematic error to the $e^{+} / e$ ratio.

The positron asymmetry was found to be consistent with zero. For the asymmetry correction we assume $A_{e^{+}}=0$ and use the (quite large) statistical error of the measurement as the systematic uncertainty in $g_{1}^{n}$ due to the charge symmetric background. This uncertainty dominates the systematic error in the lowest $x=0.017$ bin, where the fraction $e^{+} / e$ is the largest. The error could be significantly decreased if there were theoretical guidance 


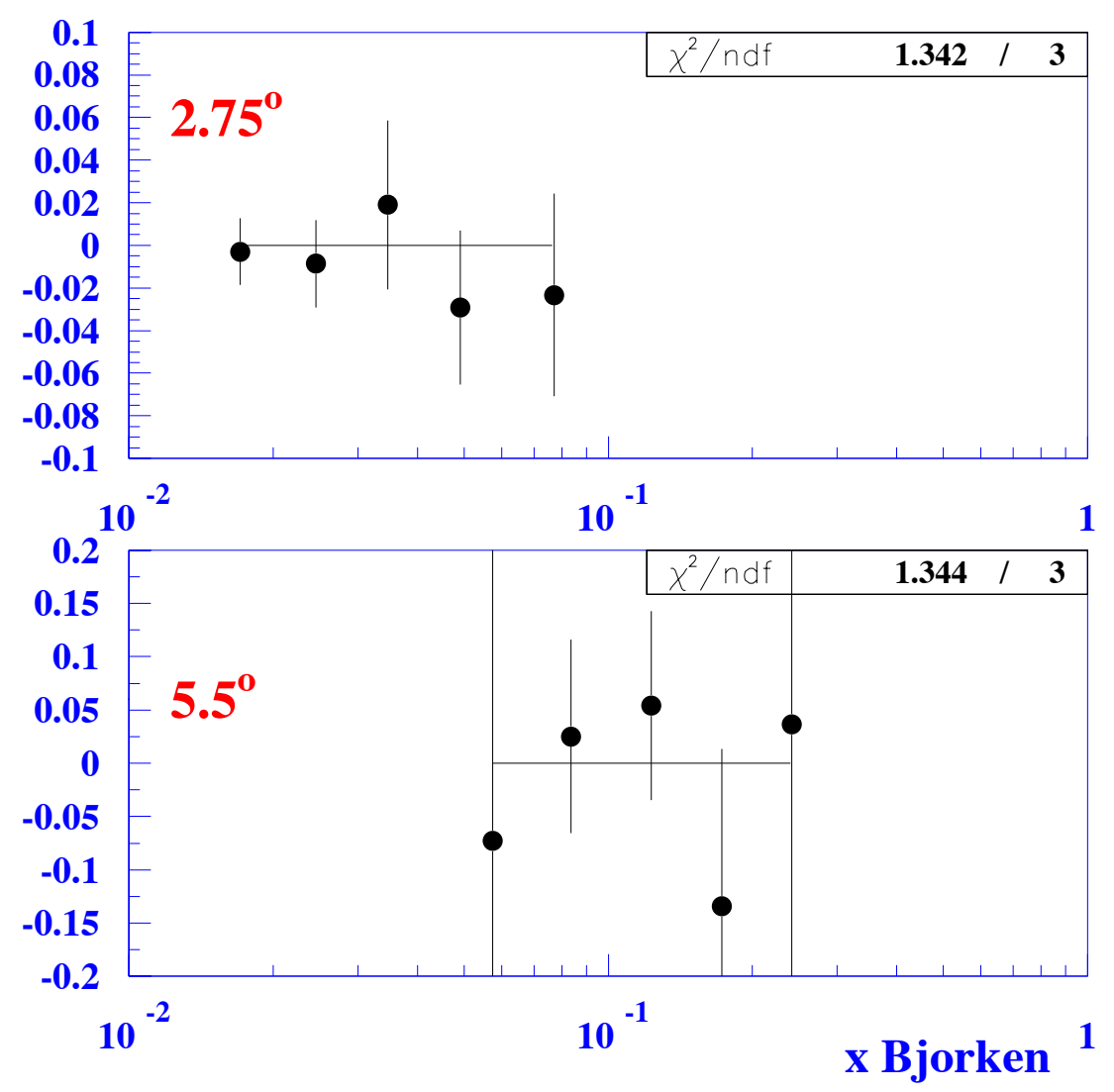

Figure 3.15: Positron asymmetry.

regarding the charge symmetric background processes. If, for instance, the $x$ dependence of the asymmetry is weak, then we could average the positron data over $x$ and reduce the systematic error in the lowest $x$ by $30 \%$. Or, if the dominant mechanism for the $e^{+} e^{-}$creation is $\pi^{\circ}$ photo-production, then it seems likely, on the basis of isospin symmetry, that the positron asymmetry is bound by the $\pi^{-}$and $\pi^{+}$asymmetries, which were measured much more accurately. That would decrease the systematic error in the lowest $x$ by $40 \%$. 


\subsection{Rate dependence}

The measured raw asymmetries, as given by eq. (1.11), (1.12) (page 7), are calculated from the observed detector rates in the spectrometers. Before the rate asymmetries are related to the cross section asymmetries (cf. eq (1.15), (1.16)), the observed rates should be converted to true rates via spectrometer acceptance and detector efficiencies. The target polarization was reversed six times during our experiment. The beam helicity, however, was randomized every pulse. Such rapid helicity changes allow one to neglect the slow drifts in spectrometer acceptance and slow changes in detector efficiencies. The acceptance and efficiencies cancel out in eq. (1.11), (1.12).

The efficiencies, however, could be affected by instantaneous changes, and one has to correct for these, despite the frequent helicity flips. Suppose that the efficiency $\varepsilon$ depends linearly on the rates $N$ in the detector,

$$
\varepsilon(N)=\varepsilon_{0}(1-\beta N)
$$

where $\varepsilon_{0}$ is the rate independent efficiency and $\beta$ is some constant characterizing rate dependence. The number of reconstructed electrons $N_{e}$ is a function of the detector rate,

$$
N_{e}(N)=N_{\circ} \varepsilon(N)=N_{\circ} \varepsilon_{\circ}(1-\beta N)
$$

Here $N_{\circ}$ is the true number of electrons in the spectrometer. The detector rates $N^{L, R}$ for the left and right electrons are different. Thus the measured asymmetry

$$
A_{e} \equiv \frac{N_{e}^{L}\left(N^{L}\right)-N_{e}^{R}\left(N^{R}\right)}{N_{e}^{L}\left(N^{L}\right)+N_{e}^{R}\left(N^{R}\right)} \approx A_{\circ}+\beta\langle N\rangle A,
$$


is biased proportionally to the rate dependence $\beta$ and the detector asymmetry

$$
A=\frac{N^{L}-N^{R}}{N^{L}+N^{R}}
$$

Here

$$
A_{\circ}=\frac{N_{\circ}^{L}-N_{\circ}^{R}}{N_{\circ}^{L}+N_{\circ}^{R}}
$$

is the true electron asymmetry, and $\langle N\rangle=\frac{1}{2}\left(N^{L}+N^{R}\right)$ is the average detector rate. The higher order terms in $A_{\circ}$ and $\beta$ on the right hand side of eq. (3.13) have been neglected. Suppose that in addition to the reconstruction of events with the detector rates $N$, we could reconstruct events at the doubled rate $2 N$. That would determine the ratio

$$
\alpha=\frac{N_{e}(N)-N_{e}(2 N)}{N_{e}} \approx \beta N,
$$

so that the true asymmetry could be computed as,

$$
A_{\circ}=A_{e}+\alpha A \text {. }
$$

The above expression is easily generalized to several detectors:

$$
A_{\circ}=A_{e}+\alpha_{\text {cher }} A^{\text {cher }}+\alpha_{\text {hodo }} A^{\text {hodo }}+\alpha_{\text {shw }} A^{\text {shw }},
$$

so the correction $\Delta A^{\text {rate }} \equiv A_{\circ}-A_{e}$ due to the rate dependence is,

$$
\Delta A^{\text {rate }}=\alpha_{\text {cher }} A^{\text {cher }}+\alpha_{\text {hodo }} A^{\text {hodo }}+\alpha_{\text {shw }} A^{\text {shw }},
$$

where $A^{\text {cher }}$ is the Cherenkov counters asymmetry, $\alpha_{\text {cher }}$ is the normalized change of rates, eq. (3.14), for the double rate in the Cherenkovs only, and similarly for the hodoscopes (index hodo), and shower (index shw). 


\subsubsection{Pulse fiction method}

The studies of the rate dependence were performed with a "pulse fiction" $\operatorname{method}^{1}$.

After standard analysis the two consecutive spills were merged, simulating the doubled rate. The fused spill was re-analyzed and the outcome was compared to the single rate results.

\section{Merging the spills}

The spills were merged on the level of raw data as a hardware response to the double rate. The shower counter ADCs were combined in a straightforward manner. An ADC integrated the signals from the whole spill, and the result is directly proportional to the charge. The merged response was thus the channel by channel sum of single rate responses, as illustrated in fig. 3.16. The FADCs were fused similarly: simple addition of the signals in the same time interval with several adjustments. The signals had to be shifted in time with respect to each other so the TDC synchronization algorithm would work properly. The summed baseline was lowered to the level of a single rate line. The signals were added to the maximum level of 252 channels of FADC. Fig. 3.17 illustrates the process. In adding the TDC signals dead time was taken into account. Careful attention was paid to the overlapping signals from TDCs working in a burst mode, that gave the leading and trailing edge information, to properly simulate hardware response. The

\footnotetext{
${ }^{1}$ The similarity of the name to the title of the popular motion picture at the time should be noted.
} 


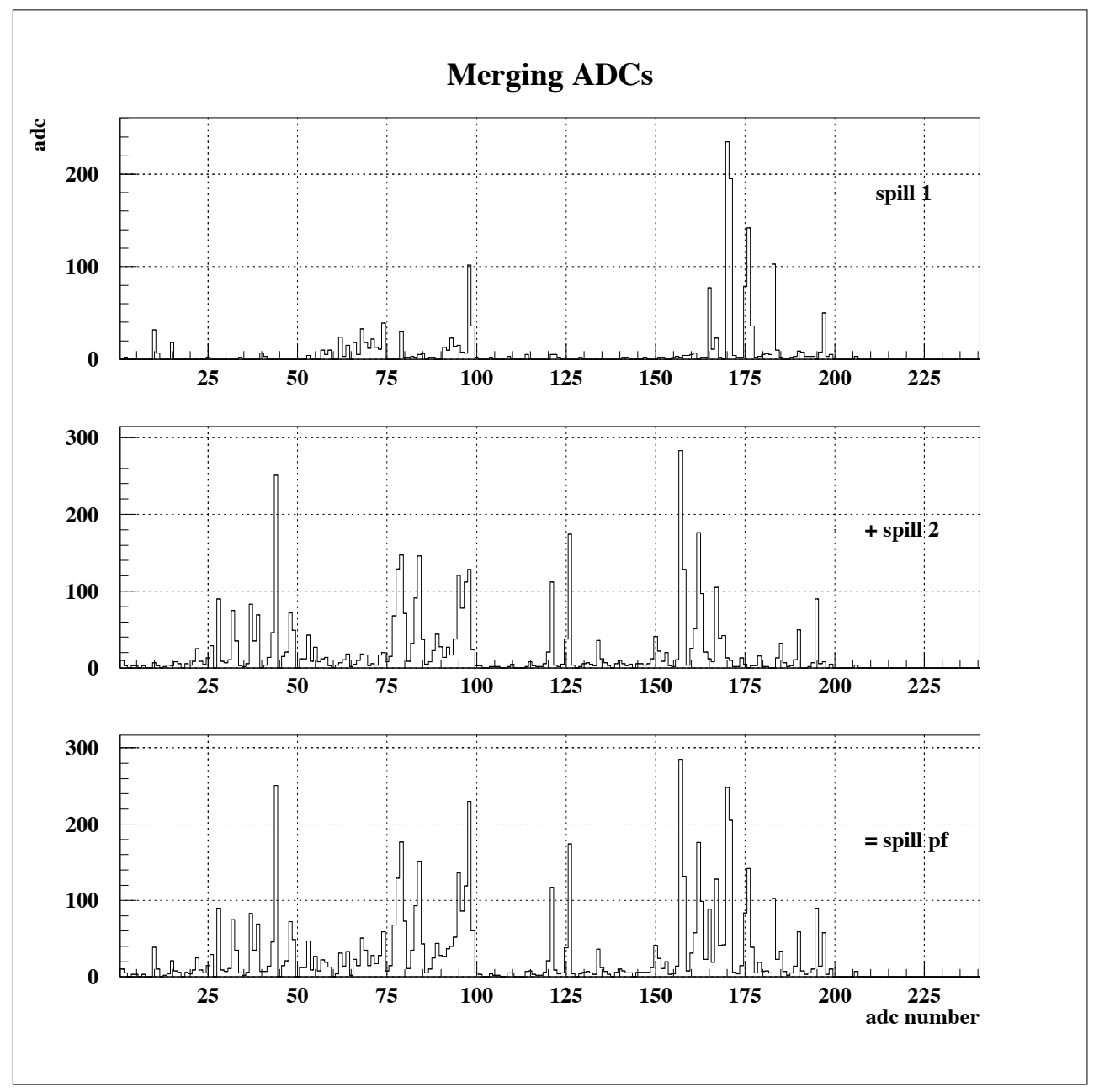

Figure 3.16: ADC signals from spill 1 and 2 merged to the double spill rate.

merging resulted in the loss of $\sim 3 \%$ hodoscope hits and $\sim 2 \%$ Cherenkov signals (with hight above 10 FADC units) due to the dead time, and in the gain of $\sim 8 \%$ shower clusters due to the overlaps (more clusters passed the minimum energy requirement). 


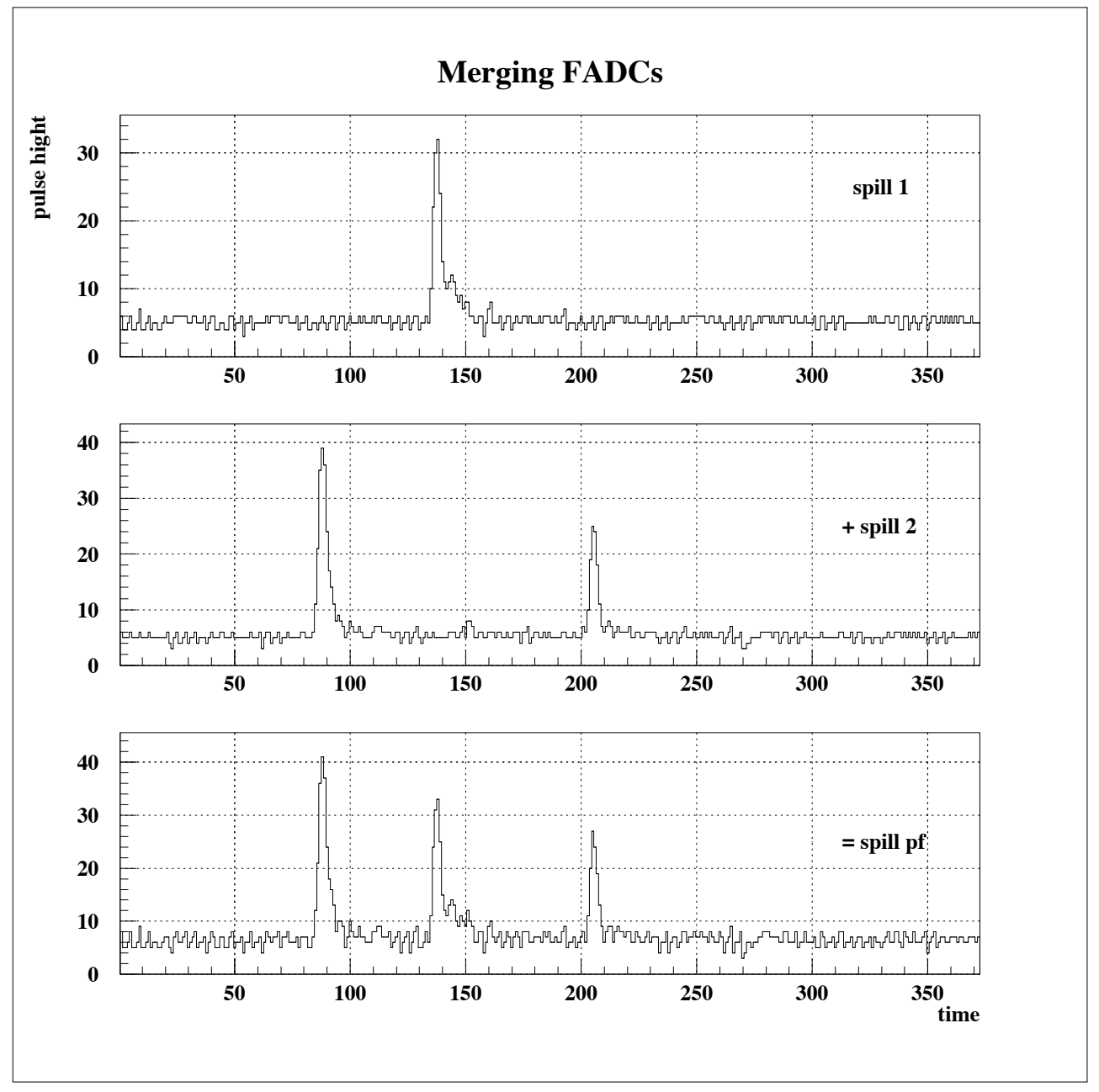

Figure 3.17: FADC signals from spill 1 and 2 merged to the double spill rate.

\section{Matching events}

To compare how the quantities associated with the track were altered by the doubled rate, the tracks from the single spills were matched with the pulse fiction tracks. The equivalent tracks had to be within $2.5 \mathrm{~ns}$ time window (cf. fig. 3.18) and within $100 \mathrm{~mm}$ from each other at the front hodoscopes and at 


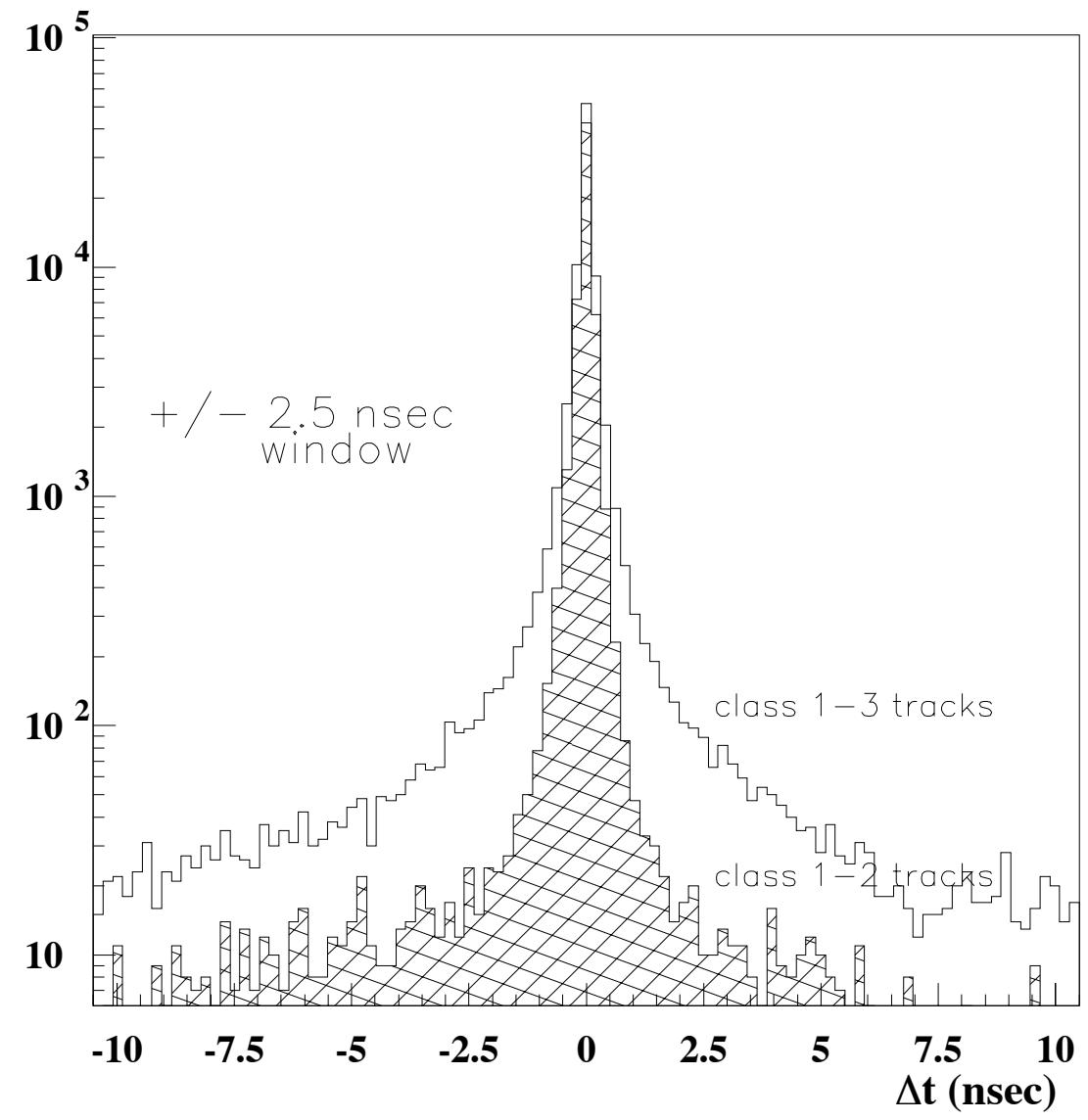

Figure 3.18: The distribution of the time difference between single and double rates tracks.

the surface of the calorimeter (cf. fig. 3.19). The matching cuts were quite loose. If there was more than one candidate for the corresponding track, the one closest in time was chosen.

\subsubsection{Momentum and energy rate dependence}

Having mated the tracks, we could investigate the energy and momentum changes with rates. The relative changes, for the standard electron defini- 


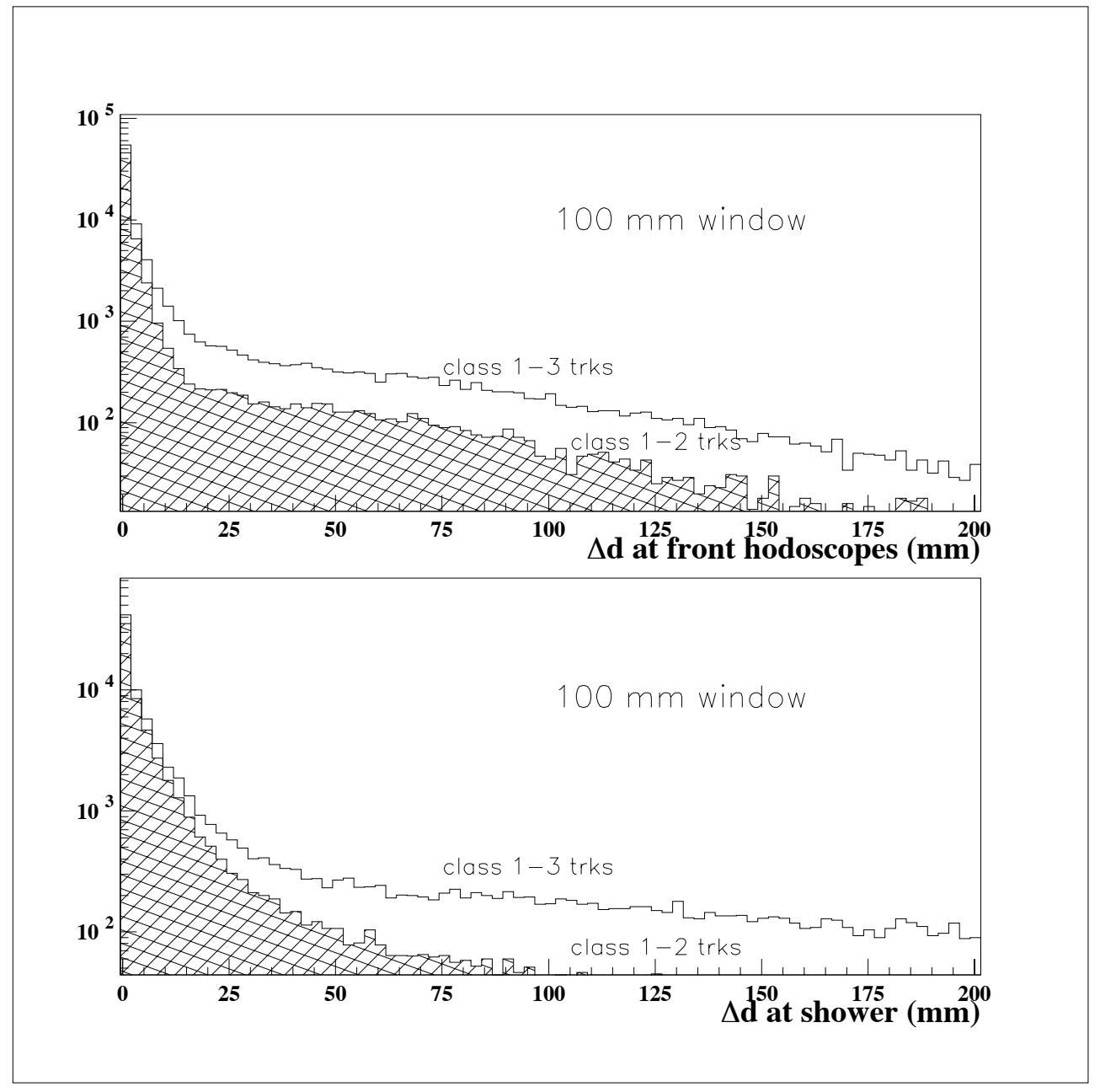

Figure 3.19: The distribution of distance between single and double rates tracks at the front hodoscopes (top) and at the shower counter (bottom).

tion, with respect to the values $E, P$, associated with the single rate tracks, $\Delta E / E \equiv\left(E-E_{\mathrm{pf}}\right) / E$ and $\Delta P / P \equiv\left(P-P_{\mathrm{pf}}\right) / P$, are shown in fig. 3.20 $\left(2.75^{\circ}\right.$, target cell Picard). The error bars represent the mean value uncertainty. The average momentum is robust against the rate changes. The 


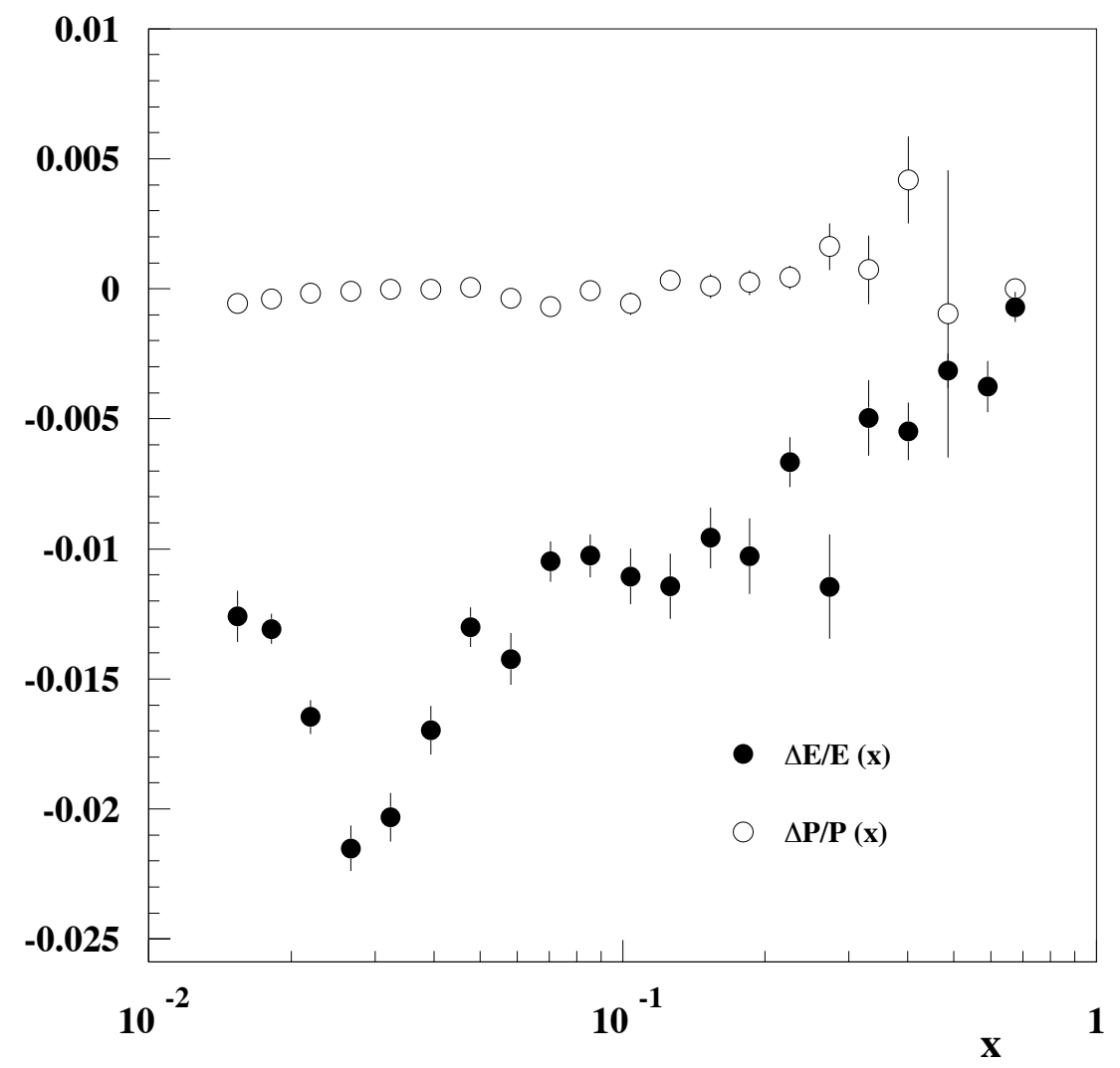

Figure 3.20: The relative momentum (open circles) and energy (closed circles) changes as a function of Bjorken $x$ in $2.75^{\circ}$ spectrometer (target cell Picard) when the rate is doubled.

energy differences are relatively strongly $x$ dependent. Especially at low values of $x$ they are sensitive to electron pileups with pions, despite the three TDC levels used. The shower pileups are weakly correlated with the rate dependence of other detectors. Thus the profile in $x$ of the changes in energy is the same for other electron definitions that have the same energy cuts as the standard definition. On the other hand, if the definition relies differently on energy, the magnitude of the $x$ dependence changes. For example, the 
definition with $E / p$ cut on both sides (i.e. $0.8 \leq E / p \leq 1.2$ ) has a $30 \%$ smaller rate dependent energy due to the rejection of the tracks with highly overestimated overlap energy. The cost is reduced electron efficiency.

The energy and momentum rate sensitivity in $2.75^{\circ}$ for all target cells is shown in fig. 3.21. Notice the stability of the momentum determination. The energy rate dependence at low $x$ for the first four targets is relatively large. It is due to the high beam current of $9 \times 10^{10}$ electrons/spill which was later lowered to $5 \times 10^{10}$ electrons/spill. The further lowering of the current to $3 \times 10^{10}$ for the target Picard had a much smaller effect. The changes in the lowest three $x$ bins for the Dave, Riker, Bob and SMC targets are due to the gradual upgrade from one to three TDC levels for the "hottest" calorimeter blocks. The situation in the $5.5^{\circ}$ spectrometer was similar. The momentum was stable; the energy rate dependence was the same as in the $2.75^{\circ}$ arm for the highest beam current and roughly 2 times smaller for lower currents (this is mainly due to the fact that the $5.5^{\circ}$ electron rates were lowered with every current change, while the $2.75^{\circ}$ electron rates were kept constant by the collimators adjustment). Based on these results, we chose track momentum $p$ rather than the calorimeter energy $E$ as the energy of the scattered electron.

\subsubsection{Detector rate dependence}

The pulse fiction method was also used to establish the detector rate dependence in terms of the coefficients $\alpha$, straight from the defining ratio of numbers of reconstructed electrons given by eq. (3.14). The analysis was performed separately for each detector system by merging only the signals 


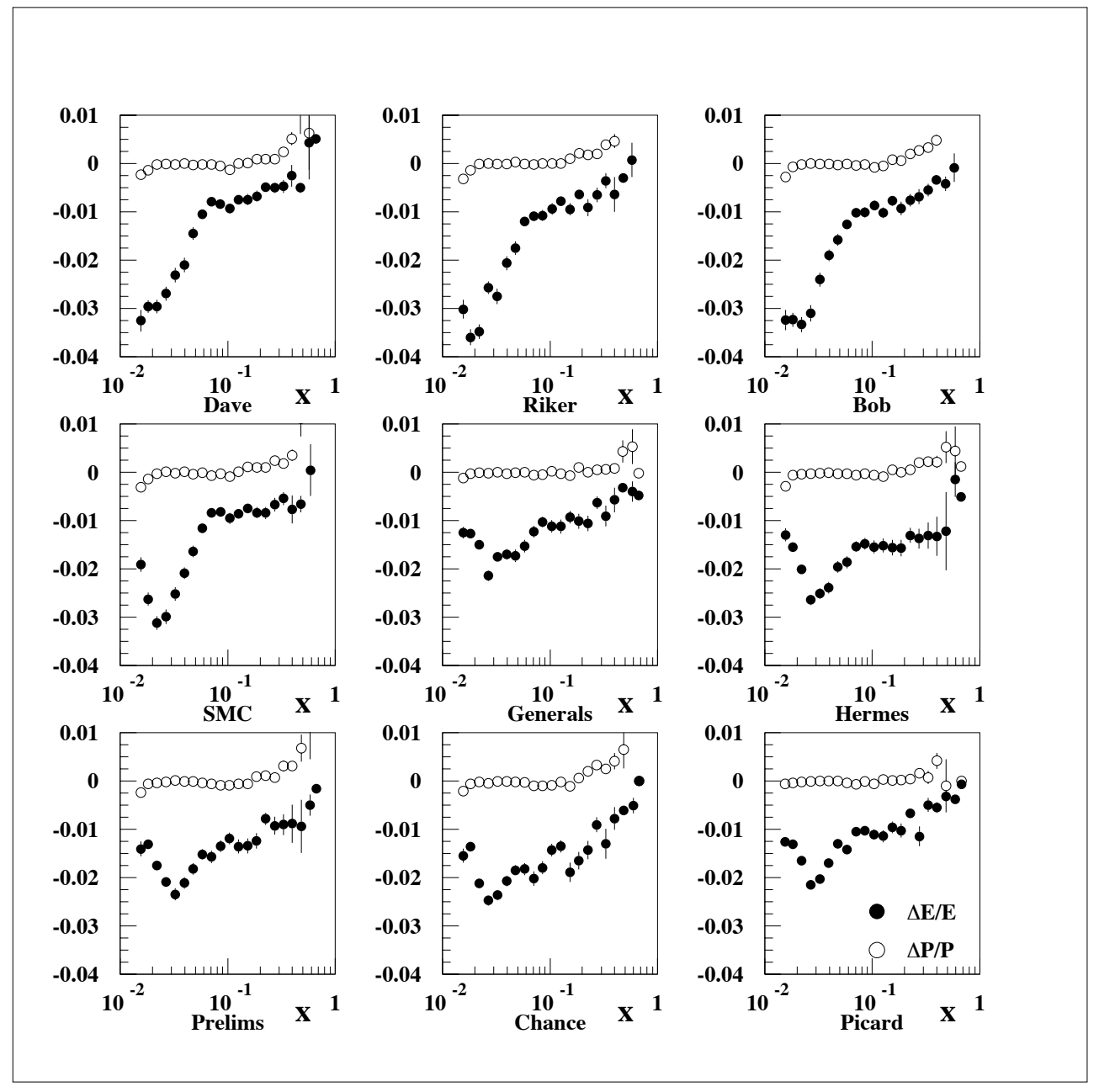

Figure 3.21: The relative momentum (open circles) and energy (closed circles) changes, when the rate is doubled, as a function of Bjorken $x$ in $2.75^{\circ}$ spectrometer for all target cells.

from a considered detector to simulate the rate doubling in this system and leaving the other ones unchanged (single rate). That allowed us to compute $\alpha_{\text {cher }}(x), \alpha_{\text {hodo }}(x)$ and $\alpha_{s h w}(x)$. Simultaneous fusion of all the signals determined $\alpha_{\text {total }}(x)$. The results for the target cell Picard, $2.75^{\circ}$ are shown in 
fig. 3.22. The hodoscopes rate dependence caused by the TDCs dead time

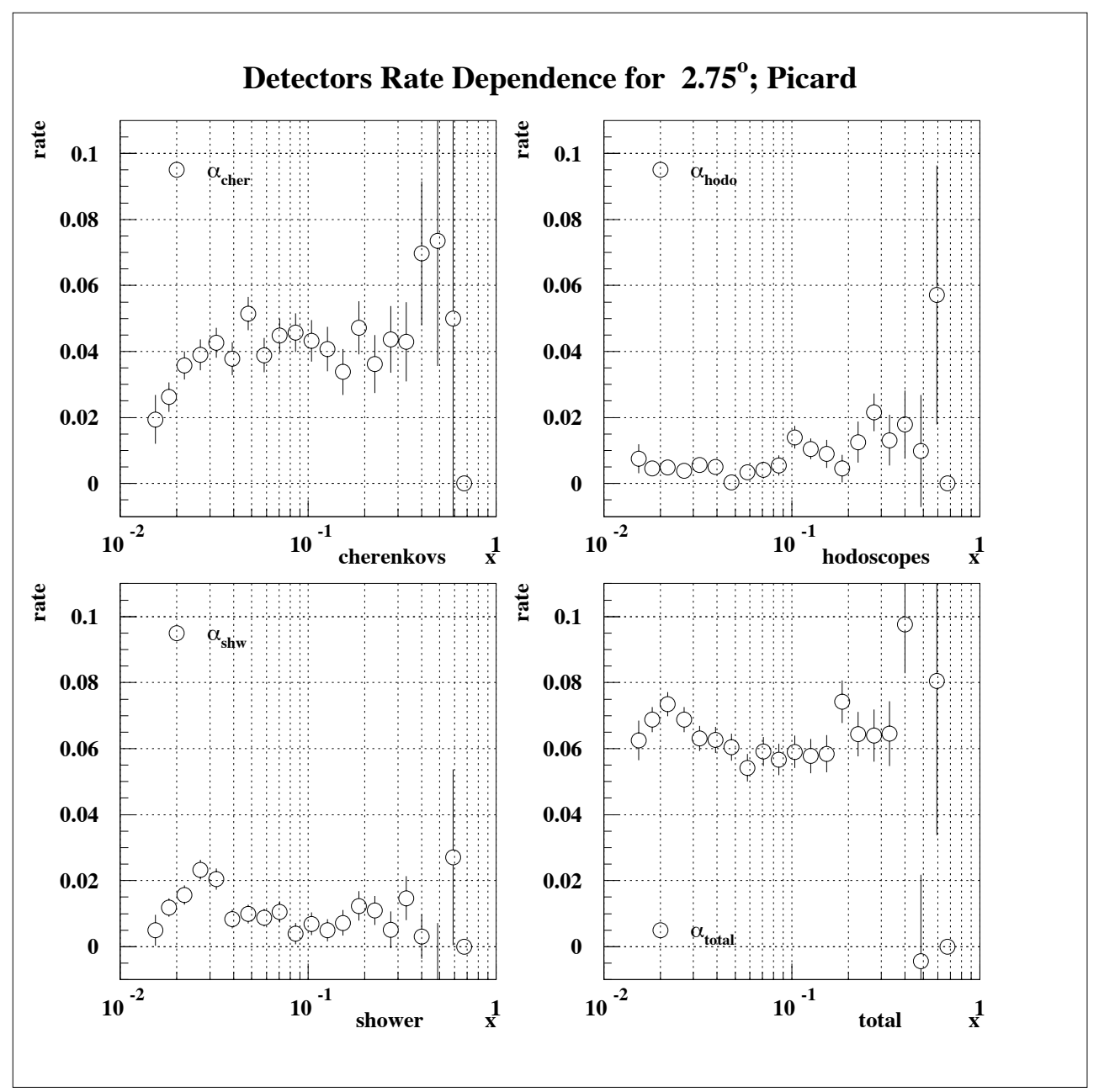

Figure 3.22: The detector rate dependence coefficients $\alpha_{c h e r}, \alpha_{\text {hodo }}, \alpha_{s h w}$ and $\alpha_{\text {total }}$ for the target cell Picard, $2.75^{\circ}$ spectrometer.

is small and roughly flat in $x$. The shower coefficient $\alpha_{s h w}(x)$ is relatively small and shows a similar $x$ shape as the energy dependence, which is dictated by the overlapping clusters. The Cherenkov detectors rate dependence 
is relatively large and constant in $x$ except for the lowest $x$ region, where it is smaller. Here the random coincidences start to contribute significantly, and more tracks are gained after spill merger than on average, so the losses due to the dead time are "compensated" by this mechanism. Note that in the lowest $x$ region $\alpha_{\text {total }}$ is higher than the sum $\alpha_{\text {cher }}+\alpha_{\text {hodo }}+\alpha_{\text {shw }}$. This is due to the subtle correlations that exist in tracking. The detectors are sensitive to different types of backgrounds. We thus assume that the rate asymmetries in the individual detector systems are uncorrelated and ignore the mentioned tracking correlations in computing the asymmetry rate dependent correction from eq. (3.17). The rate dependence in the $5.5^{\circ}$ spectrometer was smaller and roughly flat in $x$. The results of the coefficients $\alpha$ averaged over $x$ for all targets and both spectrometer arms are shown in fig. 3.23 Again there is a strong correlation between the rate dependence and the overall rates in spectrometers. The high beam current runs are more rate dependent than the low current runs. The rate dependence was sensitive to the beam quality and spectrometer shielding that were improving in the course of the experiment. There is a jump of the rate in $5.5^{\circ}$ for the target cell Chance. This target had the thickest windows and, as mentioned above, unlike the $2.75^{\circ}$ arm, the $5.5^{\circ}$ spectrometer had fixed acceptance. The rate dependence was typically $6-8 \%$ in the $2.75^{\circ}$ spectrometer and $3-5 \%$ in $5.5^{\circ}$ spectrometer and shows a mild $x$ dependence.

The rate dependence varies with the definition of an electron event. The results of $\alpha_{\text {total }}(x)$ for four different electron choices are shown in fig. 3.24. 

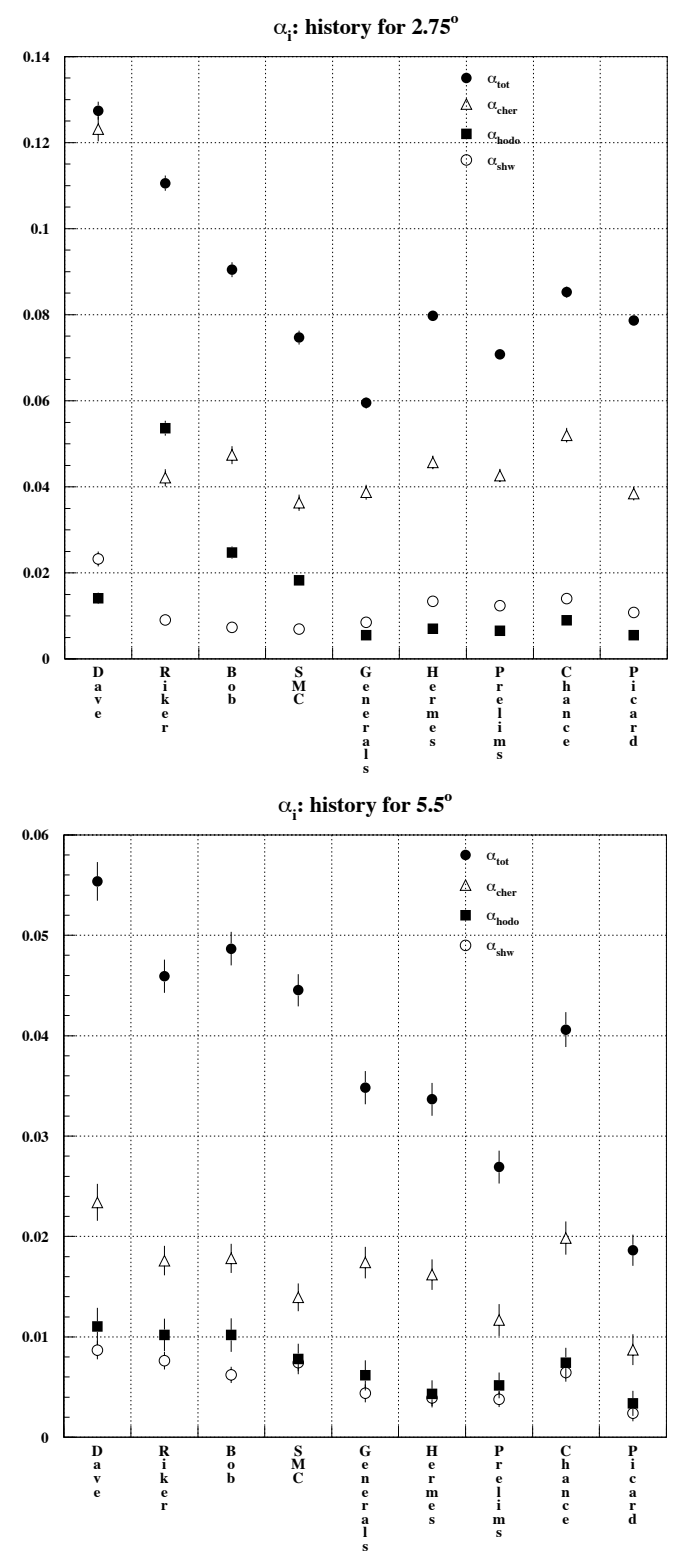

Figure 3.23: Rate dependence coefficients $\alpha$ (see text) for the $2.75^{\circ}$ (top) and $5.5^{\circ}$ (bottom) spectrometers. 


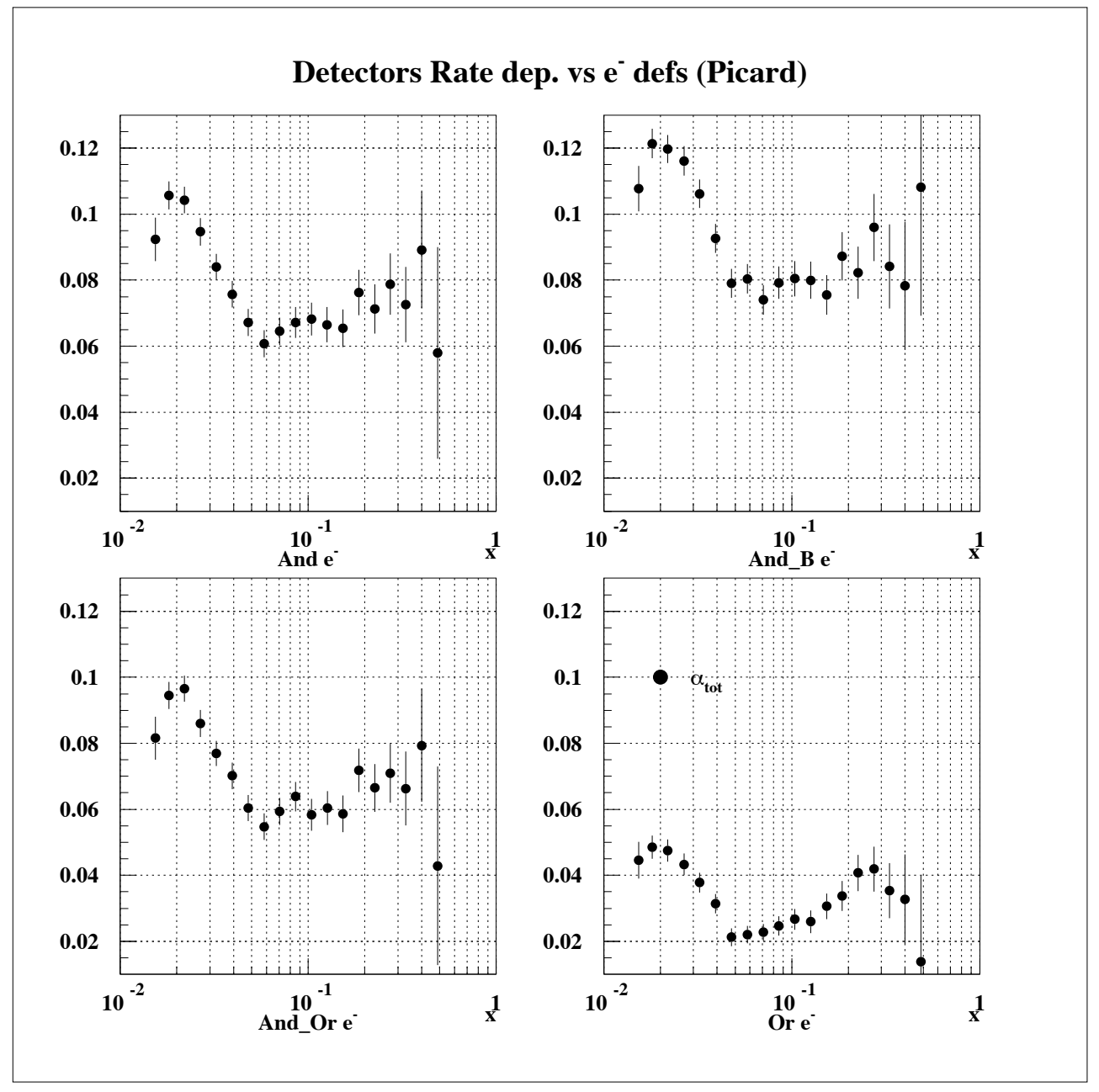

Figure 3.24: Rate dependence of different electron definitions. See text for explanation of And, And_B, And_Or and Or electrons.

The most rate dependent is the "And_B" definition that requires hits in both Cherenkov detectors (meaning of "And" in "And_B") with pulse heights greater than 1.5 photo-electrons and cuts $E / p$ on both ends (meaning of "B" in "And_B"): $0.8 \leq E / p \leq 1.2$. Next is the "And" electron which requires 
Cherenkov hits as above and only $0.8 \leq E / p$. Still less rate dependent is the "And_Or" definition with two Cherenkov hits ("And"), one of them higher than 2.5 photo-electrons ("Or"), and $0.8 \leq E / p$. Finally the least rate sensitive pure "Or" electron with one Cherenkov above 2.5 photo-electrons and $0.8 \leq E / p$. The last definition, however, suffers from high pion background As a compromise the "And_Or" definition was used in the asymmetry analysis.

\subsubsection{Asymmetry correction}

The rate dependence correction is calculated in accordance with eq. (3.17). The detector asymmetries were typically $A^{\text {cher }}=(3-5) \times 10^{-4}$ in the Cherenkov counters, $A^{\text {hodo }}=(1-4) \times 10^{-4}$ in the hodoscopes and $A^{\text {shw }}=$ $(1-4) \times 10^{-3}$ in the calorimeter. The coefficients $\alpha_{i}$ as determined by the pulse fiction method are an upper limit on the true rate dependence. The rate dependence is expected to be a monotonic higher (than linear) order polynomial in the detector rates, see eq. (3.11), with the second power term making the efficiency drop faster. The rate dependence coefficient is proportional to the derivative of the efficiency computed at the single rates $N$. The doubling fusion of pulses approximates this derivative by the slope of the $N$ to $2 N$ line and overestimates its true value. We thus apply only half of the $\Delta A^{\text {rate }}$ correction to the raw asymmetries and use the full value as a systematic error. The error ranges from $4 \%$ of $A_{\|}$at low $x$ to $8 \%$ at high $x$. 


\subsection{Electroweak correction}

Careful consideration of the lepton-nucleon DIS includes scattering with the exchange of the $Z^{\circ}$ boson in addition to virtual photon exchange. Squaring the amplitude results in the interference of the $Z^{\circ}$ and $\gamma$ amplitudes the square of the $Z^{\circ}$ amplitude is negligible) and leads to the parity-violating asymmetry $[71,115]$

$$
A^{E W} \equiv \frac{\sigma_{R}-\sigma_{L}}{\sigma_{R}+\sigma_{L}}=Q^{2}\left(a_{1}+a_{2} \frac{1-(1-y)^{2}}{1+(1-y)^{2}}\right),
$$

where $\sigma_{R}$ and $\sigma_{L}$ are the cross sections for deep-inelastic scattering of a right- and left-handed electrons, and $y=\left(E-E^{\prime}\right) / E$ is the fraction of the energy transfer from the electron to the nucleon. In the ultra-relativistic limit the chirality operator eigenstates coincide with the helicity eigenstates, and thus e.g. the right-handed electrons have positive helicity. In the simplest approximation, when the target is treated as isoscalar and the strange sea is neglected,

$$
\text { and } \quad \begin{aligned}
a_{1} & \approx \frac{3 G_{F}}{5 \sqrt{2} \pi \alpha}\left(-\frac{3}{4}+\frac{5}{3} \sin ^{2} \theta_{W}\right) \\
a_{2} & \approx \frac{9 G_{F}}{5 \sqrt{2} \pi \alpha}\left(\sin ^{2} \theta_{W}-\frac{1}{4}\right),
\end{aligned}
$$

where $G_{F}$ is the Fermi constant, $\alpha$ is the fine structure constant and $\theta_{W}$ is the Weinberg angle. The electroweak asymmetry is not sensitive to the direction of the target polarization and thus is diminished by the target spin reversals. The biggest correction reaches $10 \%$ of $A_{\|}$at high $Q^{2}$ (corresponding to high $x$ ), which is a small fraction of the $A_{\|}$statistical error. We use $20 \%$ of the correction as the systematic uncertainty associated with $A^{E W}$. 


\subsection{Radiative corrections}

The calculation of the polarized structure functions $g_{1}^{n}\left(x, Q^{2}\right)$ and $g_{2}^{n}\left(x, Q^{2}\right)$

and the virtual photon-nucleon asymmetries $A_{1}^{n}\left(x, Q^{2}\right), A_{2}^{n}\left(x, Q^{2}\right)$ from the measured electron cross section asymmetries is based on the single virtual photon exchange diagram. The experimental raw asymmetries, however, include higher order corrections to that diagram as well as the radiative tails from the elastic, quasi-elastic and inelastic regions. In addition the electrons may lose their energy before or after scattering due to the bremsstrahlung or ionization in the external target material. These processes modify the Born asymmetry, so corrections have to be made to extract it from the measured asymmetry.

The radiative effects are traditionally divided into two categories. The "internal" corrections are those occurring at the ${ }^{3}$ He nucleus where the scattering happens. The electrons are off-shell between the emission of the photon and the nuclear scattering. The "external" corrections modify the electron energy before or after the DIS event; the electrons are on-shell.

\subsubsection{Internal radiative corrections}

The kinematics of an event in terms of $x$ and $Q^{2}$ is computed for the single virtual photon exchange process. The higher order processes are included as corrections. They result in the shift of events from higher to lower values of $x$ that are referred to as elastic, quasi-elastic and inelastic tails. The formalism for calculating the radiative corrections ( $\mathrm{RC}$ ) to spin dependent deep 
inelastic scattering has been developed by Kuchto, Shumeiko and Akushevich $[116,117]$ and implemented in their Fortran code POLRAD 1.5 [118]. An independent code based on the ref. [116, 117] was developed at SLAC by Linda Stuart [119] and produced identical results.

The measured DIS unpolarized cross section $\sigma^{\mathrm{m}}\left(x, Q^{2}\right)$ can be written in terms of the Born $\sigma^{\mathrm{B}}\left(x, Q^{2}\right)$ and tails cross section $\sigma^{\text {tails }}\left(x, Q^{2}\right)$ as

$$
\sigma^{\mathrm{m}}=\sigma^{\mathrm{B}} V+\sigma^{\mathrm{tails}}
$$

where $V$ is the correction due to higher orders, given by

$$
V=\delta_{R}^{I R}+\delta_{\mathrm{vert}}+\delta_{\mathrm{vac}}^{l}+\delta_{\mathrm{vac}}^{h}
$$

Here $\delta_{R}^{I R}$ is due to the soft photon emission, $\delta_{\text {vert }}$ is the lepton vertex correction, $\delta_{\text {vac }}^{l}$ refers to the lepton vacuum polarization and $\delta_{\text {vac }}^{h}$ is the hadronic vacuum polarization. The corrections are insensitive to the spin interaction and thus are the same for the polarized cross section,

$$
\Delta \sigma^{\mathrm{m}}=\Delta \sigma^{\mathrm{B}} V+\Delta \sigma^{\mathrm{tails}}
$$

The measured asymmetry $A^{\mathrm{m}}=\sigma^{\mathrm{m}} / \Delta \sigma^{\mathrm{m}}$ is related to the Born asymmetry $A^{\mathrm{B}}=\sigma^{\mathrm{B}} / \Delta \sigma^{\mathrm{B}}$ via eq. $(3.20),(3.22)$,

$$
A^{\mathrm{m}}=A^{\mathrm{B}} \frac{\sigma^{\mathrm{B}} V}{\sigma^{\mathrm{B}} V+\sigma^{\text {tails }}}+\frac{\Delta \sigma^{\text {tails }}}{\sigma^{\mathrm{B}} V+\sigma^{\text {tails }}} .
$$

The fraction

$$
f \equiv \frac{\sigma^{\mathrm{B}} V}{\sigma^{\mathrm{B}} V+\sigma^{\text {tails }}}
$$


is the dilution due to the tails, while the ratio

$$
\Delta A^{\text {tails }} \equiv \frac{\Delta \sigma^{\text {tails }}}{\sigma^{\mathrm{B}} V}
$$

is the tail asymmetry, so that

$$
A^{\mathrm{B}}=\frac{A^{\mathrm{m}}}{f}-\Delta A^{\mathrm{tails}} .
$$

Traditionally the radiative correction is defined additively,

$$
\Delta A^{\mathrm{RC}} \equiv A^{\mathrm{B}}-A^{\mathrm{m}}
$$

Although this is a convenient way of characterizing the size of the correction, we have to refer to eq. (3.26) for the proper propagation of uncertainties, or to remember that $\Delta A^{\mathrm{RC}}$ is correlated with the measured $A^{\mathrm{m}}$.

The tail cross section has contributions from the elastic, quasi-elastic, resonance and DIS tails,

$$
\sigma^{\mathrm{tails}}=\sigma^{\mathrm{el}}+\sigma^{\mathrm{qel}}+\sigma^{\mathrm{res}}+\int_{x^{\prime}>x} d x^{\prime} \int d Q^{\prime 2} \sigma^{\mathrm{DIS}}\left(x^{\prime}, Q^{\prime 2}\right) .
$$

The elastic cross section arises from elastic electron scattering off the whole ${ }^{3} \mathrm{He}$ nucleus and is characterized by the ${ }^{3}$ He form-factor. The whole contribution to the lowest $x \approx 0.14$ from unpolarized and polarized tails comes from a very low $Q^{2} \sim 10^{-3} \mathrm{GeV}^{2}$ [83]. It corresponds to the electron emitting a hard photon at a large angle that deflects by about $2.75^{\circ}$ and then scatters elastically through a small angle (or first scatters elastically through a small angle and then emits a photon).

The quasi-elastic tail comes from the elastic scattering of the neutron or proton inside the ${ }^{3}$ He nucleus. The emission of a hard photon by the 
incident electron before scattering lowers the energy and $Q^{2}$ of the event. Since the form factors of the nucleon are proportional to $1 / Q^{4}$ at high $Q^{2}$, the probability of scattering quasi-elastically is enhanced. The asymmetry of the quasi-elastic scattering, expressible in terms of products of form factors $G_{E} G_{M}$ and $G_{M}^{2}$ [120], is mixed with the DIS asymmetry. Details of the nuclear structure of ${ }^{3} \mathrm{He}$ are important in the evaluation of this contribution. Predictions for the S, S' and D percentages of the ${ }^{3}$ He wave function (see sec. 1.4, page 12) are used to determine the relative contributions from the quasi-elastic asymmetries of the protons and neutrons inside the ${ }^{3}$ He nuclei. The contribution to the lowest $x$ from these polarized and unpolarized tails comes predominantly from $Q^{2}=0.23 \mathrm{GeV}^{2}$ [83]. It corresponds to electrons losing most of their energy by emitting a photon before scattering: $E=E^{\prime}=$ $10 \mathrm{GeV}, \theta=2.75^{\circ} \Rightarrow Q^{2}=4 E E^{\prime} \sin ^{2}(\theta / 2)=0.23 \mathrm{GeV}^{2}$.

The inelastic tail contribution arises similarly to the processes discussed above. The incident energy $E$ of electrons detected in the spectrometers that undergo hard photon emission before scattering is overestimated. The event is assigned a higher value of $Q^{2}\left(Q^{2}=4 E E^{\prime} \sin ^{2}(\theta / 2)\right)$ and lower value of $x$ $\left(x=Q^{2} / 2 M\left(E-E^{\prime}\right)\right)$. Similarly, bremsstrahlung after the scattering results in underestimation of the scattered electron energy E'. The event is assigned a lower value of $Q^{2}$ and a lower value of $x$. Therefore, DIS asymmetries from higher $x$ as well as asymmetries from the resonance region $W^{2}<4 \mathrm{GeV}^{2}$ are mixed into the asymmetry measured at lower $x$. Once again the large contribution from the resonance region to the lowest $x$ inelastic tail comes 
from $Q^{2} \sim 0.25 \mathrm{GeV}^{2}$, which corresponds to electrons losing most of their energy by emitting a photon before scattering.

The various tail contributions to the unpolarized cross section as a function of $x$ for the $2.75^{\circ}$ spectrometer are shown in fig. 3.25 . The corrections

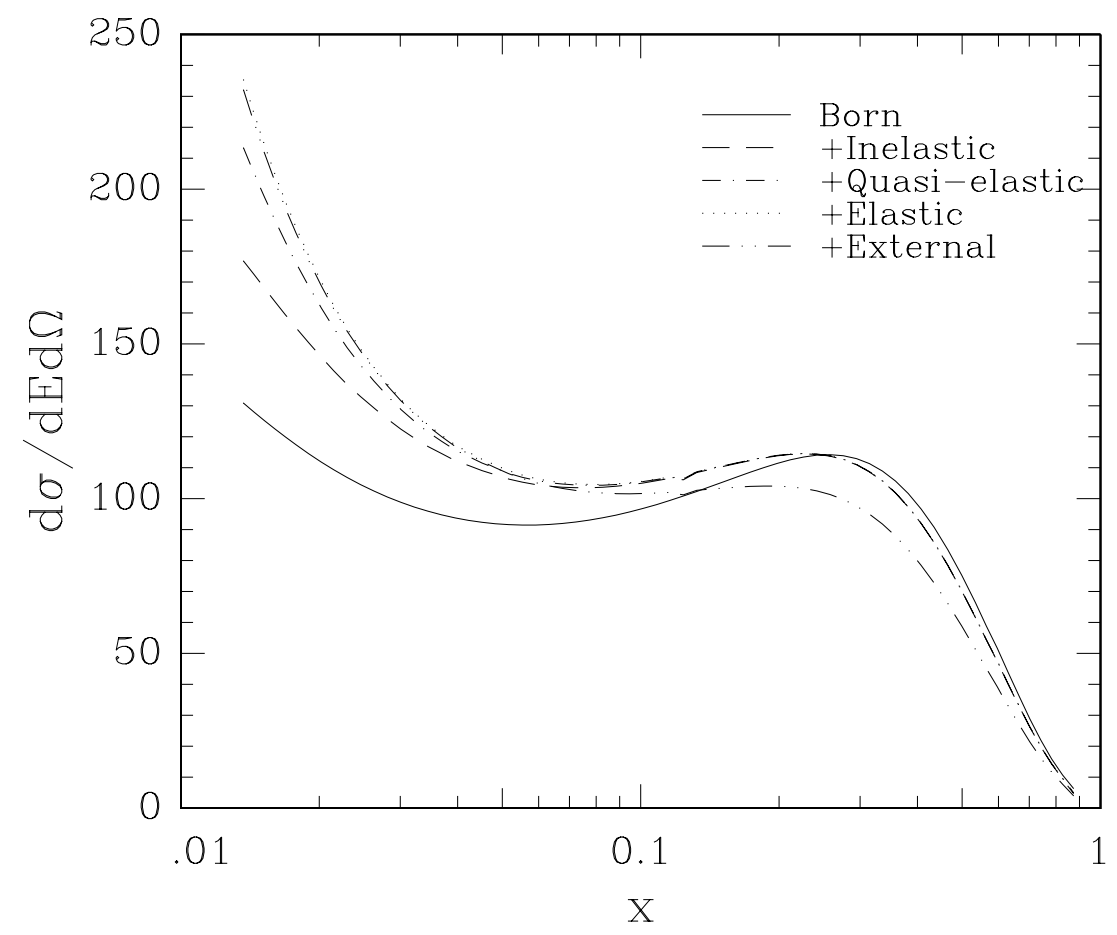

Figure 3.25: The unpolarized Born cross section and various radiative tails in the $2.75^{\circ}$ spectrometer.

are dominated by the inelastic and quasi-elastic tail at low $x$. At high $x$ the external corrections become significant. The polarized tails are shown in fig. 3.26. The polarized Born cross section is ten times larger than the largest tail. The elastic tail makes a large contribution at low $x$ because it has a large asymmetry. 


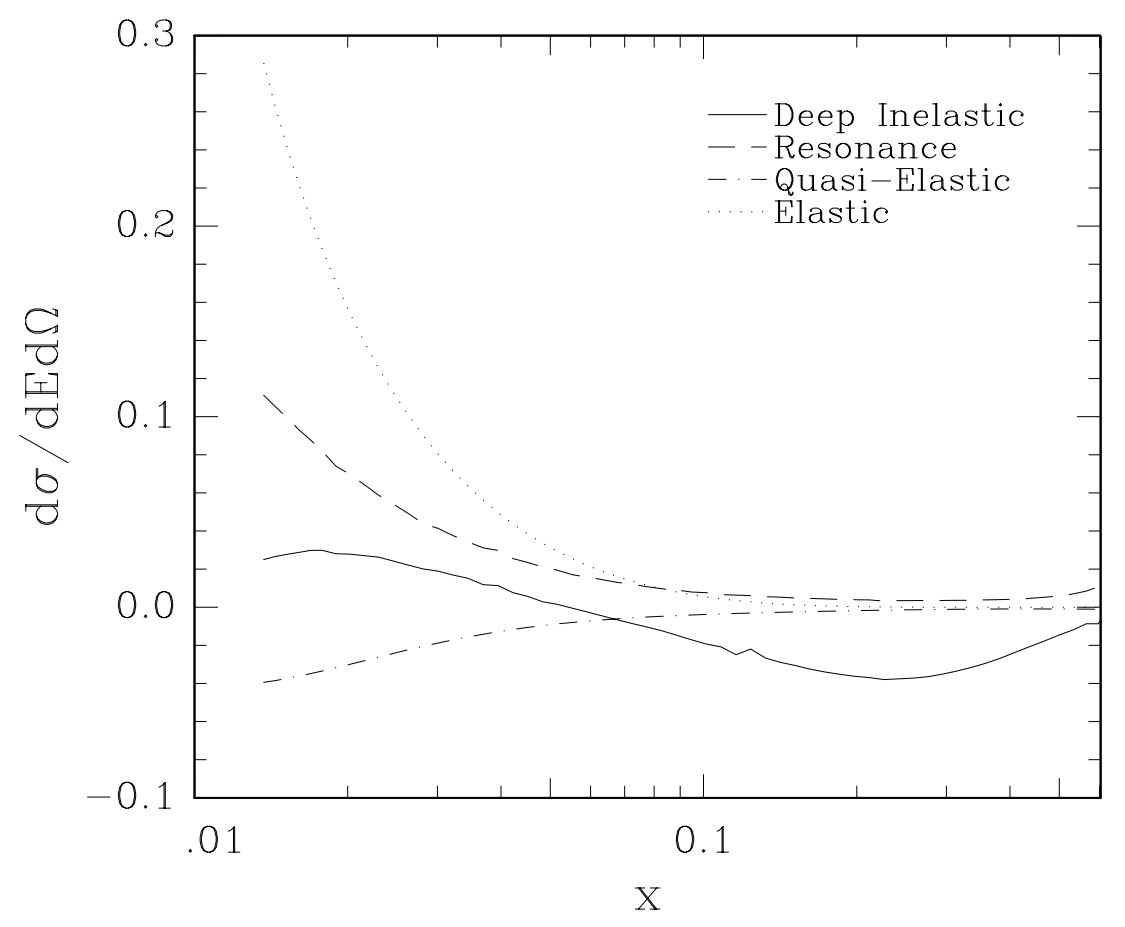

Figure 3.26: The polarized radiative tails in the $2.75^{\circ}$ spectrometer.

\subsubsection{External radiative corrections}

The electrons lose their energy before or after scattering by ionization and, to a much greater extent, by bremsstrahlung interactions with other target material. These "external" effects make the measured uncorrected asymmetry a convolution of polarized and unpolarized cross sections with electron straggling probabilities over a broad kinematic region of $x$ greater than the measured $x$. The formalism of Mo and Tsai [121, 122] was used to compute the external radiative corrections. The dominant source of external radiative corrections is the side walls of the target cell traversed by the elec- 
tron at very shallow angles. Overall, the effect of those corrections on the measured asymmetry is small.

\subsubsection{Radiative corrections results}

The uncertainty of the extracted $A^{\mathrm{B}}$ asymmetry has three sources: the statistical and systematic (excluding $\mathrm{RC}$ ) error of the measured $A^{\mathrm{m}}$ asymmetry and the genuine RC systematic error. The statistical errors are uncorrelated, and thus their contributions to a given $x$ bin $i$ are added in quadrature (see eq. $(3.26))$ :

$$
\sigma_{\text {stat }}^{2}\left(A_{i}^{\mathrm{B}}\right)=\sigma_{\text {stat }}^{2}\left(A_{i}^{\mathrm{m}}\right)\left(\frac{1}{f}\right)^{2}+\sum_{j \neq i} \sigma_{\text {stat }}^{2}\left(A_{j}^{\mathrm{m}}\right)\left(\frac{\partial \Delta A^{\mathrm{tails}}}{\partial A_{j}^{\mathrm{m}}}\right)^{2} .
$$

The systematic errors are assumed to be $100 \%$ correlated from point to point, and their contributions in a given $x$ bin $i$ are added linearly:

$$
\sigma_{\mathrm{syst}}\left(A_{i}^{\mathrm{B}}\right)=\sigma_{\mathrm{syst}}\left(A_{i}^{\mathrm{m}}\right)\left(\frac{1}{f}\right)+\sum_{j \neq i} \sigma_{\mathrm{syst}}\left(A_{j}^{\mathrm{m}}\right)\left(\frac{\partial \Delta A^{\mathrm{tails}}}{\partial A_{j}^{\mathrm{m}}}\right) .
$$

The radiative dilution $f$ can be written as (cf. eq. (3.20), (3.24))

$$
f=\frac{\sigma^{\mathrm{m}}-\sigma^{\mathrm{tails}}}{\sigma^{\mathrm{m}}}
$$

Due to the infra-red divergence [123] the definition of the DIS tail is ambiguous (the ambiguity cancels out with the ambiguity coming from the virtual corrections, so that $\sigma^{\mathrm{m}}$ is unambiguous). We include in $\sigma^{\text {DIS }}$ those points that are more than two bins away from the $x$ of the measurement.

The systematic uncertainty of the radiative corrections was estimated by varying the input models of the unpolarized and polarized cross sections: the 
form factors for elastic and quasi-elastic scatterings, nuclear corrections in ${ }^{3} \mathrm{He}$ unpolarized structure functions, models of the resonance region, contributions from $g_{2}$ and possible $Q^{2}$ dependence [60] of the ratio $g_{1} / F_{1}$ below $Q^{2}$ of $1 \mathrm{GeV}^{2}$; and the target model (for the external corrections). The results for the radiative corrections to $A_{\|}$and their uncertainties are given in table 3.2 .

Table 3.2: Radiative Corrections $(\times 100)$ and Their Systematic Errors $(\times$ 100).

\begin{tabular}{|c|c|c|c|}
\hline$x$ bin & $\Delta A^{\mathrm{RC}}$ & $\sigma\left(A^{\mathrm{B}}\right) / \sigma\left(A^{\mathrm{m}}\right)$ & Syst. \\
\hline \multicolumn{4}{|c|}{$2.75^{\circ}$ spectrometer } \\
\hline 0.017 & -0.341 & 1.686 & 0.051 \\
\hline 0.025 & -0.285 & 1.500 & 0.057 \\
\hline 0.035 & -0.233 & 1.334 & 0.038 \\
\hline 0.049 & -0.192 & 1.216 & 0.022 \\
\hline 0.078 & -0.151 & 1.154 & 0.017 \\
\hline 0.123 & -0.122 & 1.113 & 0.019 \\
\hline 0.173 & -0.099 & 1.068 & 0.015 \\
\hline 0.241 & -0.081 & 1.049 & 0.018 \\
\hline 0.340 & -0.061 & 1.048 & 0.026 \\
\hline 0.423 & -0.051 & 1.102 & 0.046 \\
\hline \multicolumn{4}{|c|}{$5.5^{\circ}$ spectrometer } \\
\hline 0.0573 & -0.290 & 1.319 & 0.070 \\
\hline 0.0837 & -0.251 & 1.202 & 0.053 \\
\hline 0.1231 & -0.227 & 1.123 & 0.037 \\
\hline 0.1725 & -0.210 & 1.066 & 0.035 \\
\hline 0.2420 & -0.185 & 1.039 & 0.022 \\
\hline 0.3424 & -0.152 & 1.022 & 0.020 \\
\hline 0.4423 & -0.124 & 1.009 & 0.030 \\
\hline 0.5643 & -0.102 & 1.028 & 0.063 \\
\hline
\end{tabular}




\subsection{Dilution factor}

The physics asymmetry is diluted by the events initiated at the unpolarized material of the target. We need to estimate the dilution factor $f$ defined as

$$
f=\frac{\text { number of } \mathrm{e}^{-} \text {scattered off }{ }^{3} \mathrm{He}}{\text { number of } \mathrm{e}^{-} \text {scattered off the target as a whole }} .
$$

About half of the events came from the ${ }^{3} \mathrm{He}$, while the rest from the glass target windows and (a small fraction) from the nitrogen. The dilution factor was computed from the target model and the known unpolarized cross sections. It was also measured from the rates of the reference cell filled with a variable amount of ${ }^{3} \mathrm{He}$ in the spectrometers (see appendix A, page 246 for a detailed description).

\subsubsection{Model calculation}

The composition and dimensions of the target [87] allow us to predict the dilution factor,

$$
f\left(x, Q^{2}\right)=\frac{R_{H e}\left(x, Q^{2}\right)}{R_{H e}\left(x, Q^{2}\right)+R_{N}\left(x, Q^{2}\right)+R_{g l}\left(x, Q^{2}\right)},
$$

where $R_{H e, N, g l}$ denotes the rates from helium, nitrogen and target cell glass. The rates from an element $i$ are given by the formula

$$
R_{i}=\left(Z_{i} F_{2}^{p}+\left(A_{i}-Z_{i}\right) F_{2}^{n}\right) f_{E M C}^{i} f_{R C}^{i} L_{i} n_{i}
$$

Here $A_{i}$ is the atomic number, and $Z_{i}$ is the number of protons of an element

$i, L_{i}$ is the thickness of the material and $n_{i}$ its number density, $f_{R C}^{i}$ is the 
multiplicative unpolarized radiative correction (cf. section 3.9 ), $f_{E M C}^{i}$ is the EMC factor [29] and $F_{2}^{p}, F_{2}^{n}$ are the unpolarized structure functions [124] for the proton and neutron.

On average $56 \%$ of our events come from scattering of the ${ }^{3} \mathrm{He}, 40 \%$ from glass and $4 \%$ from the nitrogen. The biggest systematic uncertainty is due to the radiative corrections. It ranges from $5 \%$ at low $x$ and $4 \%$ at high $x$ to $1 \%$ at $\operatorname{mid} x$. The errors in $F_{2}$ and the window thickness contribute about $1 \%$.

\subsubsection{Measurement}

Special runs were taken during the experiment with the reference cell filled with a variable pressure of ${ }^{3} \mathrm{He}$. They permitted the measurement of the dilution factor. The geometry of the reference cell was the same as the target cell. We measure the rates $R_{\text {empty }}$ from the empty reference cell and the rates $R_{\text {full }}$ from the reference cell with the same amount of ${ }^{3}$ He as in the target cell. Then

$$
f\left(x, Q^{2}\right)=\frac{R_{\mathrm{full}}\left(x, Q^{2}\right)-R_{\mathrm{empty}}\left(x, Q^{2}\right)}{R_{\mathrm{tg}}\left(x, Q^{2}\right)},
$$

where $R_{\mathrm{tg}}$ are the rates from the target cell. The rates $R_{\text {ref }}$ in the reference cell vary linearly with the ${ }^{3} \mathrm{He}$ pressure $P_{\mathrm{He}}$. Thus the rates from ${ }^{3} \mathrm{He}$ in the target cell can be obtained from the slope $\partial R_{\text {ref }} / \partial P_{\mathrm{He}}$ and the pressure $P_{\mathrm{He}}^{\mathrm{tg}}$ of the ${ }^{3}$ He inside the target cell. In practice the difference in the target and reference cell lengths has to be taken into account, so

$$
f=\frac{\partial R_{\mathrm{ref}}}{\partial P_{\mathrm{He}}} \frac{P_{\mathrm{He}}^{\mathrm{tg}}}{R_{\mathrm{tg}}} \frac{L_{\mathrm{tg}}}{L_{\mathrm{ref}}},
$$


where $L_{\mathrm{tg}}, L_{\mathrm{ref}}$ are the lengths of the target and reference cell respectively. The rates were corrected for the backgrounds and rate dependence. The uncertainty of the resulted dilution factor was mainly due to the statistics of the measurement.

\subsubsection{Comparison and results}

The theoretical and experimental dilution factors are compared in fig. 3.27. The agreement is very good. For the asymmetry analysis we use the model
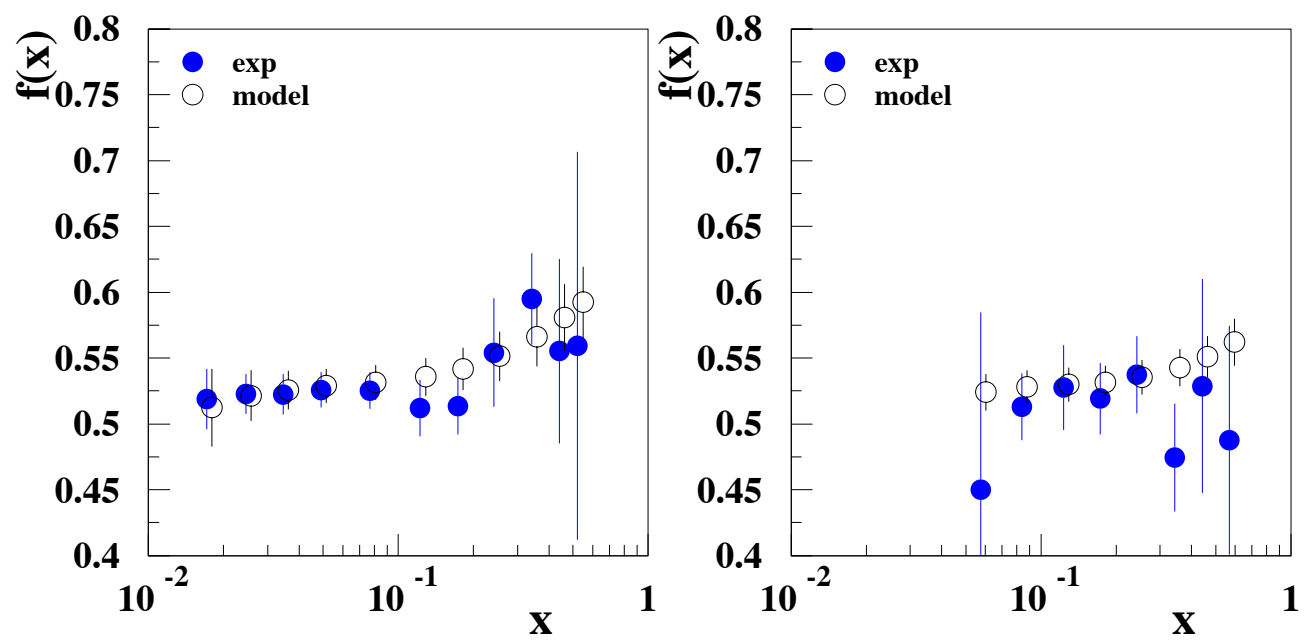

Figure 3.27: Comparison of the theoretical (open circles) with experimental (closed circles) dilution factors for $2.75^{\circ}$ (left) and $5.5^{\circ}$ (right) spectrometers, target cell Picard.

result. The uncertainty comes from the systematic model error added in quadrature with the disagreement between the two methods. The average uncertainty of the dilution factor, weighted by the statistical error of $A_{\|}$, is $5.1 \%$. 


\subsection{Asymmetry results for ${ }^{3} \mathrm{He}$}

The measured asymmetries are corrected for the backgrounds, rate dependence, electroweak $Z^{\circ}$ exchange interference, polarization dilutions and radiative corrections as given by eq. (3.8), page 123 :

$$
A^{\text {phys }}=\frac{A_{e}^{\text {raw }}+\Delta A^{\text {rate }}-P_{b} A^{E W}}{P_{b} P_{t} f}+\Delta A^{R C},
$$

where $A_{e}^{\text {raw }}$ is the subtracted background in accordance with eq. 3.10, page 124,

$$
A_{e}^{\text {raw }}=\frac{1}{1-e^{+} / e}\left[\frac{A^{\text {raw }}-(\pi / e) A_{\pi}}{1-\pi / e}-\left(e^{+} / e\right) A_{e^{+}}\right] \text {. }
$$

The physics asymmetries $A^{\text {phys }}$ are computed for every run and averaged with the weights given by statistical error. Simultaneously the average values of $\langle x\rangle$ and $\left\langle Q^{2}\right\rangle$ are calculated. The results for the $A_{\|}$and $A_{\perp}$ of ${ }^{3}$ He are given in table 3.3 [91].

\subsection{Determination of the neutron structure functions}

The two independent analyses performed at SLAC and Caltech were compared for $A_{\|}$and $A_{\perp}$ asymmetries of ${ }^{3}$ He and were found to be in very good agreement. The differences were consistent with the statistical fluctuations due to the $10-15 \%$ difference in the event samples. For the publications the Collaboration decided to simply (no weights) average the two results and to adopt the larger statistical error. The averaged asymmetries are given in table 3.4 . 


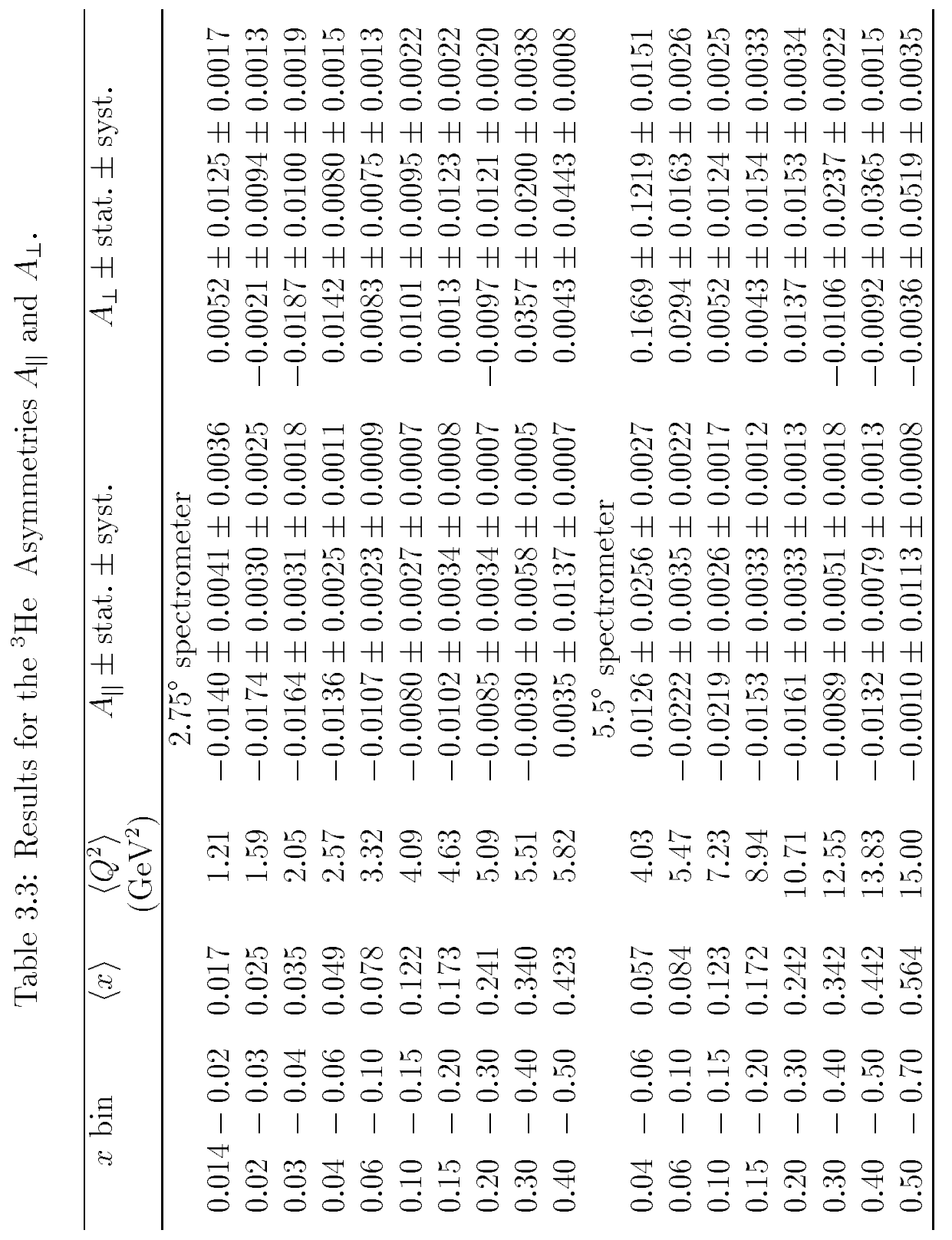




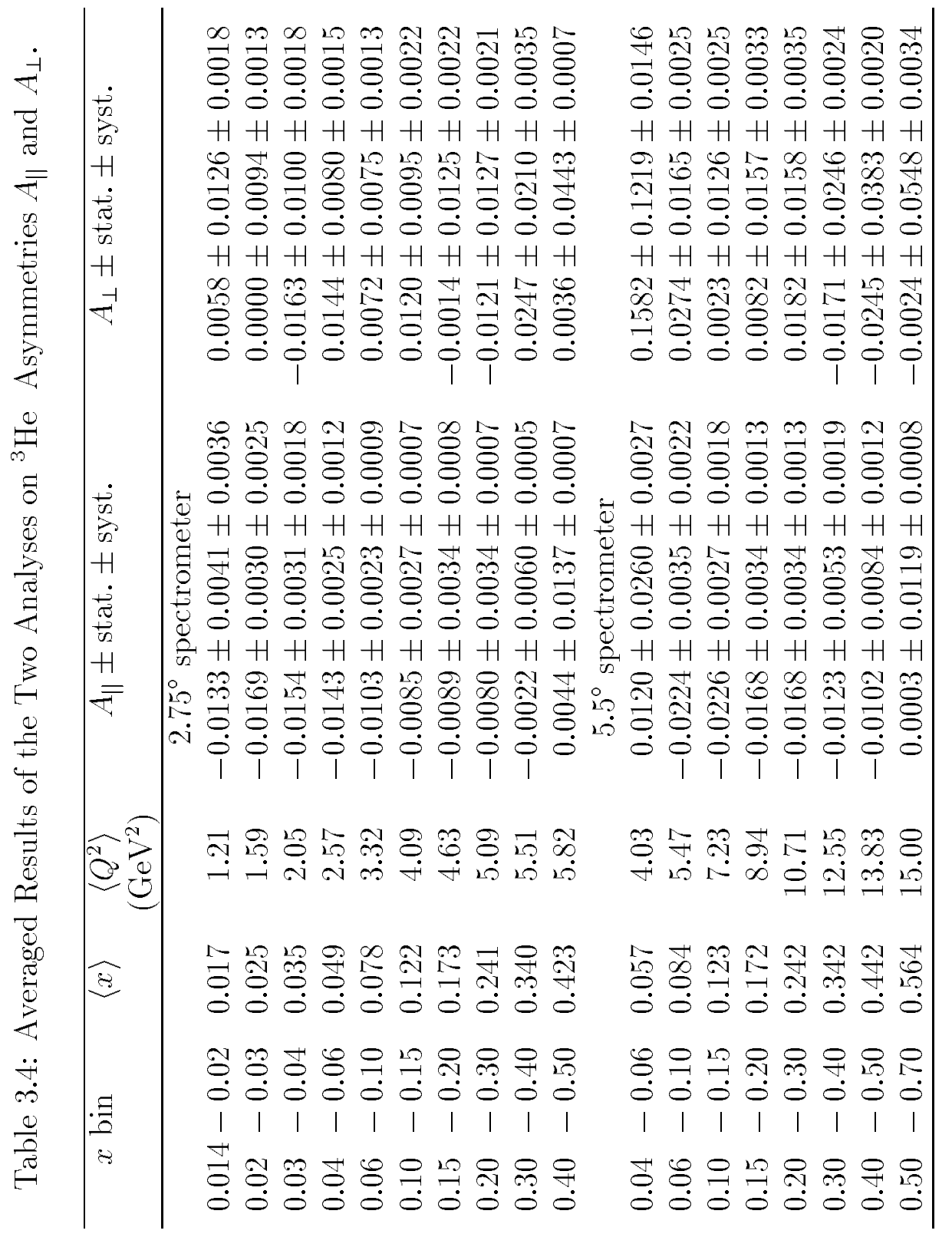


The averaged ${ }^{3}$ He asymmetries $A_{\|}^{3} H e$ and $A_{\perp}^{3} H e$ are used to calculate $A_{\|}^{n}$ and $A_{\perp}^{n}$ of the neutron (see eq. (1.46), page 13 ),

$$
A_{\|}^{n}=\frac{1}{p_{n} F_{2}^{n}}\left(A_{\|}^{3} H e\left(2 F_{2}^{p}+F_{2}^{n}\right) f_{E M C}-2 A_{\|}^{p} p_{p} F_{2}^{p}\right) \text {, }
$$

and similarly for $A_{\perp}^{n}$. Here $p_{n(p)}$ is the neutron (proton) polarization in ${ }^{3} \mathrm{He}$ (see sec. 1.4, page 12), $f_{E M C}$ is the the EMC effect factor [29], and $F_{2}^{n(p)}$ is the unpolarized structure function of the neutron (proton). We assume that the ratio $R\left(x, Q^{2}\right)$ of the longitudinal to transverse virtual photon-nucleon cross sections is the same for the proton and neutron $[24,25]$. We use the fit to the world data on the $g_{1}^{p}$ structure function $[18,20]$ to calculate the proton asymmetry $A_{\|}^{p}$. From the fit we also calculate the $g_{2}^{p}$ contribution in terms of the Wandzura-Wilczek twist-2 expression (see eq. (1.129), page 30).

The spin dependent structure functions $g_{1}^{n}$ and $g_{2}^{n}$ of the neutron were calculated from eq. (1.17), (1.18), page 8, and the virtual photon-neutron asymmetries $A_{1}^{n}$ and $A_{2}^{n}$ from eq. (1.34), (1.35), page 11. The results are given in tables 3.5 and 3.6 for both spectrometers. The product $x g_{1}^{n}$ is plotted in fig. 3.28 and $x g_{2}^{n}$ in fig. 3.29. The results for $g_{1}^{n}$ and $A_{1}^{n}$ from both spectrometers were averaged, weighted by the statistical errors. The average $\langle x\rangle$ and $\left\langle Q^{2}\right\rangle$ were weighted by the statistical errors of $g_{1}^{n}$. The values are given in table 3.7. For the evaluation of the QCD sum rules, defined at a fixed four-momentum transfer, the data points for the structure function $g_{1}^{n}$ are evolved to a common value of $Q^{2}$. Usually the average $\left\langle Q^{2}\right\rangle$ of the experiment is taken as the fixed value, so the evolution in $Q^{2}$ is minimal (on average). The average $\left\langle Q^{2}\right\rangle$, weighted by statistics of $A_{\|}$, is $5 \mathrm{GeV}^{2}$ for the E154 experiment. 
Table 3.5: The Spin Dependent Structure Function $g_{1}^{n}$ and the Virtual Photon-Nucleon Asymmetry $A_{1}^{n}$.

\begin{tabular}{ccrr}
\hline$\langle x\rangle$ & $\left\langle Q^{2}\right\rangle\left(\mathrm{GeV}^{2}\right)$ & \multicolumn{1}{c}{$g_{1}^{n} \pm$ stat. \pm syst. } & \multicolumn{1}{c}{$A_{1}^{n} \pm$ stat. \pm syst. } \\
\hline & & $2.75^{\circ}$ spectrometer & \\
0.017 & 1.21 & $-0.351 \pm 0.115 \pm 0.109$ & $-0.058 \pm 0.019 \pm 0.017$ \\
0.024 & 1.59 & $-0.374 \pm 0.071 \pm 0.064$ & $-0.080 \pm 0.015 \pm 0.014$ \\
0.035 & 2.05 & $-0.289 \pm 0.061 \pm 0.038$ & $-0.078 \pm 0.018 \pm 0.011$ \\
0.049 & 2.57 & $-0.212 \pm 0.041 \pm 0.022$ & $-0.089 \pm 0.016 \pm 0.010$ \\
0.078 & 3.32 & $-0.119 \pm 0.031 \pm 0.013$ & $-0.078 \pm 0.019 \pm 0.009$ \\
0.123 & 4.09 & $-0.075 \pm 0.030 \pm 0.010$ & $-0.089 \pm 0.031 \pm 0.011$ \\
0.173 & 4.63 & $-0.070 \pm 0.033 \pm 0.010$ & $-0.100 \pm 0.053 \pm 0.014$ \\
0.241 & 5.09 & $-0.053 \pm 0.028 \pm 0.008$ & $-0.078 \pm 0.077 \pm 0.018$ \\
0.340 & 5.51 & $0.002 \pm 0.036 \pm 0.004$ & $-0.166 \pm 0.206 \pm 0.051$ \\
0.423 & 5.82 & $0.027 \pm 0.059 \pm 0.007$ & $0.166 \pm 0.606 \pm 0.038$ \\
& & $5.5^{\circ}$ spectrometer & \\
0.057 & 4.03 & $0.224 \pm 0.285 \pm 0.035$ & $0.045 \pm 0.120 \pm 0.012$ \\
0.084 & 5.47 & $-0.152 \pm 0.029 \pm 0.019$ & $-0.104 \pm 0.018 \pm 0.013$ \\
0.123 & 7.23 & $-0.117 \pm 0.017 \pm 0.013$ & $-0.110 \pm 0.015 \pm 0.012$ \\
0.172 & 8.94 & $-0.059 \pm 0.016 \pm 0.009$ & $-0.090 \pm 0.023 \pm 0.011$ \\
0.242 & 10.71 & $-0.040 \pm 0.012 \pm 0.006$ & $-0.118 \pm 0.030 \pm 0.016$ \\
0.342 & 12.55 & $-0.019 \pm 0.012 \pm 0.005$ & $-0.057 \pm 0.068 \pm 0.022$ \\
0.442 & 13.83 & $-0.009 \pm 0.012 \pm 0.003$ & $-0.013 \pm 0.146 \pm 0.018$ \\
0.564 & 15.00 & $0.003 \pm 0.008 \pm 0.001$ & $0.100 \pm 0.294 \pm 0.032$ \\
\hline
\end{tabular}

The evolution is traditionally based on the assumption that the ratio of the polarized to the unpolarized structure functions, $g_{1} / F_{1}$, or asymmetry $A_{1}$ scales in $Q^{2}$ i.e. is $Q^{2}$ independent for all $x$. Although these assumptions contradict the pQCD, they are compatible with the experimental evidence and could be a reasonable approximation for a short range of the $Q^{2}$ evolution and/or if the error of the approximation is small in comparison with the precision of the measurements. The next-to-leading order pQCD evolution 
Table 3.6: The Spin Dependent Structure Function $g_{2}^{n}$ and the Virtual Photon-Nucleon Asymmetry $A_{2}^{n}$.

\begin{tabular}{ccrr}
\hline$\langle x\rangle$ & $\left\langle Q^{2}\right\rangle\left(\mathrm{GeV}^{2}\right)$ & \multicolumn{1}{c}{$g_{2}^{n} \pm$ stat. \pm syst. } & \multicolumn{1}{c}{$A_{2}^{n} \pm$ stat. \pm syst. } \\
\hline & \multicolumn{3}{c}{$2.75^{\circ}$ spectrometer } \\
0.017 & 1.21 & $7.167 \pm 15.312 \pm 2.169$ & $0.033 \pm 0.074 \pm 0.010$ \\
0.024 & 1.59 & $0.154 \pm 7.232 \pm 0.980$ & $-0.002 \pm 0.056 \pm 0.007$ \\
0.035 & 2.05 & $-7.870 \pm 4.890 \pm 0.958$ & $-0.106 \pm 0.064 \pm 0.013$ \\
0.049 & 2.57 & $4.605 \pm 2.504 \pm 0.543$ & $0.099 \pm 0.056 \pm 0.012$ \\
0.078 & 3.32 & $1.318 \pm 1.331 \pm 0.245$ & $0.058 \pm 0.065 \pm 0.012$ \\
0.123 & 4.09 & $1.223 \pm 0.953 \pm 0.237$ & $0.127 \pm 0.106 \pm 0.026$ \\
0.173 & 4.63 & $-0.080 \pm 0.810 \pm 0.145$ & $-0.033 \pm 0.179 \pm 0.033$ \\
0.241 & 5.09 & $-0.486 \pm 0.515 \pm 0.105$ & $-0.251 \pm 0.241 \pm 0.049$ \\
0.340 & 5.51 & $0.541 \pm 0.466 \pm 0.145$ & $0.635 \pm 0.550 \pm 0.126$ \\
0.423 & 5.82 & $0.040 \pm 0.580 \pm 0.018$ & $0.162 \pm 1.414 \pm 0.040$ \\
& & $5.5^{\circ}$ spectrometer & \\
0.057 & 4.03 & $41.007 \pm 31.640 \pm 4.458$ & $0.945 \pm 0.727 \pm 0.103$ \\
0.084 & 5.47 & $4.077 \pm 2.403 \pm 0.434$ & $0.161 \pm 0.099 \pm 0.018$ \\
0.123 & 7.23 & $0.231 \pm 1.003 \pm 0.196$ & $0.009 \pm 0.080 \pm 0.016$ \\
0.172 & 8.94 & $0.398 \pm 0.723 \pm 0.153$ & $0.052 \pm 0.112 \pm 0.024$ \\
0.242 & 10.71 & $0.477 \pm 0.407 \pm 0.098$ & $0.145 \pm 0.136 \pm 0.031$ \\
0.342 & 12.55 & $-0.216 \pm 0.311 \pm 0.039$ & $-0.205 \pm 0.273 \pm 0.032$ \\
0.442 & 13.83 & $-0.155 \pm 0.239 \pm 0.030$ & $-0.360 \pm 0.532 \pm 0.047$ \\
0.564 & 15.00 & $-0.008 \pm 0.132 \pm 0.009$ & $-0.036 \pm 0.953 \pm 0.058$ \\
\hline
\end{tabular}

will be applied in chapter 4 (page 176 ). At the moment we adopt the $g_{1} / F_{1}$ scaling assumption and evolve the results for $g_{1}^{n}$ from both spectrometers separately to $5 \mathrm{GeV}^{2}$ and then average them with the weights given by the evolved statistical error. The results are quoted in table 3.7 and plotted in fig. 3.30 .

The systematic uncertainties in the $g_{1}^{n}$ structure function are summarized in table 3.8 . The contributions to each $x$ point and to the integral over 


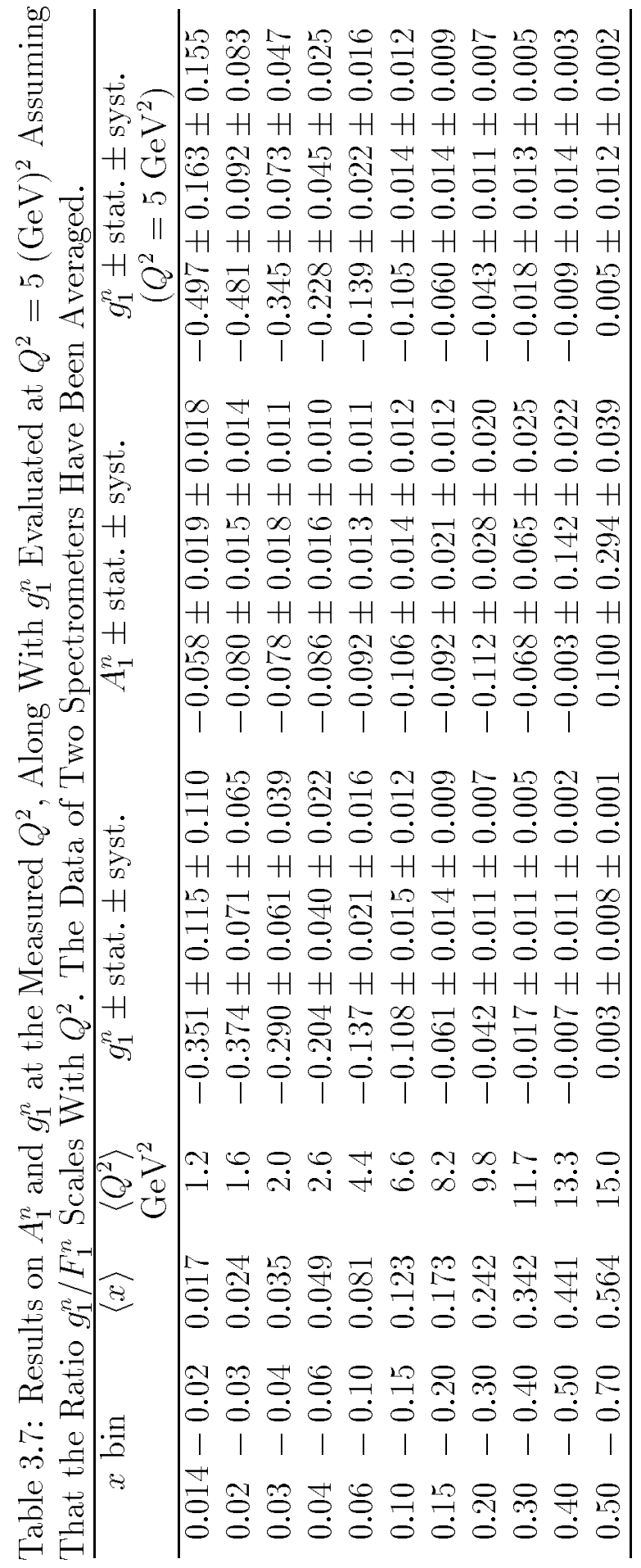




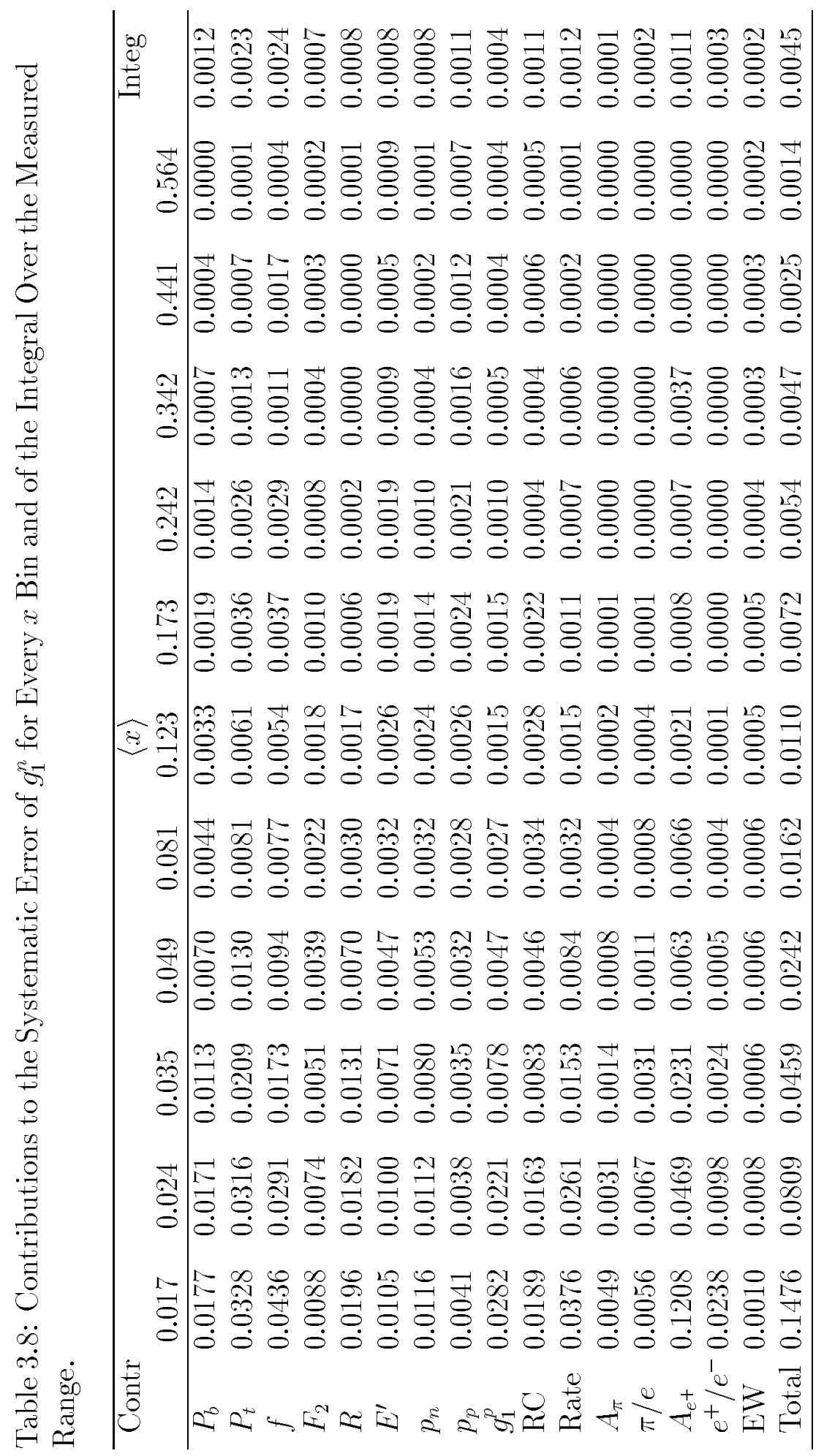




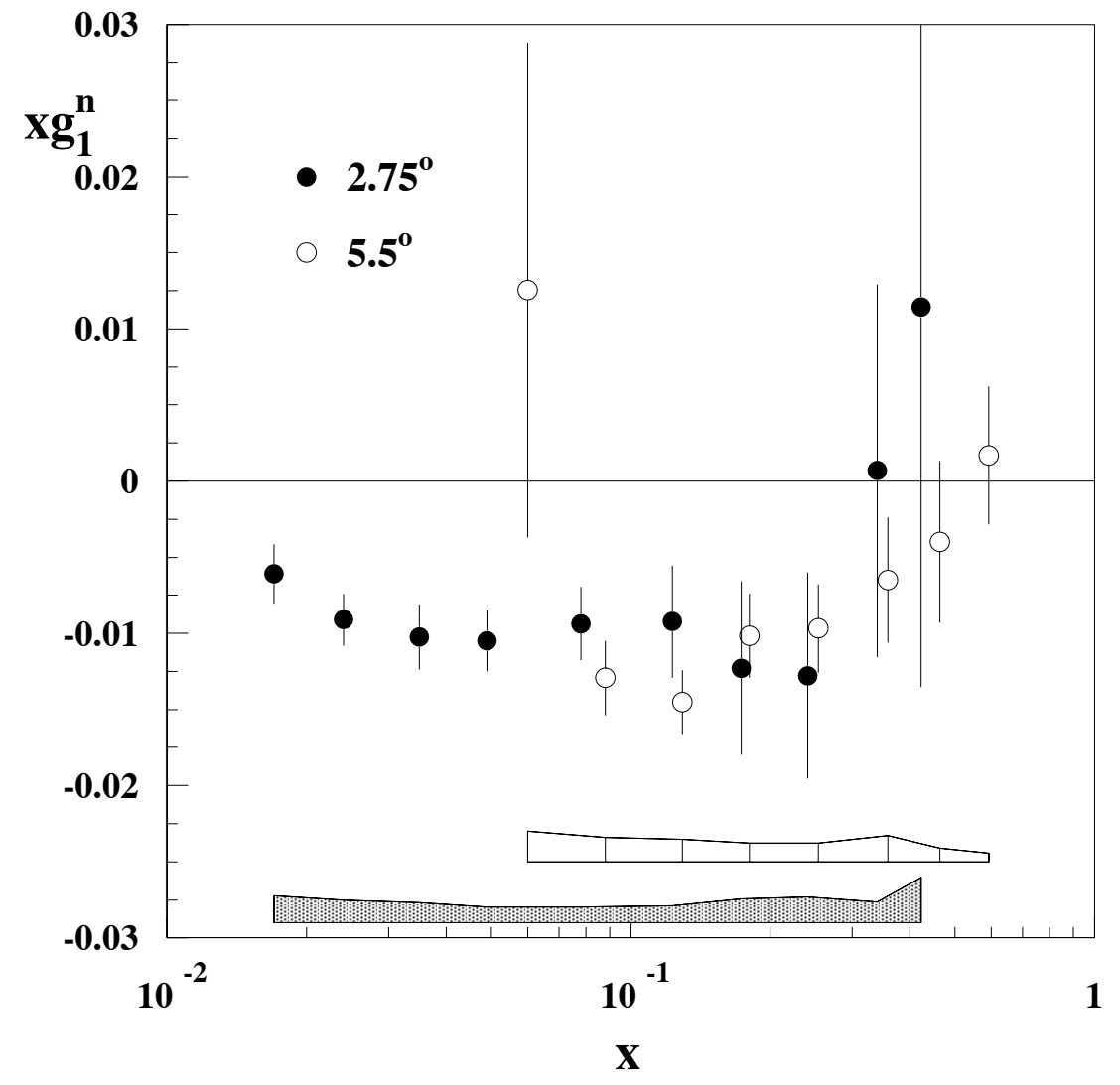

Figure 3.28: The structure function $x g_{1}^{n}$ measured in the $2.75^{\circ}$ (closed circles) and $5.5^{\circ}$ (open circles) spectrometers. The areas at the bottom represent one standard deviation systematic errors.

the measured region from various sources are specified. The biggest uncertainty at the lowest $x$ point is due to the asymmetry of the charge symmetric background $A_{e^{+}}$. As mentioned before, it could be reduced with some theoretical guidance (see section 3.6.2, page 127). The greatest contribution to the uncertainty of the integral comes from the dilution factor and the target polarization. 


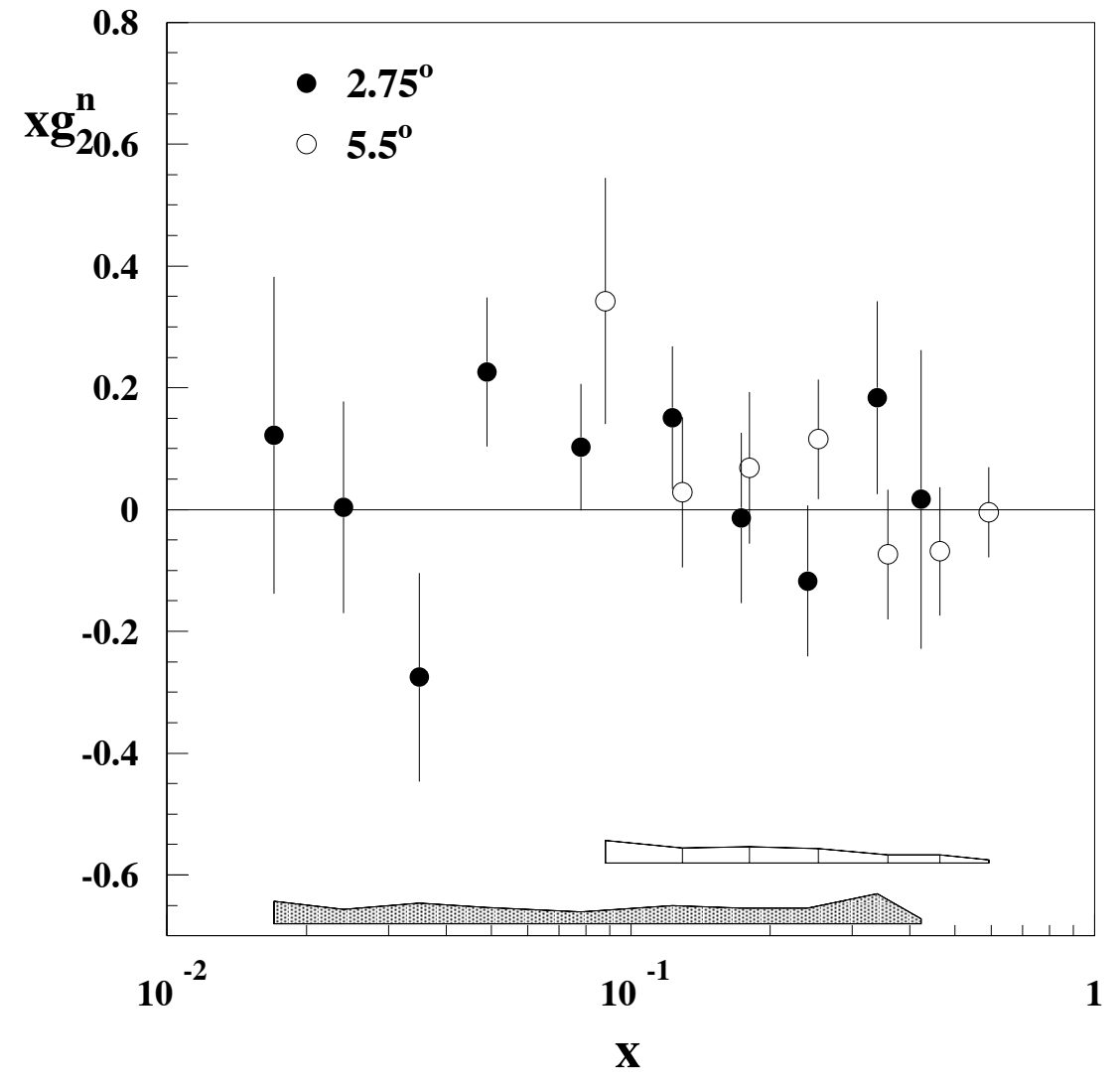

Figure 3.29: The structure function $x g_{2}^{n}$ measured in the $2.75^{\circ}$ and $5.5^{\circ}$ spectrometers. The areas at the bottom represent one standard deviation systematic errors.

\subsection{Discussion of the results}

The E154 results are the most precise results for the polarized neutron spin structure function $g_{1}^{n}$ to date. They are compared to the previous SLAC E142 [17] direct measurement of $g_{1}^{n}$ in fig. 3.31. Both data sets were evolved to the common value of $Q^{2}=5 \mathrm{GeV}^{2}$. The kinematic coverage was extended from 0.03 down to 0.014 in $x$ and from $6 \mathrm{GeV}^{2}$ up to $17 \mathrm{GeV}^{2}$ in $Q^{2}$ (i.e. 


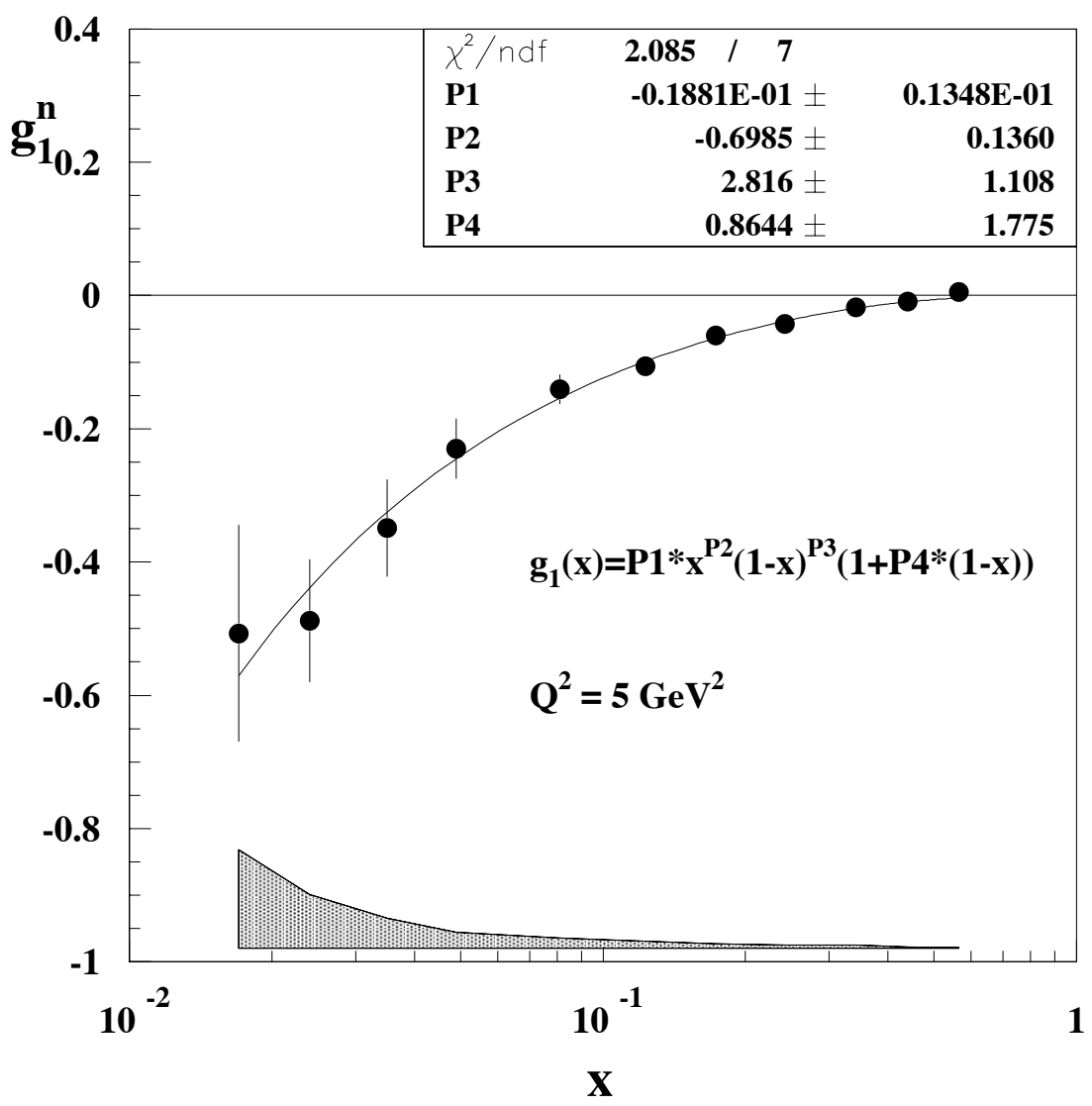

Figure 3.30: The product $g_{1}^{n}$ evaluated at $Q^{2}=5 \mathrm{GeV}^{2}$. Overlaid is a fit to the data. The shaded area represents one standard deviation systematic errors.

from the average $Q^{2}$ of $2 \mathrm{GeV}^{2}$ to $5 \mathrm{GeV}^{2}$ ). The precision was improved by about a factor of 2 . The data agree very well in the common $x$ range. Our results are compared with the data from previous SLAC experiments E142 and E143 [18, 19] in fig 3.32, and with the data of the SMC experiment at CERN $[20,21]$ in fig 3.33, which extends the results to $x \approx 0.003$ but with large uncertainties. 


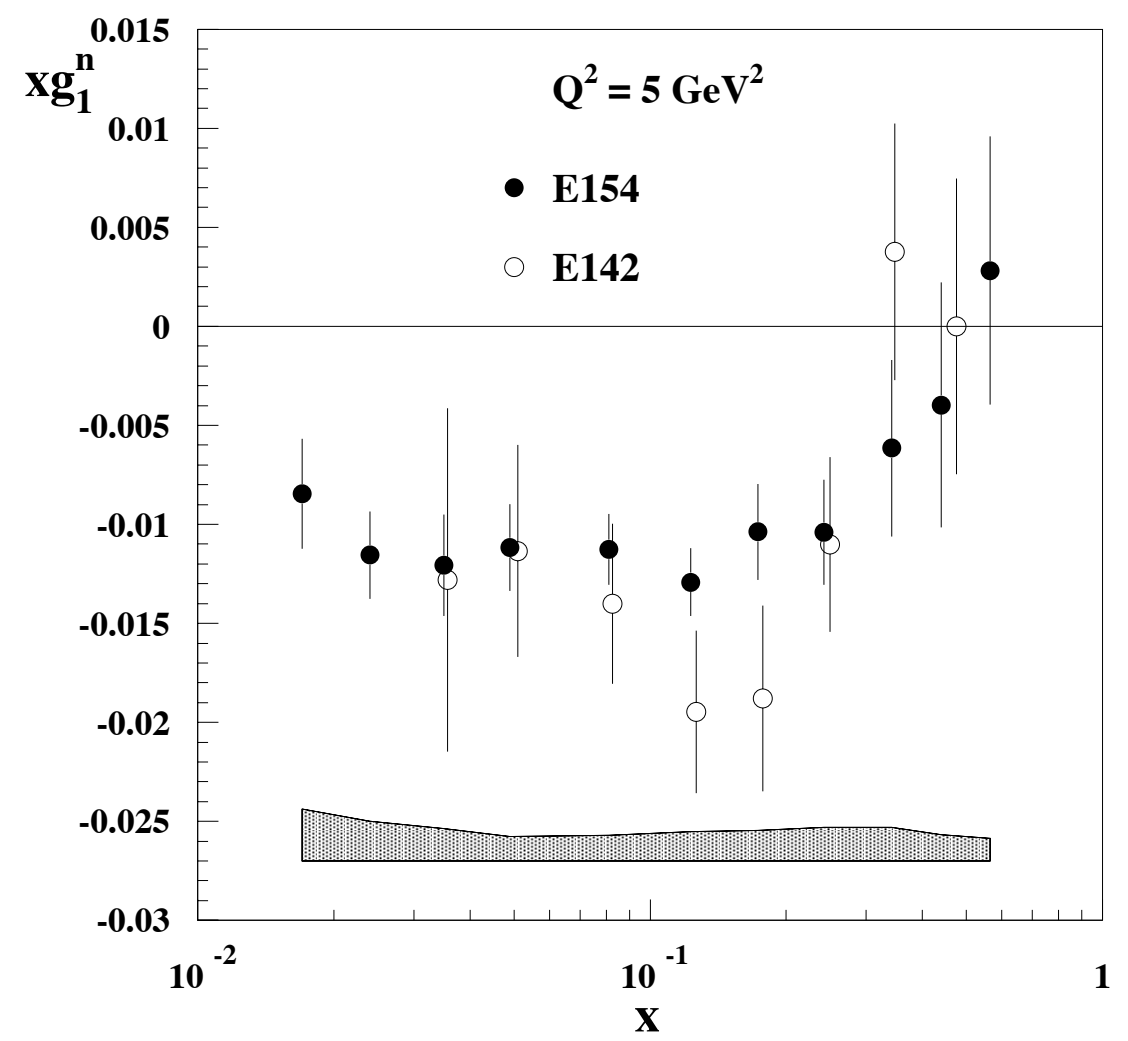

Figure 3.31: The E154 results on the structure function $x g_{1}^{n}$ (closed circles) compared to the E142 (open circles) at $Q^{2}=5 \mathrm{GeV}^{2}$. The E142 data points are slightly offset in $x$ for clarity. The shaded area represents one standard deviation systematic errors of E154.

A noticeable feature of the E154 result is the low $x$ behavior. Contrary to the expected convergence, the $g_{1}^{n}(x)$ is strongly divergent: $g_{1}^{n}(x) \sim x^{-0.7 \pm 0.1}$ as $x \rightarrow 0$, as seen from the fit to all data points in fig. 3.30. The Regge theory predicts, as mentioned in section 1.8 .2 (page 32 ), that

$$
g_{1}(x) \underset{x \rightarrow 0}{\sim} x^{-\alpha},
$$

where $\alpha=-0.5-0$ comes from the $a_{1}$ meson trajectory $[37,57]$. All polarized 


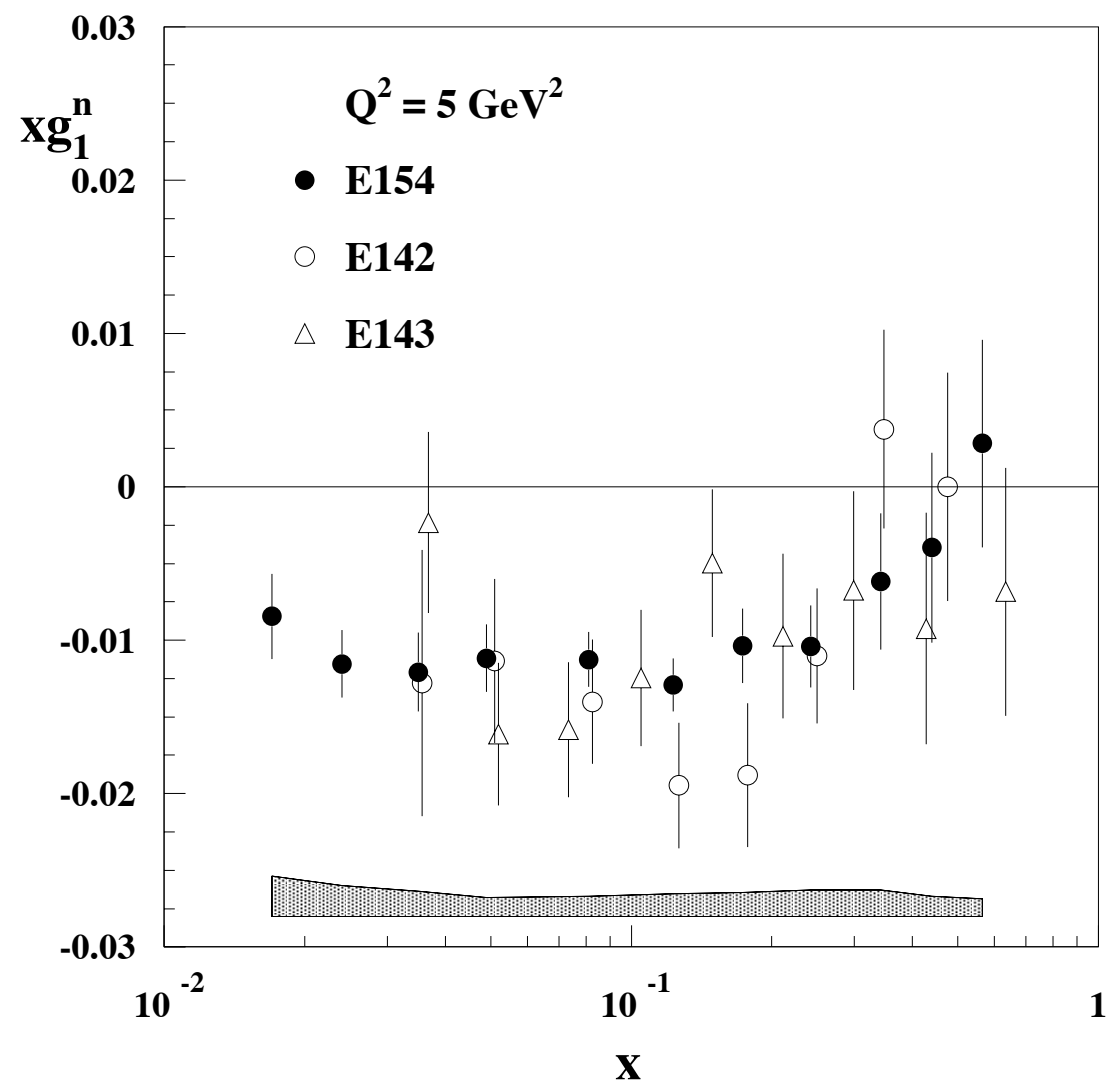

Figure 3.32: Comparison of the E154 results (closed circles) to the E142 (open circles) and E143 (open triangles) data at $Q^{2}=5 \mathrm{GeV}^{2}$. The shaded area represents one standard deviation systematic errors of E154.

nucleon structure experiments $[17,18,19,20,21]$ prior to E154 assumed the Regge low $x$ behavior $[57,125]$ for the extrapolation to $x=0$. The assumption was consistent with the data, and the experiments adopted the possibility of $g_{1}(x) \sim$ const as $x \rightarrow 0$. The Regge theory does not specify the kinematic region in which the asymptotic behavior is applicable. The point $x=0.1$ and $Q^{2}=5 \mathrm{GeV}^{2}$ corresponds to $\sqrt{s} \equiv W \approx 7 \mathrm{GeV}$, and the total 


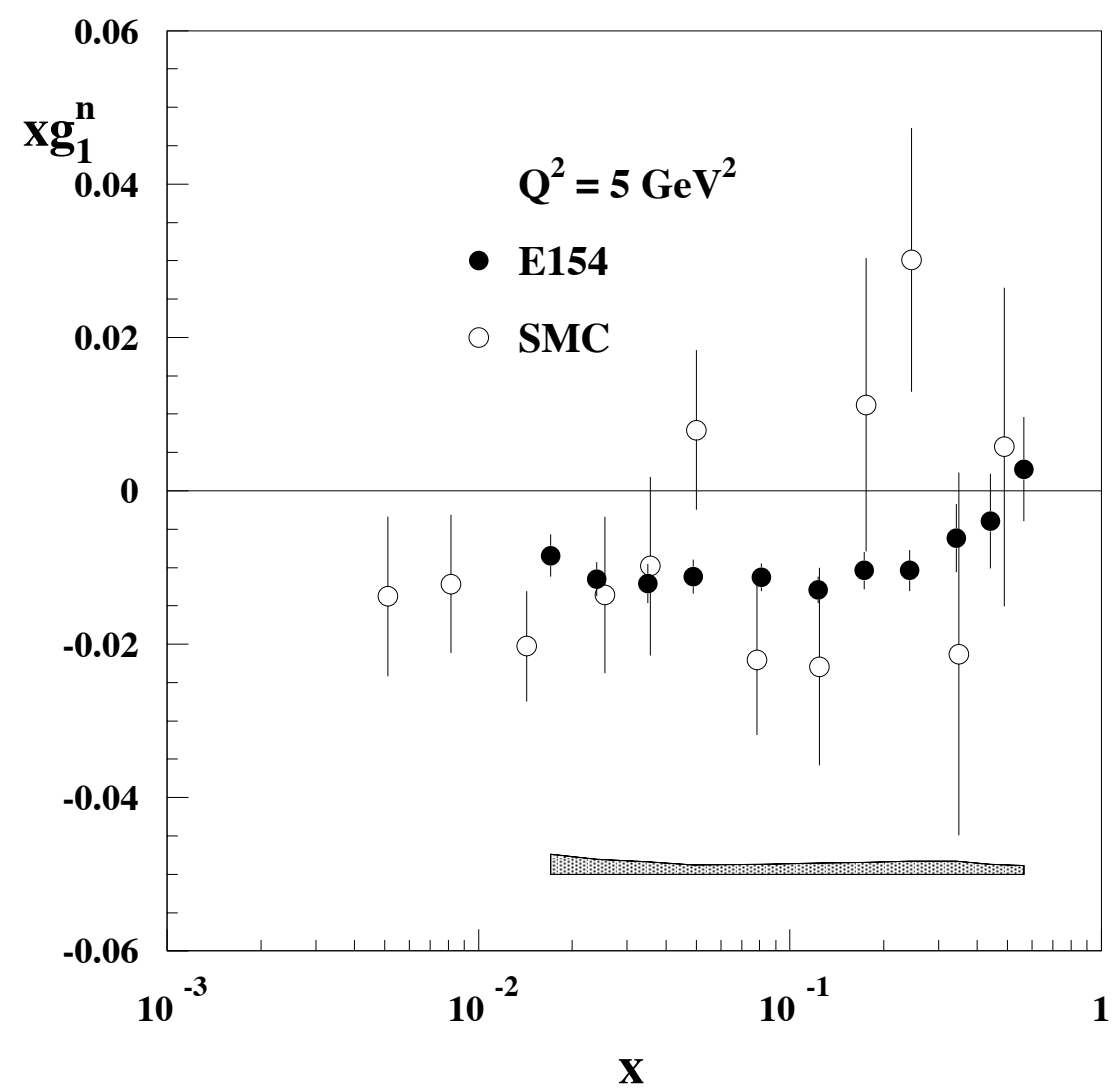

Figure 3.33: Comparison of the E154 results (closed circles) to the SMC data (open circles) at $Q^{2}=5 \mathrm{GeV}^{2}$. The shaded area represents one standard deviation systematic errors of E154.

$\gamma p$ cross sections are well described in that kinematic range by the Regge theory [126]. Also the assumptions $(s / M \approx 49 \mathrm{GeV}) \sim(2 \nu \approx 53 \mathrm{GeV}) \gg$ $\left(\mathrm{Q}^{2} / \mathrm{M} \approx 5 \mathrm{GeV}\right)$ (see eq. 4.31, page 188) that lead from $s$ to $x$ asymptotics are roughly satisfied. Thus one could expect the Regge convergence for $x<$ 0.1. On the other hand the unpolarized data for the $F_{2}$ structure function at $Q^{2} \sim 5 \mathrm{GeV}^{2}$ are well described by pQCD down to $x=10^{-4}$ [127]. The 
constant $g_{1}^{n}(x)$ below $x \leq 0.1$ does not fit the E154 results; $\chi^{2}=24$ for 4 degrees of freedom. One may still fit the three lowest points $(x \leq 0.04)$ to a constant with $\chi^{2}=1.7$ for 2 degrees of freedom. The fit to a form $g_{1}^{n}(x) \sim$ $x^{-\alpha}$ with unconstrained $\alpha$ to the points below $x=0.1$ gives $\alpha=0.9 \pm 0.2$. The fits are shown in fig. 3.34. There are several other predictions for the low

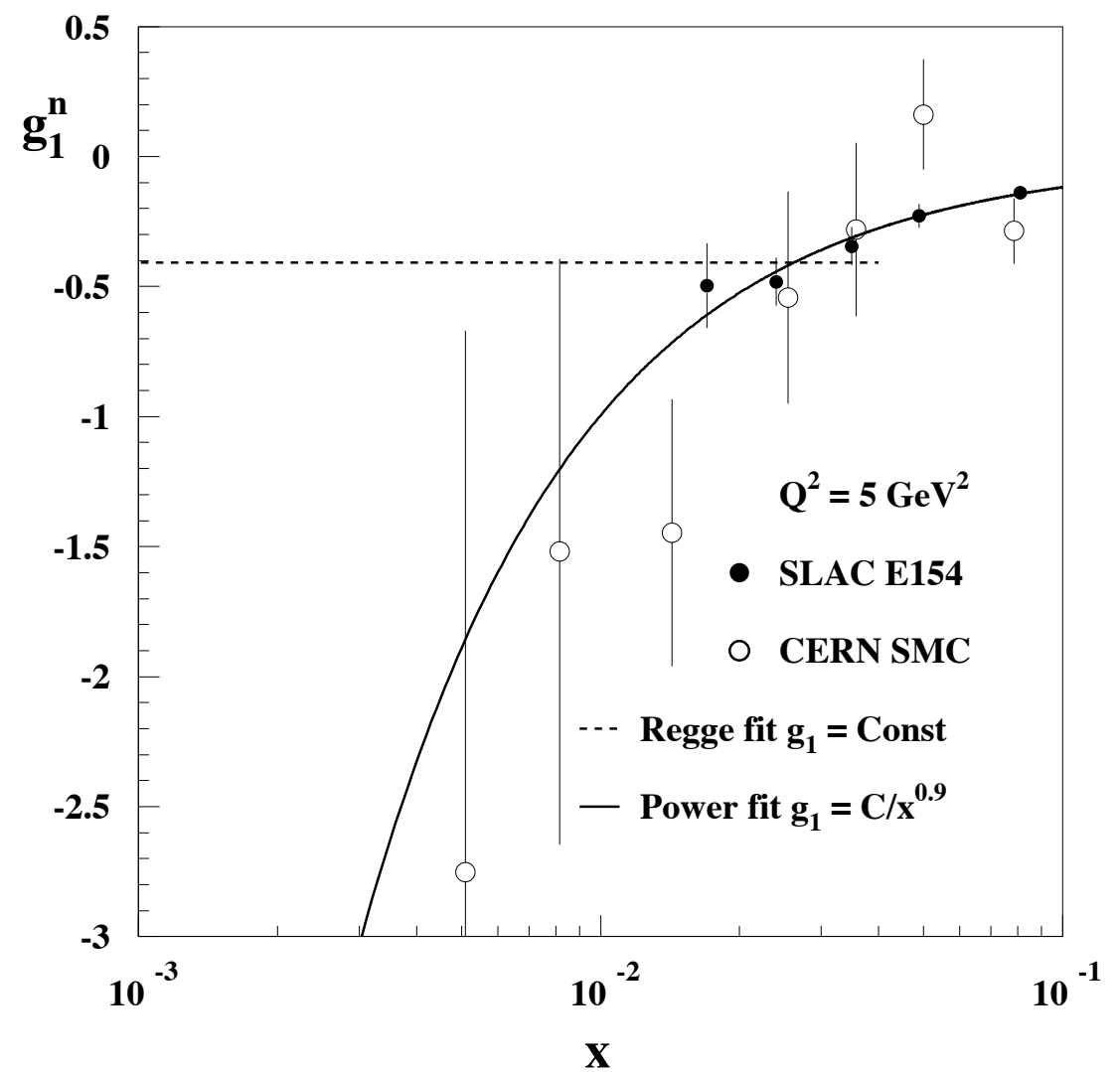

Figure 3.34: Two fits to the low $x$ data of E154. Also the SMC low $x$ data (open circles) are shown.

$x$ behavior of the $g_{1}^{n}$ structure function, see sec. 4.3 .2 (page 187). They lie between the two presented above. A possible interpretation of the strong low 
$x$ behavior is that it is dominated by the sea quarks and gluon contributions which could be very divergent [128].

The integral of $g_{1}^{n}$ in the data range was obtained by summing the values of the structure function in every bin multiplied by the width of the bin. The statistical errors are uncorrelated from bin to bin and thus added in quadrature. Most of the systematic errors are bin-to-bin correlated. Those are added linearly. The uncorrelated systematic errors (on positron, pion asymmetries and $g_{1}^{p}$ ) are added in quadrature. The result for the integral in the measured region is

$$
\int_{0.0135}^{0.7} d x g_{1}^{n}(x)=-0.0360 \pm 0.0039 \pm 0.0045,
$$

where the first error is statistical and the second is systematic.

The large $x$ behavior is predicted from the quark counting rules (see sec. 4.3.1, page 182). The function $g_{1}$ falls off as $(1-x)^{3}$ as $x \rightarrow 1$, or even faster due to the $Q^{2}$ evolution. We assume the $(1-x)^{3}$ dependence and use the last point to set the multiplicative coefficient. That results in the following contribution from the unmeasured high $x$ region:

$$
\int_{0.7}^{1} d x g_{1}^{n}(x)=(0.15 \pm 0.42 \pm 0.04) \times 10^{-3},
$$

where the first error is statistical and the second is systematic.

The integral of $g_{1}^{n}$ from the unmeasured low $x$ region is strongly model dependent. Assuming the Regge behavior $g_{1}^{n}(x)=$ const described above, the value of the integral equals

$$
\int_{0}^{0.0135} d x g_{1}^{n}(x)=-0.0055 \pm 0.0007 \pm 0.0008,
$$


while for the fitted power low behavior $g_{1}^{n}(x) \sim x^{-\alpha}$

$$
\int_{0}^{0.0135} d x g_{1}^{n}(x)=-0.14
$$

The uncertainty in the last result can not be given because the fitted value of $\alpha=0.9 \pm 0.2$ is less than one sigma away from the non-integrable divergence at $x=0$. The different assumptions about the low $x$ asymptotics give drastically different results for the full integral (Ellis-Jaffe sum rule) of the $g_{1}^{n}$ structure function,

$$
\int_{0}^{1} d x g_{1}^{n}(x)=-0.0414 \pm 0.0044 \pm 0.0057
$$

for the Regge behavior, and

$$
\int_{0}^{1} d x g_{1}^{n}(x)=-0.17
$$

for the power low fit. We conclude that the data do not constrain the true asymptotic behavior of $g_{1}^{n}$ at low $x$ making impossible the evaluation of the Ellis-Jaffe sum rule and consequently the quark helicity $\Delta \Sigma=\Delta u+\Delta d+\Delta s$. We illustrate the way the data approach the Ellis Jaffe sum rule in fig. 3.35, where the function $\Gamma_{1}^{n}\left(x_{\text {min }}\right) \equiv \int_{\left(x_{\min }\right.}^{1} d x g_{1}^{n}(x)$ is plotted. The integral in the measured range is almost two times larger than the Ellis-Jaffe prediction.

In a similar manner we plot the integral of $\left(g_{1}^{n}-g_{1}^{p}\right)(x)$ from $x_{\min }$ to 1 as a function of $x_{\min }$. For the proton we use E143 [18] and SMC [20] data. The result is shown in fig. 3.36. Notice that the Bjorken sum rule is almost saturated by the integral over the measured region. We can go further because even if the proton and neutron integrals diverge individually 


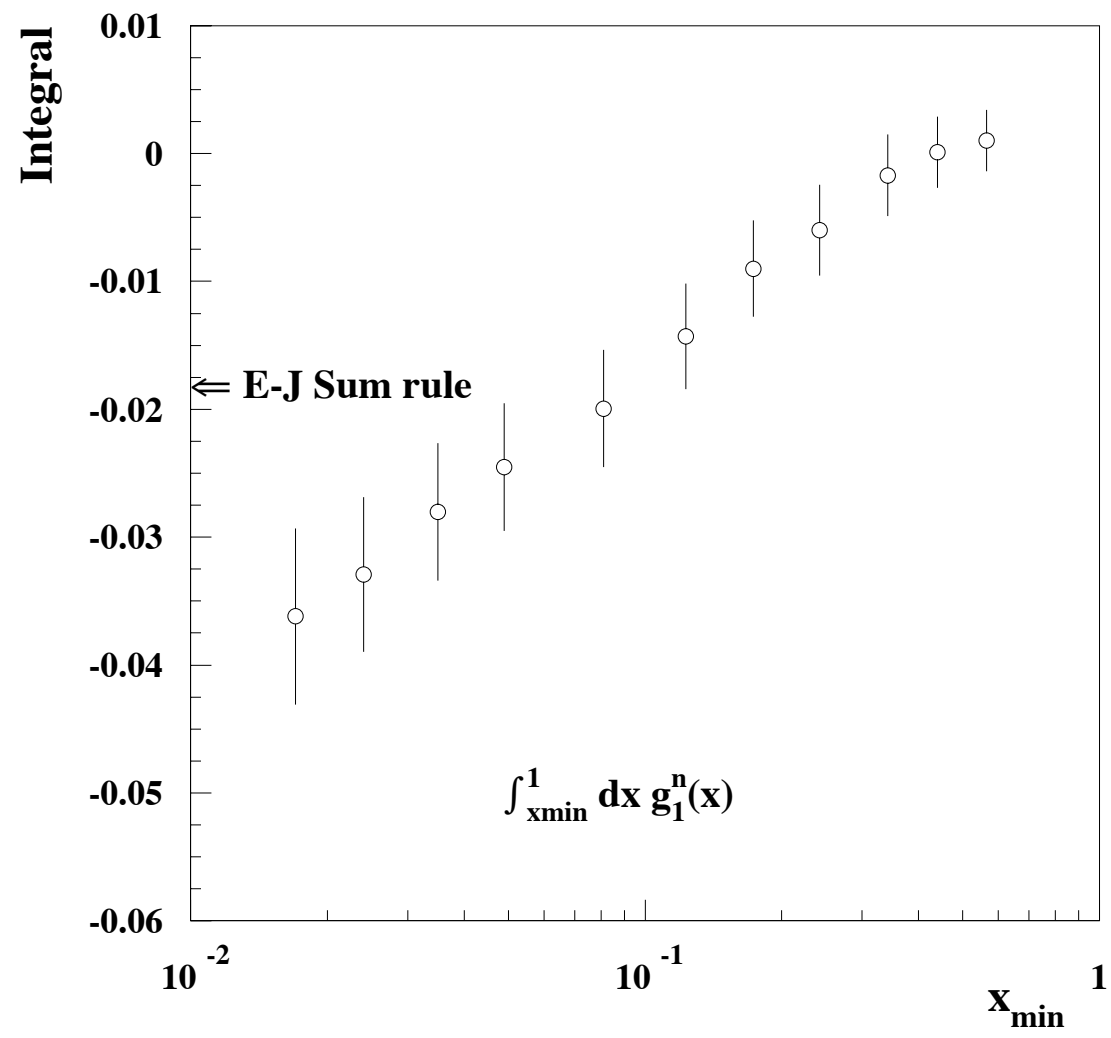

Figure 3.35: The integral of $g_{1}^{n}(x)$ from $x=x_{\min }$ to $x=1$ plotted as a function of $x_{\text {min }}$. The errors are statistical and systematic added in quadrature.

due to the singlet contribution, their difference is purely non-singlet, valence quark distribution (if $\Delta \bar{u}=\Delta \bar{d}$ ). It is expected to be less divergent than the singlet part [129]. The power fit to the low $x$ points below 0.1 gives $\left(g_{1}^{p}-g_{1}^{n}\right)(x) \sim x^{-0.52 \pm 0.11}$, indeed softer asymptotic behavior. The Bjorken sum rule reads

$$
\int_{0}^{1} d x\left(g_{1}^{p}(x)-g_{1}^{n}(x)\right)=0.192 \pm 0.016(\text { stat }) \pm 0.018(\text { syst }),
$$




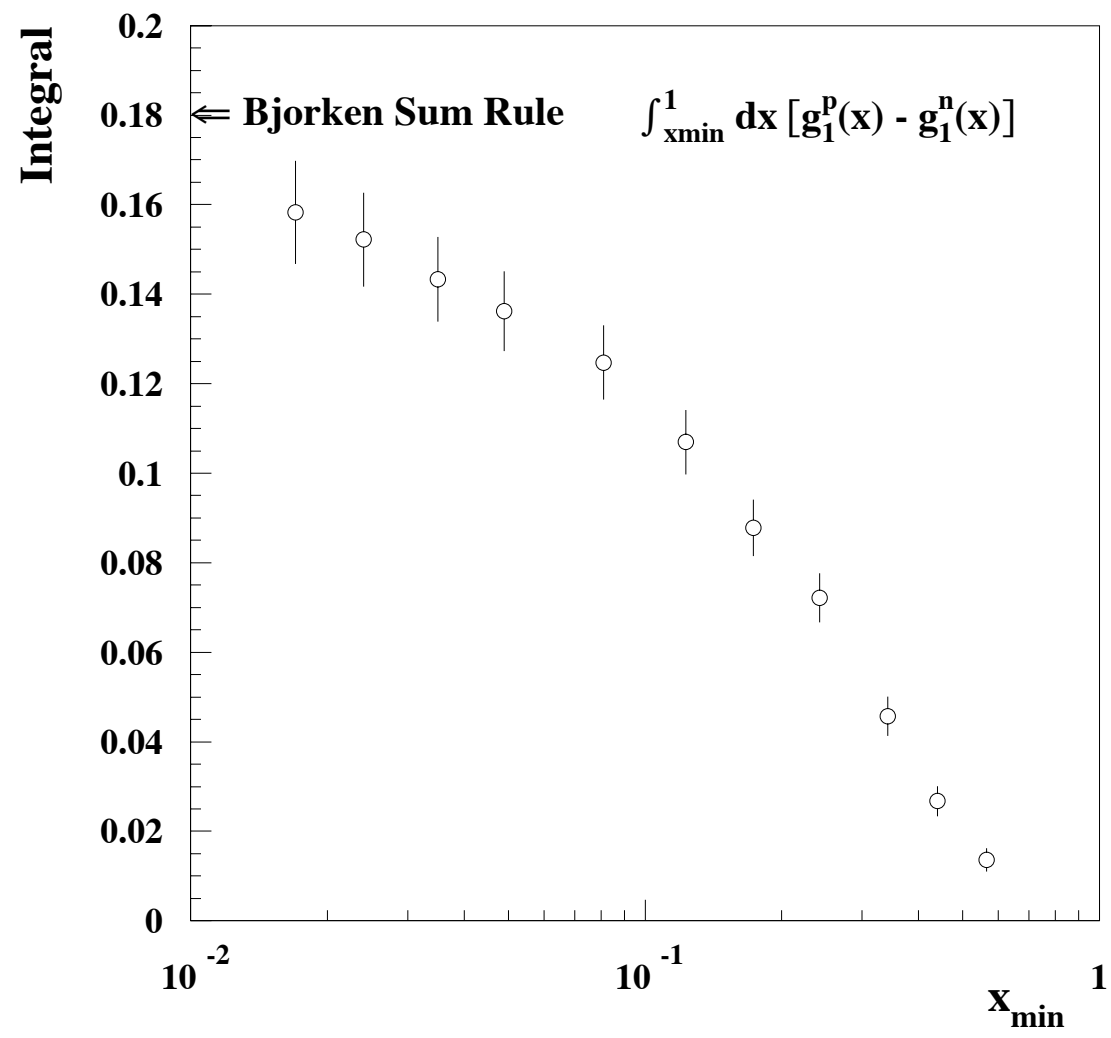

Figure 3.36: The integral of $\left(g_{1}^{n}-g_{1}^{p}\right)(x)$ from $x=x_{\text {min }}$ to $x=1$ plotted as a function of $x_{\text {min }}$. The errors are statistical and systematic, added in quadrature.

in a good agreement with the prediction $\Gamma^{p-n}=0.181 \pm 0.003$ at $5 \mathrm{GeV}^{2}$ with $\alpha_{s}=0.118 \pm 0.003[71]$. 


\section{CHAPTER 4}

\section{GLOBAL FIT}

The kinematic range of the data is constrained by the fixed angle of the spectrometer. That implies that each $x$ point is measured at a different average scale $Q^{2}$. Also the data in the same $x$ bin but different spectrometers are measured at different scales. To evaluate the QCD sum rules, to combine the results from both spectrometers or to compare our results with those obtained by other experiments, one needs to know the values of the structure functions at some arbitrary, fixed $Q^{2}$. Traditionally it has been achieved by evolution based on the assumption that the ratio $g_{1}^{n}\left(x, Q^{2}\right) / F_{1}^{n}\left(x, Q^{2}\right)$ scales, ie. is $Q^{2}$ independent. Although this assumption is consistent within errors with the experimental data, it is incompatible with perturbative QCD (pQCD). It also does not carry a systematic error associated with it, which might become important with the increased accuracy of the measurements. The DIS polarized data on $g^{1}$ structure functions have a reasonable kinematic

range and are more precise, so a global analysis that combines all available 
experimental results in a unified way becomes increasingly meaningful. It allows the spin decomposition of the structure functions into valence and sea quarks and gluons, and not only in terms of the integrals representing the total spin contributions, as conventionally done, but also in terms of the $x$ dependent distributions. The recently calculated next-to-leading order (NLO) polarized anomalous dimensions [130] permit the consistent use of NLO in both the pQCD evolution and the factorization of $g_{1}[131,132,133$, $134]$.

\subsection{Factorization theorem}

The structure function, eg. $g_{1}$, can be expressed in terms of partonic distributions $\Delta p$ via the factorization theorem $[45,135]$ :

$$
g_{1}\left(x, Q^{2}\right)=\sum_{p} C_{p}\left(x, Q^{2} / \mu^{2}, \mu_{f}^{2} / \mu^{2}, \alpha_{s}\left(\mu^{2}\right)\right) \otimes \Delta p\left(x, \mu_{f}, \mu^{2}\right),
$$

with the convolution $\otimes$ defined as

$$
\left(C_{p} \otimes \Delta p\right)\left(x, Q^{2}\right)=\int_{x}^{1} \frac{d y}{y} C_{p}\left(\frac{x}{y}, \ldots\right) \Delta p(y, \ldots) .
$$

The sum is over all partons $p$ : quarks, anti-quarks and gluons. The mass $\mu$ is the renormalization scale present in any perturbative calculation, and $\mu_{f}$ is the factorization scale that defines the separation of the short and long-distance effects. The factorization theorem states that each coefficient function $C_{p}$ corresponds to the hard scattering cross section calculable in pQCD and is infrared safe i.e. independent of long-distance effects, which

are grouped in the partonic distributions $\Delta p$. That implies that $C_{p}$ does 
not depend on the hadron identity, while $\Delta p$ is universal: the same is tru for other structure functions. The coefficient functions $C_{p}$ are referred to as Wilson coefficients. The reason for the name is that the DIS factorization theorem can be formulated in terms of the Operator Product Expansion [136] (OPE).

It is convenient to choose both mass scales equal to the four-momentum transfer: $\mu^{2}=\mu_{f}^{2}=Q^{2}$ and to separate quark distributions $\Delta q$ from antiquark $\Delta \bar{q}$ and the gluon $\Delta G$ distribution. Then factoring out quark charges $e_{q}$ and number of flavors $N_{f}$ from the Wilson coefficients, we arrive at a more familiar QCD improved parton model formulation of the factorization given by eq. (4.1):

$$
g_{1}\left(x, Q^{2}\right)=\frac{1}{2} \sum_{q}^{N_{f}} e_{q}^{2}\left[C_{q} \otimes(\Delta q+\Delta \bar{q})+\frac{1}{N_{f}} C_{G} \otimes \Delta G\right] .
$$

In leading order, $C_{q}\left(x, \alpha_{s}\right)=C_{q}^{(0)}(x)=\delta(1-x)$ and $C_{G}\left(x, \alpha_{s}\right)=C_{G}^{(0)}(x)=0$, in agreement with the simple partonic picture (see eq. (1.55), page 15). In the next-to-leading order (NLO), where

$$
C_{p}\left(x, \alpha_{s}\right)=C_{p}^{(0)}(x)+\frac{\alpha_{s}}{2 \pi} C_{p}^{(1)}(x)
$$

the factorization becomes ambiguous. The finite short-distance parts can be arbitrarily distributed between $C$ 's and $\Delta p$ 's. There is also an arbitrariness of the factorization scale $\mu_{f}$, which divides the perturbative and non-perturbative regions. Propagators that are off-shell by $\mu_{f}^{2}$ and more contribute to the coefficient functions. We will come back to this issue in section 4.6 (page 197). 


\subsection{Evolution}

According to the factorization theorem the measurable quantities, eg. structure functions, do not depend on the factorization scale $\mu_{f}$. This leads to predictions of the way in which the partonic distributions depend on this scale. The dependence, called evolution, is the remarkable result of pQCD that connects experimental data obtained at different $Q^{2}$ s. It is described by the Dokshitzer-Gribov-Lipatov-Altarelli-Parisi (DGLAP) [137, 138] equations:

$$
\begin{aligned}
Q^{2} \frac{d}{d Q^{2}} \Delta q_{N S}^{\eta} & =\frac{\alpha_{s}\left(Q^{2}\right)}{2 \pi} P_{N S}^{\eta} \otimes \Delta q_{N S}^{\eta}, \quad \eta= \pm 1, \\
Q^{2} \frac{d}{d Q^{2}}\left(\begin{array}{c}
\Delta \Sigma \\
\Delta G
\end{array}\right) & =\frac{\alpha_{s}\left(Q^{2}\right)}{2 \pi}\left(\begin{array}{cc}
P_{q q} & P_{q G} \\
P_{G q} & P_{G G}
\end{array}\right) \otimes\left(\begin{array}{c}
\Delta \Sigma \\
\Delta G
\end{array}\right),
\end{aligned}
$$

where the index NS stands for the non-singlet quark distributions, i.e. the valence distributions $(\eta=1)$

$$
\left.\begin{array}{rl}
\Delta u_{V}\left(x, Q^{2}\right) & \equiv \Delta u-\Delta \bar{u} \\
\Delta d_{V}\left(x, Q^{2}\right) & \equiv \Delta d-\Delta \bar{d},
\end{array}\right\} \quad \Delta q_{N S}^{\eta=+1}
$$

and the $S U(3)_{\text {flavor non-singlet combinations }(\eta=-1)}$

$$
\left.\begin{array}{rl}
\Delta q_{3}\left(x, Q^{2}\right) & \equiv(\Delta u+\Delta \bar{u})-(\Delta d+\Delta \bar{d}), \\
\Delta q_{8}\left(x, Q^{2}\right) & \equiv(\Delta u+\Delta \bar{u})+(\Delta d+\Delta \bar{d})-2(\Delta s+\Delta \bar{s}),
\end{array}\right\} \quad \Delta q_{N S}^{\eta=-1} .
$$

and where $\Delta \Sigma$ stands for the $S U(3)_{\text {flavor }}$ singlet combination

$$
\Delta \Sigma\left(x, Q^{2}\right)=(\Delta u+\Delta \bar{u})+(\Delta d+\Delta \bar{d})+(\Delta s+\Delta \bar{s}),
$$


and $P$ for the polarized splitting functions. In the leading order the splitting functions have the form $[137,138]$

$$
\begin{aligned}
P_{N S}^{\eta(0)}(z) & =P_{q q}^{(0)}(z)=\frac{4}{3}\left[\frac{1+z^{2}}{(1-z)_{+}}+\frac{3}{2} \delta(z-1)\right], \\
P_{q G}^{(0)}(z) & =\frac{1}{2}\left[z^{2}-(1-z)^{2}\right], \\
P_{G q}^{(0)}(z) & =\frac{4}{3}\left[\frac{1-(1-z)^{2}}{z}\right], \\
P_{G G}^{(0)}(z) & =3\left[\left(1+z^{4}\right)\left(\frac{1}{z}+\frac{1}{(1-z)_{+}}\right)-\frac{(1-z)^{3}}{z}+\left(\frac{11}{6}-\frac{N_{f}}{9}\right) \delta(z-1)\right],
\end{aligned}
$$

where $N_{f}$ is the number of active flavors and the $1 /(1-z)_{+}$regularization means that

$$
\int_{0}^{1} d z \frac{f(z)}{(1-z)_{+}} \equiv \int_{0}^{1} d z \frac{f(z)-f(1)}{(1-z)},
$$

and $1 /(1-z)_{+}=1 /(1-z)$ for $z<1$. The splitting functions have recently been calculated in the NLO in the $\overline{\mathrm{MS}}$ factorization scheme in [130] and are given in the Appendix B.2 (page 279).

The parton model provides us with an intuitive interpretation of the evolution. Each parton is surrounded by a cloud of virtual gluons and quarkanti-quark pairs. When we probe a nucleon with low resolution, we cannot resolve that structure. In particular we see some probability $q\left(Q^{2}, x\right)$ of finding a "constituent" quark with a fraction $\mathrm{x}$ of the nucleon momentum. By increasing the probing scale we can detect the quark, eg. after the emission of a gluon which reduced the initial momentum y by a factor $z$ to the measured value $\mathrm{x}$. That radiation happens with probability $\left(\alpha_{s}\left(Q^{2}\right) /(2 \pi)\right) * P(z)$, which is a perturbatively calculable hard scattering cross section. The situation is shown in fig. 4.1. We can compute the change of the probability due 


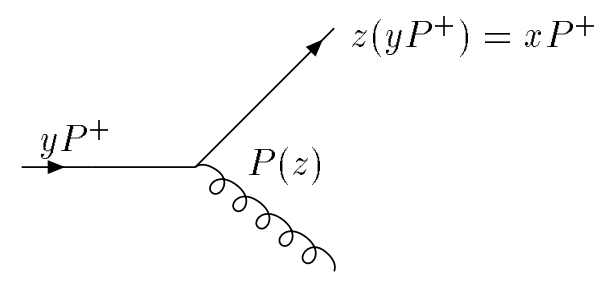

Figure 4.1: Change of the partonic probability due to the radiation of a gluon. $P^{+}$is the light-cone + component of the nucleon momentum.

to a change in the resolution by summing over all momenta in the described process:

$$
\delta q(x, t)=\delta t \int_{0}^{1} d y \int_{0}^{1} d z \delta(x-z y) \frac{\alpha_{s}(t)}{2 \pi} P(z) q(y, t)
$$

where $t=\log \left(Q^{2} / \Lambda^{2}\right)$. After integrating over $z$, we come to the evolution equation (see eq. (4.5)),

$$
\frac{d}{d t} q(x, t)=\frac{\alpha_{s}(t)}{2 \pi} \int_{x}^{1} \frac{d y}{y} P\left(\frac{x}{y}\right) q(y, t) .
$$

\subsection{Initial distributions}

The factorization theorem enables us to construct structure functions from the Wilson coefficients and partonic distributions. The distributions summarize long-distance correlations and thus cannot be obtained perturbatively. We have to model them, ie. assume some functional $x$ dependence or shape $p\left(x, Q_{\circ}^{2}\right)=p(x)$ at a certain initial scale $Q_{\circ}^{2}$. Then we can connect 
those initial distributions via pQCD evolution and the factorization theorem with the experimental measurements at different momenta transfer $Q^{2}$. Here pQCD provides us with some guidance about the asymptotic behavior of $p(x)$ at high $x \sim 1$, and the Regge phenomenology suggests the low $x \sim 0$ behavior.

The QCD analysis of the high $x$ endpoint behavior is most conveniently formulated in the light-cone formalism (see ref. [139, 140] for an overview).

\subsubsection{High $x$ asymptotics}

The quark distributions $q(x, \lambda)$ are probability densities determined by the light-cone wave-functions $\psi_{n}\left(x_{i}, k_{\perp i}, \lambda_{i}\right)$ of $\mathrm{n}$ constituents with the helicities $\lambda_{i}$, momenta fractions $x_{i}$, and perpendicular momenta $k_{\perp i}$ which add up to the total momentum of the nucleon: $\sum_{i=1}^{n} x_{i}=1$ and $\sum_{i=1}^{n} k_{\perp i}=0_{\perp}$. The square of the invariant mass of such an n-particle state is $\mathcal{M}_{n}^{2}=\sum_{i=1}^{n}\left(k_{\perp i}^{2}+\right.$ $\left.m_{i}^{2}\right) / x_{i}$. If one quark carries nearly all of the light-cone momentum $x \sim 1$ of the nucleon, then the remaining constituents have $x_{i} \approx(1-x) \sim 0$, and the total invariant mass $\mathcal{M}_{n}^{2} \sim 1 /(1-x)$ is far off-shell. Assuming that the nucleon bound state wave function is dominated by the lowest invariant mass partonic configurations, the kinematic regime $x \sim 1$ is achieved only by the exchange of hard gluons. Such processes can be treated perturbatively $[140]$ in the following way: The nucleon wave-function $\psi\left(x_{i}, k_{\perp i}\right)$ with one of the quarks carrying nearly all momentum is a convolution of the nucleon bound state wave-function $\psi\left(y_{i}, p_{\perp i}\right)$ and the hard scattering amplitude 
$A\left(y_{i}, p_{\perp i} ; x_{i}, k_{\perp i}\right)$

$$
\psi\left(x_{i}, k_{\perp i}\right)=\int\left[d y_{i}\right]\left[d p_{\perp i}\right] \psi\left(y_{i}, p_{\perp i}\right) A\left(y_{i}, p_{\perp i} ; x_{i}, k_{\perp i}\right),
$$

as shown in fig. 4.2. The quark distribution $q(x)$ is simply the probability

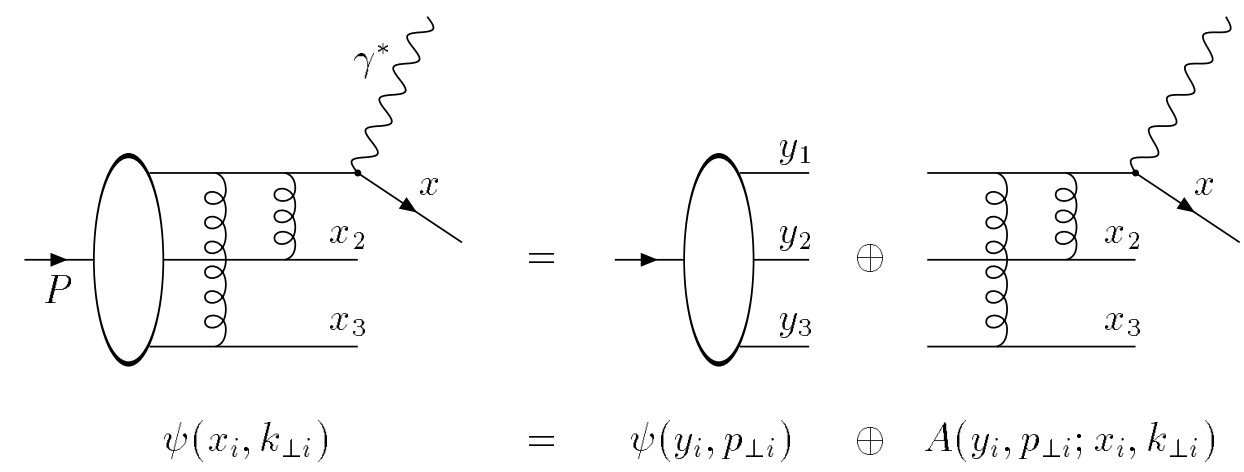

Figure 4.2: Leading-order diagram for the amplitude $\psi(x)$ at $x \sim 1$. Here the convolution $\oplus$ means: $\psi \oplus A=\int\left[d y_{i}\right]\left[d p_{\perp i}\right] \psi A$.

density obtained from the wave-function $\psi\left(x_{i}, k_{\perp i}\right)$ :

$$
q(x)=\int \prod d x_{i} \delta\left(\sum x_{i}-1\right) \delta\left(x-x_{1}\right) \int\left[d k_{\perp i}\right]\left|\psi\left(x_{i}, k_{\perp i}\right)\right|^{2} .
$$

The endpoint $x \sim 1$ behavior comes from the hard scattering amplitude $A$ and from phase space, i.e. integration over $x_{i}$ 's. We count the $(1-x)$ powers. First for the amplitude $A$ :

$(1-x)^{2(n-1)}$ : each gluon and on-shell fermion propagator contains (momentum transferred $)^{2}$ in the denominator, and (momentum transferred) ${ }^{2}$ $\sim k_{\perp}^{2} /(1-x)$

$(1-x)^{-(n-1)}$ : each set of Dirac matrix elements in the numerator from every quark with a single gluon attachment contributes a factor $1 /(1-x)$; 
$(1-x)^{-\frac{1}{2}((n-1)-2|\Delta \lambda|)}:$ here $\Delta \lambda$ is the difference between helicity of the nucleon and the struck quark, i.e. $\Delta \lambda=0$ if the helicities are aligned or $\Delta \lambda=1$ if anti-aligned. This factor comes from the instantaneous part of the off-shell fermion propagator (off-shell = on-shell + instantaneous). This part behaves like $\gamma^{+} /(1-x)$. Because $\left(\gamma^{+}\right)^{2}=0$ and $\gamma^{+} \gamma^{R, L} \gamma^{+}=0$, it is sensitive to the quark helicities. Remembering that the valance quark helicities sum to the parent nucleon helicity, one finds that $\frac{1}{2}((n-1)-2|\Delta \lambda|)$ is the maximum number of properly aligned quark pairs giving non-vanishing contribution.

And for the phase space factor:

$(1-x)^{n-2}:$ comes from $\mathrm{n}$ integrals over $x_{i}$ with two $\delta$ functions.

Thus we get the classical power counting result [58]:

$$
q(x, \lambda) \underset{x \rightarrow 1}{\sim}(\text { phase space })|A|^{2} \sim(1-x)^{2 n-3+2|\Delta \lambda|} .
$$

This counting rule says that the Fock state with the minimum number $n$ of constituents gives the leading contribution; all higher states produce a faster fall off of the wave-function. Also it reflects the helicity retention property, namely, that the quark that carries most of the nucleon's momentum also tends to carry its helicity. In other words

$$
\lim _{x \rightarrow 1} \frac{\Delta q(x)}{q(x)}=1,
$$

where $\Delta q(x)=q(x,+)-q(x,-)$ and $q(x)=q(x,+)+q(x,-)$ with $\lambda=+$ for aligned helicities of the quark and nucleon and $\lambda=-$ for anti-aligned 
helicities. The anti-aligned quark distributions are suppressed by an additional factor $(1-x)^{2}$. We can combine the counting rule with the splitting functions, eq. (4.6), $P_{G q}(q \rightarrow q G)$ and $P_{q G}(G \rightarrow q \bar{q})$ to predict the $x \sim 1$ behavior of the gluon $G(x)$ and sea quark $s(x)$ distributions. The splitting also reflects the helicity retention property. The sign of the helicity of the parent parton is transfered to the constituent with the largest momentum fraction. Again the opposite helicity is suppressed by $(1-x)^{2}$. Thus equation (4.17) is valid for any parton: valence quarks, sea quarks or gluons,

$$
\lim _{x \rightarrow 1} \frac{\Delta p(x)}{p(x)}=1
$$

where $p=q, \bar{q}$ or $G$. For the helicity aligned gluon the splitting function $P_{G} q(x,+)=\frac{4}{3} \frac{1}{x}$ while for the antiparallel alignment $P_{G} q(x,-)=\frac{4}{3} \frac{(1-x)^{2}}{x}$ (note that $\left.P_{G} q(x)=P_{G} q(x,+)-P_{G} q(x,-)\right)$. When those splitting functions are convoluted with quark distributions asymptotically described by eq. (4.16), they give the leading behavior of the gluonic distributions,

$$
G(x, \lambda) \underset{x \rightarrow 1}{\sim}(1-x)^{2 n-2+2|\Delta \lambda|},
$$

which are suppressed by at least one additional power of $1-x$ in comparison with the quark distributions. Gluons can decay into sea quarks, and their distribution convoluted with the splitting functions $P_{q} G(x, \pm)$ gives one more power of $1-x$ in the extrinsic sea $s(x)$ quark distributions:

$$
s(x, \lambda) \underset{x \rightarrow 1}{\sim}(1-x)^{2 n-1+|\Delta \lambda|} .
$$


In the case of the neutron the minimum number of constituent quarks is three $(n=3)$, so the valence quark distributions have the behavior

$$
q(x,+) \underset{x \rightarrow 1}{\sim}(1-x)^{3} \text { and } q(x,-) \underset{x \rightarrow 1}{\sim}(1-x)^{5}
$$

the gluon distributions

$$
G(x,+) \underset{x \rightarrow 1}{\sim}(1-x)^{4} \text { and } G(x,-) \underset{x \rightarrow 1}{\sim}(1-x)^{6},
$$

the extrinsic sea quarks e.g. strange quarks

$$
s(x,+) \underset{x \rightarrow 1}{\sim}(1-x)^{5} \text { and } s(x,-) \underset{x \rightarrow 1}{\sim}(1-x)^{7},
$$

and finally the intrinsic strange quark distributions which are associated with the Fock state and have at least five partons:

$$
s_{i}(x,+) \underset{x \rightarrow 1}{\sim}(1-x)^{7} \text { and } s_{i}(x,-) \underset{x \rightarrow 1}{\sim}(1-x)^{9} .
$$

The counting rule derived for the quark and gluon distributions is valid at the low momentum transfer $Q_{0} \sim \Lambda_{Q C D}$ at which the physics of the nucleon is governed by the bound state wave-function rather than the radiative processes associated with the evolution. At higher $Q^{2}$ the radiation from the struck quark increases the initial $(1-x)^{p}$ power fall-off by

$$
\Delta p=\frac{4 C_{F}}{\beta_{0}} \log \left(\frac{\log \left(Q^{2} / \Lambda^{2}\right)}{\log \left(Q_{0}^{2} / \Lambda^{2}\right)}\right),
$$

where $C_{F}=4 / 3$ and $\beta_{0}=11-(2 / 3) N_{f}$.

The continuity between the elastic and inelastic physics at fixed invariant mass [141], sometimes called the dual description of resonance production, 
predicts a correspondence between the $x \sim 1$ behavior of the quark helicity distributions $q(x)$ and the $Q^{2}$ dependence of the form factors $F\left(Q^{2}\right)[142]$ at large $Q^{2}$,

$$
\left(q(x) \underset{x \sim 1}{\sim}(1-x)^{2 m-1}\right) \Longleftrightarrow\left(F\left(Q^{2}\right) \underset{\text { large } Q^{2}}{\sim}\left(1 / Q^{2}\right)^{m}\right) .
$$

The SLAC measurements [143] of the elastic electron-proton scattering are compatible with the pQCD predictions for both the helicity conserving $F_{1}\left(Q^{2}\right)$ and the helicity changing $F_{2}\left(Q^{2}\right)$ form-factors. The products $Q^{4} F_{1}\left(Q^{2}\right)$ and

$Q^{6} F_{2}\left(Q^{2}\right)$ become approximately constant at large $Q^{2}$. That corresponds to the helicity parallel distribution $q(x,+) \sim(1-x)^{3}$ and the helicity antiparallel distribution $q(x,-) \sim(1-x)^{5}$ at $x \sim 1$, in agreement with the counting rules.

\subsubsection{Low $x$ phenomenology}

The asymptotics of the structure functions in the region of $x \sim 0$ is suggested by the Regge pole formulas [144] that come from the analysis of two body invariant scattering amplitude $A(s, t)$ in the physical t-channel. The amplitude, written as a partial-wave expansion sum, can be converted via the Watson-Sommerfeld transformation to an integral in the complex angular momentum $\alpha(t)$ plane. Assuming analyticity in s and in $\alpha(t)$, one can show that the large s limit (crossed physical s-channel) is given by

$$
A(s, t) \underset{\text { large } s}{\sim}\left(\sum_{i}+\int_{i}\right) \beta_{i}(t) s^{\alpha_{i}(t)},
$$

where the sum is over Regge poles (isolated poles in the complex angular momentum plane) and the integral over Regge cuts. In the limit $s \rightarrow \infty$ the 
largest (rightmost) $\alpha$ (or a few largest) dominate, and in case of a pole

$$
A(s, t) \underset{s \rightarrow \infty}{\sim} \beta(t) s^{\alpha(t)} .
$$

This corresponds to the exchange of all t-channel particles and resonances, each having a pole at $t=M^{2}(\alpha(t))$ belonging to the same Regge trajectory ${ }^{1}$. The optical theorem relates the imaginary part of the forward $(t=0)$ scattering amplitude $A^{a b \rightarrow c d}(s, t=0)$ to the total cross section $\sigma^{a b \rightarrow c d}(s)$ for the process $a b \rightarrow c d$,

$$
\operatorname{Im}\left(A^{a b \rightarrow c d}(s, t=0)\right) \sim s \sigma^{a b \rightarrow c d}(s) .
$$

Thus from eq. (4.28)

$$
\sigma^{a b \rightarrow c d}(s) \sim s^{\alpha(0)-1} .
$$

The energy of the virtual photon-proton scattering in the CMS equals

$$
s \equiv W^{2}=(P+q)^{2}=M^{2}-Q^{2}+2 M \nu,
$$

where $2 M \nu=Q^{2} / x$. The Regge $s \rightarrow \infty$ limit at fixed $Q^{2}$ implies $\nu \rightarrow \infty$ or $x \rightarrow 0$. The structure function $g_{1}$ is given in terms of the total virtual photo-absorption cross sections by $g_{1} \sim \nu\left(\sigma_{1 / 2}-\sigma_{3 / 2}\right)$ (see eq. (1.26), (1.27), (1.30), page 10). Assuming single Regge pole dominance, eq. (4.30), for $\sigma_{1 / 2}$ and $\sigma_{3 / 2}$ we find the high energy transfer behavior:

$$
g_{1}\left(\nu, Q^{2}\right) \underset{\nu \rightarrow \infty}{\sim} \beta\left(Q^{2}\right) \nu^{\alpha(0)},
$$

\footnotetext{
${ }^{1}$ Loosely speaking a Regge trajectory consists of the lowest bound state and its rotational excitations of the same parity. We denote the mass by $M$ and the spin by $J$. It turns out that a single trajectory satisfies the linear relation $J(M)=\alpha_{0}+\alpha^{\prime} M^{2}$, with a trajectory dependent J-intercept $\alpha_{0}$ and the universal slope $\alpha^{\prime}$. If we substitute $M^{2}=t$ and $J(M)=\alpha(t)$ in this relation, we find a linear dependence of $\alpha(t)$ on $t$ called a Regge trajectory.
} 
or equivalently, the low $\mathrm{x}$ behavior,

$$
g_{1}\left(x, Q^{2}\right) \underset{x \rightarrow 0}{\sim} \beta^{\prime}\left(Q^{2}\right) x^{-\alpha(0)} .
$$

Let us point out that the above formulae are not valid in the full deep inelastic region, i.e. in the Bjorken limit where both $\nu$ and $Q^{2} \rightarrow \infty$ with $x$ fixed. In that limit $g_{1}\left(\nu, Q^{2}\right)$ becomes a function of $x$ only and, as eq. (4.32) dictates, $\beta\left(Q^{2}\right)\left(Q^{2}\right)^{\alpha(0)} \sim$ const, so $\beta\left(Q^{2}\right) \sim\left(Q^{2}\right)^{-\alpha(0)}$. Now $\nu$ is of the same order as $Q^{2}$ so $\beta\left(Q^{2}\right) \sim \nu^{-\alpha(0)}$. But this means (see eq. (4.32)) the contributions from all Regge poles are of the same order of magnitude. Thus we expect the Regge description to be valid at low $x$ and low $Q^{2}$ (in comparison to $\nu$ ), and assume a smooth transition from that region to the deep inelastic region. A quantitative estimate of how low $x$ and $Q^{2}$ should be is not predicted by this analysis. It has been shown in $[37,57]$ that the poles of the $a_{1}$ meson trajectory, with the intercept $\alpha_{a_{1}}(0)=-0.14 \pm 0.20$, give the leading behavior of the $g_{1}$ function,

$$
g_{1}(x) \underset{x \sim 0}{\sim} x^{0.14}
$$

Traditionally low x extrapolations of $g_{1}(x)$ with $\alpha(0)=0$ have been performed with the uncertainty in the parameter reflected by the extrapolation uncertainty.

There are also other predictions. In [145] it is argued that a two-Pomeron cut gives a singular contribution ${ }^{2}$ to the singlet part of $g_{1}$,

$$
g_{1}^{S}(x) \sim \frac{1}{x \log ^{2}(x)}
$$

\footnotetext{
${ }^{2}$ There exist also counter-arguments [37].
} 
as $x \rightarrow 0$. The Pomeron modeled as the exchange of two non-perturbative gluons [146] gives

$$
g_{1}(x) \sim(1+2 \log (x))
$$

The analysis of the three-gluon vertex [147] produces

$$
g_{1}(x) \sim\left(F_{2}(x)\right)^{\sqrt{2}}
$$

The recently performed re-summation [128, 129] of double logarithmic terms of the form $\left(\alpha_{s} \log ^{2}(1 / x)\right)^{k}$ in the anomalous dimensions leads to an even more singular behavior:

$$
g_{1}^{N S}(x) \sim x^{-0.4}, \quad g_{1}^{S}(x) \sim \frac{1}{x \log ^{3 / 2}\left(\frac{1}{x}\right)} .
$$

We do not have enough polarized data to distinguish between these different possibilities. However we can get some hints from the vast amount of unpolarized experimental data. For many elastic reactions the total cross section is in excellent agreement with the Regge theory, for example $p p, p \bar{p}$, $\pi^{ \pm} p[148]$ or $\gamma p[126]$ scattering. The recent analysis [127] of $F_{2}(x)$ structure function down to $x \gtrsim 3 \cdot 10^{-6}$ and $Q^{2} \gtrsim 0.16 \mathrm{GeV}^{2}$ shows that the Regge prediction works well for small $x \lesssim 10^{-2}$ and small $Q^{2} \lesssim 0.56 \mathrm{GeV}^{2}$, while pQCD takes over at $Q^{2} \gtrsim 1.5 \mathrm{GeV}^{2}$ in the measured $x$ range down to $x \gtrsim 3 \cdot 10^{-5}$. Thus we assume the simple Regge behavior of polarized partonic distributions,

$$
\Delta p\left(x, Q^{2}\right) \sim x^{\alpha}
$$

at $x \sim 0$ and low $Q_{\circ}^{2}$. 


\subsubsection{Shape of distributions}

Based on our previous considerations and following the ansatz of ref. [131] we parametrize the polarized partonic distributions at low $Q_{\circ}^{2}=0.34 \mathrm{GeV}^{2}$ as

$$
\Delta p\left(x, Q_{\circ}^{2}\right)=A_{p} x^{\alpha_{p}}(1-x)^{\beta_{p}} p\left(x, Q_{\circ}^{2}\right)
$$

where $p\left(x, Q_{\circ}^{2}\right)$ is the unpolarized partonic distribution, $p=u_{V}, d_{V}, \bar{q}$ or $G$, and $A_{p}, \alpha_{p}, \beta_{p}$ are parameters. Note that such a parametrization respects the Regge behavior, eq. (4.39), at low $x \sim 0$ and the power behavior, eq. (4.16), at high $x \sim 1$. The parameters are further constrained by the positivity requirement,

$$
|\Delta p(x)| \leq p(x)
$$

for all $x$ at the initial scale $Q_{o}^{2}$, leading to $\alpha_{p} \geq 0, \beta_{p} \geq 0$ and $A_{p} \leq 1$. If this requirement is satisfied at $Q_{0}^{2}$, the evolution assures that it holds for all $Q^{2} \geq Q_{\circ}^{2}$. Furthermore, the helicity retention property of eq. (4.18) implies $\beta_{p}=0$. We checked that the last assumption is consistent with the fits. That gives us the following initial distributions,

$$
\Delta p\left(x, Q_{\circ}^{2}\right)=A_{p} x^{\alpha_{p}} p\left(x, Q_{\circ}^{2}\right)
$$

in terms of eight $A_{p}, \alpha_{p}$ parameters. Note that the measured from byron beta decays results are not used to constrain $\Delta q_{3}$ of $\Delta q_{8}$ (see eq. (1.118) and (1.124), page 27). 
The unpolarized distributions $p\left(x, Q_{\circ}^{2}\right)$ are taken from ref. [149]. In the NLO they have the form:

$$
\begin{aligned}
u_{V}\left(x, Q_{\circ}^{2}\right) & =0.988 x^{-0.457}\left(1+1.58 \sqrt{x}+2.58 x+18.1 x^{3 / 2}\right)(1-x)^{3.380} \\
d_{V}\left(x, Q_{\circ}^{2}\right) & =0.182 x^{-0.684}\left(1+2.51 \sqrt{x}+25.0 x+11.4 x^{3 / 2}\right)(1-x)^{4.113} \\
\bar{q}\left(x, Q_{\circ}^{2}\right) & =0.545 x^{-0.70}(1+2.65 x)(1-x)^{8.33} \\
G\left(x, Q_{\circ}^{2}\right) & =26.20 x^{0.9}(1-x)^{4.0} .
\end{aligned}
$$

The inclusive deep inelastic scattering does not provide sufficient information about flavor separation of the sea. We thus assume that the light sea is initially symmetric, $\Delta \bar{u}\left(x, Q_{\circ}^{2}\right)=\Delta \bar{d}\left(x, Q_{\circ}^{2}\right) \equiv \Delta \bar{q}\left(x, Q_{\circ}^{2}\right)$. Furthermore we assume that the $x$ dependence of the polarized strange sea is the same, so that

$$
\Delta \bar{s}\left(x, Q_{\circ}^{2}\right)=\lambda_{s} \frac{\Delta \bar{u}+\Delta \bar{d}}{2}
$$

with the $S U(3)_{f}$ symmetry breaking parameter $\lambda_{s}$ varying between 1 and 0 . The choice $\lambda_{s}=1$ corresponds to the $S U(3)_{f}$ symmetric sea and $\lambda_{s}=0$ to the initially unpolarized strange sea.

\subsection{Fitting procedure}

The polarized distributions, expressed in terms of eight parameters at the initial $Q_{\circ}^{2}$ scale, can be perturbatively evolved to the appropriate values of $x$ and $Q^{2}$ at which the structure functions have been measured. Then the $g_{1}^{p}$ and $g_{1}^{n}$ structure functions are computed via convolution with the coefficient functions, eq. (4.3). The structure functions are related to each 
other via isospin interchange $u \leftrightarrow d$. The deuteron structure function $g_{1}^{d}$ is given by $g_{1}^{d}=(1 / 2)\left(g_{1}^{p}+g_{1}^{n}\right)\left(1-(3 / 2) \omega_{D}\right)$, with the $D$-state probability $\omega_{D}=0.05 \pm 0.01[150]$. The sum over data points of the square of the difference between the computed and measured structure function weighted by the statistical error of the measurement constitutes the $\chi^{2}$ of the fit. Minimizing it gives the fit parameters of the initial distributions.

We take into account all existing experimental results from CERN, SLAC and DESY for the $g_{1}$ of the proton $[16,18,20,60]$, neutron $[17,151,152]$ and deuteron $[19,21,60]$ with $Q^{2} \geq 1 \mathrm{GeV}^{2}$. If $g_{1}$ is not quoted directly, we determine the structure function at the measured $Q^{2}$ value from the $g_{1} / F_{1}$ result. The unpolarized structure function $F_{1}\left(x, Q^{2}\right)$ is obtained from the NMC [124] parametrization of $F_{2}\left(x, Q^{2}\right)$ and the fit to the SLAC [24, 25] data on the longitudinal to transverse photo-absorption cross sections, $R\left(x, Q^{2}\right)$. The kinematic range of the data is shown in fig. (4.3). We use the conventional fixed-flavor scheme $[131,149]$ and set $N_{f}=3$ in the Wilson coefficients and anomalous dimensions. The experimental data are at relatively low $Q^{2}$, and although above the pair-production threshold the heavier quark (charm and bottom) contribution to $g_{1}$ is negligible. The heavy quarks are included in the two-loop running coupling constant

$$
\frac{\alpha_{s}\left(Q^{2}\right)}{4 \pi}=\frac{1}{\beta_{\circ} \log \left(Q^{2} / \Lambda_{(f)}^{2}\right)}-\frac{\beta_{1}}{\beta_{\circ}^{3}} \frac{\log \left(\log \left(Q^{2} / \Lambda_{(f)}^{2}\right)\right)}{\left(\log \left(Q^{2} / \Lambda_{(f)}^{2}\right)\right)^{2}},
$$

where the coefficients of the $\beta$ function are $\beta_{\circ}=11-2 f / 3$ and $\beta_{1}=102-$ $38 \mathrm{f} / 3$. The number of active flavors $f$ in the coupling $\alpha_{s}\left(Q^{2}\right)$ equals the number of quarks with mass $m_{q}^{2} \leq Q^{2}$. For consistency with the fit to 


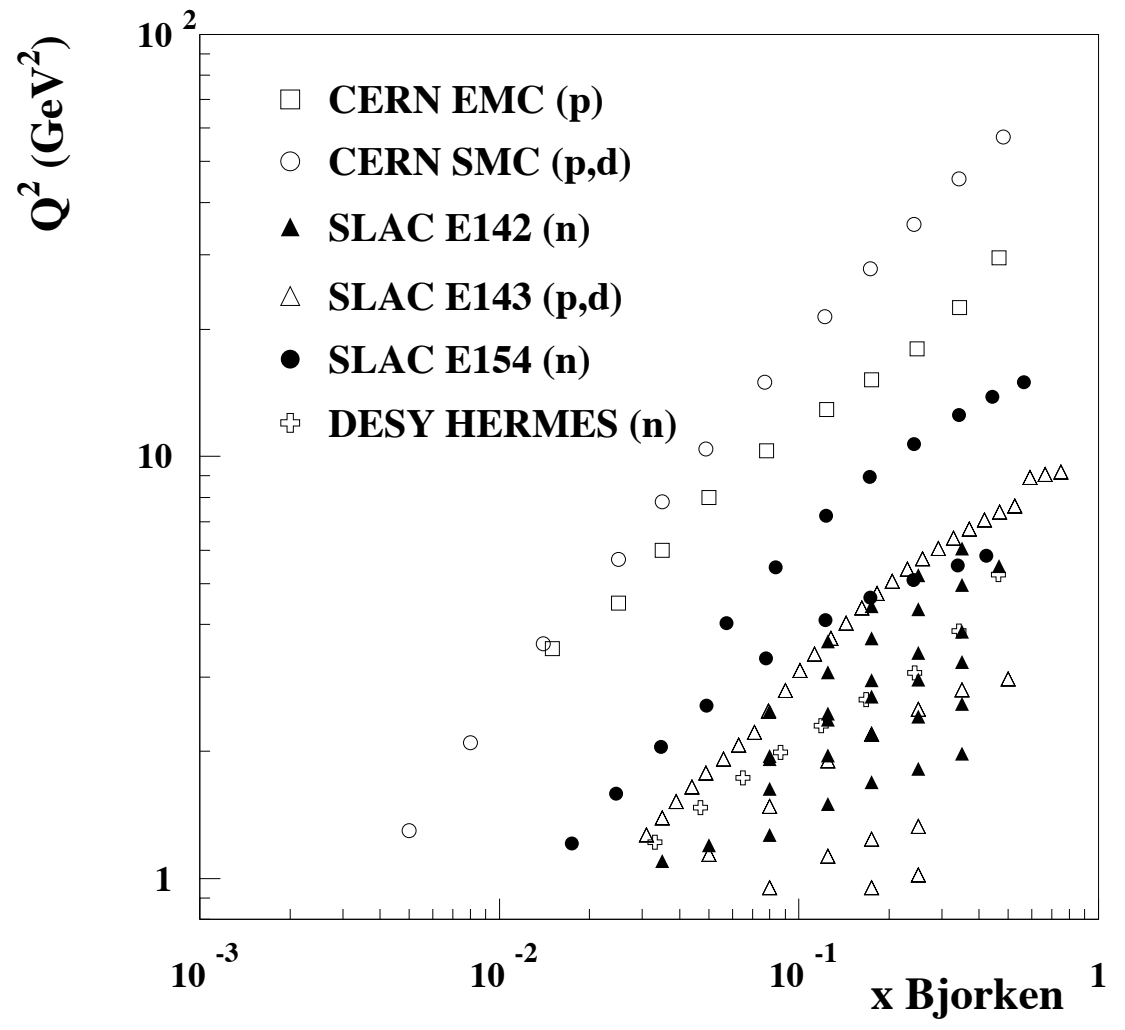

Figure 4.3: Kinematics coverage of the polarized DIS data.

the unpolarized distributions [149] we take $m_{c}=1.5 \mathrm{GeV}, m_{b}=4.5 \mathrm{GeV}$ and $\alpha_{s}\left(M_{Z}^{2}\right)=0.109$ or $\alpha_{s}\left(5 \mathrm{GeV}^{2}\right)=0.237$. The renormalization scales $\Lambda_{(f)}$ are determined by the continuity of $\alpha_{s}$ at the quark mass threshold, $\alpha_{s}\left(m_{q}^{2}, f\right)=\alpha_{s}\left(m_{q}^{2}, f+1\right)$, which gives

$$
\Lambda_{(3,4,5)}^{\overline{\mathrm{MS}}}=248,200,131 \mathrm{MeV} .
$$




\subsection{Mellin transform technique}

The evolution equations, see eq.(4.13), are integro-differential equations. To evolve the initial partonic distributions to a given $\left(x_{e x p}, Q_{e x p}^{2}\right)$ point, one needs to step through many intermediate $Q^{2}$ s (differential equations in $Q^{2}$ ) and at every step compute the distributions at all $x$ 's above $x_{\exp }$ (integral equations in $x$ ). This procedure is time consuming and susceptible to accumulative error. There is however a more direct way of evolution performed in the n-moment Mellin space. The nth moment $f(n)$ of the function $f(x)$ is defined via the Mellin transform,

$$
f(n)=\int_{0}^{1} d x x^{n-1} f(x)
$$

The useful feature of this transform is that it converts the integral convolution equation

$$
g_{1}(x) \equiv(C \otimes q)(x)=\int_{x}^{1} \frac{d y}{y} C\left(\frac{x}{y}\right) q(y),
$$

to a simple product

$$
g_{1}(n)=C(n) q(n)
$$

When applied to the evolution eq. (4.13), it gives a linear differential equation,

$$
\frac{d q(n, t)}{d t}=\frac{\alpha_{s}(t)}{2 \pi} P(n) q(n, t)
$$

that can be solved analytically $\left(\right.$ remember $\left.\alpha_{s}(t)=\frac{4 \pi}{\beta_{0} t}\right)$,

$$
q(n, t)=\left(\frac{\alpha_{s}(t)}{\alpha_{s}\left(t_{0}\right)}\right)^{\frac{-2 P(n)}{\beta_{0}}} q\left(n, t_{0}\right) .
$$


These directly evolved moments of the distributions $q(n, t)$ are easily "convoluted" with the moments $C(n)$ of the coefficient functions via eq. (4.49) to obtain the moments of the structure function, $g_{1}(n)$. We apply the inverse Mellin transform [153] in the complex $n$-plane to find the $x$ dependence,

$$
g_{1}(x)=\frac{1}{2 \pi i} \int_{c-i \infty}^{c+i \infty} d n x^{-n} g_{1}(n),
$$

where the real number $c$ is chosen so that the integral $\int_{c-i \infty}^{c+i \infty} d n x^{-n} g_{1}(n)$ is absolutely convergent, i.e. $c$ has to lie to the right of all singularities of $g_{1}(n)$

The evolution in the Mellin space is governed by the moments of the splitting functions $P(n)$, which are simply related to anomalous dimensions $\gamma(n)$. We follow the convention of ref. [154] for the anomalous dimensions

$$
\gamma(n)=\left(\frac{\alpha_{s}}{4 \pi}\right) \gamma^{(0)}(n)+\left(\frac{\alpha_{s}}{4 \pi}\right)^{2} \gamma^{(1)}(n)
$$

with

$$
\gamma^{(0)}(n)=-4 P^{(0)}(n) \quad \text { and } \quad \gamma^{(1)}(n)=-8 P^{(1)}(n)
$$

In the leading order [155, 138]

$$
\begin{aligned}
\gamma_{N S}^{\eta(0)}(n) & =\gamma_{q q}^{(0)}(n), \quad \eta= \pm 1, \\
\gamma_{q q}^{(0)}(n) & =4 C_{F}\left[2 S_{1}(n)-\frac{1}{n(n+1)}-\frac{3}{2}\right], \\
\gamma_{q G}^{(0)}(n) & =-8 T_{f} \frac{n-1}{n(n+1)}, \\
\gamma_{G q}^{(0)}(n) & =-4 C_{F} \frac{n+2}{n(n+1)}, \\
\gamma_{g G}^{(0)}(n) & =4 C_{A}\left[2 S_{1}(n)-\frac{4}{n(n+1)}-\frac{11}{6}\right]+\frac{8}{3} T_{f},
\end{aligned}
$$


where for the 3 flavors $C_{F}=4 / 3, C_{A}=3$ and $T_{f}=3 / 2$, and $S_{1}(n)$ is the analytical continuation of $\sum_{j=1}^{n} \frac{1}{j}$. Note that the first moments, $n=1$, of $\gamma_{q q}^{(0)}$, $\gamma_{q G}^{(0)}$ and $\gamma_{N S}^{\eta(0)}$ vanish as a consequence of helicity conservation at the quarkgluon vertex. Thus the first moments $\Delta q_{N S}^{\eta}, \Delta \Sigma$ of the quark distributions (see eq. (4.5-4.6)) are conserved (i.e. $Q^{2}$ independent) in leading order. The NLO formulas in the $\overline{\mathrm{MS}}$ factorization scheme are given in the Appendix B.2 (page 279).

\subsection{Dependence on the renormalization and factorization schemes}

In expressing physical quantities as a series in the strong coupling constant $\alpha_{s}$ we have to sum over all the terms in order for the result to be independent of the renormalization scheme. If the expansion is truncated, we introduce renormalization scheme dependence into the approximation. This dependence has two sources. The first is the the regularization prescription or procedure: what finite pieces are subtracted together with the divergent pieces. That accounts, e.g., for the ambiguity in the expansion coefficients of the function $\beta\left(\alpha_{s}\right)$ in the renormalization group equation for the running of the strong coupling constant $\alpha_{s}$. The first two coefficients in the $\beta$ function expansion are the same [156] for any regularization prescription. Thus there is no ambiguity in the leading and next-to-leading order. The second source is the freedom in specification of the boundary condition for the solution to the renormalization group equation for $\alpha_{s}(\mu)$, where $\mu$ is the renormaliza- 
tion scale. It is usually specified by introducing the renormalization scale parameter $\Lambda_{Q C D}$. There are several prescriptions [157] for setting its value. One estimates the uncertainty associated with the choice by varying $\Lambda_{Q C D}$. The solution for $\alpha_{s}(\mu)$ depends on $\mu$ and $\Lambda_{Q C D}$ only via the ratio $\mu / \Lambda_{Q C D}$. Equivalently one can fix the value of $\Lambda_{Q C D}$ and regard the renormalization as $\mu$ dependent. To summarize: the renormalization scheme is defined by specification of both the renormalization procedure and the renormalization scale. In the NLO only the latter produces an ambiguity. This introduces an uncertainty in the fits.

A similar situation arises during the factorization, theorem (4.1), of the structure functions into partonic distributions and hard scattering crosssections, theorem (4.1). The factorization can be viewed as the renormalization of a composite operator in addition to and independent of the coupling constant renormalization described above. Again there is a dependence on the renormalization procedure which we call factorization scheme dependence. As mentioned in section 4.1 it is already important in NLO. We also have a factorization scale dependence reflecting the freedom in the choice of the renormalization scale for the considered composite operator. In the polarized case there is a further complication due to the ambiguity in the extension of $\gamma_{5}$ and the Levi-Civita $\epsilon_{i j k l}$ to $n \neq 4$ dimensions $[158,159]$ in the dimensional regularization. We absorb it in the definition of the factorization procedure. Thus, the factorization scheme is uniquely defined by choosing the factorization procedure and scale. For brevity one often refers 
to a certain factorization scheme, meaning that both the renormalization and factorization schemes are defined.

Given a set of coefficient functions and anomalous dimensions in one factorization scheme, a transformation to another scheme [160] can be constructed. Let us start with eq. (4.49) for the moments of the structure function (we suppress the moment label $\mathrm{n}$ and show the tependence explicitly),

$$
g_{1}(t)=C(t) q(t)
$$

where $q(t)$ satisfies the evolution eq. (4.50),

$$
\frac{d q(t)}{d t}=\frac{\alpha_{s}(t)}{2 \pi} P(t) q(t)
$$

The Wilson coefficients in a new scheme $\hat{C}(t)$ are related to the old coefficients via finite renormalization

$$
\hat{C}(t)=C(t) Z^{-1}(t)
$$

where

$$
\begin{aligned}
& Z(t)=1+\frac{\alpha_{s}(t)}{2 \pi} Z^{(1)}, \\
& C(t)=C^{(0)}+\frac{\alpha_{s}(t)}{2 \pi} C^{(1)} .
\end{aligned}
$$

That immediately gives us the transformation

$$
\hat{C}^{(1)}=C^{(1)}-C^{(0)} Z^{(1)}
$$

of the coefficient functions from one scheme to another. For $g_{1}(t)$ to be scheme independent, $g_{1}=C q=\hat{C} \hat{q}$, the distributions $q(t)$ are redefined,

$$
\hat{q}(t)=Z(t) q(t)
$$


and the evolution equation transforms to

$$
\frac{d \hat{q}(t)}{d t}=\frac{\alpha_{s}(t)}{2 \pi} \hat{P}(t) \hat{q}(t)
$$

Replacing $\hat{q}(t)$ by the product $Z(t) q(t)$ on the left hand side, we make use of the evolution eq. (4.57) and $\frac{d \alpha_{s}}{d t}=-\frac{\beta_{0}}{4 \pi} \alpha_{s}^{2}\left(\right.$ so $\left.\frac{d Z(t)}{d t}=-\frac{\beta_{0}}{8 \pi^{2}} \alpha_{s}^{2} Z^{(1)}\right)$ and obtain

$$
\hat{P}=Z P Z^{-1}-\frac{\beta_{\circ} \alpha_{s}}{4 \pi} Z^{(1)} Z
$$

Thus

$$
\hat{P}^{(1)}=P^{(1)}+\left[Z^{(1)}, P^{(0)}\right]-\frac{\beta_{\circ}}{2} Z^{(1)},
$$

or equivalently in terms of the anomalous dimensions, see eq. (4.54),

$$
\hat{\gamma}^{(1)}=\gamma^{(1)}+2\left[Z^{(1)}, \gamma^{(0)}\right]+4 \beta_{\circ} Z^{(1)}
$$

Collecting the results for the polarized case $\left(C_{q}^{(0)}(n)=1, C_{G}^{(0)}(n)=0\right)$, we write the transformation between factorization schemes as

$$
\begin{aligned}
\gamma_{N S}^{\eta(1)}(n) & \rightarrow \gamma_{N S}^{\eta(1)}(n)+4 \beta \circ Z_{q q}^{(1)}(n) \\
\gamma_{i j}^{(1)}(n) & \rightarrow \gamma_{i j}^{(1)}(n)+2\left[Z^{(1)}(n), \gamma^{(0)}\right]_{i j}+4 \beta \circ Z_{i j}^{(1)}(n) \\
C_{q}^{(1)}(n) & \rightarrow C_{q}^{(1)}(n)-Z_{q q}^{(1)}(n) \\
C_{G}^{(1)}(n) & \rightarrow C_{G}^{(1)}(n)-Z_{q G}^{(1)}(n)
\end{aligned}
$$

where $Z^{(1)}(n)$ is an arbitrary $2 \times 2$ transformation matrix, and $i, j \in\{q, G\}$. The $\overline{\mathrm{MS}}$ scheme with the t'Hooft and Veltman [159] $\gamma_{5}$ prescription were used for the recently calculated [130] NLO anomalous dimensions and Wilson coefficients. In this scheme the first moment of the gluon coefficient function 
vanishes, $C_{G}^{(1)}(n=1)=0$, so that the gluon distribution does not contribute to the integral of $g_{1}$. There is a different scheme, the Adler-Bargeen or $A B$ $[38,158,161]$ scheme, in which the integral of $g_{1}$ includes the axial anomaly contribution, $\left(-\alpha_{s} / 4 \pi\right) \sum_{q} e_{q}^{2} \Delta G$, leading to

$$
C_{G}^{(1)}(n=1)=-N_{f}
$$

The extra contribution can be absorbed into the redefined total quark helicity,

$$
\Delta \Sigma_{A B}=\Delta \Sigma_{\overline{\mathrm{MS}}}\left(Q^{2}\right)+N_{f} \frac{\alpha_{s}\left(Q^{2}\right)}{2 \pi} \Delta G\left(Q^{2}\right)
$$

making it a conserved, i.e. $Q^{2}$ independent, quantity to all orders [162]. In the leading order $\Delta \Sigma_{\overline{\mathrm{MS}}}$ also is conserved, making the product $\alpha_{s}\left(Q^{2}\right) \Delta G\left(Q^{2}\right)$ independent of $Q^{2}$ [163]. That gives a logarithmically divergent in $Q^{2}$, $1 / \alpha_{s}\left(Q^{2}\right)$ behavior of $\Delta G\left(Q^{2}\right)$ compensated by the appropriate change of the orbital angular momentum $[37,164] L_{z}$ in order to preserve the nucleon spin $\frac{1}{2}=\frac{1}{2} \Delta \Sigma+\Delta G+L_{z}$ (cf. section 1.7.1, page 23). It further implies that the ambiguity in the definition of the nucleon helicity content $\Delta \Sigma$ persists at any finite and even infinite $Q^{2}$. It also indicates that the factorization scheme has to be explicitly specified for a meaningful interpretation of the results.

A transformation from the $\overline{\mathrm{MS}}$ to $A B$ scheme was constructed in ref. [132]. The gluon coefficient function in terms of eq. (4.68) implies $Z_{q G}^{(1)}(n=1)$ to be proportional to $N_{f}$. The conservation of the non-singlet axial current requires $Z_{q q}^{(1)}(n=1)=0$. Choosing the lower entries to the $Z^{(1)}$ transformation matrix to vanish and then requiring that $Z^{(1)}(x)$, the inverse Mellin transform 
of $Z^{(1)}(n)$, be $x$ independent gives

$$
Z^{(1)}(n)_{\overline{\mathrm{MS}} \rightarrow A B}=\frac{1}{n}\left(\begin{array}{cc}
0 & N_{f} \\
0 & 0
\end{array}\right) .
$$

This transformation minimally modifies the $\overline{\mathrm{MS}}$ scheme in the sense that it preserves the low and high $x$ asymptotic behavior of the coefficient functions and anomalous dimensions and thus of the partonic distributions. We perform our fits in both schemes in order to demonstrate the factorization scheme dependence of the results.

\subsection{Analysis of errors}

The multi-parameter fit was performed using the MINUIT routine [165] from the CERN Program Library. The points are weighted by statistical errors. The routine returns the correlation matrix so the statistical errors on the values of fitted parameters can, in principle, be computed. The estimates of MINUIT should however be taken with some caution. The $\chi^{2}$ distribution around the minimum in the parameter space is quite shallow, usually non-symmetric, and the returned correlation matrix is not always accurate. Furthermore, it is difficult to incorporate the correlated systematic errors

or theoretical uncertainties into the $\chi^{2}$ formalism. Instead of relying on the MINUIT estimates we propagate the errors in the standard way.

\subsubsection{Statistical errors}

The statistical errors in the fit parameters and in the first moments of the distributions $\Delta q, \Delta G$ and structure functions $\Gamma_{1}^{p}, \Gamma_{1}^{n}$ etc. were computed 
as follows: Each experimental point was varied within its statistical error in accordance with a Gaussian distribution, with the mean value corresponding

to the measurement $g_{1}^{i}$ and the variance equal to the error $\sigma_{\text {stat }}^{2}\left(g_{1}^{i}\right)$. After all points are randomized independently and simultaneously the NLO fit is repeated. A typical sample consisted of 800 fits. The distributions of the first moments of the partonic densities are shown in fig. 4.7.1. The distributions are neither Gaussian nor symmetric. The mean and the RMS are not good measures of the result and its uncertainty. We take the most probable value, say $f$, as the result. The left $\sigma_{-}$and right $\sigma_{+}$errors are defined so that there is a $34 \%$ probability for the result $f$ to be within the interval $\left(f-\sigma_{-}\right)$and a $34 \%$ probability for it to be within $\left(f+\sigma_{+}\right)$. This method for statistical error propagation corresponds to adding the statistical errors of each measurement in quadrature while properly accounting for nonlinearities.

\subsubsection{Systematic errors}

The systematic errors of the measurements are usually dominated by normalization errors in the target, beam polarization, dilution factor etc. To a large extent they are point to point correlated within one experiment. We treat them as fully correlated for a given experiment. Thus we generate independently a Gaussian distributed random variable for each experiment that represents a fraction of the systematic error by which all the points from the experiment are simultaneously shifted. Again we repeat the fit after all the points are randomized in a fully correlated manner within each experiment while uncorrelated between experiments. This procedure adds 

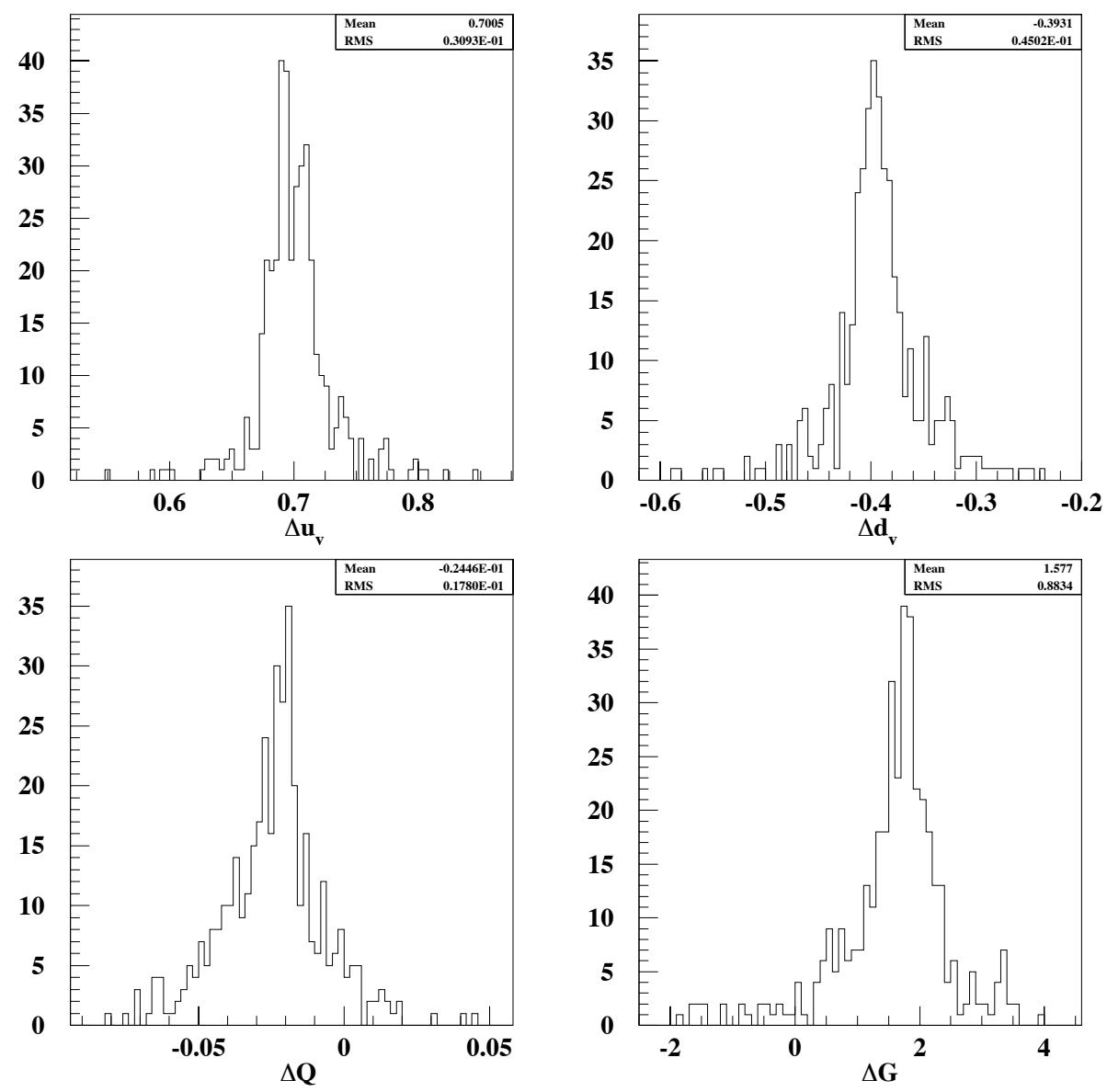

Figure 4.4: Distributions of the first moments of the parton densities in the $\overline{\mathrm{MS}}$ scheme due to the 'statistical' randomization of the $g_{1}$ data, as described in the text.

the contributions from systematic errors linearly within each experiment, and then adds the contributions from all the experiments quadratically, assuming the systematics of the independent experiments are uncorrelated. 


\subsubsection{Theoretical uncertainties}

The biggest source of the theoretical uncertainty is the error in the value of $\alpha_{s}$. This error contains the renormalization and factorization scale dependencies. We estimate it by repeating the fits with $\alpha_{s}\left(M_{Z}^{2}\right)$ varied in the range allowed by the fixed target unpolarized DIS experiments [71], i.e. $\alpha_{s}\left(M_{Z}^{2}\right)=0.108-0.116$. We also vary the current quark masses in the range $m_{c}=1-2 \mathrm{GeV}$ and $m_{b}=4-5 \mathrm{GeV}$, which affects the running of $\alpha_{s}$. The effect of $\mathrm{SU}(3)_{\text {flavor }}$ breaking is estimated by varying the parameter $\lambda_{s}$ from

1 to 0 . We also vary the D-state correction $\left(\omega_{D}\right)$ to $g_{1}^{d}$ within its uncertainty. Those factors are found to have a small influence on the results. To test the sensitivity to the $x$ dependence of the initial distributions and the value of the starting scale $Q_{\circ}^{2}$, we repeat the fit with initial unpolarized distributions taken from ref. [166] at $Q_{\circ}^{2}=1 \mathrm{GeV}^{2}$ and find results consistent with our values within the quoted statistical uncertainties. Possible higher twist effects are neglected. They are expected to drop at high $x$ as $1 / W^{2}$ [167], and the cut $W^{2}>4 \mathrm{GeV}^{2}$ has been applied to all the data with the majority of them exceeding $W^{2}>8 \mathrm{GeV}^{2}$. At low $x$ we have an indication from the unpolarized data [127] that the leading twist-two perturbative description is valid for $Q^{2}$ above $0.5 \mathrm{GeV}^{2}$ down to $x \sim 10^{-5}$.

\subsection{Results}

The best fit coefficients are listed in table 4.1 [152]. The $\chi^{2}$ of the fit to the total of 168 points is 146 in the $\overline{\mathrm{MS}}$ scheme and 148 in the $A B$ scheme. 
Table 4.1: Fitted Values of the Free Parameters in eq. (4.42) in the $\overline{\mathrm{MS}}$ and AB Schemes. Also Quoted are the Statistical, Systematic, and Theoretical Errors.

\begin{tabular}{|c|c|c|c|c|c|c|c|c|}
\hline & \multicolumn{4}{|c|}{$\overline{\mathrm{MS}}$} & \multicolumn{4}{|c|}{$\mathrm{AB}$} \\
\hline & Value & Stat. & Syst. & Theory & Value & Stat. & Syst. & Theory \\
\hline$A_{u}$ & 0.99 & $\begin{array}{l}+0.08 \\
-0.08\end{array}$ & $\begin{array}{l}+0.04 \\
-0.05\end{array}$ & $\begin{array}{l}+0.97 \\
-0.11\end{array}$ & 0.98 & $\begin{array}{l}+0.07 \\
-0.06\end{array}$ & $\begin{array}{l}+0.05 \\
-0.07\end{array}$ & $\begin{array}{l}+0.96 \\
-0.09\end{array}$ \\
\hline$A_{d}$ & -0.78 & $\begin{array}{l}+0.14 \\
-0.20\end{array}$ & $\begin{array}{l}+0.05 \\
-0.05\end{array}$ & $\begin{array}{l}+0.05 \\
-1.28\end{array}$ & -0.82 & $\begin{array}{l}+0.06 \\
-0.11\end{array}$ & $\begin{array}{l}+0.07 \\
-0.06\end{array}$ & $\begin{array}{l}+0.31 \\
-1.21\end{array}$ \\
\hline$A_{Q}$ & -0.02 & $\begin{array}{l}+0.03 \\
-0.06\end{array}$ & $\begin{array}{l}+0.01 \\
-0.02\end{array}$ & $\begin{array}{l}+0.01 \\
-0.35\end{array}$ & -0.04 & $\begin{array}{l}+0.02 \\
-0.05\end{array}$ & $\begin{array}{l}+0.01 \\
-0.02\end{array}$ & $\begin{array}{l}+0.03 \\
-0.06\end{array}$ \\
\hline$A_{G}$ & 1.6 & $\begin{array}{l}+1.1 \\
-0.9\end{array}$ & $\begin{array}{l}+0.6 \\
-0.6\end{array}$ & $\begin{array}{l}+0.2 \\
-1.3\end{array}$ & 0.1 & $\begin{array}{l}+1.0 \\
-0.3\end{array}$ & $\begin{array}{l}+0.5 \\
-0.2\end{array}$ & $\begin{array}{l}+0.1 \\
-0.6\end{array}$ \\
\hline$\alpha_{u}$ & 0.63 & $\begin{array}{l}+0.06 \\
-0.07\end{array}$ & $\begin{array}{l}+0.04 \\
-0.05\end{array}$ & $\begin{array}{l}+0.36 \\
-0.06\end{array}$ & 0.55 & $\begin{array}{l}+0.08 \\
-0.06\end{array}$ & $\begin{array}{l}+0.03 \\
-0.04\end{array}$ & $\begin{array}{l}+0.56 \\
-0.05\end{array}$ \\
\hline$\alpha_{d}$ & 0.28 & $\begin{array}{l}+0.15 \\
-0.11\end{array}$ & $\begin{array}{l}+0.05 \\
-0.03\end{array}$ & $\begin{array}{l}+0.75 \\
-0.03\end{array}$ & 0.40 & $\begin{array}{l}+0.20 \\
-0.12\end{array}$ & $\begin{array}{l}+0.07 \\
-0.13\end{array}$ & $\begin{array}{l}+0.53 \\
-0.34\end{array}$ \\
\hline$\alpha_{Q}$ & 0.04 & $\begin{array}{l}+0.29 \\
-0.03\end{array}$ & $\begin{array}{l}+0.12 \\
-0.03\end{array}$ & $\begin{array}{l}+0.55 \\
-0.01\end{array}$ & 0.00 & $\begin{array}{l}+0.17 \\
-0.00\end{array}$ & $\begin{array}{l}+0.17 \\
-0.00\end{array}$ & $\begin{array}{l}+0.00 \\
-0.00\end{array}$ \\
\hline$\alpha_{G}$ & 0.8 & $\begin{array}{l}+0.4 \\
-0.5 \\
\end{array}$ & $\begin{array}{l}+0.3 \\
-0.3 \\
\end{array}$ & $\begin{array}{l}+0.1 \\
-0.6 \\
\end{array}$ & 0.0 & $\begin{array}{l}+0.7 \\
-0.0 \\
\end{array}$ & $\begin{array}{l}+1.0 \\
-0.0 \\
\end{array}$ & $\begin{array}{l}+1.0 \\
-0.0 \\
\end{array}$ \\
\hline
\end{tabular}

The results for the structure functions of the proton, $g_{1}^{p}$, and neutron, $g_{1}^{n}$, at $5 \mathrm{GeV}^{2}$ are compared with the experimental data in fig. 4.5. Our analysis agrees with $[131,132,133,134]$ in predicting that the proton structure function crosses zero between $x=0.001$ and $x=0.01$ (at $Q^{2}=5 \mathrm{GeV}^{2}$ ). This is due to the sea and gluon contributions that start to dominate at sufficiently low $x$. The effect of the sea and gluon contributions is even more prominent in the deuteron structure function $g_{1}^{d}$, which is expected to cross zero near $x=0.01$. 

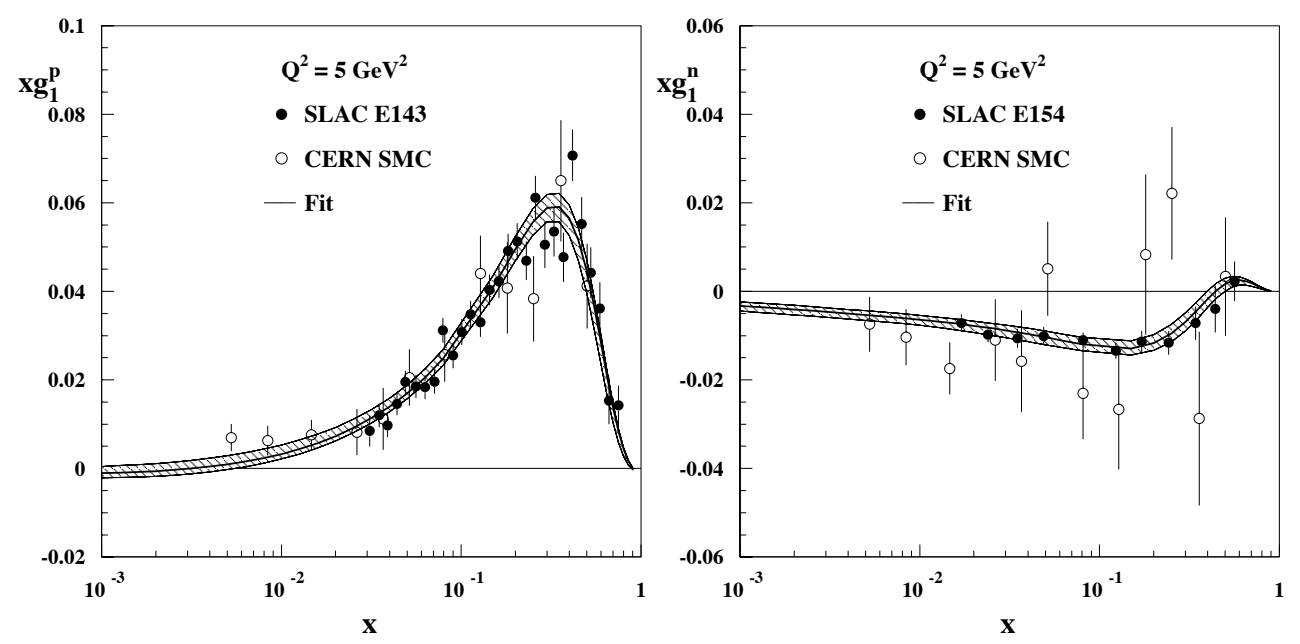

Figure 4.5: The structure functions $x g_{1}^{p}$ and $x g_{1}^{n}$ at $Q^{2}=5 \mathrm{GeV}^{2}$. E143, SMC, and E154 data have been evolved to $Q^{2}=5 \mathrm{GeV}^{2}$ using a procedure described in the text. The result of the $\overline{\mathrm{MS}}$ fit is shown by the solid line. The hatched area represents the total error of the fit.

The first moments of the parton distributions and the first moments of the structure functions at $Q^{2}=5 \mathrm{GeV}^{2}$ are given in table 4.2. We observe that the first moments of the valence quark distributions are determined fairly well, but that the moments of the sea quarks and gluons are only qualitatively constrained. There is an apparent $\approx 2 \sigma$ disagreement of $\Delta q_{3}$ with the value extracted from the neutron beta decay $\Delta q_{3}=g_{A}=1.2601 \pm 0.0025$ [71]. This is because the calculation is done in NLO and the higher order corrections to the Bjorken sum rule are not taken into account. The corrections can be as large as $5 \%$ [48] at the weighted world average $Q^{2} \approx 5 \mathrm{GeV}^{2}$, and they bring $\Delta q_{3}$ in better agreement with the beta decay data. For consistency with the NLO approximation, we do not include this correction, which has no effect on 
Table 4.2: First Moments of the Polarized Parton Distributions and Structure Functions of the Proton, Neutron, and Deuteron in the $\overline{\mathrm{MS}}$ and $A B$ Schemes Evaluated at $Q^{2}=5 \mathrm{GeV}^{2}$. The Errors are Statistical, Systematic, and Theoretical.

\begin{tabular}{|c|c|c|c|c|c|c|c|c|}
\hline & \multicolumn{4}{|c|}{$\overline{\mathrm{MS}}$} & \multicolumn{4}{|c|}{$\mathrm{AB}$} \\
\hline & Value & Stat. & Syst. & Theory & Value & Stat. & Syst. & Theory \\
\hline$\Delta u_{V}$ & 0.69 & $\begin{array}{l}+0.03 \\
-0.02\end{array}$ & $\begin{array}{l}+0.05 \\
-0.04\end{array}$ & $\begin{array}{l}+0.14 \\
-0.01\end{array}$ & 0.74 & $\begin{array}{l}+0.03 \\
-0.02\end{array}$ & $\begin{array}{l}+0.03 \\
-0.03\end{array}$ & $\begin{array}{l}+0.07 \\
-0.01\end{array}$ \\
\hline$\Delta d_{V}$ & -0.40 & $\begin{array}{l}+0.03 \\
-0.04\end{array}$ & $\begin{array}{l}+0.03 \\
-0.03\end{array}$ & $\begin{array}{l}+0.07 \\
-0.00\end{array}$ & -0.33 & $\begin{array}{l}+0.03 \\
-0.04\end{array}$ & $\begin{array}{l}+0.03 \\
-0.05\end{array}$ & $\begin{array}{l}+0.01 \\
-0.03\end{array}$ \\
\hline$\Delta \bar{Q}$ & -0.02 & $\begin{array}{l}+0.01 \\
-0.02\end{array}$ & $\begin{array}{l}+0.01 \\
-0.01\end{array}$ & $\begin{array}{l}+0.00 \\
-0.03\end{array}$ & -0.03 & $\begin{array}{l}+0.02 \\
-0.02\end{array}$ & $\begin{array}{l}+0.01 \\
-0.01\end{array}$ & $\begin{array}{l}+0.01 \\
-0.01\end{array}$ \\
\hline$\Delta G$ & 1.8 & $\begin{array}{l}+0.6 \\
-0.7\end{array}$ & $\begin{array}{l}+0.4 \\
-0.5\end{array}$ & $\begin{array}{l}+0.1 \\
-0.6\end{array}$ & 0.4 & $\begin{array}{l}+1.0 \\
-0.7\end{array}$ & $\begin{array}{l}+0.9 \\
-0.6\end{array}$ & $\begin{array}{l}+1.1 \\
-0.1\end{array}$ \\
\hline$\Delta q_{3}$ & 1.09 & $\begin{array}{l}+0.03 \\
-0.02\end{array}$ & $\begin{array}{l}+0.05 \\
-0.05\end{array}$ & $\begin{array}{l}+0.06 \\
-0.01\end{array}$ & 1.07 & $\begin{array}{l}+0.03 \\
-0.02\end{array}$ & $\begin{array}{l}+0.06 \\
-0.06\end{array}$ & $\begin{array}{l}+0.10 \\
-0.01\end{array}$ \\
\hline$\Delta q_{8}$ & 0.30 & $\begin{array}{l}+0.06 \\
-0.05\end{array}$ & $\begin{array}{l}+0.05 \\
-0.05\end{array}$ & $\begin{array}{l}+0.23 \\
-0.01\end{array}$ & 0.42 & $\begin{array}{l}+0.05 \\
-0.08\end{array}$ & $\begin{array}{l}+0.06 \\
-0.06\end{array}$ & $\begin{array}{l}+0.03 \\
-0.01\end{array}$ \\
\hline$\Delta \Sigma$ & 0.20 & $\begin{array}{l}+0.05 \\
-0.06\end{array}$ & $\begin{array}{l}+0.04 \\
-0.05\end{array}$ & $\begin{array}{l}+0.01 \\
-0.01\end{array}$ & 0.25 & $\begin{array}{l}+0.07 \\
-0.07\end{array}$ & $\begin{array}{l}+0.05 \\
-0.05\end{array}$ & $\begin{array}{l}+0.05 \\
-0.02\end{array}$ \\
\hline$\Delta q_{0}$ & 0.20 & $\begin{array}{l}+0.05 \\
-0.06\end{array}$ & $\begin{array}{l}+0.04 \\
-0.05\end{array}$ & $\begin{array}{l}+0.01 \\
-0.01\end{array}$ & 0.21 & $\begin{array}{l}+0.05 \\
-0.06\end{array}$ & $\begin{array}{l}+0.06 \\
{ }_{-0.07}\end{array}$ & $\begin{array}{l}+0.05 \\
-0.02\end{array}$ \\
\hline$\Gamma_{1}^{p}$ & 0.112 & $\begin{array}{l}+0.006 \\
-0.006\end{array}$ & $\begin{array}{l}+0.008 \\
{ }_{-0.008}\end{array}$ & $\begin{array}{l}+0.009 \\
-0.001\end{array}$ & 0.114 & $\begin{array}{l}+0.005 \\
-0.006\end{array}$ & $\begin{array}{l}+0.010 \\
-0.011\end{array}$ & $\begin{array}{l}+0.001 \\
-0.003\end{array}$ \\
\hline$\Gamma_{1}^{n}$ & -0.056 & $\begin{array}{l}+0.006 \\
-0.007\end{array}$ & $\begin{array}{l}+0.005 \\
-0.006\end{array}$ & $\begin{array}{l}+0.002 \\
-0.001\end{array}$ & -0.051 & $\begin{array}{l}+0.005 \\
-0.006\end{array}$ & $\begin{array}{l}+0.006 \\
-0.007\end{array}$ & $\begin{array}{l}+0.001 \\
-0.012\end{array}$ \\
\hline$\Gamma_{1}^{d}$ & 0.026 & $\begin{array}{l}+0.005 \\
-0.006\end{array}$ & $\begin{array}{l}+0.005 \\
-0.006\end{array}$ & $\begin{array}{l}+0.005 \\
-0.001\end{array}$ & 0.029 & $\begin{array}{l}+0.004 \\
-0.005\end{array}$ & $\begin{array}{l}+0.007 \\
-0.008\end{array}$ & $\begin{array}{l}+0.001 \\
-0.007\end{array}$ \\
\hline$\Gamma_{1}^{p-n}$ & 0.168 & $\begin{array}{l}+0.005 \\
-0.004 \\
\end{array}$ & $\begin{array}{l}+0.008 \\
-0.007 \\
\end{array}$ & $\begin{array}{l}+0.007 \\
-0.001 \\
\end{array}$ & 0.165 & $\begin{array}{l}+0.004 \\
-0.004 \\
\end{array}$ & $\begin{array}{l}+0.009 \\
-0.009 \\
\end{array}$ & $\begin{array}{l}+0.013 \\
-0.001 \\
{ }_{-0}\end{array}$ \\
\hline
\end{tabular}

the physical observable $g_{1}$. The contribution of the experimental systematic errors to the first moments of the parton distributions is comparable with the statistical contribution. Therefore, the full error in the first moment of the gluon distribution $\Delta G$ is larger than quoted in ref. [132], even though the new 
data from E154 were added. The theoretical uncertainty is also quite large. It could potentially be reduced if the simultaneous analysis of the unpolarized and polarized data was performed (including $\alpha_{s}$ as one of the parameters). The results of the fits in both $\overline{\mathrm{MS}}$ and $A B$ schemes are consistent within errors. The fits are significantly less stable in the $A B$ scheme. Note that the values of the singlet axial charge $\Delta q_{0}$, which equals $\Delta \Sigma$ in the $\overline{\mathrm{MS}}$ scheme and $\Delta \Sigma-N_{f}\left(\alpha_{s} /(2 \pi)\right) \Delta G$ in the $A B$ scheme, are almost exactly the same in both schemes.

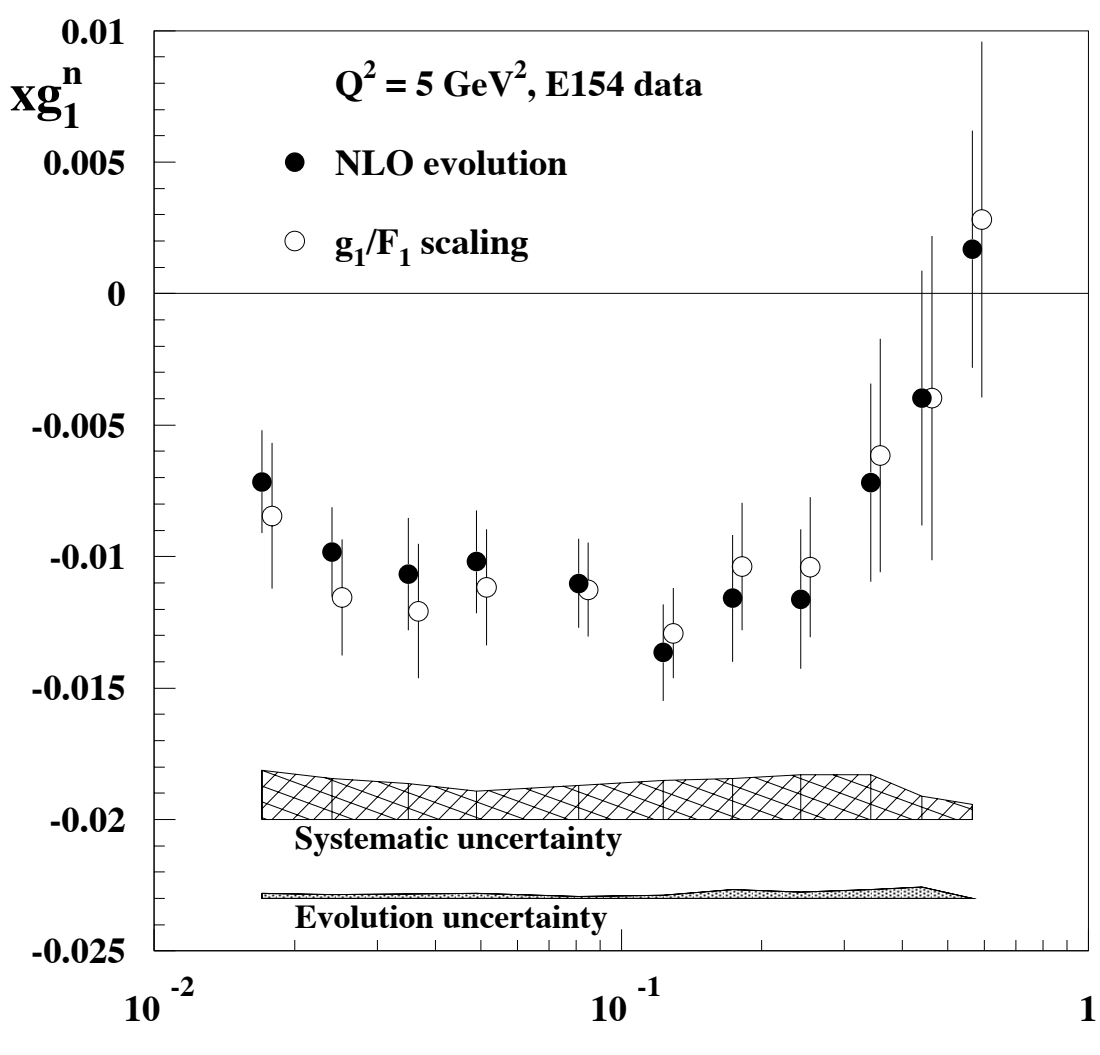

Figure 4.6: The structure function $x g_{1}^{n}$ evolved to $Q^{2}=5 \mathrm{GeV}^{2}$ using our $\overline{\mathrm{MS}}$ parametrization, and assuming that $g_{1}^{n} / F_{1}^{n}$ is independent of $Q^{2}$. 
Using the parametrization of the parton distributions, one can obtain the polarized structure function $g_{1}^{f i t}$ and evolve the experimental data points to a common $\left\langle Q^{2}\right\rangle$ using the formula:

$$
g_{1}^{e x p}\left(x_{i},\left\langle Q^{2}\right\rangle\right)=g_{1}^{e x p}\left(x_{i}, Q_{i}^{2}\right)-\delta g_{1}^{f i t}\left(x_{i}, Q_{i}^{2},\left\langle Q^{2}\right\rangle\right)
$$

with

$$
\delta g_{1}^{f i t}\left(x_{i}, Q_{i}^{2},\left\langle Q^{2}\right\rangle\right)=g_{1}^{f i t}\left(x_{i}, Q_{i}^{2}\right)-g_{1}^{f i t}\left(x_{i},\left\langle Q^{2}\right\rangle\right)
$$

where $g_{1}^{e x p}\left(x_{i}, Q_{i}^{2}\right)$ is the structure function measured at the experimental kinematics. The errors in $g_{1}^{\exp }\left(x_{i},\left\langle Q^{2}\right\rangle\right)$ have three sources:

$$
\sigma^{2}\left(g_{1}^{e x p}\left(x_{i}, Q_{i}^{2}\right)\right)=\sigma^{2}\left(g_{1}^{e x p}\right)_{s t a t}+\sigma^{2}\left(g_{1}^{e x p}\right)_{s y s t}+\sigma^{2}\left(g_{1}\right)_{e v o l},
$$

where statistical and systematic uncertainties take into account the correlation between $g_{1}^{e x p}\left(x_{i}, Q_{i}^{2}\right)$ and $g_{1}^{f i t}\left(x_{i}, Q_{i}^{2}\right)$, and the evolution uncertainty only includes uncorrelated experimental uncertainties added in quadrature. Table 4.3 lists the E154 data points evolved to the common $\left\langle Q^{2}\right\rangle=5 \mathrm{GeV}^{2}$ using this procedure. The NLO evolution is compared to the traditional scaling of $g_{1}^{n} / F_{1}^{n}$ in fig. 4.6. The difference is comparable with the precision of the present day experiments and cannot be neglected. The $Q^{2}$ dependence of the ratio $g_{1} / F_{1}$ is shown in fig. 4.7 . We plot the difference between the values of $g_{1} / F_{1}$ at a given $Q^{2}$ and $Q^{2}=5 \mathrm{GeV}^{2}$ to which the SLAC data are evolved. For the neutron the evolution of $g_{1}^{n}$ is slower than that of $F_{1}^{n}$. Therefore, assuming scaling of $g_{1}^{n} / F_{1}^{n}$, one typically overestimates the absolute value of $g_{1}^{n}\left(x,\left\langle Q^{2}\right\rangle\right)$ at low $x$ (where $Q_{i}^{2}<\left\langle Q^{2}\right\rangle$ ) and underestimates it at high $x$ (where $\left.Q_{i}^{2}\right\rangle\left\langle Q^{2}\right\rangle$ ). The two effects approximately cancel in the integral 
Table 4.3: E154 Results for $g_{1}^{n}$ Evolved to $\left\langle Q^{2}\right\rangle=5 \mathrm{GeV} 2$ Assuming $g_{1} / F_{1}$ is Independent of $Q^{2}$ and According to Eq. (4.71). The Errors Were Propagated as Described in the Text.

\begin{tabular}{|c|c|c|c|}
\hline$x_{i}$ & $\begin{array}{c}Q_{i}^{2} \\
\mathrm{GeV}^{2} \\
\end{array}$ & $\begin{aligned} & g_{1}^{n}\left(x_{i}, Q_{i}^{2}\right) \\
& \pm \text { stat. } \pm \text { syst. }\end{aligned}$ & $\begin{array}{c}g_{1}^{n}\left(x_{i}, 5 \mathrm{GeV}^{2}\right) \\
\pm \text { stat. } \pm \text { syst. } \pm \text { evol. }\end{array}$ \\
\hline \multicolumn{4}{|c|}{$2.75^{\circ}$ spectrometer } \\
\hline 0.017 & 1.2 & $-0.497 \pm 0.163 \pm 0.147$ & $-0.421 \pm 0.115 \pm 0.113 \pm 0.016$ \\
\hline 0.024 & 1.6 & $181 \pm 0.092 \pm 0.079$ & $-0.409 \pm 0.071 \pm 0.066 \pm 0.007$ \\
\hline 0.035 & 2.0 & $-0.345 \pm 0.073 \pm 0.044$ & $-0.304 \pm 0.061 \pm 0.039 \pm 0.005$ \\
\hline 0.049 & 2.6 & $-0.237 \pm 0.046 \pm 0.024$ & $-0.215 \pm 0.041 \pm 0.023 \pm 0.004$ \\
\hline 0.078 & 3.3 & $-0.127 \pm 0.033 \pm 0.014$ & $-0.117 \pm 0.031 \pm 0$. \\
\hline 0.123 & 4.1 & $-0.077 \pm 0.031 \pm 0.009$ & $-0.073 \pm 0.030 \pm 0.010 \pm 0.001$ \\
\hline 0.173 & 4.6 & $-0.071 \pm 0.033 \pm 0.009$ & $-0.069 \pm 0.033 \pm 0.010 \pm 0.001$ \\
\hline 0.241 & 5.1 & $-0.053 \pm 0.028 \pm 0.007$ & $-0.053 \pm 0.028 \pm 0.008 \pm 0.000$ \\
\hline 0.340 & 5.5 & $0.002 \pm 0.037 \pm 0.004$ & $0.001 \pm 0.036 \pm 0.004 \pm 0.000$ \\
\hline 0.423 & 5.8 & $0.028 \pm 0.061 \pm 0.008$ & $0.027=$ \\
\hline \multicolumn{4}{|c|}{$5.5^{\circ}$ Spectrometer } \\
\hline 0.084 & 5.5 & $-0.150 \pm 0.029 \pm 0.019$ & $-0.153 \pm 0$ \\
\hline 0.123 & 7.2 & $-0.113 \pm 0$ & $-0.121 \pm$ \\
\hline 0.172 & 8.9 & $-0.058 \pm 0.015 \pm 0.007$ & $-0.066 \pm 0.016 \pm 0.009 \pm 0.003$ \\
\hline 0.242 & 10.7 & $-0.041 \pm 0.012 \pm 0.005$ & $-0.047 \pm 0.012 \pm 0.006 \pm 0.003$ \\
\hline 0.342 & 12.6 & $-0.021 \pm 0.013 \pm 0.005$ & $-0.024 \pm 0.012 \pm 0.005 \pm 0.001$ \\
\hline 0.442 & 13.8 & $-0.011 \pm 0.014 \pm 0.003$ & $-0.011 \pm 0.012 \pm 0.003 \pm 0.001$ \\
\hline 0.564 & 15.0 & $0.005 \pm 0.012 \pm 0.002$ & $0.003 \pm 0.008 \pm 0.001 \pm 0.000$ \\
\hline
\end{tabular}

over the measured range in case of E154. However, the shape of the structure function at low $x$ affects the extrapolation to $x=0$. The effect of the perturbative evolution is qualitatively the same for the proton. It is smaller than for the neutron because the $x$ dependence of $g_{1}^{p}$ is more similar to $F_{1}^{p}$ than $g_{1}^{n}$ to $F_{1}^{n}$.

The data for $g_{1}^{n}$, evolved to $Q^{2}$ of $5 \mathrm{GeV}^{2}$ and averaged between the two spectrometers, are given in tab. 4.4. 


\section{Proton}

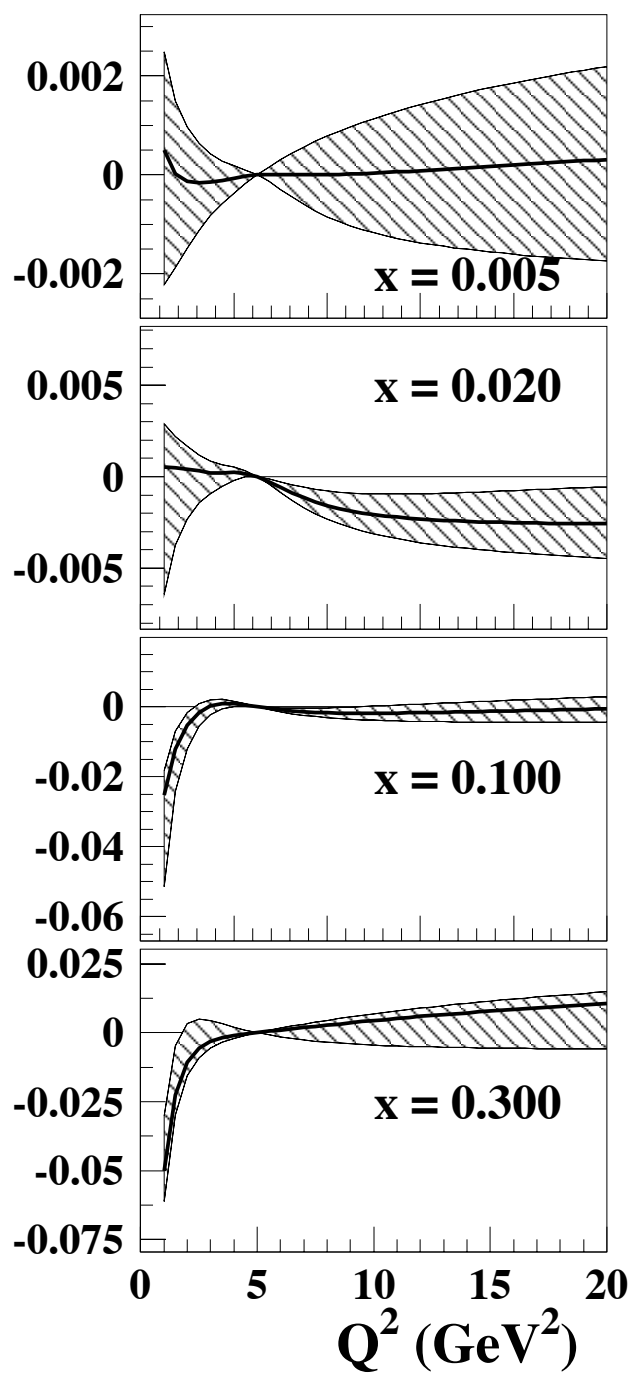

Neutron
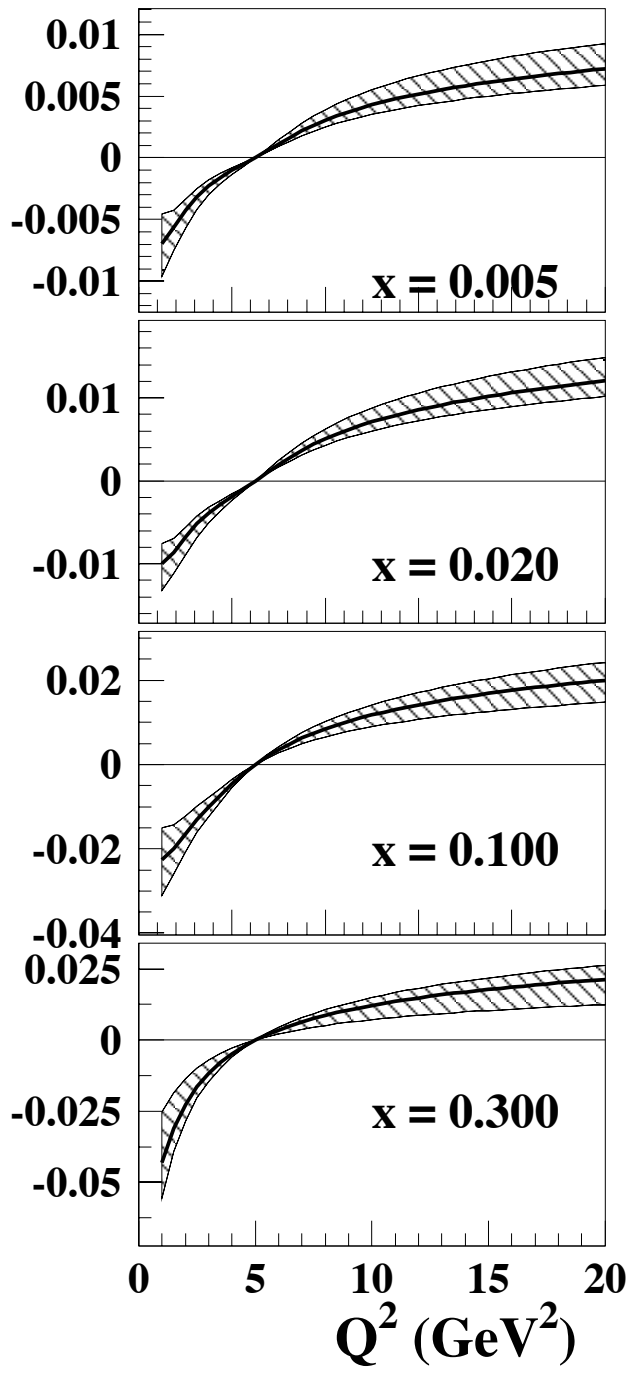

Figure 4.7: Evolution of the ratios $g_{1} / F_{1}$ for the proton (left) and the neutron (right). The difference $\frac{g_{1}}{F_{1}}\left(x, Q^{2}\right)-\frac{g_{1}}{F_{1}}\left(x, 5 \mathrm{GeV}^{2}\right)$ is plotted. The $\overline{\mathrm{MS}}$ fit is shown by solid lines, and the hatched area represents the total (experimental and theoretical) uncertainty. 
Table 4.4: Results for $g_{1}^{n}$ Evolved to $\left\langle Q^{2}\right\rangle=5 \mathrm{GeV}^{2}$ According to Eq. (4.71).

\begin{tabular}{crrr}
\hline$x_{i}$ & $\begin{array}{c}Q_{i}^{2} \\
\mathrm{GeV}^{2}\end{array}$ & \multicolumn{1}{c}{$\begin{array}{c}g_{1}^{n}\left(x_{i}, Q_{i}^{2}\right) \\
\text { stat. } \pm \text { syst. }\end{array}$} & \multicolumn{1}{c}{$\begin{array}{c}g_{1}^{n}\left(x_{i}, 5 \mathrm{GeV}^{2}\right) \\
\text { stat. } \pm \text { syst. } \pm \text { evol. }\end{array}$} \\
\hline 0.017 & 1.2 & $-0.352 \pm 0.115 \pm 0.109$ & $-0.419 \pm 0.115 \pm 0.109 \pm 0.014$ \\
0.024 & 1.6 & $-0.374 \pm 0.071 \pm 0.064$ & $-0.409 \pm 0.071 \pm 0.064 \pm 0.006$ \\
0.035 & 2.0 & $-0.289 \pm 0.061 \pm 0.038$ & $-0.304 \pm 0.061 \pm 0.038 \pm 0.005$ \\
0.049 & 2.6 & $-0.204 \pm 0.040 \pm 0.022$ & $-0.208 \pm 0.040 \pm 0.022 \pm 0.004$ \\
0.081 & 4.5 & $-0.137 \pm 0.021 \pm 0.016$ & $-0.136 \pm 0.021 \pm 0.016 \pm 0.002$ \\
0.123 & 6.6 & $-0.108 \pm 0.015 \pm 0.012$ & $-0.111 \pm 0.015 \pm 0.012 \pm 0.002$ \\
0.173 & 8.2 & $-0.061 \pm 0.014 \pm 0.009$ & $-0.067 \pm 0.014 \pm 0.009 \pm 0.003$ \\
0.242 & 9.8 & $-0.042 \pm 0.011 \pm 0.007$ & $-0.048 \pm 0.011 \pm 0.007 \pm 0.003$ \\
0.342 & 11.7 & $-0.017 \pm 0.011 \pm 0.005$ & $-0.021 \pm 0.011 \pm 0.005 \pm 0.001$ \\
0.441 & 13.3 & $-0.007 \pm 0.011 \pm 0.002$ & $-0.009 \pm 0.011 \pm 0.002 \pm 0.001$ \\
0.564 & 15.0 & $0.003 \pm 0.008 \pm 0.001$ & $0.003 \pm 0.008 \pm 0.001 \pm 0.000$ \\
\hline
\end{tabular}

For the integral of the neutron structure function in the measured range, we obtain (at $\left.Q^{2}=5 \mathrm{GeV}^{2}\right)$

$$
\int_{0.014}^{0.7} d x g_{1}^{n}(x)=-0.035 \pm 0.003 \pm 0.005 \pm 0.001,
$$

where the first error is statistical, the second is systematic, and the third is due to the uncertainty in the evolution. This value agrees well with the number $-0.036 \pm 0.004$ (stat.) \pm 0.005 (syst.) obtained assuming the $Q^{2}$ independence of $g_{1}^{n} / F_{1}^{n}$.

\subsection{Revised low $x$ extrapolation}

In the singlet sector, the data are compatible with small values of $\alpha_{Q}$, and the uncertainties in $\alpha_{Q}$ and $\alpha_{G}$ are large. Note that the low $x$ behavior of the valence distributions is reasonably convergent, $\Delta u_{V}(x) \sim x^{0.18_{-0.10}^{+0.37}}$ and 
$\Delta d_{V}(x) \sim x^{-0.43_{-0.12}^{+0.77}}$ as $x \rightarrow 0$, and is consistent with the Regge predictions [57] at low $Q^{2} \approx Q_{\circ}^{2}=0.34 \mathrm{GeV}^{2}$. One should notice that the convergent behavior of the structure functions at low $x$ and high $Q^{2}$ would be incompatible with the pQCD predictions $[168,169]$. In fact, the polarized parton distributions, and therefore $g_{1}$, are expected to rise faster than any power of $\log (1 / x)$, but slower than any power of $x$, even if the initial distributions at low $Q_{\circ}^{2}$ scale are convergent. At sufficiently low $x$ (and/or high $Q^{2}$ ), the singlet distributions start to dominate, and both the proton and neutron structure functions have the same asymptotic behavior. The contributions from the valence quarks $g_{1}^{n}$ valence $=(1 / 18) C_{q} \otimes\left(\Delta u_{V}+4 \Delta d_{V}\right)$ and sea quarks and gluons $g_{1}^{n \text { sea+gluon }}=(1 / 3) C_{q} \otimes \Delta \bar{q}+(1 / 9) C_{G} \otimes \Delta G$ to the neutron spin structure function at $Q^{2}=5 \mathrm{GeV}^{2}$ are shown in fig. 4.8. One can see that the sea and gluon contributions exceed (by the absolute value) the valence contributions around $x \approx 10^{-3}$. This is the reason for the $g_{1}^{p}$ sign change around this $x$ value (see fig. 4.9) mentioned above. Although the sea contributions to $g_{1}^{n}$ are relatively modest in the E154 data range $x>0.01$, the strong $x$ dependence $g_{1}^{n} \sim x^{-0.81 \pm 0.15}$ of the NLO evolved E154 data below $x=0.1$ is largely due to the sea and gluon contributions.

Using E154 results and the $\overline{\mathrm{MS}}$ parametrization for the contributions from the unmeasured low and high $x$ regions, we determine the integral of $g_{1}^{n}$ at $5 \mathrm{GeV}^{2}$,

$$
\Gamma_{1}^{n}\left(5 \mathrm{GeV}^{2}\right)=-0.058 \pm 0.004 \pm 0.007 \pm 0.007
$$

where the errors are statistical, systematic and due to the extrapolation. 


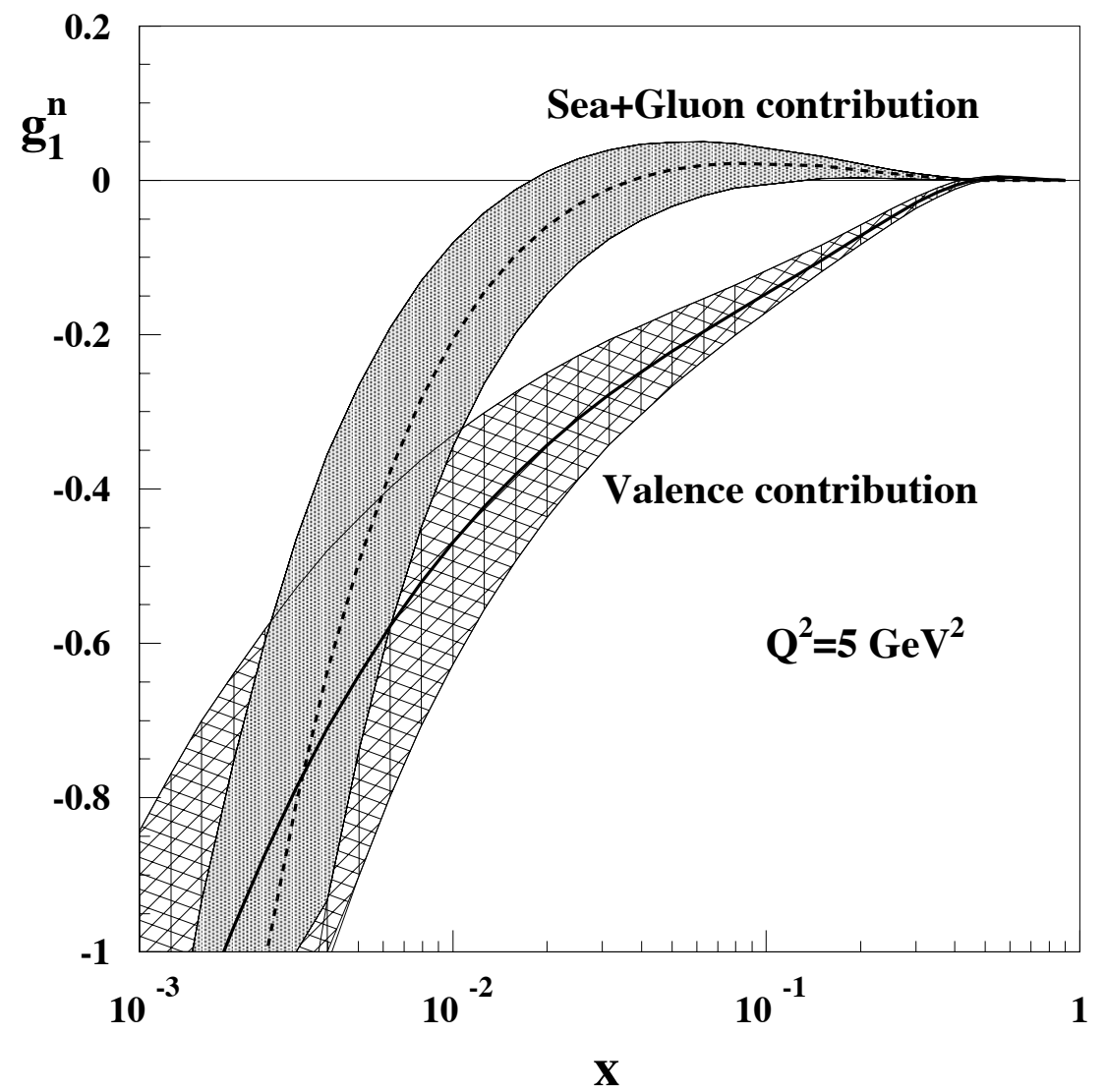

Figure 4.8: The contributions to the structure function $g_{1}^{n}$ of the neutron from the valence quarks (solid line) and from the sea quarks and gluons (dashed line). The shaded and hatched areas represent the total uncertainties on each quantity.

The behavior of the purely non-singlet combination $\left(g_{1}^{p}-g_{1}^{n}\right)(x)$ is expected to be softer at low $x$ than its singlet counterpart [128, 129]. Evolving the E154 neutron and E143 [18] proton data to $Q^{2}=5 \mathrm{GeV}^{2}$ and using the 


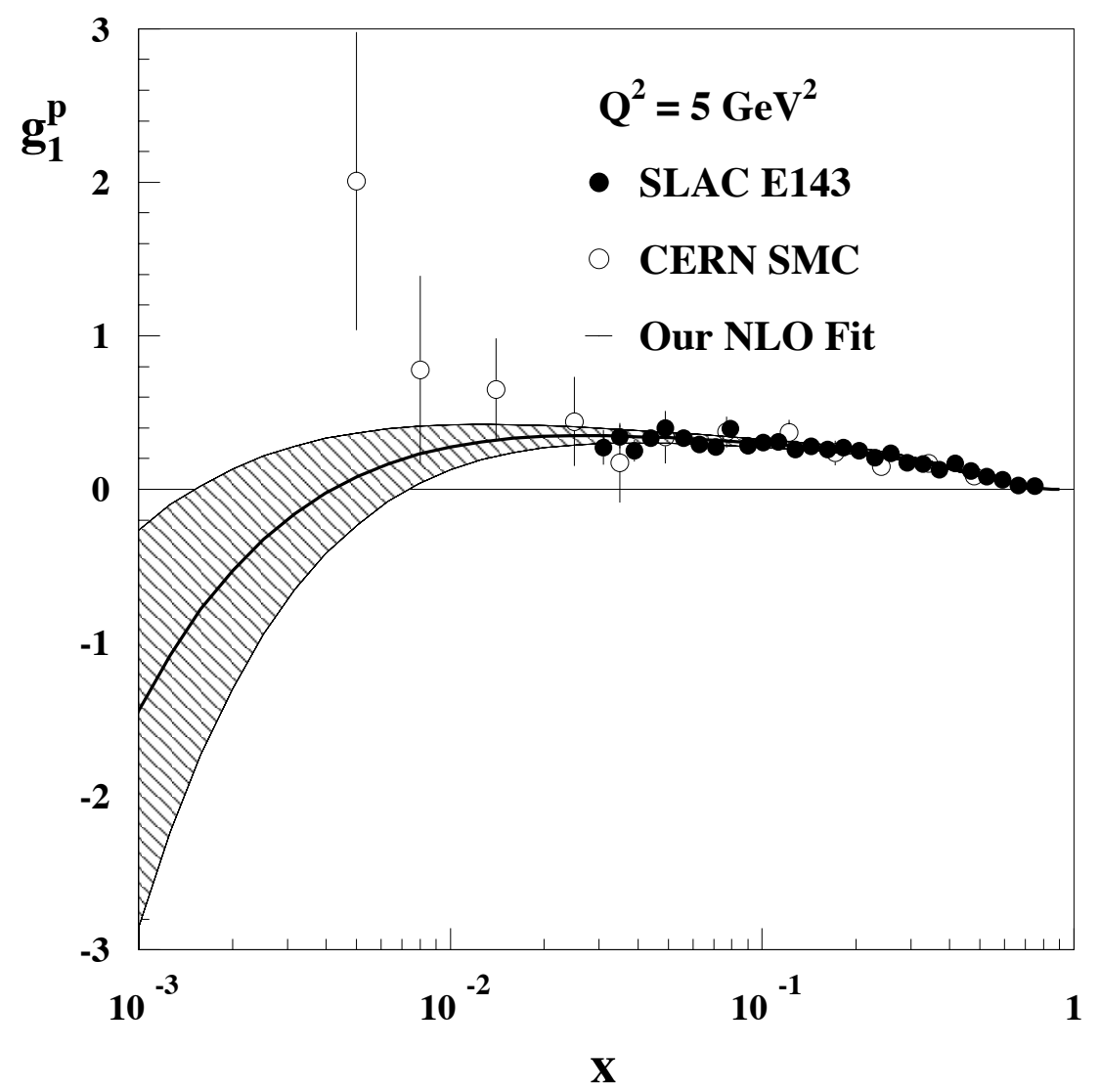

Figure 4.9: The $g_{1}^{p}$ structure function at $Q^{2}=5 \mathrm{GeV}^{2}$.

$\overline{\mathrm{MS}}$ parametrization of tab. 4.1, we obtain for the Bjorken sum

$$
\Gamma_{1}^{p-n}\left(5 \mathrm{GeV}^{2}\right)=\int_{0}^{1} \mathrm{dx}\left(\mathrm{g}_{1}^{\mathrm{p}}-\mathrm{g}_{1}^{\mathrm{n}}\right)=0.172 \pm 0.004 \pm 0.010 \pm 0.007
$$

where the first error is statistical, the second is systematic, and the third is due to the uncertainty in the evolution. This value is in good agreement with the $O\left(\alpha_{s}^{3}\right)$ [48] prediction 0.186 evaluated with $\alpha_{s}\left(M_{Z}\right)=0.109$, and it also agrees very well with the result in tab. 4.2 obtained by direct integra- 
tion of the parton densities. The result is fairly insensitive to the details of the low $x$ extrapolation, which is well constrained by the data. The low $x$ behavior in the non-singlet polarized sector is also relatively insensitive to the higher order corrections [170]. On the other hand, the low $x$ extrapolation of the proton and neutron integrals alone still relies on the assumption that the asymptotic behavior of the sea quarks and gluon distributions can be determined from the present data, and that the effects of higher-order re-summations are small. These assumptions, and therefore the evaluation of the total quark helicity $\Delta \Sigma$, are on potentially weaker grounds. Precise higher energy data on the polarized structure functions of both proton and neutron are required to determine this quantity. 


\section{CHAPTER 5}

\section{SUMMARY AND OUTLOOK}

The E154 data constitute the most precise measurement of the $g_{1}^{n}$ structure function. The higher energy of the beam and the smaller values of the spectrometer angles allowed us to extend the kinematic coverage of the previous SLAC E142 experiment. The values of $x$ were extended down to 0.014 (compared to 0.03 of E142) at the average $Q^{2}$ of $5 \mathrm{GeV}^{2}$ (compared to $2 \mathrm{GeV}^{2}$ ). We found large and strongly $x$ dependent values for

$g_{1}^{n}\left(x,\left\langle Q^{2}\right\rangle=5 \mathrm{GeV}^{2}\right)$ at low $x$ that called into question the traditional Regge theory method for extrapolating to $x=0$ needed for evaluation of the sum rules. We turned to perturbative QCD and performed a NLO global fit to all the polarized DIS structure date. We found that the low $x$ behavior and the $Q^{2}$ evolution are closely related in the measured region. With the low and high $x$ NLO fit extrapolations, the Bjorken Sum Rule was evaluated and confirmed within one sigma error. We could see that the helicity distributions of the valence quarks (non-singlet distributions) are well determined 
by the measurements. On the other hand the singlet distributions, the sea quarks and gluons, are only qualitatively constrained. The effort of the spin community should be focussed on the coverage of a wider kinematic range of measurements to lower (and higher) $x$ and higher $Q^{2}$ values to give a better leverage for the pQCD evolution, and on measurements directly sensitive to the sea quark and gluon distributions.

The nucleon spin structure program continues, and with the addition of the polarized collider experiments to the fixed target ones, promises new, exciting measurements.

The SMC collaboration at CERN is analyzing the inclusive proton data obtained in the 1996 run and the semi-inclusive data from the runs of last and previous years. Preliminary results have been presented for the $g_{1}^{p}$ structure function [52] and the asymmetries from the semi-inclusive analysis [171]. The semi-inclusive measurements tag the leading hadron and assure that the detected event is a deep inelastic one. If only those events are counted for the determination of the $g_{1}^{p}$ structure function, then the radiative corrections do not contain elastic and quasi-elastic tails. These tails are significant contributors, specially at low $x$ of the SMC kinematic region, to the dilution of asymmetry due to the radiative corrections. The new method of analysis will improve the statistical precision of the measurement (smaller dilution due to radiative tails) by $10-40 \%[171]$.

The SLAC E155 experiment recently finished taking data for the structure function $g_{1}$ of the proton and the deuteron. It used the $50 \mathrm{GeV}$ polarized 
electron beam, the same detector system as E154 with the addition of $10.5^{\circ}$ angle (relative to the beam direction) spectrometer, and the ammonia and ${ }^{6} \mathrm{LiD}$ targets similar to those of E143. It has the same $x$ range as E154 and increased (due to the new spectrometer) $Q^{2}$ coverage.

The HERMES collaboration at DESY, that is now collecting data for the $g_{1}^{p}$, shifts to semi-inclusive scattering to be directly sensitive to the sea quark distributions [172]. There are also plans to measure the gluon spin distribution from the asymmetries of the open charm productions.

The COMPASS experiment at CERN has been approved to measure $\Delta G / G$ via open charm production [173]. It will also continue the semiinclusive program started by SMC.

The RHIC program at BNL [174] will measure $\Delta G / G$ in the polarized $\vec{p}-\vec{p}$ collisions from prompt photon production $q+g \rightarrow q+\gamma$ tagged by high $p_{T} \gamma$ 's. It will also probe the diversity of flavors from the single and double asymmetries of the Drell-Yan processes with weak bosons (like $\vec{p}+\vec{p} \rightarrow$ $\left.W^{ \pm}+X \rightarrow l \bar{l}+X\right)$.

CEBAF experiment [175] will measure the $A_{1}^{n}$ asymmetry at high $x$ to check the pQCD prediction that $A_{1}(x) \rightarrow 1$ as $x \rightarrow 1$.

The SLAC open charm proposal [176] aims at $\Delta G / G$ via open charm production.

The polarization of the HERA proton beam at DESY is now under consideration [177]. The $g_{1}$ structure functions could be measured there in the kinematic region of the present unpolarized data: down to $x=10^{-5}$ and up 
to $Q^{2}=10^{4} \mathrm{GeV}^{2}$. Also $\Delta G / G$ could be measured in heavy quark production processes with two high $p_{T}$ jets or two high $p_{T}$ hadron tags.

The HERA wide kinematic coverage could be supplemented by possible polarized Next Linear Collider (NLC) fixed target experiments.

There is also a possibility of polarizing the Main Injector at Fermilab for the polarized Drell-Yan program.

The new data will test the polarized pQCD at the level of precision of the present day unpolarized measurements. The author hopes the future results will create the excitement and stimulate the theoretical and experimental progress as the EMC "spin crisis" experiment did. 


\section{REFERENCES CITED}

[1] M.Gell-Mann, Phys. Lett. 8, 214 (1964)

[2] G.Zweig, CERN-TH-401 (1964)

G.Zweig, CERN-TH-412 (1964)

[3] E.D.Bloom et al., Phys. Rev. Lett. 23, 930 (1969)

M.Breidenback et al., Phys. Rev. Lett. 23, 935 (1969)

[4] J.D.Bjorken, Phys. Rev. 179, 1547 (1969)

[5] J.D.Bjorken, E.A.Paschos, Phys. Rev. 185, 1975 (1969)

[6] R.P.Feynman, Phys. Rev. Lett. 23, 1415 (1969)

[7] C.N.Yang, R.L.Mills, Phys. Rev. 96, 191 (1954)

[8] G.t'Hooft, Proc. Colloquium on Renormalization of Yang-Mills Fields and Applications to Particle Physics Marseilles, (1972), ed. C.P.Korthals-Altes

[9] D.J.Gross, F.Wilczek, Phys. Rev. Lett. 30, 1343 (1973)

[10] H.D.Politzer, PRL 30, 1346 (1973)

[11] M.Han, Y.Nambu, Phys. Rev. 139B, 1006 (1965)

[12] H.Fritzsch, M.Gell-Mann, Proc. XVI Int. Conf. on High Energy Physics, Fermilab (1972), eds. J.D.Jackson, A.Roberts

[13] CERN Courier, V30, No.9, 1 (Dec. 1990) 
[14] The E80 Collab., M.J.Alguard et al., Phys. Rev. Lett. 37, 1258 (1976) The E80 Collab., M.J.Alguard et al., Phys. Rev. Lett. 37, 1261 (1976) The E80 Collab., M.J.Alguard et al., Phys. Rev. Lett. 41, 70 (1978)

[15] The E130 Collab., G.Baum et al., Phys. Rev. Lett. 51, 1135 (1983)

[16] The EMC Collab., J.Asham et al., Phys. Lett. B206, 364 (1988) The EMC Collab., J.Asham et al., Nucl. Phys. B328, 1 (1990) V.Hughes et al., Phys. Lett. B21 2, 511 (1988)

[17] The E142 Collab., P.L.Anthony et al., Phys. Rev. Lett. 71, 959 (1993) The E142 Collab., P.L.Anthony et al., Phys. Rev. D54, 6620 (1996)

[18] The E143 Collab., K.Abe et al., Phys. Rev. Lett. 74, 346 (1995)

[19] The E143 Collab., K.Abe et al., Phys. Rev. Lett. 75, 25 (1995)

[20] The SMC Collab., D.Adams et al., Phys. Lett. B329, 399 (1994) preprint hep-ex/9702005 (1997), submitted to Phys. Rev. D

[21] The SMC Collab., B.Adeva et al., Phys. Lett. B302, 533 (1993) The SMC Collab., D.Adams et al., Phys. Lett. B357, 248 (1995) The SMC Collab., D.Adams et al., CERN-PPE/97-008 , (1997), submitted to Phys. Lett. B.

[22] The HERMES Collab., K.Ackerstaff et al., preprint hep-ex/9702005 (1997), submitted to Phys. Lett. B

[23] T.Pussieux, R.Windmolders, Symposium on the Internal Structure of the Nucleon, p212, World Scientific 1995, ed. by V.W.Hughes, C.Cavata

[24] L.W.Whitlow et al., Phys. Lett. B250, 193 (1990)

[25] S.Dasu et al., Phys. Rev. D49, 5641 (1994)

[26] F.E.Close, An Introduction to Quarks and Partons, Academic Press (1981)

[27] J.L.Friar et al., Phys. Rev. C42, 2310 (1990)

[28] C.Ciofi degli Atti et al., Phys. Rev. C48, 968 (1993) 
[29] J.Aubert et al., Phys. Lett. B123, 275 (1983)

[30] S. Weinberg, Phys. Rev. 150, 1313 (1966)

[31] C.Callan, D.Gross, Phys. Rev. Lett. 22, 156 (1969)

[32] J.Kuti, V.F.Weisskopf, Phys. Rev. D4, 3418 (1971)

[33] N.Isgur, G.Karl, R.Koniuk, Phys. Rev. Lett. 41, 1269 (1978)

[34] Z.Dziembowski, C.J.Martoff, P.Zyla, Phys. Rev. D50, 5613 (1994)

[35] J.S.Bell, R.Jackiw, Nuovo Cim. 60A, 47 (1967)

S.L.Adler, Phys. Rev. 177, 2426 (1969)

[36] K.Fujikawa, Phys. Rev. Lett. 42, 1195 (1979)

[37] M.Anselmino, A.Efremov, E.Leader, Phys. Rept. 261, 1 (1995)

[38] R.D.Carlitz, J.D.Collins, A.H.Mueller, Phys. Lett. B214, 229 (1988)

[39] S.J.Brodsky, G.P.Lepage Proceedings of the 1979 SLAC Summer Institute on Particle Physics, SLAC (1979)

[40] R.L.Jaffe, A.Manohar, Nucl. Phys. B337, 505 (1990)

S.J.Brodsky, J.Ellis, M.Karliner, Phys. Lett. B206, 309 (1988)

[41] P.G.Ratcliffe, Phys. Lett. B192, 180 (1987)

[42] X.Ji, J.Tang, P.Hoodbhoy, Phys. Rev. Lett. 76, 740 (1996)

[43] X.Ji, Phys. Rev. Lett. 78, 610 (1997)

$X . J i$ hep-ph/9610369

[44] R.L.Jaffe, h e, - (p)ph/9603422

[45] D.J.Gross, F.Wilczek, Phys. Rev. D9, 1974 (980)

[46] J.D.Bjorken, Phys. Rev. 148, 1467 (1966)

J.D.Bjorken, Phys. Rev. D1, 1376 (1970)

[47] A.J.G.Hey Proceedings of the 9th Rencontre de Moriond, ed. J.Tran Thanh Van (1974) 
[48] S.G.Gorishny, S.A.Larin, Phys. Lett. B172, 109 (1986)

S.A.Larin, J.A.M. Vermaseren, Phys. Lett. B259, 345 (1991)

[49] J.Kodaira et al., Phys. Rev. D20, 627 (1979)

J.Kodaira et al., Nucl. Phys. B159, 99 (1979)

[50] F.E.Close, R.G.Roberts, Phys. Lett. B316, 165 (1993)

[51] J.Ellis, R.L.Jaffe, Phys. Rev. D9, 1444 (1974)

J.Ellis, R.L.Jaffe, Phys. Rev. D10, 1663 (1974)

[52] A.Magnon, Talk presented at DIS97, Chicago (1997), to be published in the proceedings

[53] The E143 Collab., K.Abe et al., Phys. Rev. Lett. 76, 587 (1996)

[54] The SMC Collab., B.Adeva et al., Phys. Lett. B336, 125 (1994)

[55] S. Wandzura, F. Wilczek, Phys. Lett. 72B, 195 (1977)

[56] H.Burkhardt, W.N.Cottingham, Ann. Phys. 56, 453 (1970)

[57] R.L.Heimann, Nucl. Phys. B64, 429 (1973)

[58] J.F.Gunion Phys. Rev. D10, 242 (1974)

R.Blankenbecler, S.J.Brodsky Phys. Rev. D10, 2973 (1974)

G.R.Farrar, D.R.Jackson Phys. Rev. Lett. 35, 1416 (1975)

S.J.Brodsky, M.Burkardt, I.Schmidt Nucl. Phys. B441, 197 (1995)

[59] A.Abragam, M.Goldman, Phys. Rep. Prog. 41, 385 (1978)

[60] The E143 Collab., K.Abe et al., Phys. Lett. B364, 61 (1995)

[61] A.A.Sokolov, I.M.Ternov, Sov. Phys. Doklady 8, 1203 (1964)

[62] J.Ellis, M.Karliner Preprint CERN-TH/95-279, hep-ph/9510402

[63] T.Maruyama, et al. SLAC-PUB-92-322 (1992)

[64] R.Alley et al., Nucl. Instrum. Methods A365, 1 (1995)

[65] M.Woods SLAC-PUB-7320 (1996) 
[66] Z.D.Farkas, et al. SLAC-PUB-1453 (1974)

[67] F.-J.Decker, et al. SLAC-PUB-7214 (1996)

[68] R.Erickson, et al., SLAC-PUB-7177 (1996)

[69] J.D.Jackson, Classical Electrodynamics, John Wiley and So., (1975)

[70] P.Cooper et al., Phys. Rev. Lett. 42, 1386 (1979)

[71] Review of Particle Properties, PDG (R.M.Barnett et al.), Phys. Rev. D54, (1996)

[72] L.Stuart, E154 Tech. Note \#6, (1995), unpublished

[73] C.Young, E154 Tech. Note \#35, (1996), unpublished

[74] C.Møller, Ann. Phys. (Leipzig) 14, 532 (1932)

[75] H.R.Band, et al., E154 Tech. Note \#40, (1996), unpublished

[76] H.R.Band, AIP Conf. Proc. 343, 245 (1994)

[77] H.R.Band, et al., E143 Tech. Note \#110, (1996), unpublished

[78] L.D.Landau, E.M.Lifshitz, Quantum Mechanics: non-relativistic theory, Pergamon Press, (1977)

[79] G.G.Scott, H.W.Sturner, Phys. Rev. 184, 184 (1969)

[80] M.Swartz, et al., Nucl. Instrum. Methods A363, 526 (1995)

[81] L.G.Levchuk, Nucl. Instrum. Methods A345, 496 (1994)

[82] H.Middleton Ph.D. thesis, Princeton University (1994), unpublished

[83] M.V.Romalis, $\mathrm{PhD}$ thesis, Princeton University (1997), unpablished

[84] W.Happer, Rev. Mod. Phys. 44, 169 (1972)

[85] R.L.Gramblin, T.R.Carver, Phys. Rev. A138, 946 (1965)

[86] T.E.Chupp, et al., Phys. Rev. C36, 2244 (1987) 
[87] M.V.Romalis, E154 Tech. Note \#34, (1996), unpublished

[88] M.V.Romalis, T.B.Smith, E154 Target Polarization Tech. Note, (1996), unpublished

[89] G.G.Petratos, Report SLAC/ESA-2/93 (1993), unpublished

[90] R.Arnold, et al., Report SLAC/ESA-4/93 (1993), unpublished

[91] Yu.G.Kolomensky, PhD thesis, University of Massachusetts (1997), unpablished

[92] D.M.Kawall, PhD thesis, Stanford University (1995), unpablished

[93] L.W.Whitlow, SLAC-PUB-0357 (1990)

[94] E.R.Hayes, et al., Aragone ANL-6916 (1964)

[95] P.Baillon, et al., Nucl. Instr. Meth. A276, 492 (1989)

[96] R.J.Apsimon, et al., Nucl. Instr. Meth. A241, 339 (1995)

[97] Y.Tomkiewicz, E.L.Garwin, SLAC-PUB-1356 (1973)

[98] E.L.Garwin, Y.Tomkiewicz, D.Trines, Nucl. Instr. Meth. 107, 365 (1973)

[99] T.Hayashi, Hammamatsu Photonics K.K. Application, RES-0791-02

[100] A.M.Gorin, et al., Nucl. Instr. Meth. A251, 461 (1986)

[101] P.J.Carlson, Nucl. Instr. Meth. 158, 403 (1979)

[102] P.Baillon, et al., Nucl. Instr. Meth. A126, 13 (1975)

[103] J.Xu, PhD thesis, Syracuse University (1994), unpablished

[104] H.Borel, et al., IEEE Trans. Nucl. Sci. Vol.42, No.4, 529 (1995)

H.Borel et al., Erratum, IEEE Trans. Nucl. Sci. Vol.42, No.6, 8347 (1995)

[105] N.V.Rabin, Inst. Exp. Tech. 35, 947 (1992)

[106] REngelmann, et al., Nucl. Instr. Meth. 216, 45 (1983) 
[107] P.L.Anthony, Z.M.Szalata, SLAC-PUB-7201 (1996)

[108] X.J.Wang, E154 collaboration meeting, May 1996, unpublished

[109] P.Zyla, E154 Tech. Note \#24, (1995), unpublished

[110] L.Sorrell, E154 Tech. Note \#25, (1995), unpublished

[111] Yu.Kolomensky, M.Romalis, E154 Technical Note \#48 (1996), unpablished

[112] Yu.Kolomensky, M.Olson, P.Zyla, E154 Technical Note (1997), unpablished

[113] Yu.Kolomensky, F.Sabatie, E154 Technical Note \#44 (1996), unpablished

[114] Yu.G.Kolomensky, E154 collaboration meeting, May 1996, unpublished

[115] P.E.Bosted, E154 Tech. Note \#29, (1995), unpublished

[116] T.V.Kuchto, N.M.Schumeiko, Nucl. Phys. B219, 412 (1982)

[117] I.V.Akushevich, N.M.Schumeiko, J. Phys G20, 513 (1994)

[118] I.V.Akushevich, N.M.Schumeiko, A.E.Tolkachev POLRAD 1.5 (1996), unpublished

[119] L.Stuart, private communication

[120] B.Blankleider, R.M.Woloshyn, Phys. Rev. C29, 538 (1984)

[121] L.W.Mo, Y.S.Tsai, Rev. Mod. Phys. 41, 205 (1969)

[122] Y.S.Tsai, SLAC-PUB-884 (1971)

[123] D.Y.Bardin, N.M.Schumeiko, Nucl. Phys. B127, 242 (1977)

[124] M.Arneodo et al., Phys. Lett. B364, 107 (1995)

[125] J.Ellis, M.Karliner, Phys. Lett. B213, 73 (1988)

[126] A.Donnachie, P.V.Landshoff, Phys. Lett. B296, 227 (1992) 
[127] B.Badelek, et al., DESY 96-049, hep-ph/9604345 (1996)

R.D.Ball, A.De Roeck hep-ph/9609309, unpublished

[128] J.Bartels, B.I.Ermolaev, M.G. Ryskin, Z. Phys. C72, 627 (1996)

[129] J.Bartels, B.I.Ermolaev, M.G. Ryskin, Z. Phys. C70, 1996 (273)

[130] R.Mertig, W.L. van Neerven, Z. Phys. C70, 637 (1996), hep$\mathrm{ph} / 9506451$ (revised version)

W.Vogelsang, Phys. Rev. D54, 2023 (1996)

[131] M. Gluck, et al., Phys. Rev. D53, 4775 (1996), hep-ph/9508347

[132] R.D.Ball, S.Forte, G.Ridolfi, Phys. Lett. B378, 255 (1996)

[133] T.Gehrmann, W.J.Stirling, Phys. Rev. D53, 6100 (1996)

[134] G. Altarelli, et al., hep-ph/9701289 (1997)

[135] H.Georgi, H.D.Politzer, Phys. Rev. D9, 416 (1974)

[136] K.Wilson, Phys. Rev. 179, 1499 (1969)

[137] V.N.Gribov, L.N.Lipatov, Sov. J. Nucl. Phys. 15, 438, 675 (1972)

Yu.L.Dokshitzer Sov. Phys. JETP 46, 461 (1977)

[138] G.Altarelli, G.Parisi Nucl. Phys. B126, 298 (1977)

[139] J.M.Namyslowski in 'Progress in prticle and nuclear physics', Vol.14, ed. by A.Faessler, Pergamon Press, 49 (1995)

[140] G.P.Lepage, S.J.Brodsky Phys. Rev. D22, 2157 (1980)

[141] E.Bloom, F.Gilman Phys. Rev. Lett. 25, 1140 (1970)

[142] S.D.Drell, T.-M.Yan Phys. Rev. Lett. 24, 181 (1970)

[143] A.Lung, et al. Phys. Rev. Lett. 70, 718 (1993)

[144] P.D.B.Collins An Introduction to Regge Theory and High Energy Physics, Cambridge University Press (1977)

D.C.Cheng, G.K.O'Neil Elementary Particle Physics, Addison-Wesley Publishing Company (1979) 
B.L.Ioffe, V.A.Khose, L.N.Lipatov Hard Processes, Vol.1, North Holland (1983)

[145] J.Kuti Internal Spin Structure of the Nucleon, (ed. by V.W.Hughes, C.Cavata) 134 (1995)

J.Kuti preprint MIT CTP \#234 (1971)

[146] S.D.Bass, P.V.Landshoff Phys.Lett. B336, 537 (1994)

[147] F.E.Close, R.G.Roberts Phys.Lett. B336, 257 (1994)

[148] P.V.Landshoff "Zuoz 1994, Proceedings, Hadronic Aspects of Collider Physics", 135 (1994), hep-ph/9410250

[149] M.Gluck, E.Reya, A.Vogt Z. Phys. C67, 433 (1995)

[150] M.Lacombe et al. Phys. Rev. C21, 861 (1980)

M.J.Zuilhof, J.A.Tjon Phys. Rev. C22, 2369 (1980)

R.R. Machleid et al. Phys. Rep. 149, 1 (1987)

[151] The E154 Collab., K.Abe et al., SLAC-PUB-7459, hep-ex/9705012, to be published in Phys. Rev. Lett.(1997)

[152] The E154 Collaboration, K. Abe et al., SLAC-PUB-7461, hepph/9705344, to be published in Phys. Lett.(1997)

[153] D.Graudenz, M.Hampel, A.Vogt, Ch.Berger, Z. Phys. C70, 77 (1996), hep-ph/9506333

[154] M.Gluck, E.Reya, A.Vogt, Z. Phys. C48, 471 (1990)

[155] M.A.Ahmed, C.G.Ross, Nucl. Phys. B111, 441 (1976)

[156] T.Muta Foundations of Quantum Chromodynamics, World Scientific (1987)

[157] S.J.Brodsky, H.J.Lu, Phys. Rev. D51, 3652 (1995)

[158] G.T.Bodwin, J.Qiu, Phys. Rev. D41, 2755 (1990)

[159] G.t'Hooft, M.Veltman, Nucl. Phys. B44, 189 (1972)

[160] W.Furmanski, R.Petronzio, Z. Phys. C11, 293 (1982) 
[161] G.Altarelli, G.Ross, Phys. Lett. B212, 391 (1988)

A.V.Efremov, O.V.Teryaev JINR-E2-88-287 (1988)

A.V.Efremov, O.V.Teryaev, Phys. Lett. B240, 200 (1990)

[162] S.Adler, W.Bardeen, Phys. Rev. 182, 1517 (1969)

[163] J.Kodaira, Nucl. Phys. B165, 129 (1980)

[164] P.Ratcliffe, Phys. Lett. B192, 309 (1987)

[165] CERN Application Software Group, CERN Program Library Long Writeup D506, (1992)

[166] A.D.Martin, R.G.Roberts, W.J.Stirling, Phys. Lett. B387, 419 (1987)

[167] S.J.Brodsky et al., Nucl. Phys. B369, 519 (1992)

[168] M.A.Ahmed, G.G.Ross, Phys. Lett. B56, 385 (1975)

M.B.Einhorn, J.Soffer, Nucl. Phys. B74, 714 (1986)

[169] R.D.Ball, S.Forte, G.Ridolf, Nucl. Phys. B444, 287 (1996)

[170] J.Blümlein, S.Riemersma, A.Vogt, Nucl. Phys. Proc. Suppl. 51C, 30 (1996)

J.Blümlein, S.Riemersma, A.Vogt, preprint DESY-96-131, (1996)

[171] E.Kabuss, Talk presented at DIS97, Chicago (1997), to be published in the proceedings

[172] P.Schüler, Talk presented at DIS97, Chicago (1997), to be published in the proceedings

[173] COMPASS proposal, CERN/SPSLC-96-14 (1996)

A.Bravar, Talk presented at DIS97, Chicago (1997), to be published in the proceedings

[174] G.Bunce et al., Particle World, Vol.3, 1 (1992)

[175] Z.-E.Meziani, et al., CEBAF proposal (1994)

[176] P.Bosted, et al., E156 SLAC proposal (1997) 
[177] A.De Roeck, Talk presented at DIS97, Chicago (1997), to be published in the proceedings

R.D.Ball, et al., Talk presented at 1996 HERA Workshop (1996), to be published in the proceedings; hep-ph/9609515

[178] P.Zyla, T.D.Averett, E154 Tech. Note \#43, (1996), unpublished

[179] J.K.Tuli: Nuclear Wallet Cards, July 1995

[180] Smirnov: Phys Let B364, 87-92 (1995)

[181] G.Curci, W.Furmanski, R.Petronzio, Nucl Phys. B175, 27 (1980)

[182] G. Altarelli, Proc. of the 27th Int. E. Majorana Summer School of Subnuclear Physics, Erice 1989, Plenum Press 1990 [CERN-TH.5675/90]; Proc. of the HERA-Workshop, Hamburg 1991, eds. W. Buchmüller and G. Ingelman, DESY, 1992, vol. I, p.379.

[183] E. Reya, Schladming lectures 1993, Springer Lecture Notes in Physics 426, 175 (1994).

[184] L.Mankiewicz, A.Schäfer, Phys. Lett. B242, 455 (1990)

L.Mankiewicz, Phys. Rev. D43, 64 (1991)

W.Vogelsang, Z. Phys. C50, 275 (1991)

[185] G.Altarelli, B.Lampe, Z. Phys. C47, 315 (1990)

[186] H.-Y.Cheng, Chin. J. Phys. 35, 25 (1997), hep-ph/9512267

[187] W.H.Press, A.Teukolsky, W.T.Vetterling, B.P.Flannery Numerical Recipies in C, Second edition, Cambridge U.P. (1992)

[188] CERN Application Software Group, CERN Program Library Short Writeup D107, (1992)

[189] E.G.Floratos, C.Kounnas, R.Lacaze, Nucl. Phys. B192, 417 (1981)

[190] CERN Application Software Group, CERN Program Library Short Writeup C306, (1992)

[191] G.Altarelli, P.Nason, G.Ridolfi, Phys. Lett. B320, 152 (1994)

ERRATUM, Phys. Lett. B325, 538 (1994) 
[192] C.Bourrely etal, CPT-96-PE-3327, hep-ph/9604204 (1996)

[193] W.Vogelsang, private communication 


\section{BIBLIOGRAPHY}

Abe K. et al., The E143 Collab., Phys. Lett. B364, 61 (1995)

Abe K. et al., The E143 Collab., Phys. Rev. Lett. 74, 346 (1995)

Abe K. et al., The E143 Collab., Phys. Rev. Lett. 75, 25 (1995)

Abe K. et al., The E143 Collab., Phys. Rev. Lett. 76, 587 (1996)

Abe K. et al., The E154 Collab., SLAC-PUB-7459, hep-ex/9705012, to be published in Phys. Rev. Lett. (1997)

Abe K. et al., The E154 Collaboration, SLAC-PUB-7461, hep-ph/9705344, to be published in Phys. Lett.(1997)

Abragam A., Goldman M., Phys. Rep. Prog. 41, 385 (1978)

Ackerstaff K. et al., The HERMES Collab., preprint hep-ex/9702005 (1997), submitted to Phys. Lett. B

Adams D. et al., The SMC Collab., Phys. Lett. B357, 248 (1995)

Adams D. et al., The SMC Collab., CERN-PPE/97-008 , (1997), submitted to Phys. Lett. B.

Adams D. et al., The SMC Collab., Phys. Lett. B329, 399 (1994)

Adams D. et al., The SMC Collab., preprint hep-ex/9702005 (1997), submitted to Phys. Rev. D 
Adeva B. et al., The SMC Collab., Phys. Lett. B302, 533 (1993)

Adeva B. et al., The SMC Collab., Phys. Lett. B336, 125 (1994)

Adler S.L., Phys. Rev. 177, 2426 (1969)

Adler S., Bardeen W., Phys. Rev. 182, 1517 (1969)

Ahmed M.A., Ross G.G., Phys. Lett. B56, 385 (1975)

Ahmed M.A., Ross C.G., Nucl. Phys. B111, 441 (1976)

Akushevich I.V., Schumeiko N.M., J. Phys G20, 513 (1994)

Akushevich I.V., Schumeiko N.M., Tolkachev A.E. POLRAD 1.5 (1996), un-

published

Alguard M.J. et al., The E80 Collab., Phys. Rev. Lett. 37, 1258 (1976)

Alguard M.J. et al., The E80 Collab., Phys. Rev. Lett. 37, 1261 (1976)

Alguard M.J. et al., The E80 Collab., Phys. Rev. Lett. 41, 70 (1978)

Alley R. et al., Nucl. Instrum. Methods A365, 1 (1995)

Altarelli G. et al., hep-ph/9701289 (1997)

Altarelli G., Lampe B., Z. Phys. C47, 315 (1990)

Altarelli G., Nason P., Ridolfi G., Phys. Lett. B320, 152 (1994), ERRA-

TUM, Phys. Lett. B325, 538 (1994)

Altarelli G., Parisi G. Nucl. Phys. B126, 298 (1977)

Altarelli G., Ross G., Phys. Lett. B212, 391 (1988)

Anselmino M., Efremov A., Leader E., Phys. Rept. 261, 1 (1995)

Anthony P.L. et al., The E142 Collab., Phys. Rev. Lett. 71, 959 (1993)

Anthony P.L. et al., The E142 Collab., Phys. Rev. D54, 6620 (1996)

Anthony P.L., Szalata Z.M., SLAC-PUB-7201 (1996) 
Apsimon R.J. et al., Nucl. Instr. Meth. A241, 339 (1995)

Arneodo M. et al., Phys. Lett. B364, 107 (1995)

Arnold R., et al., Report SLAC/ESA-4/93 (1993), unpublished

Asham J. et al., The EMC Collab., Phys. Lett. B206, 364 (1988)

Asham J. et al., The EMC Collab., Nucl. Phys. B328, 1 (1990)

Aubert J. et al., Phys. Lett. B123, 275 (1983)

Badelek B. et al., DESY 96-049, hep-ph/9604345 (1996)

Baillon P. et al., Nucl. Instr. Meth. A126, 13 (1975)

Baillon P. et al., Nucl. Instr. Meth. A276, 492 (1989)

Ball R.D., et al., Talk presented at 1996 HERA Workshop (1996), to be published in the proceedings; hep-ph/9609515

Ball R.D., Forte S., Ridolfi G., Nucl. Phys. B444, 287 (1996)

Ball R.D., Forte S., Ridolfi G., Phys. Lett. B378, 255 (1996)

Ball R.D.,De Roeck A., hep-ph/9609309, unpublished

Band H.R. et al., E143 Tech. Note \#110, (1996), unpublished

Band H.R. et al., E154 Tech. Note \#40, (1996), unpublished

Band H.R., AIP Conf. Proc. 343, 245 (1994)

Bardin D.Y., Schumeiko N.M., Nucl. Phys. B127, 242 (1977)

Barnett R.M. et al., Review of Particle Properties, PDG, Phys. Rev. D54, (1996)

Bartels J., Ermolaev B.I., Ryskin M.G., Z. Phys. C70, 1996 (273)

Bartels J., Ermolaev B.I., Ryskin M.G., Z. Phys. C72, 627 (1996)

Bass S.D., Landshoff P.V. Phys.Lett. B336, 537 (1994) 
Baum G. et al., The E130 Collab., Phys. Rev. Lett. 51, 1135 (1983)

Bell J.S., Jackiw R., Nuovo Cim. 60A, 47 (1967)

Bjorken J.D., Phys. Rev. 148, 1467 (1966)

Bjorken J.D., Phys. Rev. 179, 1547 (1969)

Bjorken J.D., Phys. Rev. D1, 1376 (1970)

Bjorken J.D., Paschos E.A., Phys. Rev. 185, 1975 (1969)

Blümlein J., Riemersma S., Vogt A., Nucl. Phys. Proc. Suppl. 51C, 30 (1996)

Blümlein J., Riemersma S., Vogt A., preprint DESY-96-131, (1996)

Blankenbecler R., Brodsky S.J. Phys. Rev. D10, 2973 (1974)

Blankleider B., Woloshyn R.M., Phys. Rev. C29, 538 (1984)

Bloom E.D. et al., Phys. Rev. Lett. 23, 930 (1969)

Bloom E., Gilman F. Phys. Rev. Lett. 25, 1140 (1970)

Bodwin G.T., Qiu J., Phys. Rev. D41, 2755 (1990)

Borel H., et al., IEEE Trans. Nucl. Sci. Vol.42, No.4, 529 (1995)

Borel H. et al., Erratum, IEEE Trans. Nucl. Sci. Vol.42, No.6, 8347 (1995)

Bosted P., et al., E156 SLAC proposal (1997)

Bosted P., E154 Tech. Note \#29, (1995), unpublished

Bourrely C. et al., CPT-96-PE-3327, hep-ph/9604204 (1996)

Bravar A., Talk presented at DIS97, Chicago (1997), to be published in the proceedings

Breidenback M. et al., Phys. Rev. Lett. 23, 935 (1969)

Brodsky S.J., Burkardt M., Schmidt I. Nucl. Phys. B441, 197 (1995) 
Brodsky S.J., Ellis J., Karliner M., Phys. Lett. B206, 309 (1988)

Brodsky S.J. et al., Nucl. Phys. B369, 519 (1992)

Brodsky S.J., Lepage G.P. Proceedings of the 1979 SLAC Summer Institute on Particle Physics, SLAC (1979)

Brodsky S.J., Lu H.J., Phys. Rev. D51, 3652 (1995)

Bunce G. et al., Particle World, Vol.3, 1 (1992)

Burkhardt H., Cottingham W.N., Ann. Phys. 56, 453 (1970)

CERN Application Software Group, CERN Program Library Long Writeup

D506， (1992)

CERN Application Software Group, CERN Program Library Short Writeup

D107, (1992)

CERN Application Software Group, CERN Program Library Short Writeup

C306, (1992)

CERN Courier, V30, No.9, 1 (Dec. 1990) COMPASS proposal, CERN/SPSLC-

96-14 (1996)

Callan C., Gross D., Phys. Rev. Lett. 22, 156 (1969)

Carlitz R.D., Collins J.D., Mueller A.H., Phys. Lett. B214, 229 (1988)

Carlson P.J., Nucl. Instr. Meth. 158, 403 (1979)

Cheng H.- Y., Chin. J. Phys. 35, 25 (1997), hep-ph/9512267

Cheng D.C., O'Neil G.K. Elementary Particle Physics, Addison-Wesley Pub-

lishing Company (1979)

Chupp T.E., et al., Phys. Rev. C36, 2244 (1987)

Ciofi degli Atti C. et al., Phys. Rev. C48, 968 (1993) 
Close F.E., An Introduction to Quarks and Partons, Academic Press (1981)

Close F.E., Roberts R.G., Phys. Lett. B316, 165 (1993)

Close F.E., Roberts R.G. Phys.Lett. B336, 257 (1994)

Collins P.D.B. An Introduction to Regge Theory and High Energy Physics, Cambridge University Press (1977)

Cooper P. et al., Phys. Rev. Lett. 42, 1386 (1979)

Curci G., Furmanski W., Petronzio R., Nucl Phys. B175, 27 (1980)

Dasu S. et al., Phys. Rev. D49, 5641 (1994)

Decker F.-J., et al. SLAC-PUB-7214 (1996)

Dokshitzer Yu.L. Sov. Phys. JETP 46, 461 (1977)

Donnachie A., Landshoff P.V., Phys. Lett. B296, 227 (1992)

Drell S.D., Yan T.-M. Phys. Rev. Lett. 24, 181 (1970)

Dziembowski Z., Martoff C.J., Zyla P., Phys. Rev. D50, 5613 (1994)

Efremov A.V., Teryaev O.V., Phys. Lett. B240, 200 (1990)

Efremov A.V., Teryaev O.V. JINR-E2-88-287 (1988)

Einhorn M.B., Soffer J., Nucl. Phys. B74, 714 (1986)

Ellis J., Jaffe R.L., Phys. Rev. D10, 1663 (1974)

Ellis J., Jaffe R.L., Phys. Rev. D9, 1444 (1974)

Ellis J., Karliner M., Phys. Lett. B213, 73 (1988)

Ellis J., Karliner M. Preprint CERN-TH/95-279, hep-ph/9510402

Engelmann R., et al., Nucl. Instr. Meth. 216, 45 (1983)

Erickson R., et al., SLAC-PUB-7177 (1996)

Farkas, Z.D. et al., SLAC-PUB-1453 (1974) 
Farrar G.R., Jackson D.R. Phys. Rev. Lett. 35, 1416 (1975)

Feynman R.P., Phys. Rev. Lett. 23, 1415 (1969)

Floratos E.G., Kounnas C., Lacaze R., Nucl. Phys. B192, 417 (1981)

Friar J.L. et al., Phys. Rev. C42, 2310 (1990)

Fritzsch H., Gell-Mann M., Proc. XVI Int. Conf. on High Energy Physics, Fermilab (1972), eds. J.D.Jackson, A.Roberts

Fujikawa K., Phys. Rev. Lett. 42, 1195 (1979)

Furmanski W., Petronzio R., Z. Phys. C11, 293 (1982)

Garwin E.L., Tomkiewicz Y., Trines D., Nucl. Instr. Meth. 107, 365 (1973)

Gehrmann T., Stirling W.J., Phys. Rev. D53, 6100 (1996)

Gell-Mann M., Phys. Lett. 8, 214 (1964)

Georgi H., Politzer H.D., Phys. Rev. D9, 416 (1974)

Gluck M. , et al., Phys. Rev. D53, 4775 (1996), hep-ph/9508347

Gluck M., Reya E., Vogt A., Z. Phys. C48, 471 (1990)

Gluck M., Reya E., Vogt A., Z. Phys. C67, 433 (1995)

Gorin A.M., et al., Nucl. Instr. Meth. A251, 461 (1986)

Gorishny S.G., Larin S.A., Phys. Lett. B172, 109 (1986)

Gramblin R.L., Carver T.R., Phys. Rev. A138, 946 (1965)

Graudenz D. et al., Z. Phys. C70, 77 (1996), hep-ph/9506333

Gribov V.N., Lipatov L.N., Sov. J. Nucl. Phys. 15, 438, 675 (1972)

Gross D.J., Wilczek F., Phys. Rev. Lett. 30, 1343 (1973)

Gross D.J., Wilczek F., Phys. Rev. D9, 1974 (980)

Gunion J.F. Phys. Rev. D10, 242 (1974) 
Han M., Nambu Y., Phys. Rev. 139B, 1006 (1965)

Happer W., Rev. Mod. Phys. 44, 169 (1972)

Hayashi T., Hammamatsu Photonics K.K. Application, RES-0791-02

Hayes E.R., et al., Aragone ANL-6916 (1964)

Heimann R.L., Nucl. Phys. B64, 429 (1973)

Hey A.J.G. Proceedings of the 9th Rencontre de Moriond, ed. J.Tran Thanh $\operatorname{Van}(1974)$

t'Hooft G., Veltman M., Nucl. Phys. B44, 189 (1972)

t'Hooft G. Proc. Colloquium on Renormalization of Yang-Mills Fields and Applications to Particle Physics, Marseilles (1972), ed. C.P. Korthals-Altes Hughes $V$. et al., Phys. Lett. B212, 511 (1988)

Ioffe B.L., Khose V.A., Lipatov L.N. Hard Processes, Vol.1, North Holland (1983)

Isgur N., Karl G., Koniuk R., Phys. Rev. Lett. 41, 1269 (1978)

Jackson J.D., Classical Electrodynamics, John Wiley and So., (1975)

Jaffe R.L. hep-ph/9603422

Jaffe R.L., Manohar A., Nucl. Phys. B337, 505 (1990)

Ji X., Phys. Rev. Lett. 78, 610 (1997)

Ji X. hep-ph/9610369

Ji X., Tang J., Hoodbhoy P., Phys. Rev. Lett. 76, 740 (1996)

Kabuss E., Talk presented at DIS97, Chicago (1997), to be published in the proceedings

Kawall D.M., PhD thesis, Stanford University (1995), unpablished 
Kodaira J. et al., Nucl. Phys. B159, 99 (1979)

Kodaira J. et al., Phys. Rev. D20, 627 (1979)

Kodaira J., Nucl. Phys. B165, 129 (1980)

Kolomensky Yu., PhD thesis, University of Massachusetts (1997), unpablished

Kolomensky Yu., E154 collaboration meeting, May 1996, unpublished

Kolomensky Yu., Olson M., Zyla P., E154 Technical Note (1997), unpab-

lished

Kolomensky Yu., Romalis M., E154 Technical Note \#48 (1996), unpablished

Kolomensky Yu., Sabatie F., E154 Technical Note \#44 (1996), unpablished

Kuchto T.V., Schumeiko N.M., Nucl. Phys. B219, 412 (1982)

Kuti J. Internal Spin Structure of the Nucleon, (ed. by V.W.Hughes, C.Cavata)

$134(1995)$

Kuti J. preprint MIT CTP \#234 (1971)

Kuti J., Weisskopf V.F., Phys. Rev. D4, 3418 (1971)

Lacombe M. et al., Phys. Rev. C21, 861 (1980)

Landau L.D., Lifshitz E.M., Quantum Mechanics: non-relativistic theory,

Pergamon Press, (1977)

Landshoff P.V. "Zuoz 1994, Proceedings, Hadronic Aspects of Collider Physics",

135 (1994), hep-ph/9410250

Larin S.A., Vermaseren J.A.M., Phys. Lett. B259, 345 (1991)

Lepage G.P., Brodsky S.J. Phys. Rev. D22, 2157 (1980)

Levchuk L.G., Nucl. Instrum. Methods A345, 496 (1994)

Lung A. et al., Phys. Rev. Lett. 70, 718 (1993) 
Machleid R.R. et al., Phys. Rep. 149, 1 (1987)

Magnon A., Talk presented at DIS97, Chicago (1997), to be published in the proceedings

Mankiewicz L., Phys. Rev. D43, 64 (1991)

Mankiewicz L., Schäfer A., Phys. Lett. B242, 455 (1990)

Martin A.D., Roberts R.G., Stirling W.J., Phys. Lett. B387, 419 (1987)

Maruyama T. et al., SLAC-PUB-92-322 (1992)

Mertig R., van Neerven W.L., Z. Phys. C70, 637 (1996), hep-ph/9506451

(revised version)

Meziani Z.-E., et al., CEBAF proposal (1994)

Middleton H. Ph.D. thesis, Princeton University (1994), unpublished

Mo L.W., Tsai Y.S., Rev. Mod. Phys. 41, 205 (1969)

Møller C., Ann. Phys. (Leipzig) 14, 532 (1932)

Muta T., Foundations of Quantum Chromodynamics, World Scientific (1987)

Namyslowski J.M. in 'Progress in prticle and nuclear physics', Vol.14, ed. by A.Faessler, Pergamon Press, 49 (1995)

Petratos G.G., Report SLAC/ESA-2/93 (1993), unpublished

Politzer H.D., PRL 30, 1346 (1973)

Press W.H., Teukolsky A., Vetterling W.T., Flannery B.P. Numerical Recipies in C, Second edition, Cambridge U.P. (1992)

Pussieux T., Windmolders R., Symposium on the Internal Structure of the Nucleon, p212, World Scientific 1995, ed. by V.W.Hughes, C.Cavata

Rabin N.V., Inst. Exp. Tech. 35, 947 (1992) 
Ratcliffe P., Phys. Lett. B192, 180 (1987)

Ratcliffe P., Phys. Lett. B192, 309 (1987)

Reya E., Schladming lectures 1993, Springer Lecture Majorana Summer School of Subnuclear Physics, Erice 1989, Plenum Press 1990 [CERN-TH.5675/90];

Reya E. Proc. of the HERA-Workshop, Hamburg 1991, eds. Buchmüller W. and G. Ingelman, DESY, 1992, vol. I, p.379.

Reya E., Schladming lectures 1993, Springer Lecture Notes in Physics 426, $175(1994)$

Roeck De A., Talk presented at DIS97, Chicago (1997), to be published in the proceedings

Romalis M.V., E154 Tech. Note \#34, (1996), unpublished

Romalis M.V., PhD thesis, Princeton University (1997), unpablished

Romalis M.V., Smith T.B., E154 Target Polarization Tech. Note, (1996), unpublished

Schüler P., Talk presented at DIS97, Chicago (1997), to be published in the proceedings

Scott G.G., Sturner H.W., Phys. Rev. 184, 184 (1969)

Sokolov A.A., Ternov I.M., Sov. Phys. Doklady 8, 1203 (1964)

Sorrell L., E154 Tech. Note \#25, (1995), unpublished

Stuart L., E154 Tech. Note \#6, (1995), unpublished

Swartz M., et al., Nucl. Instrum. Methods A363, 526 (1995)

Tomkiewicz Y., Garwin E.L., SLAC-PUB-1356 (1973)

Tsai Y.S., SLAC-PUB-884 (1971) 
Vogelsang W., Z. Phys. C50, 275 (1991)

Vogelsang W., Phys. Rev. D54, 2023 (1996)

Wandzura S., Wilczek F., Phys. Lett. 72B, 195 (1977)

Wang X.J., E154 collaboration meeting, May 1996, unpublished

Weinberg S., Phys. Rev. 150, 1313 (1966)

Whitlow L.W. et al., Phys. Lett. B250, 193 (1990)

Whitlow L.W., SLAC-PUB-0357 (1990)

Wilson K., Phys. Rev. 179, 1499 (1969)

Woods M. SLAC-PUB-7320 (1996)

$X u J ., \mathrm{PhD}$ thesis, Syracuse University (1994), unpablished

Yang C.N., Mills R.L., Phys. Rev. 96, 191 (1954)

Young C., E154 Tech. Note \#35, (1996), unpublished

Zuilhof M.J., Tjon J.A. Phys. Rev. C22, 2369 (1980)

Zweig G., CERN-TH-401 (1964)

Zweig G., CERN-TH-412 (1964)

Zyla P., E154 Tech. Note \#24, (1995), unpublished

Zyla P., Averett T.D., E154 Tech. Note \#43, (1996), unpublished 


\section{APPENDIX A}

\section{DILUTION FACTOR}

In extracting neutron spin structure functions from the experimental data, one needs to know what fraction of the detected electrons scatter off polarized neutrons within the target, referred to as the dilution factor. An important component of the answer is the number of events generated by scattering off ${ }^{3} \mathrm{He}$ compared to the entire target, i.e. the ${ }^{3} \mathrm{He}$ dilution factor

$$
f=\frac{\text { number of } e^{-} \text {scattered off }{ }^{3} \mathrm{He}}{\text { number of } e^{-} \text {scattered off the entire target }} .
$$

Knowing the target parameters documented in Reference [87] one can model the dilution factor. A second method uses runs during the experiment with a variable content of ${ }^{3} \mathrm{He}$ and yields a direct measurement. Here we summarize the systematic studies of the dilution factor for all targets used in the experiment [178]. 


\section{A.1 Modeling dilution factor}

Let's divide the target into three major parts: glass, Nitrogen and Helium-3. Using $N_{i}$ as the number of electrons scattered off the i-th component $(i=$ $H e, N_{2}, g l$ for Helium, Nitrogen and glass respectively), we rewrite equation (A.1) as

$$
f=\frac{N_{H e}}{N_{H e}+N_{N_{2}}+N_{g l}} .
$$

More precisely $N_{i}$ is the number of electrons in a given $x$ bin and thus is a function of the average Bjorken $\bar{x}$ and the average momentum transfer $\bar{Q}^{2}$ for that $\operatorname{bin}^{1}, N_{i}=N_{i}\left(\bar{x}, \bar{Q}^{2}\right)$. Denoting the number of incident particles by $I$ and the cross-sectional area of the beam by $S$, we can form the number of incident particles per unit area as $I / S$. Let the thickness of a scattering material be $L_{i}$ and the number density $n_{i}$. The number of scattering centers is $L_{i} S n_{i}$. If the scattering cross section is $\sigma_{i}$ and the spectrometer acceptance equals $a$, the number of scattered electrons is

$$
N_{i}\left(\bar{x}, \bar{Q}^{2}\right)=\left(\frac{I}{S}\right)\left(L_{i} S n_{i}\right) \int_{D_{[x b i n]}} d \Omega\left(x, Q^{2}\right) \sigma_{i}\left(x, Q^{2}\right) a\left(x, Q^{2}\right),
$$

where $D_{[x \text { bin }]}$ is the angular integration region for a given $x$ bin and $d \Omega\left(x, Q^{2}\right)$ is the associated measure. The spectrometer acceptance $a\left(x, Q^{2}\right)$ varies slowly within every $x$ bin except for the lowest one, for which the cross section $\sigma_{i}\left(x, Q^{2}\right)$ changes mildly. Thus the integration yields

$$
\int_{D_{[x b i n]}} d \Omega\left(x, Q^{2}\right) \sigma_{i}\left(x, Q^{2}\right) a\left(x, Q^{2}\right) \approx \sigma_{i}\left(\bar{x}, \bar{Q}^{2}\right) a\left(\bar{x}, \bar{Q}^{2}\right) .
$$

\footnotetext{
${ }^{1}$ Please keep in mind that $\bar{x}$ and $\bar{Q}^{2}$ are not independent quantities in our experiment. They are related via the fixed spectrometer angle.
} 
The scattering cross section $\sigma_{i}\left(x, Q^{2}\right)$ for an element of the atomic number $A_{i}$ with $Z_{i}$ protons and $A_{i}-Z_{i}$ neutrons can be constructed from the cross sections of protons and neutrons,

$$
\sigma_{i}\left(x, Q^{2}\right)=K\left(x, Q^{2}\right)\left(Z_{i} F_{2}^{p}\left(x, Q^{2}\right)+\left(A_{i}-Z_{i}\right) F_{2}^{n}\left(x, Q^{2}\right)\right) E M C\left(A_{i}, x\right),
$$

where $K\left(x, Q^{2}\right)$ contains kinematic factors and $\operatorname{EMC}(A, x)$ is the EMC effect [29], which ensures the proper treatment of the nucleon binding effects. Substituting equations (A.3-A.5) into equation (A.2) and canceling out common factors, we obtain a useful formula

$$
f\left(\bar{x}, \bar{Q}^{2}\right)=\frac{R_{H e}\left(\bar{x}, \bar{Q}^{2}\right)}{R_{H e}\left(\bar{x}, \bar{Q}^{2}\right)+R_{N_{2}}\left(\bar{x}, \bar{Q}^{2}\right)+R_{g l}\left(\bar{x}, \bar{Q}^{2}\right)}
$$

with

$$
R_{i}\left(\bar{x}, \bar{Q}^{2}\right)=\left(Z_{i} F_{2}^{p}\left(\bar{x}, \bar{Q}^{2}\right)+\left(A_{i}-Z_{i}\right) F_{2}^{n}\left(\bar{x}, \bar{Q}^{2}\right)\right) E M C\left(A_{i}, \bar{x}\right) L_{i} n_{i}
$$

Note that the beam intensity $I$ and the acceptance $a$ have canceled out. To compare this modeled $f$ with the experiment, we need additional corrections to formula (A.6).

\section{A.1.1 Radiative corrections}

The cross section $\sigma_{i}$ in eq. (A.5) comes from the leading order calculation of the scattering at the target. We can include higher order contributions in terms of the internal radiative corrections $r c_{i}^{i n t}$. Also an electron traversing the target looses its energy mainly due to bremsstrahlung. This can be accounted for by the external radiative corrections $r c_{i}^{e x t}$. The total effect is 
a convolution of both corrections symbolically written as $r c_{i}=r c_{i}^{e x t} \otimes r c_{i}^{i n t}$. When the electron scatters off the upstream glass window, it must travel through the sidewall of the target cell to be detected in the spectrometer. In the case of scattering off the downstream window the electron enters the spectrometer without traversing any additional target material. We thus divide the glass contribution $R_{g l}$ into upstream $R_{g l}^{u p}$ and downstream $R_{g l}^{\text {down }}$ contributions and rewrite the formula (A.6) for $f$ as ${ }^{2}$

$$
f=\frac{R_{H e} r c_{H e}}{R_{H e} r c_{H e}+R_{N_{2}} r c_{N_{2}}+R_{g l}^{\text {down }} r c_{g l}^{\text {int }}+R_{g l}^{\text {up }} r c_{g l}},
$$

where $r c_{g l}^{i n t}$ is the internal radiative correction for the glass and $r c_{g l}$ is the total correction as defined before.

\section{A.1.2 Helium contribution}

The ${ }^{3}$ He nucleus consists of $Z=2$ protons and $A-Z=1$ neutron. The thickness of ${ }^{3} \mathrm{He}, L_{H e}$, is the internal length of the target (roughly $30 \mathrm{~cm}$, see Ref. [87]). The density $n_{H e}\left(\approx 10\right.$ amagats $\left.^{3}\right)$ can be converted to mol/cc via the constant $4.46 \times 10^{-5}(\mathrm{~mol} / \mathrm{cc})$ / amagat .

\section{A.1.3 Nitrogen contribution}

A molecule of nitrogen consist of two atoms. Each nucleus contains $Z=7$ protons and $A-Z=7$ neutrons. The thickness $L_{N_{2}}$ is the same as for ${ }^{3} \mathrm{He}$,

\footnotetext{
${ }^{2}$ There is an implicit $\left(\bar{x}, \bar{Q}^{2}\right)$ dependence of all the quantities.

${ }^{3}$ See reference [87] for all target parameters. Any non-referenced target quantity shall be looked up in this source.
} 
namely it is equal to the target's length. The $N_{2}$ density is on the order of 0.088 amagats $\left(3.9 \times 10^{-6} \mathrm{~mol} / \mathrm{cc}\right.$ at STP $)$.

\section{A.1.4 Glass compounds}

The glass number density $n_{g l}$ requires a bit more attention. First we split it into separate elements. Say, for example, we want to know the content $n_{B}$ of boron. Let's take one $c c$ of glass which weighs $2.52 \mathrm{~g}$. It contains $5 \%$ boron oxide $\mathrm{B}_{2} \mathrm{O}_{3}$, i.e. $0.126 \mathrm{~g}$. The nucleus of oxygen is built from 8 protons and 8 neutrons. So one mole weighs $8+8=16 \mathrm{~g}$. Boron (5 protons) exists in nature in a form of two isotopes [179], $80 \%$ with 6 neutrons and the remaining $20 \%$ with 5 neutrons. This gives 5.8 neutrons on the average and implies 10.8 grams/mole for boron. Thus one mole of $\mathrm{B}_{2} \mathrm{O}_{3}$ has a molar mass $2 * 10.8+3 * 16=69.6 \mathrm{~g} / \mathrm{mol}$. A mass of 0.126 grams of boron oxide contains $0.126 / 69.6=0.00181$ moles of $B_{2} O_{3}$ molecules and twice as many atoms of boron $B$. Thus $n_{B}=3.62 \times 10^{-3} \mathrm{~mol} / \mathrm{cc}$. In a similar manner we compute densities of other glass compounds. The results are summarized in table A.1.

Table A.1: Glass Compounds Density $n$ (mol/cc).

\begin{tabular}{lrrrrrrr}
\hline & $\mathrm{O}$ & $\mathrm{Si}$ & $\mathrm{Al}$ & $\mathrm{Mg}$ & $\mathrm{Ca}$ & $\mathrm{B}$ & $\mathrm{Na}$ \\
\hline $\mathrm{Z}$ & 8 & 14 & 13 & 12 & 20 & 5 & 11 \\
$\mathrm{~A}-\mathrm{Z}$ & 8 & $92 \% 14$ & 14 & $79 \% 12$ & $97 \% 20$ & $80 \%$ & 12 \\
& & $5 \% 15$ & & $10 \% 13$ & $1 \% 22$ & $20 \% 5$ & \\
& & $3 \% 16$ & & $11 \% 14$ & $2 \% 24$ & & \\
$n \times 10^{3}$ & 78.2 & 25.1 & 8.87 & 5.62 & 3.15 & 3.62 & 0.81 \\
\hline
\end{tabular}


Now we can calculate the rates $R_{i}$ for the upstream $\left(L_{g l}^{u p} \sim 60 \mu m\right)$ and downstream $\left(L_{g l}^{\text {down }} \sim 60 \mu m\right)$ windows for each element from formula (A.7) and sum them up to get the total glass contribution.

\section{A.1.5 Numerical results}

We use the proton $F_{2}^{p}$ and the deuteron $F_{2}^{d}$ structure functions as measured by NMC [124] and calculate the neutron structure function $F_{2}^{n}=$ $2 * F_{2}^{d}-F_{2}^{p}$. For the EMC effect we use Smirnov [180] parametrization. The radiative corrections were generated using POLRAD Version 1.5 [118]. A typical $f$ result can be seen in figure (A.1). We also show the impact of the radiative corrections.

On the average $56 \%$ of the events come from scattering off helium-3, $4 \%$ off nitrogen and the remaining $40 \%$ off glass ( $19 \%$ from the upstream window and $21 \%$ from the downstream one).

\section{A.1.6 Error analysis}

Two categories of quantities contribute to the uncertainty in the dilution $f$.

The first contains quantities whose relative errors are $x$ independent: the thickness of the cell windows, $L_{g l}^{u p}$ and $L_{g l}^{\text {down }}$, the length of the target, $L_{H e}=L_{N_{2}}$, the density of Helium, $n_{H e}$, Nitrogen, $n_{N_{2}}$, and the glass, $n_{g l}$, the isotopes percentages, the glass compound uncertainties and the beam halo.

The second category includes quantities whose relative errors are functions of x, namely the structure functions $F_{2}^{p}$ and $F_{2}^{d}$, the EMC effect and 


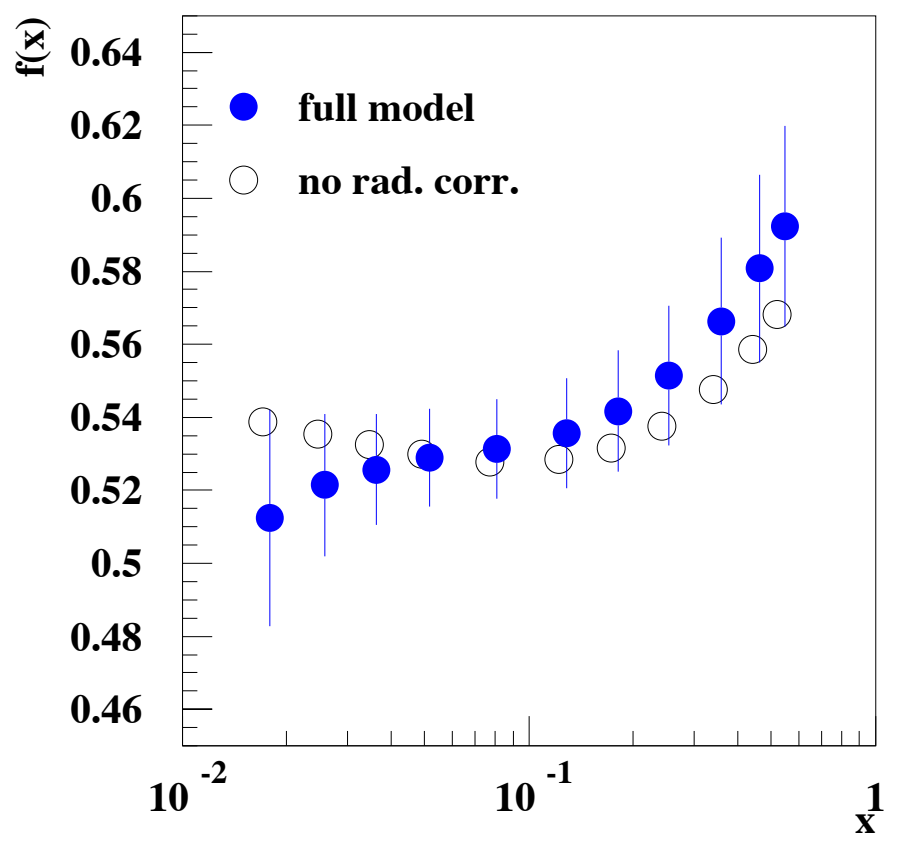

Figure A.1: Typical $f$ model result. Target 'Picard', 2.75'.

the radiative corrections, $r c_{i}$. We introduce other dilution factor definitions (see eq. A.8), for nitrogen,

$$
f_{N}=\frac{R_{N_{2}} r c_{N_{2}}}{R_{H e} r c_{H e}+R_{N_{2}} r c_{N_{2}}+R_{g l}^{\text {down }} r c_{g l}^{\text {int }}+R_{g l}^{u p} r c_{g l}}
$$

and glass,

$$
f_{G}^{u p}=\frac{R_{g l}^{u p} r c_{g l}}{R_{H e} r c_{H e}+R_{N_{2}} r c_{N_{2}}+R_{g l}^{\text {down }} r c_{g l}^{\text {int }}+R_{g l}^{u p} r c_{g l}},
$$

and similarly $f_{G}^{\text {down }}$. Note that $f+f_{N}+f_{G}^{u p}+f_{G}^{\text {down }}=1$. 


\section{Relative $x$ independent uncertainties}

Using the dilution factor formulas (A.7) and (A.8), we can calculate the $x$ independent errors. The individual contributions from the nitrogen related quantities $\left(n_{N_{2}}\right)$, call them $N$ with uncertainty $\delta N$, are of the form

$$
\left(\frac{\delta f}{f}\right)_{N}=f_{N} \frac{\delta N}{N} .
$$

Similarly for the glass $G \pm \delta G$ contributions $\left(L_{g l}^{u p}, L_{g l}^{\text {down }}\right)$,

$$
\left(\frac{\delta f}{f}\right)_{G^{\text {up } / \text { down }}}=f_{G}^{\text {up } / \text { down }} \frac{\delta G^{\text {up } / \text { down }}}{G^{\text {up } / \text { down }}},
$$

and for $n_{g l}$

$$
\left(\frac{\delta f}{f}\right)_{G}=f_{G} \frac{\delta G}{G}
$$

with $f_{G}=f_{G}^{u p}+f_{G}^{\text {down }}$. And for the helium $H \pm \delta H$ related,

$$
\left(\frac{\delta f}{f}\right)_{H}=(1-f) \frac{\delta H}{H} .
$$

The propagation of the target length error from $L_{H e}=L_{N_{2}}(\equiv L \pm \delta L)$ can be obtained from the above formulas as $(\delta f / f)_{L}=(\delta f / f)_{N=L}+(\delta f / f)_{H=L}$ i.e.

$$
\left(\frac{\delta f}{f}\right)_{L}=\left(1-f-f_{N}\right) \frac{\delta L}{L} .
$$

The halo effect on the central value of the dilution factor can be obtained from the halo target run 1093 as compared to full reference cell run 1094. The measured ratio of rates $7 \times 10^{-4}$ indicates that it is negligible. After that measurement there were many beam tune changes, but no halo target runs were performed. To extend the halo result to all runs, let us take the rates 
of the no target run 1090 (which were 5 times smaller than the halo rates) into account and assume that the ratio of the halo to the no target rates does not change much. Consistently those no target vs. target rates stayed on a $10^{-4}$ level throughout the experiment indicating (if our assumption is correct) that the halo rates were $10^{-3}$ times smaller than the target rates, and thus negligible. However, knowing that this is based on an ad hoc assumption we try to estimate the confidence based on the rate stability. Assume that the effect of the halo can be bounded by $\sqrt{\text { variance }} /$ mean $(\approx 1 \%)$ of the target rate distribution. This value will be taken as the uncertainty of the dilution due to the halo. The errors resulting from the limited knowledge of the isotopic percentage distribution and glass compounds have been estimated to contribute $0.01 \%$ to the uncertainty in $f$, thus negligible.

The above considerations are summarized in table (A.2) for the average

Table A.2: Propagation of $x$ Independent Relative Errors.

\begin{tabular}{|c|c|c|c|c|c|c|c|c|c|}
\hline$q$ & $L$ & $n_{N}$ & $n_{H e}$ & $L_{g l}^{u p}$ & $L_{g l}^{\text {down }}$ & $n_{g l}$ & iso & $g l^{\mathrm{comp}}$ & halo \\
\hline$\frac{\delta q}{q}(\%)$ & 0.5 & 1.5 & 1.5 & 3 or 5 & 3 or 5 & 0.5 & 0.1 & see $[87]$ & \\
\hline$\left(\frac{\delta f}{f}\right)_{q}(\%)$ & 0.20 & 0.06 & 0.66 & $.57 / .95$ & $.63 / 1.1$ & 0.20 & negl & negl & 1.0 \\
\hline
\end{tabular}

results $\left(f=56 \%, f_{N}=4 \%, f_{G}^{u p}=19 \%\right.$ and $\left.f_{G}^{\text {down }}=21 \%\right)$.

\section{The $x$ dependent uncertainties}

We can deal with the remaining quantities in a similar manner. The EMC factor can be readily put through equations (A.11), (A.13) and (A.14) for each element. The largest effect is for helium, oxygen and silicon. We 
will treat the errors for all the elements as uncorrelated and add them in quadrature to obtain the total uncertainty. As for $F_{2}^{p}$ and $F_{2}^{d}$ let us first rewrite equation (A.7) in terms of those quantities,

$$
R_{i}=\left(\left(2 Z_{i}-A_{i}\right) F_{2}^{p}+2\left(A_{i}-Z_{i}\right) F_{2}^{d}\right) E M C_{i} L_{i} n_{i}
$$

Now we use a formula similar to eq. (A.15) to propagate the errors:

$$
\left(\frac{\delta f}{f}\right)_{j}=\left((1-f) a_{H}^{j}-f_{N} a_{N}^{j}-f_{G} a_{G}^{j}\right) \frac{\delta F_{2}^{j}}{F_{2}^{j}} .
$$

where

$$
a_{i}^{p}=\frac{2 Z_{i}-A_{i}}{\left(2 Z_{i}-A_{i}\right)+2\left(A_{i}-Z_{i}\right) F_{2}^{d} / F_{2}^{p}}
$$

and for deuteron

$$
a_{i}^{d}=\frac{2\left(A_{i}-Z_{i}\right)}{\left(2 Z_{i}-A_{i}\right) F_{2}^{p} / F_{2}^{d}+2\left(A_{i}-Z_{i}\right)} .
$$

The radiative corrections will be treated somewhat differently. We will take the full effect of the correction as the error, i.e. the error is the difference between $f$ calculated with radiative corrections and without them, $\delta f_{r c}=$ $\left|f_{r c}-f_{\text {norc }}\right|$. Analytically, to the first order, it is given by

$$
\left(\frac{\delta f}{f}\right)_{r c}=(1-f) \delta r c_{H e}-f_{N} \delta r c_{N}-f_{g l}^{\text {down }} \delta r c_{g l}^{i n t}-f_{g l}^{u p} \delta r c_{g l} .
$$

The numerical results of this section are quoted in table A.3 for the target 'Picard', $2.75^{\circ}$ spectrometer.

\section{A.1.7 Summary}

One can see that the largest contribution to the error comes from the radiative corrections except for a few intermediate $x$ points where errors 
Table A.3: Propagation of $x$ Dependent Relative Errors, Quoted in \%. The Average $\bar{Q}^{2}$ is in the Units of $\mathrm{GeV}^{2}$.

\begin{tabular}{llllccccc}
\hline $\bar{x}$ & $\bar{Q}^{2}\left(\frac{\delta E M C}{E M C}\right)_{H e}\left(\frac{\delta E M C}{E M C}\right)_{O}$ & $\left(\frac{\delta F_{2}^{p}}{F_{2}^{p}}\right)$ & $\left(\frac{\delta F_{2}^{d}}{F_{2}^{d}}\right)\left(\frac{\delta f}{f}\right)_{E M C}$ & $\left(\frac{\delta f}{f}\right)_{F_{2}}$ & $\left(\frac{\delta f}{f}\right)_{r c}$ \\
\hline 0.017 & 1.1 & 0.47 & 1.4 & 2.6 & 2.1 & 0.36 & 1.3 & 5.1 \\
0.024 & 1.5 & 0.45 & 1.3 & 2.4 & 2.1 & 0.33 & 1.2 & 2.7 \\
0.034 & 2.0 & 0.42 & 1.2 & 2.2 & 2.1 & 0.30 & 1.1 & 1.3 \\
0.049 & 2.5 & 0.40 & 1.1 & 2.1 & 2.0 & 0.28 & 1.0 & 0.15 \\
0.077 & 3.3 & 0.37 & 1.0 & 2.0 & 1.9 & 0.26 & 0.98 & 0.66 \\
0.12 & 4.1 & 0.36 & 0.94 & 2.2 & 1.7 & 0.24 & 0.97 & 1.4 \\
0.17 & 4.6 & 0.35 & 0.94 & 2.3 & 1.8 & 0.24 & 1.0 & 1.9 \\
0.24 & 5.1 & 0.37 & 1.0 & 2.3 & 1.9 & 0.25 & 1.0 & 2.5 \\
0.34 & 5.5 & 0.41 & 1.2 & 2.3 & 2.1 & 0.28 & 0.99 & 3.3 \\
0.44 & 5.8 & 0.47 & 1.5 & 2.2 & 2.1 & 0.33 & 0.94 & 3.8 \\
0.52 & 6.1 & 0.53 & 1.8 & 2.2 & 2.0 & 0.38 & 0.87 & 4.1 \\
\hline
\end{tabular}

from $L_{g l}, F_{2}$ and halo are of the same order. To obtain the total uncertainty, one has to add all the single uncertainties in quadrature. The results are given in table A.4 (see also figure A.1).

Table A.4: Model Results for the Target 'Picard', 2.75'.

\begin{tabular}{cccc}
\hline $\bar{x}$ & $f$ & $\delta f$ & $\left(\frac{\delta f}{f}\right)(\%)$ \\
\hline 0.017 & 0.512 & 0.029 & 5.8 \\
0.024 & 0.521 & 0.019 & 3.7 \\
0.034 & 0.526 & 0.015 & 2.8 \\
0.049 & 0.529 & 0.013 & 2.4 \\
0.077 & 0.531 & 0.013 & 2.5 \\
0.12 & 0.536 & 0.015 & 2.7 \\
0.17 & 0.542 & 0.016 & 3.0 \\
0.24 & 0.551 & 0.019 & 3.4 \\
0.34 & 0.566 & 0.023 & 4.0 \\
0.44 & 0.581 & 0.025 & 4.4 \\
0.52 & 0.592 & 0.027 & 4.6 \\
\hline
\end{tabular}




\section{A.2 Measurement}

The basic definition of the dilution factor in eq. (A.1) suggests a method of measurement. Let a rate $R t$ be the normalized number of scattered electrons. Denote by $R t^{t g}$ the rate from the full target and by $R t_{H e}^{t g}$ the rate from the helium contained in that target. These are exactly the quantities present in the definition

$$
f=\frac{R t_{H e}^{t g}}{R t^{t g}}
$$

The rates in the denominator are measured directly in the experiment. To obtain the rates from helium only, we put a target without helium into the beam $\left(R t_{\text {empty }}^{t g}\right)$. Taking the difference between rates from full and empty targets, one arrives at the helium rates,

$$
f=\frac{R t^{t g}-R t_{e m p t y}^{t g}}{R t^{t g}}
$$

\section{A.2.1 Experimental procedure}

We do not empty the target to measure $R t_{\text {empty }}^{t g}$ rates. Instead we establish the rates from helium via runs with a reference cell filled with a variable pressure of ${ }^{3} H e$. Suppose we obtain the rate $R t_{1}$ from a run with the pressure $P_{1}$ and the rate $R t_{2}$ from a run with the pressure $P_{2}$. We now compute the rate from helium per unit of pressure, per unit of length:

$$
\text { slope }=\frac{R t_{1}-R t_{2}}{P_{1}-P_{2}}
$$

and

$$
\frac{\text { Rate of }{ }^{3} \mathrm{He}}{\text { Press } * \text { Length }}=\frac{\text { slope }}{L_{\text {ref }}}
$$


where $L_{r e f}$ is the length of the reference cell. If the target is filled with pressure $\rho_{t g}$ of helium and has a length $L_{t g}$, then the rate from the target helium can be obtained by multiplying the last equation by $\rho_{t g}$ and $L_{t g}$. Thus the dilution $f$ can be measured as

$$
f=\left(\frac{R t_{1}-R t_{2}}{R t_{t g}}\right)\left(\frac{\rho_{t g}}{P_{1}-P_{2}}\right)\left(\frac{L_{t g}}{L_{r e f}}\right),
$$

or equivalently

$$
f=\text { slope }\left(\frac{\rho_{t g}}{R t_{t g}}\right)\left(\frac{L_{t g}}{L_{r e f}}\right) .
$$

The pressure of helium in the reference cell was measured in psia at an operating temperature $T_{r e f}$ while the pressure in the target $\rho_{t g}$ is given in amagats (standard temperature). We thus need to convert psia at $T_{\text {ref }}$ to amagats,

$$
\rho_{i}=P_{i}\left(\frac{C a l}{T_{r e f}}\right)
$$

where $C a l=18.6$ amagat $* K / p$ sia. We can still use the formula (A.26) with a redefined slope $=\left(R t_{1}-R t_{2}\right) /\left(\rho_{1}-\rho_{2}\right)$.

\section{A.2.2 Rate dependence correction}

More work is needed to extract the experimental result properly. The rates $R t$ that are used in calculation from measurement are "full" rates. We need to convert them to "measured" rates $R m$ by taking into account the efficiency $\varepsilon_{f}$ of our detectors. That efficiency can be factorized onto a constant part $\varepsilon$ and a rate dependent piece characterized by coefficient $\alpha$ defined by the equation $\varepsilon_{f}(R t)=\varepsilon\left(1-\alpha \frac{R t}{R t^{t g}}\right)$. The "measured" and "full" 
rates are related to each other by

$$
R m=R t \varepsilon\left(1-\alpha \frac{R t}{R t^{t g}}\right) .
$$

One can say that we have assumed linearity of efficiency as a function of rate or, equivalently, that the above equation is written to the first order in $\alpha$. To first order we can rewrite the definition of $f$, eq. (A.22), as

$$
f=1-\left(\frac{R m_{e m p t y}^{t g}}{R m^{t g}}\right)\left(\frac{1-\alpha}{1-\alpha \frac{R m_{e m p t y}^{t g}}{R m^{t g}}}\right) \approx f_{m}\left(1+\alpha \frac{R m_{e m p t y}^{t g}}{R m^{t g}}\right),
$$

where $f_{m}$ is defined like $f$ with the rates $R t$ replaced by measured $R m$. In our

experiment the ratio of rates $R m_{\text {empty }}^{t g} / R m^{t g}$ was roughly 0.5 . The expected correction to $f_{m}$ is thus $\frac{\alpha}{2} f_{m}$.

Technically this correction is implemented on a run by run basis. That is, the rate $R m$ from a single run is multiplied by the rate dependent factor of efficiency, $\left(1+\alpha R m / R m^{t g}\right)$, and then used in further calculations.

The coefficient $\alpha$, measured for each target via the "pulse fiction" method (cf. section 3.7.1, page 132) is a function of $x$ and ranges from few a to several percent (with an error much smaller than $\alpha$ itself). Actually the method puts an upper bound on the rate dependence. We thus adopt the following philosophy: We correct the dilution by half of the overestimated rate dependence and take the other half as the error due to it. An example of the correction can be read from table A.6.

\section{A.2.3 Background subtraction}

One should note that our electrons are not a pure sample. We have some misidentified pions $\left(C_{\pi}\right.$ fraction of rate) and electrons $\left(C_{e}\right.$ fraction, called 
positron contamination in our jargon) coming from charge symmetric processes (pair production) rather than DIS. Let us again denote the measured rates by $R m$ and the corrected rates by $R t$, and by $\beta$ the rate dependent part. That allows us to relate the rates as follows:

$$
R m=R t\left(1+\beta\left(C_{e}+C_{\pi}\right)\right)
$$

with $\beta=1$ for full target, and to compute dilution

$$
f=1-\left(\frac{R m_{e m p t y}^{t g}}{R m^{t g}}\right)\left(\frac{1+\beta C_{e+\pi}}{1+\beta_{e m p t y} C_{e+\pi}}\right) \approx f_{m}+\left(1-f_{m}\right)\left(\beta_{e m p t y}-\beta\right) C_{e+\pi}
$$

where $C_{e+\pi}=C_{e}+C_{\pi}$. To get some feeling for the importance of this correction, we suppose that $1-f_{m} \approx f_{m}$ and $\beta_{\text {empty }}=1.2$ for all $x$, so $f=f_{m}\left(1+0.2 C_{e+\pi}\right)$, i.e. the relative correction to $f_{m}$ is about $20 \%$ of the total contamination fraction.

The pion and positron contaminations for full target runs, measured for our electron definition (cf. section 3.6.1, page 125) are rapidly decreasing to zero functions of $x$ with the largest sum $C_{e+\pi}$ of about 0.2 at the lowest $x$. As mentioned above the contamination for an empty cell has been found to be 1.2 times larger than for the full cell and constant with $x$.

In practice the correction is implemented for every run in accordance with eq. (A.30). It is known for full and empty targets. To apply it to an arbitrary ${ }^{3} \mathrm{He}$ pressured cell we interpolate linearly between those two points. Furthermore the empty cell measurement suffers from low statistics. We thus assign an error equal to the correction itself. 


\section{A.2.4 Run selection}

There are two technically different approaches to the calculation of the slope (eq. A.23) from the reference cell runs. The first takes into account adjacent runs with different pressures $P$ from which one calculates the slope. Then the slopes from all coupled runs within considered target are averaged. The advantage of this possibility is the smaller sensitivity of the slope to the spectrometers or beam changes with time. The second considers all available runs and fits a straight line through $R t(P)$ points. The slope of that line is exactly the slope we use in computations. The advantage of this approach is better statistics, because there are many reference cell runs that are not accompanied by a second run at a different pressure. Furthermore, the majority of the single runs are during the polarized target runs, while couples are mainly before and after those. We thus have a better average of changing conditions in this case.

We proceed with the second approach and have checked that both give the same results within the errors.

By all reference runs we mean those that pass our selection criteria. To be considered a good run, we check that there were no hardware problems, that the spectrometer setup and the beam tune and current were the same as for the polarized target runs. We also eliminate the runs that are far before or far after the polarized target runs.

The target rates are established from all the polarized data that are considered good for asymmetry analysis (cf. section 3.4.1, page 117), except the 
target polarization cut, which is not required here. A typical result for the dilution factor is shown in figure (A.2).

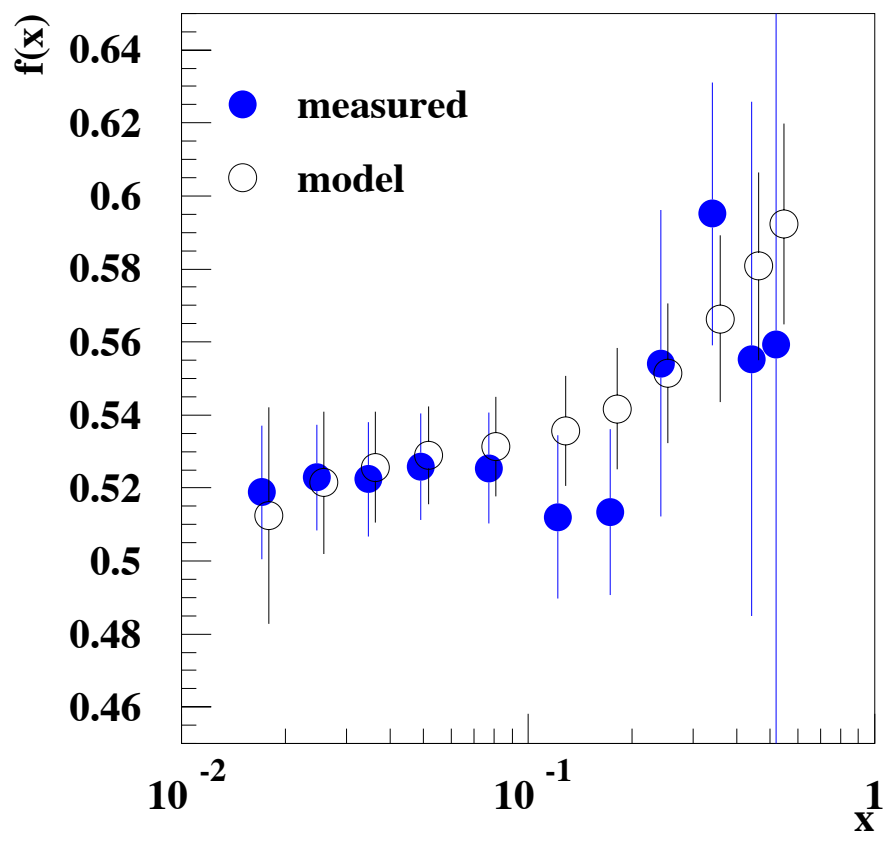

Figure A.2: Measured result for target 'Picard', $2.75^{\circ}$.

\section{A.2.5 Error analysis}

To analyze error propagation, let us first look at $f$ given by the formula (A.26). We immediately see that the quantities like $L_{t g}, L_{r e f}, \rho_{t g}, R t_{t g}$ have the following influence on the dilution factor error:

$$
\left(\frac{\delta f}{f}\right)_{q}=\frac{\delta q}{q}
$$


All other errors contribute via the slope. Note that the slope, which comes from a linear fit to $(R t, P)$ pairs, does not take into account the error in the pressure $P$. We need to estimate it separately. Many of the reference cell runs were made without any helium. Then the pressure, say $P_{2}$ in equation (A.25), equals zero with high precision, and the dilution $f \sim 1 / P_{1}$. The pressure $P_{1}$ is normalized to standard temperature via formula (A.27). Thus eq. (A.32) can also be used for the pressure $P$ and the temperature $T$. We can now set up table A.5 of errors.

Table A.5: Relative $x$ Independent Errors For Measured $f$.

\begin{tabular}{lccccc}
\hline$q$ & $L_{t g}$ & $L_{r e f}$ & $\rho_{t g}$ & $P_{\text {ref }}$ & $T_{\text {ref }}$ \\
\hline$\left(\frac{\delta q}{q}=\left(\frac{\delta f}{f}\right)_{q}\right)(\%)$ & 0.5 & 0.5 & 1.5 & 0.5 & 0.9 \\
\hline
\end{tabular}

The rates from the target, $R t_{t g}$, are limited by statistics. Also statistical in nature are errors in the reference cell rates, $R t_{r e f}$. The latter, as mentioned before, are the only rates that produce the error in the slope i.e. $(\delta f / f)\left(R t_{\text {ref }}\right)=(\delta$ slope/slope $)$. In addition we have the full rate dependence correction, $(\delta f / f)_{\text {rate }}$, and the full background subtraction, $(\delta f / f)_{b g}$, errors. All of these errors are summarized in table A.6 for target 'Picard', $2.75^{\circ}$. Also the result for $f$ is given there. One can see that the dominant errors are statistical. 
Table A.6: Measured $(\delta f / f)(x)$ Errors in \% and $f$ Results for 'Picard', $2.75^{\circ}$.

\begin{tabular}{lcrlllll}
\hline $\bar{x}$ & $\left(\frac{\delta f}{f}\right)_{R t^{t g}}$ & $\left(\frac{\delta f}{f}\right)_{R t^{\text {ref }}}$ & $\left(\frac{\delta f}{f}\right)_{\text {rate }}$ & $\left(\frac{\delta f}{f}\right)_{b g}$ & $f$ & $\delta f$ & $\frac{\delta f}{f}$ \\
\hline 0.017 & 1.1 & 2.8 & 1.6 & 2.9 & 0.52 & .025 & 4.9 \\
0.024 & 0.98 & 1.8 & 1.7 & 1.2 & 0.52 & .018 & 3.5 \\
0.034 & 1.1 & 2.1 & 1.7 & 0.44 & 0.52 & .018 & 3.5 \\
0.049 & 0.93 & 1.8 & 1.5 & 0.16 & 0.53 & .017 & 3.2 \\
0.077 & 0.95 & 2.0 & 1.4 & 0.05 & 0.53 & .017 & 3.3 \\
0.12 & 1.3 & 3.7 & 1.4 & 0.01 & 0.51 & .024 & 4.6 \\
0.17 & 1.6 & 3.7 & 1.3 & 0.00 & 0.51 & .024 & 4.6 \\
0.24 & 1.6 & 7.2 & 1.3 & 0.00 & 0.55 & .043 & 7.7 \\
0.34 & 2.8 & 5.1 & 0.93 & 0.00 & 0.60 & .037 & 6.1 \\
0.44 & 4.6 & 12. & 1.5 & 0.00 & 0.56 & .071 & 13. \\
0.52 & 11.0 & 24. & 1.7 & 0.00 & 0.56 & .15 & 26. \\
\hline
\end{tabular}

\section{A.3 Comparison and final results}

In this section we compare of modeled and measured dilution factors for both spectrometers and all targets. We also suggest the final result to be used in the asymmetry analysis. The central values are obtained from the model. We treat the difference between the model and the experiment as an extra systematic error which, added in quadrature with the model error, gives the uncertainty in the dilution factor. The contribution to the errors in the structure funciton $g_{1}^{n}(x)$ is also provided.

Let us define some measures of agreement between model and experiment. We consider the square of difference per degree of freedom, $\chi^{2}$, between $f_{m}$ in the model and the experimental $f_{\exp }$ weighted by the statistical part of 
the error on measured value $\delta f_{\text {stat }}$ i.e.

$$
\chi^{2}=\frac{\sum_{(x, s p, t g)}\left(\frac{f_{\text {exp }}-f_{m}}{\delta f_{\text {stat }}}\right)^{2}}{\sum_{(x, s p, t g)} 1}
$$

where $(x, s p, t g)$ denotes the $x$ bin, spectrometer and target respectively. Taking the partial sums, we have

$$
\chi^{2}(x)=\frac{\sum_{(s p, t g)}\left(\frac{f_{e x p}-f_{m}}{\delta f_{s t a t}}\right)^{2}}{\sum_{(s p, t g)} 1} .
$$

and similarly $\chi^{2}(\operatorname{tg}), \chi^{2}(s p, t g)$ and $\chi^{2}(x, s p)$.

To average over statistical fluctuations, we form a signed $\eta$ per degree of freedom,

$$
\eta=\frac{\sum_{(x, s p, t g)} \frac{f_{\text {exp }}-f_{m}}{f_{m}} /\left(\delta f_{\text {stat }}\right)^{2}}{\sum_{(x, s p, t g)} \frac{1}{\left(\delta f_{\text {stat }}\right)^{2}}} .
$$

and similarly for $\eta(x), \eta(t g), \eta(s p, t g)$ and $\eta(x, s p)$. We can also form the average weighted by asymmetry error $\delta A_{\|}$,

$$
\eta_{g}=\frac{\sum_{(x, s p, t g)} \frac{f_{e x p}-f_{m}}{f_{m}} /\left(\delta A_{\|}\right)^{2}}{\sum_{(x, s p, t g)} \frac{1}{\left(\delta A_{\|}\right)^{2}}} .
$$


Some feeling about the distribution of the final dilution factor error can be obtained from the straight average,

$$
\delta f f=\frac{\sum_{(x, s p, t g)} \frac{\delta f}{f}}{\sum_{(x, s p, t g)} 1},
$$

and the impact on $g_{1}^{n}$ from the average weighted by asymmetry error $\delta A_{\|}$,

$$
\delta f f_{g}=\frac{\sum_{(x, s p, t g)} \frac{\delta f}{f} /\left(\delta A_{\|}\right)^{2}}{\sum_{(x, s p, t g)} \frac{1}{\left(\delta A_{\|}\right)^{2}}} .
$$

The average $\chi^{2} / n d f$ equals $202 / 171=1.19$ (5\% confidence level, two $\sigma$ away from the expectation value) showing a good agreement between the model and the experiment. Please note that we do not include any systematic errors in the $\chi^{2}$ calculation. One can incorporate them, e.g. by moving the model result within its errors in a way that improves the $\chi^{2}$ distribution. If we scale the model result for each target by less than $1 / 3$ of its error (on average) in the direction suggested by the sign of $\eta$, we find $\chi^{2} / n d f$ equal $156 / 163=0.96$, $64 \%$ confidence level, one $\sigma$ away from the expectation value. That indicates a very good statistical agreement between the model and the experiment.

There is a systematic shift $\eta=+0.59 \%$. The measured dilution factor is slightly greater than the modeled dilution. One could use the experimental value of the dilution in place of the model. That would make the $g_{1}^{n}$ result about $\eta_{g}=-0.24 \%$ smaller on average. The straight average relative error is $\delta f f=6.8 \%$ and the average error on $g_{1}^{n}$ structure function due to the dilution 
is estimated as $\delta f f_{g}=4.9 \%$. The following tables (A.7-A.12) show the same quantities as functions of $x, s p$ and $t g$.
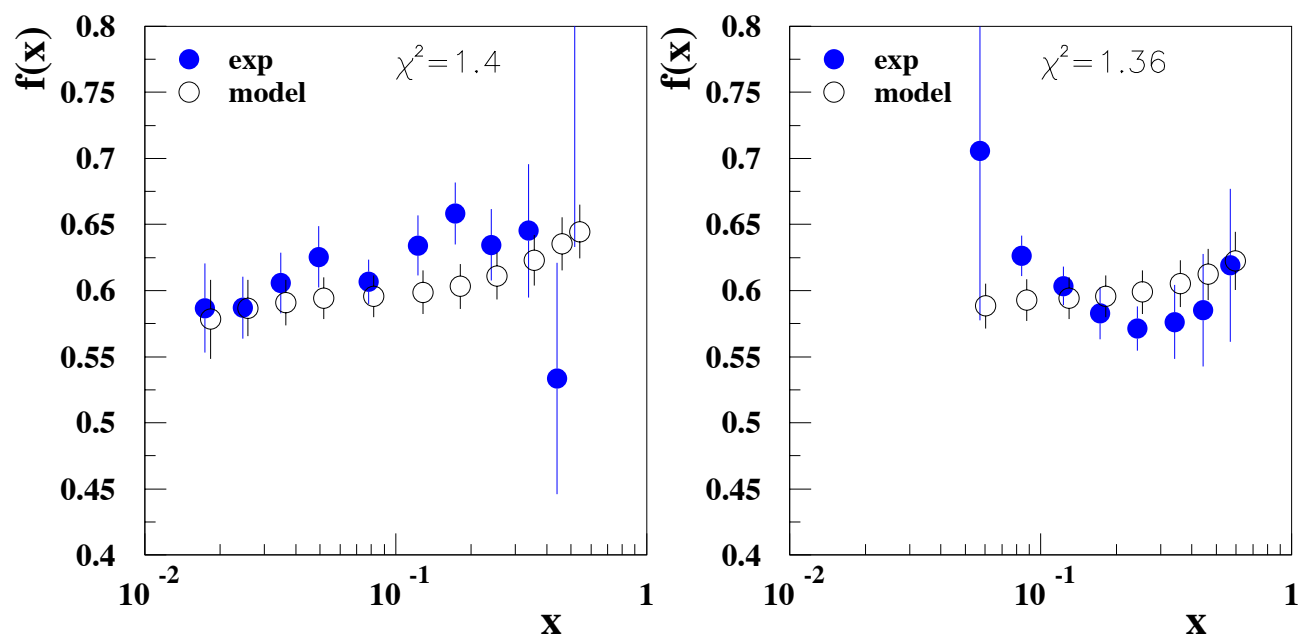

Figure A.3: Target 'Dave', $2.75^{\circ}$ and $5.5^{\circ}$.
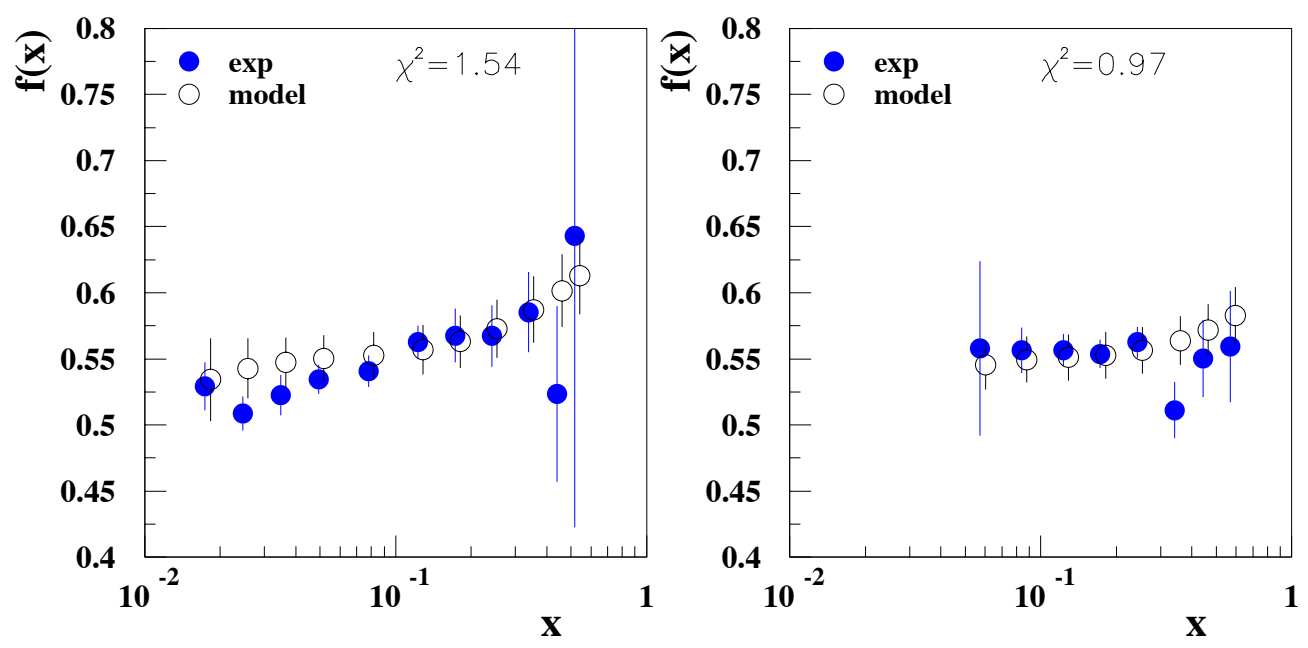

Figure A.4: Target 'Riker', $2.75^{\circ}$ and $5.5^{\circ}$. 


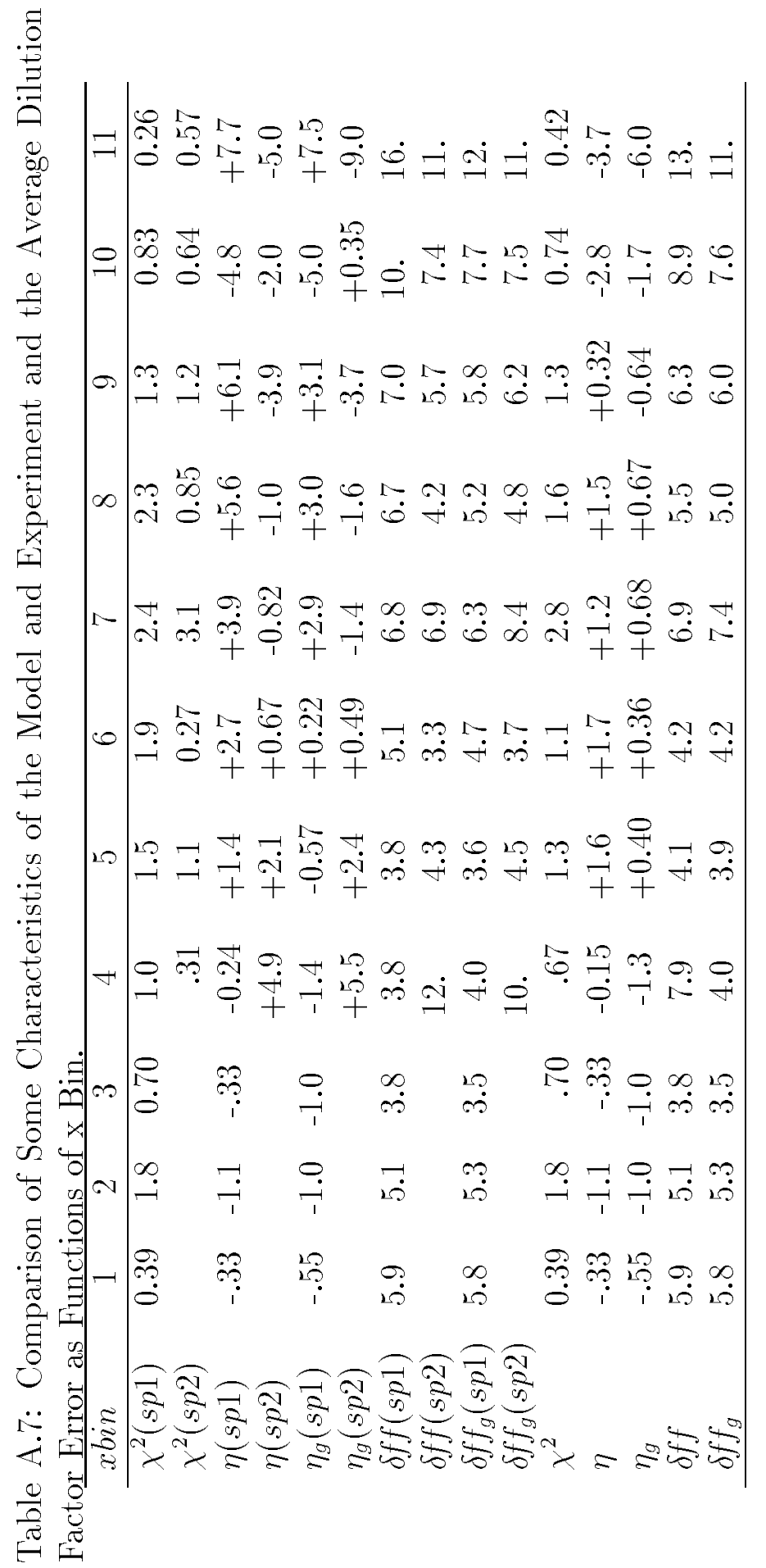


Table A.8: Comparison of Some Characteristics of the Model and Experiment and the Average Dilution Factor Error for Each Target and Spectrometer.

\begin{tabular}{lccccccccc}
\hline$t g$ & 1 & 2 & 3 & 4 & 5 & 6 & 7 & 8 & 9 \\
\hline$\chi^{2}(s p 1)$ & 1.4 & 1.5 & 1.9 & 1.6 & 1.4 & 0.83 & 0.70 & 2.0 & 0.43 \\
$\chi^{2}(s p 2)$ & 1.4 & 0.97 & 0.93 & 0.28 & 0.65 & 2.4 & 1.5 & 0.35 & 0.56 \\
$\eta(s p 1)$ & +3.3 & -2.5 & +3.1 & +1.8 & +2.9 & -2.0 & +1.6 & +2.5 & -0.71 \\
$\eta(s p 2)$ & +0.15 & -0.19 & +3.2 & +0.27 & -1.6 & -4.7 & +7.3 & +0.56 & -3.0 \\
$\eta_{g}(s p 1)$ & +3.4 & -2.2 & +3.3 & +2.3 & +3.1 & -2.0 & +1.8 & +1.8 & -0.69 \\
$\eta_{g}(s p 2)$ & +0.16 & +0.05 & +3.4 & +0.22 & -1.6 & -4.0 & +6.6 & +0.76 & -2.4 \\
$\delta f f(s p 1)$ & 9.3 & 5.8 & 9.5 & 7.3 & 6.4 & 4.7 & 7.6 & 5.7 & 4.7 \\
$\delta f f(s p 2)$ & 6.6 & 4.7 & 6.3 & 4.8 & 3.9 & 7.8 & 15. & 4.9 & 7.2 \\
$\delta f f_{g}(s p 1)$ & 5.2 & 4.9 & 6.7 & 5.2 & 4.1 & 4.6 & 5.4 & 4.9 & 3.9 \\
$\delta f f_{g}(s p 2)$ & 4.5 & 3.9 & 5.6 & 2.8 & 2.6 & 7.4 & 8.1 & 4.1 & 3.9 \\
$f_{g}$ & 0.60 & 0.55 & 0.52 & 0.53 & 0.56 & 0.57 & 0.62 & 0.48 & 0.53 \\
$\chi^{2}$ & 1.4 & 1.3 & 1.5 & 1.1 & 1.1 & 1.5 & 1.0 & 1.3 & 0.49 \\
$\eta$ & 1.8 & -1.6 & +3.1 & +1.2 & +1.4 & -2.6 & +3.0 & +2.2 & -1.0 \\
$\eta_{g}$ & +2.0 & -1.2 & +3.3 & +1.4 & +1.7 & -2.5 & +2.8 & +1.6 & -0.94 \\
$\delta f f$ & 8.2 & 5.3 & 8.1 & 6.2 & 5.4 & 6.0 & 11. & 5.4 & 5.8 \\
$\delta f f_{g}$ & 4.9 & 4.4 & 6.2 & 4.1 & 3.6 & 5.2 & 6.0 & 4.7 & 3.9 \\
\hline
\end{tabular}

Table A.9: Results for 'Dave', 'Riker', 'Bob', 'SMC' and 'Generals', 5.5'.

\begin{tabular}{cccccccccccc}
\hline $\bar{x}$ & \multicolumn{1}{c}{$Q^{2}$} & \multicolumn{1}{c}{$f$} & $\frac{\delta f}{f}$ & $f$ & $\frac{\delta f}{f}$ & $f$ & $\frac{\delta f}{f}$ & $f$ & $\frac{\delta f}{f}$ & $f$ & $\frac{\delta f}{f}$ \\
\hline 0.057 & 4.0 & 0.59 & 20.2 & 0.55 & 4.2 & 0.51 & 9.6 & 0.53 & 14.2 & 0.56 & 6.5 \\
0.084 & 5.5 & 0.59 & 6.2 & 0.55 & 3.5 & 0.52 & 4.3 & 0.53 & 2.8 & 0.57 & 3.2 \\
0.123 & 7.2 & 0.59 & 3.1 & 0.55 & 3.4 & 0.52 & 5.5 & 0.53 & 2.5 & 0.57 & 3.0 \\
0.172 & 8.9 & 0.60 & 3.3 & 0.55 & 3.2 & 0.52 & 9.5 & 0.53 & 2.5 & 0.57 & 6.1 \\
0.242 & 10.7 & 0.60 & 5.4 & 0.56 & 3.4 & 0.52 & 3.8 & 0.54 & 2.6 & 0.57 & 3.2 \\
0.342 & 12.6 & 0.60 & 5.6 & 0.56 & 9.9 & 0.53 & 3.8 & 0.54 & 3.2 & 0.58 & 3.3 \\
0.442 & 13.8 & 0.61 & 5.5 & 0.57 & 5.1 & 0.54 & 9.5 & 0.55 & 7.4 & 0.59 & 6.4 \\
0.568 & 15.0 & 0.62 & 3.6 & 0.58 & 5.5 & 0.55 & 4.7 & 0.56 & 3.7 & 0.60 & 7.7 \\
\hline
\end{tabular}


Table A.10: Results for 'Dave', 'Riker', 'Bob', 'SMC' and 'Generals', $2.75^{\circ}$.

\begin{tabular}{cccccccccccc}
\hline $\bar{x}$ & $\bar{Q}^{2}$ & $f$ & $\frac{\delta f}{f}$ & \multicolumn{1}{c}{$f$} & $\frac{\delta f}{f}$ & \multicolumn{1}{c}{$f$} & $\frac{\delta f}{f}$ & \multicolumn{1}{c}{$f$} & $\frac{\delta f}{f}$ & $f$ & $\frac{\delta f}{f}$ \\
\hline 0.017 & 1.2 & 0.58 & 5.4 & 0.53 & 6.0 & 0.50 & 6.5 & 0.52 & 7.0 & 0.55 & 6.5 \\
0.025 & 1.6 & 0.59 & 3.6 & 0.54 & 7.6 & 0.51 & 4.6 & 0.52 & 3.8 & 0.56 & 5.2 \\
0.035 & 2.0 & 0.59 & 3.8 & 0.55 & 5.7 & 0.52 & 4.6 & 0.53 & 2.9 & 0.56 & 4.0 \\
0.049 & 2.6 & 0.59 & 5.9 & 0.55 & 4.4 & 0.52 & 4.3 & 0.53 & 2.5 & 0.57 & 3.0 \\
0.078 & 3.3 & 0.60 & 3.2 & 0.55 & 3.9 & 0.52 & 6.5 & 0.53 & 2.7 & 0.57 & 4.7 \\
0.123 & 4.0 & 0.60 & 6.5 & 0.56 & 3.6 & 0.52 & 8.4 & 0.54 & 4.2 & 0.57 & 5.2 \\
0.173 & 4.6 & 0.60 & 9.6 & 0.56 & 3.7 & 0.53 & 7.7 & 0.54 & 10.0 & 0.58 & 5.5 \\
0.241 & 5.0 & 0.61 & 4.8 & 0.57 & 4.0 & 0.54 & 12.8 & 0.55 & 12.9 & 0.59 & 6.9 \\
0.339 & 5.4 & 0.62 & 4.7 & 0.59 & 4.3 & 0.55 & 10.0 & 0.57 & 14.3 & 0.60 & 6.4 \\
0.439 & 5.6 & 0.63 & 16.3 & 0.60 & 13.8 & 0.57 & 21.6 & 0.58 & 11.0 & 0.61 & 6.9 \\
0.516 & 5.9 & 0.64 & 38.8 & 0.61 & 6.9 & 0.58 & 17.9 & 0.59 & 8.9 & 0.62 & 24. \\
\hline
\end{tabular}

Table A.11: Results for 'Hermes','Prelims', 'Chance' and 'Picard', 2.75'.

\begin{tabular}{cccccccccc}
\hline $\bar{x}$ & $Q^{2}$ & $f$ & $\frac{\delta f}{f}$ & $f$ & $\frac{\delta f}{f}$ & $f$ & $\frac{\delta f}{f}$ & $f$ & $\frac{\delta f}{f}$ \\
\hline 0.017 & 1.2 & 0.56 & 5.7 & 0.61 & 4.9 & 0.46 & 6.4 & 0.51 & 5.9 \\
0.025 & 1.6 & 0.57 & 6.1 & 0.61 & 7.8 & 0.47 & 4.3 & 0.52 & 3.7 \\
0.035 & 2.0 & 0.57 & 3.3 & 0.62 & 4.2 & 0.48 & 3.8 & 0.53 & 2.9 \\
0.049 & 2.6 & 0.57 & 5.3 & 0.62 & 4.2 & 0.48 & 3.7 & 0.53 & 2.6 \\
0.078 & 3.3 & 0.57 & 3.3 & 0.62 & 4.2 & 0.48 & 4.3 & 0.53 & 2.8 \\
0.123 & 4.0 & 0.58 & 3.5 & 0.63 & 4.9 & 0.48 & 5.0 & 0.54 & 5.2 \\
0.173 & 4.6 & 0.58 & 5.4 & 0.63 & 9.6 & 0.49 & 4.7 & 0.54 & 6.1 \\
0.241 & 5.0 & 0.59 & 3.1 & 0.64 & 4.6 & 0.50 & 8.7 & 0.55 & 3.5 \\
0.339 & 5.4 & 0.60 & 3.2 & 0.65 & 3.5 & 0.51 & 10.6 & 0.57 & 6.5 \\
0.439 & 5.6 & 0.61 & 3.2 & 0.67 & 8.6 & 0.53 & 6.2 & 0.58 & 6.2 \\
0.516 & 5.9 & 0.62 & 10. & 0.68 & 27.4 & 0.54 & 5.4 & 0.59 & 7.2 \\
\hline
\end{tabular}


Table A.12: Results for 'Hermes', 'Prelims', 'Chance' and 'Picard', 5.5'.

\begin{tabular}{crcrcrcccr}
\hline $\bar{x}$ & \multicolumn{1}{c}{$Q^{2}$} & \multicolumn{1}{c}{$f$} & \multicolumn{1}{c}{$\frac{\delta f}{f}$} & \multicolumn{1}{c}{$f$} & \multicolumn{1}{c}{$\frac{\delta f}{f}$} & \multicolumn{1}{c}{$f$} & $\frac{\delta f}{f}$ & \multicolumn{1}{c}{$f$} & $\frac{\delta f}{f}$ \\
\hline \hline 0.057 & 4.0 & 0.57 & 5.6 & 0.62 & 29.8 & 0.47 & 4.1 & 0.52 & 14.4 \\
0.084 & 5.5 & 0.57 & 5.7 & 0.62 & 6.3 & 0.48 & 4.8 & 0.53 & 3.8 \\
0.123 & 7.2 & 0.57 & 4.1 & 0.62 & 4.2 & 0.48 & 3.2 & 0.53 & 2.5 \\
0.172 & 8.9 & 0.57 & 15.0 & 0.62 & 15.6 & 0.48 & 3.9 & 0.53 & 3.4 \\
0.242 & 10.7 & 0.58 & 8.5 & 0.63 & 4.9 & 0.48 & 4.9 & 0.54 & 2.6 \\
0.342 & 12.6 & 0.58 & 3.7 & 0.63 & 6.7 & 0.49 & 3.2 & 0.54 & 12.9 \\
0.442 & 13.8 & 0.59 & 7.4 & 0.64 & 15.2 & 0.50 & 5.2 & 0.55 & 5.0 \\
0.568 & 15.0 & 0.60 & 12.7 & 0.65 & 36.0 & 0.51 & 9.9 & 0.56 & 13.7 \\
\hline
\end{tabular}
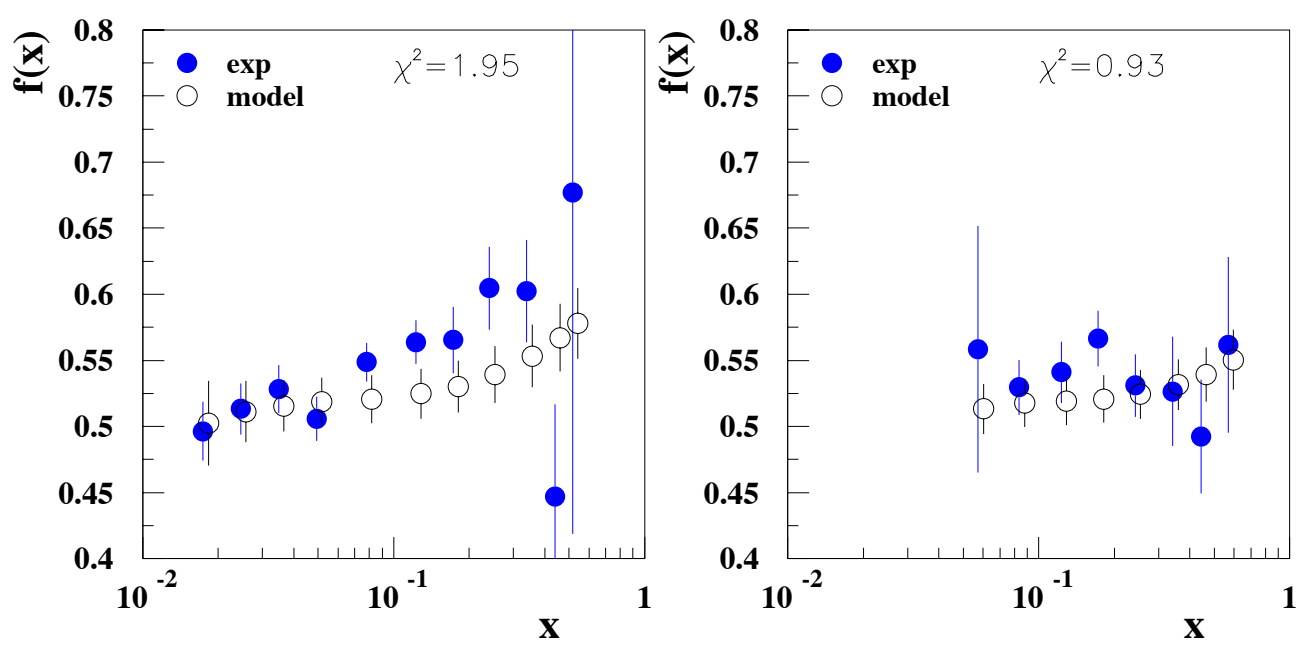

Figure A.5: Target 'Bob', 2.75' and 5.5'. 

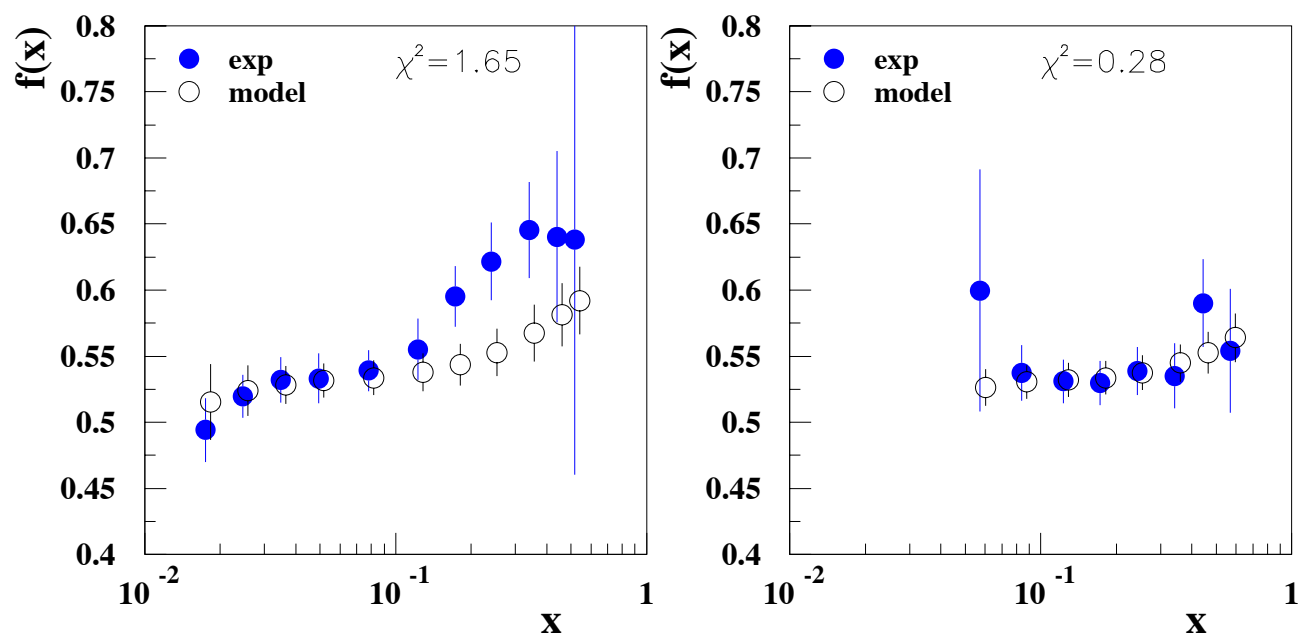

Figure A.6: Target 'SMC', 2.75 ${ }^{\circ}$ and 5.5'.
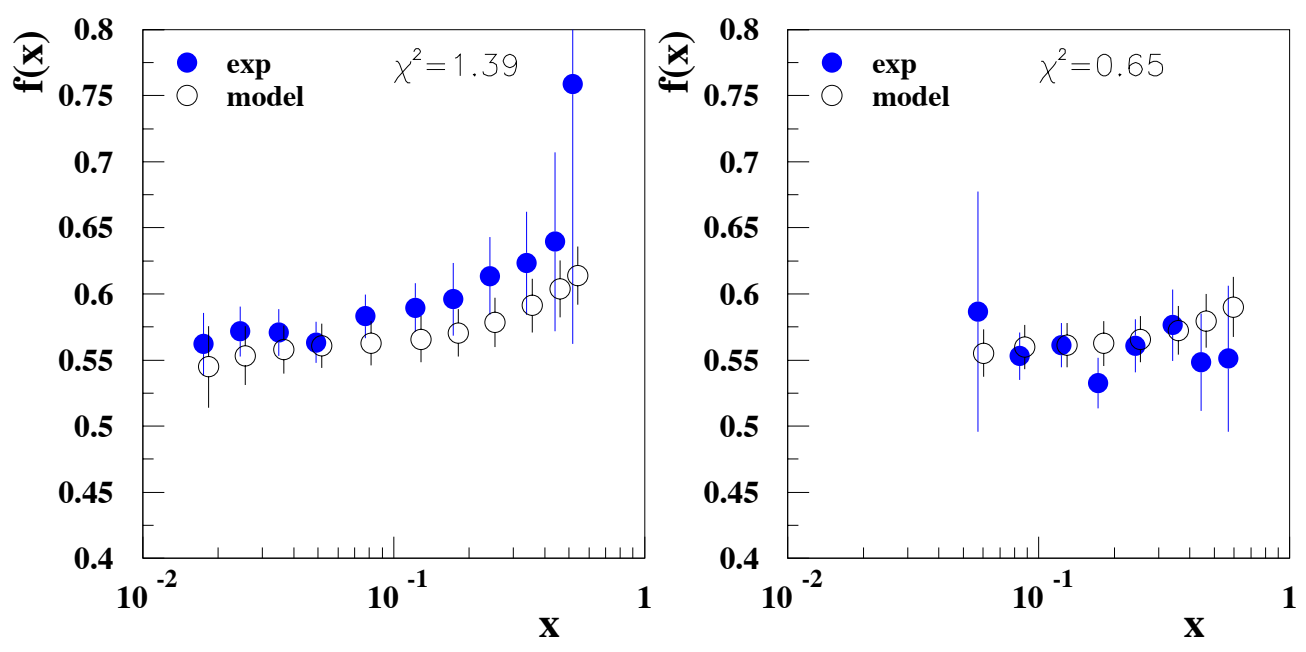

Figure A.7: Target 'Generals', $2.75^{\circ}$ and 5.5 . 

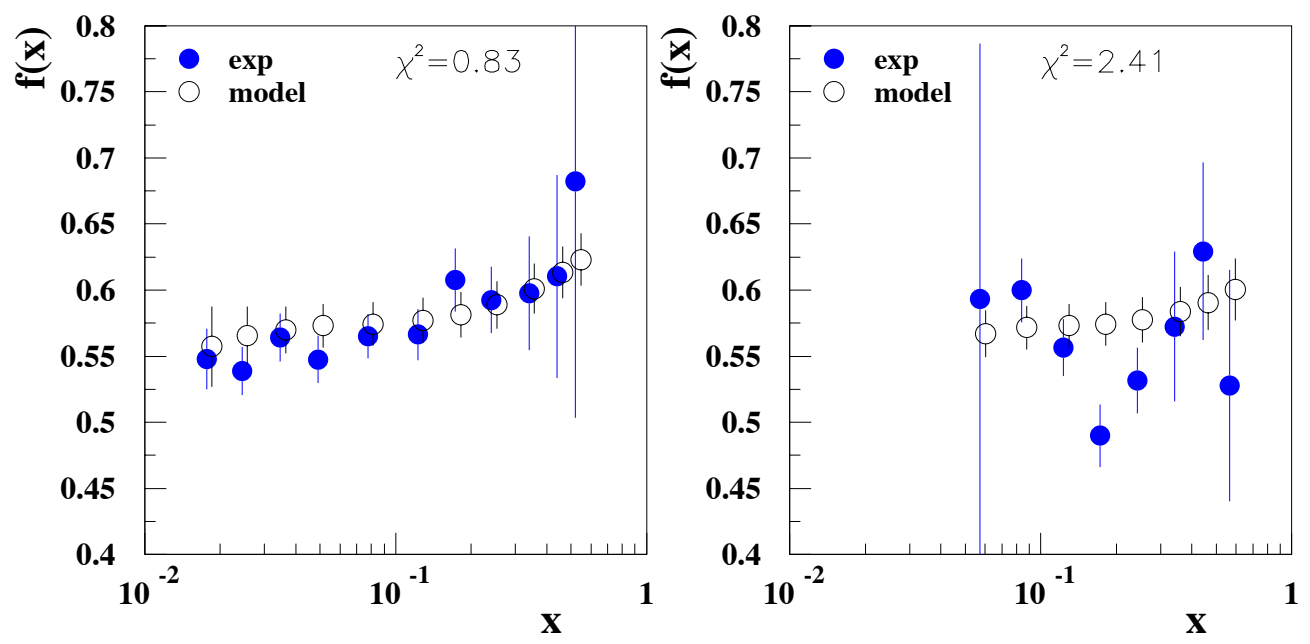

Figure A.8: Target 'Hermes', $2.75^{\circ}$ and $5.5^{\circ}$.
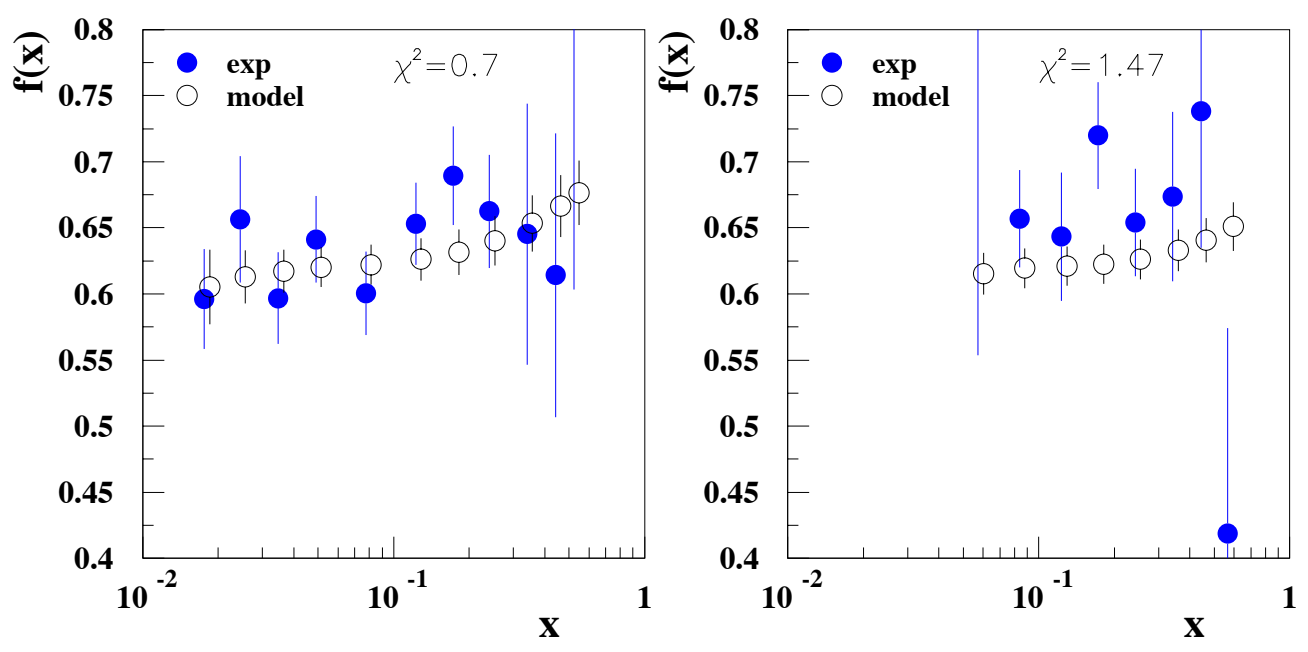

Figure A.9: Target 'Prelims', $2.75^{\circ}$ and $5.5^{\circ}$. 

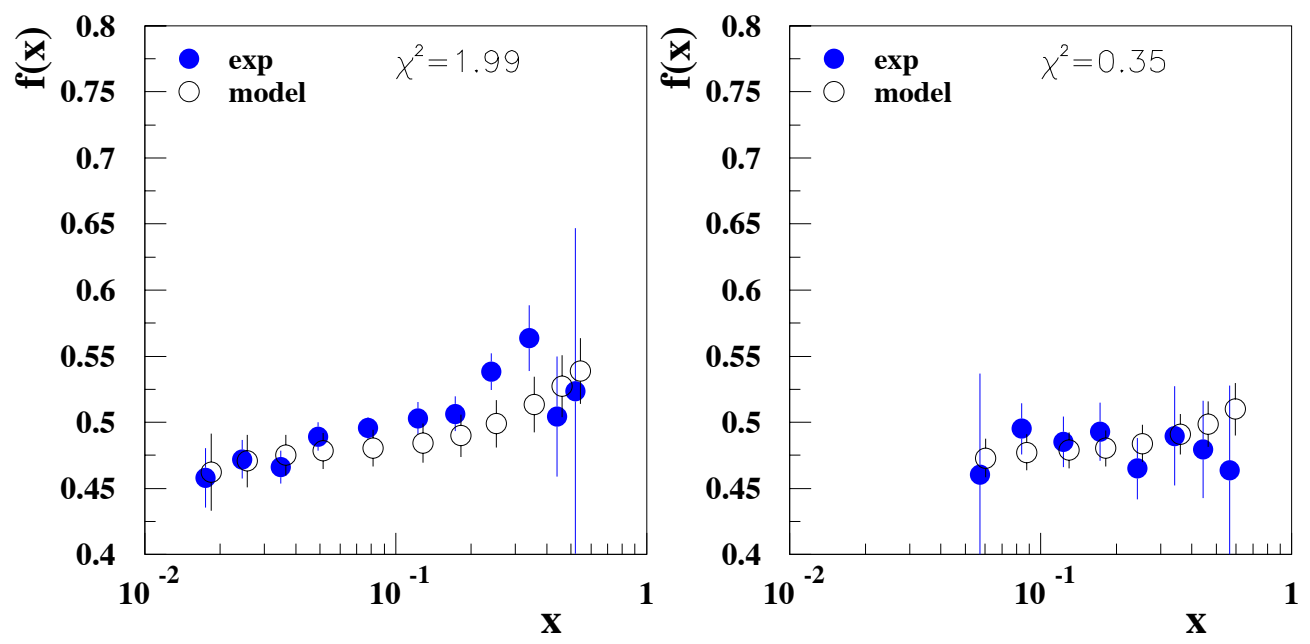

Figure A.10: Target 'Chance', $2.75^{\circ}$ and $5.5^{\circ}$.
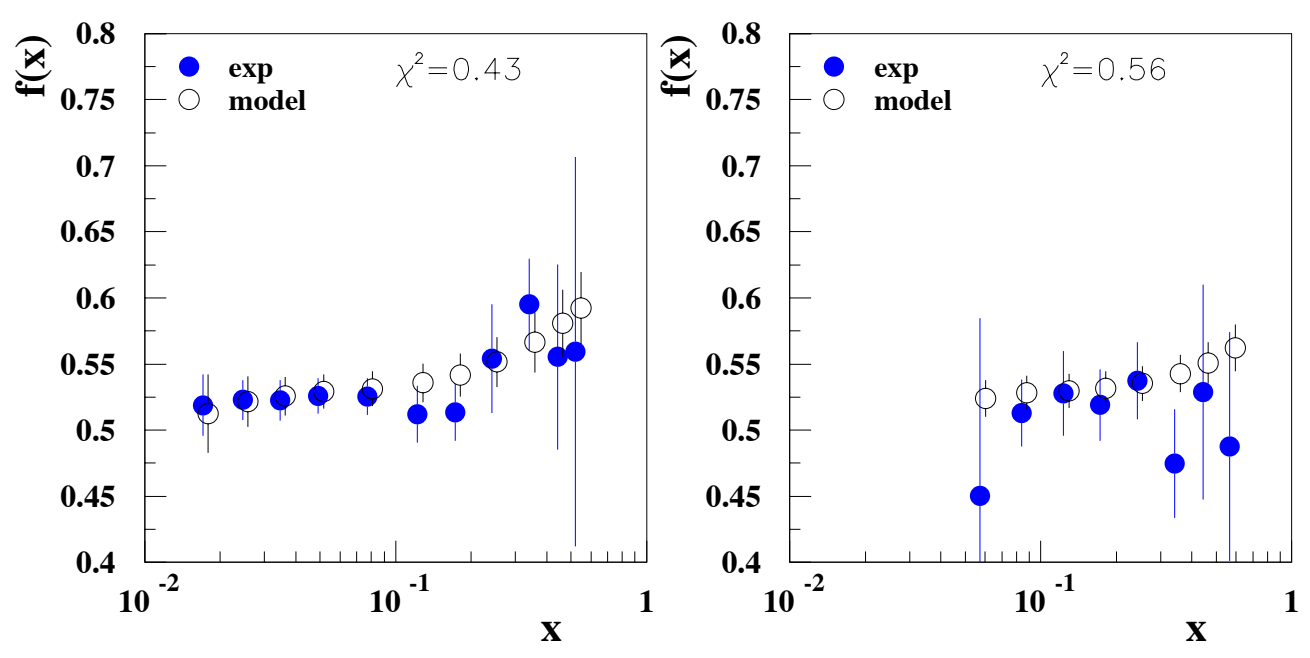

Figure A.11: Target 'Picard', 2.75' and 5.5'. 


\section{APPENDIX B}

\section{NLO DETAILS}

\section{B.1 Wilson coefficients and the splitting functions in the $x$-space}

The polarized coefficient functions have been calculated up to $\mathcal{O}\left(\alpha_{s}^{4}\right)$ [48]. In the next-to-leading order they are expressed as a two-term series

$$
C_{p}\left(x, \alpha_{s}\right)=C_{p}^{(0)}(x)+\frac{\alpha_{s}}{2 \pi} C_{p}^{(1)}(x)
$$

in the strong coupling constant $\alpha_{s}\left(Q^{2}\right)$. The leading order parts $[137,138]$ are consistent with the simple partonic picture,

$$
\begin{aligned}
& C_{q}^{(0)}(x)=\delta(1-x), \\
& C_{G}^{(0)}(x)=0 .
\end{aligned}
$$

The NLO terms have the form:

$$
C_{q}^{(1)}(x)=C_{F}\left[\left(1+x^{2}\right)\left(\frac{\log (1-x)}{1-x}\right)_{+}-\frac{3}{2} \frac{1}{(1-x)_{+}}-\frac{1+x^{2}}{1-x} \log (x)+\right.
$$




$$
\begin{gathered}
\left.+2+x-\left(\frac{9}{2}+\frac{\pi^{2}}{3}\right) \delta(1-x)\right] \\
C_{G}^{(1)}(x)=2 T_{R} N_{f}\left[(2 x-1)\left(\log \left(\frac{1-x}{x}\right)-1\right)+2(1-x)\right],
\end{gathered}
$$

where $C_{F}=4 / 3, T_{R}=1 / 2, N_{f}$ is the number of active flavors and the regularization $1 /(1-x)+$ means

$$
\int_{0}^{1} d x \frac{f(x)}{(1-x)_{+}} \equiv \int_{0}^{1} d x \frac{f(x)-f(1)}{(1-x)},
$$

and $(1-x)_{+}=(1-x)$ for $x<1$. For practical calculations it is useful to use the following version of the above formula:

$$
\int_{x}^{1} \frac{d y}{y} \frac{f(y)}{(1-x / y)_{+}}=f(x) \log \left(\frac{1-x}{x}\right)+\int_{x}^{1} \frac{d y}{y} \frac{f(x)-f(1)}{1-x / y} .
$$

Recently in ref. [130] the splitting functions were computed in the $\overline{\mathrm{MS}}$ scheme in the NLO approximation. They are also expressed as a two-term series in the strong coupling constant $\alpha_{s}\left(Q^{2}\right)$,

$$
P_{i j}(x)=P_{i j}^{(0)}(x)+\frac{\alpha_{s}}{2 \pi} P_{i j}^{(1)}(x) .
$$

We recall (see eq. (4.10), page 180) the LO terms [137, 138],

$$
\begin{aligned}
P_{N S}^{\eta(0)}(x)= & P_{q q}^{(0)}(x)=C_{F}\left[\frac{1+x^{2}}{(1-x)_{+}}+\frac{3}{2} \delta(x-1)\right], \\
P_{q G}^{(0)}(x)= & \frac{1}{2}\left[x^{2}-(1-x)^{2}\right] \\
P_{G q}^{(0)}(x)= & C_{F}\left[\frac{1-(1-x)^{2}}{x}\right], \\
P_{G G}^{(0)}(x)= & C_{A}\left[\left(1+x^{4}\right)\left(\frac{1}{x}+\frac{1}{(1-x)_{+}}\right)-\frac{(1-x)^{3}}{x}+\right. \\
& \left.+\left(\frac{11}{6}-\frac{2}{3} \frac{N_{f} T_{R}}{C_{A}}\right) \delta(x-1)\right]
\end{aligned}
$$


where $C_{A}=3$ and $C_{F}, T_{R}, N_{f}$ and the $1 /(1-x)_{+}$regularization are defined as above. The NLO part is more complicated (note that $\eta^{\text {polarized }}=$ $\left.-\eta^{\text {unpolarized }}\right)$ :

$$
\begin{aligned}
& P_{q q, N S}^{(1), \pm 1}=P_{q q, N S}^{(1), \mp 1 \text { unpolarized }}, \\
& P_{q q}^{(1)}(x)=P_{N S}^{(1)+1}+C_{F} T_{R} N_{f}[2(1-x)-2(1-3 x) \log (x)+ \\
& \left.-2(1+x) \log ^{2}(x)\right] \\
& P_{q G}^{(1)}(x)=C_{F} T_{R} N_{f}[-22+27 x-9 \log (x)+8(1-x) \log (1-x)+ \\
& +\frac{1}{2} \delta p_{q G}(x)\left(4 \log ^{2}(1-x)-8 \log (1-x) \log (x)+2 \log ^{2}(x)+\right. \\
& -8 \zeta(2))]+C_{A} T_{R} N_{f}[2(12-11 x)-8(1-x) \log (1-x)+ \\
& 2(1+8 x) \log (x)-2\left(\log ^{2}(1-x)-\zeta(2)\right) \delta p_{q G}(x)+ \\
& \left.-\left(2 I_{x}-3 \log ^{2}(x)\right) \delta p_{q G}(-x)\right] \text {, } \\
& P_{G q}^{(1)}(x)=C_{F} T_{R} N_{f}\left[-\frac{4}{9}(x+4)-\frac{4}{3} \delta p_{G q}(x) \log (1-x)\right]+ \\
& +C_{F}^{2}\left[-\frac{1}{2}-\frac{1}{2}(4-x) \log (x)-\delta p_{G q}(-x) \log (1-x)\right. \\
& \left.+\left(-4-\log ^{2}(1-x)+\frac{1}{2} \log ^{2}(x)\right) \delta p_{G q}(x)\right] \\
& +C_{A} C_{F}\left[(4-13 x) \log (x)+\frac{1}{3}(10+x) \log (1-x)+\right. \\
& +\frac{1}{9}(41+35 x)+\frac{1}{2}\left(-2 I_{x}+3 \log ^{2}(x)\right) \delta p_{G q}(-x)+ \\
& \left.+\left(\log ^{2}(1-x)-2 \log (1-x) \log (x)-\zeta(2)\right) \delta p_{G q}(x)\right], \\
& P_{G G}^{(1)}(x)=-C_{A} T_{R} N_{f}\left[4(1-x)+\frac{4}{3}(1+x) \log (x)+\frac{20}{9} \delta p_{G G}(x)+\right. \\
& \left.+\frac{4}{3} \delta(1-x)\right]-C_{F} T_{R} N_{f}[10(1-x)+2(5-x) \log (x)+
\end{aligned}
$$




$$
\begin{aligned}
& \left.+2(1+x) \log ^{2}(x)+\delta(1-x)\right]+C_{A}^{2}\left[\frac{1}{3}(29-67 x) \log (x)+\right. \\
& -\frac{19}{2}(1-x)+4(1+x) \log ^{2}(x)-2 I_{x} \delta p_{G G}(-x)+ \\
& +\left(\frac{67}{9}-4 \log (1-x) \log (x)+\log ^{2}(x)-2 \zeta(2)\right) \delta p_{G G}(x)+ \\
& \left.+\left(3 \zeta(3)+\frac{8}{3}\right) \delta(1-x)\right]
\end{aligned}
$$

where

$$
\begin{aligned}
\delta p_{q G}(x) & \equiv 2 x-1 \\
\delta p_{G q}(x) & \equiv 2-x \\
\delta p_{G G}(x) & \equiv \frac{1}{(1-x)_{+}}-2 x+1 .
\end{aligned}
$$

The expression for $P_{q q, N S}^{(1), \pm 1}$ unpolarized is given in ref. [181]. In eqs. (B.14-B.18) $\zeta(2)=\pi^{2} / 6, \zeta(3) \approx 1.202057$ and

$$
I_{x} \equiv \int_{x /(1+x)}^{1 /(1+x)} \frac{d z}{z} \log \left(\frac{1-z}{z}\right) .
$$

Also

$$
I_{x}=-2 L i_{2}(-x)-2 \log (x) \log (1+x)+\frac{1}{2} \log ^{2}(x)-\zeta(2),
$$

where $\mathrm{Li}_{2}(x)$ is the dilogarithm function. By definition

$$
L i_{2}(x)=-\int_{0}^{x} d z \frac{\log (1-z)}{z},
$$

and can be suitably parametrized as [154]

$$
\frac{\operatorname{Li}_{2}(x)}{1+x} \approx 1.010 x-0.846 x^{2}+1.155 x^{3}-1.074 x^{4}+0.550 x^{5} .
$$




\section{B.2 Evolution in the n-th moment Mellin space}

The results given in the previous section B.1 can be equivalently expressed in terms of their moments. This formulation is much more suitable for practical calculations, as argued in section 4.5 (page 195). Let us recall that the nth moment of the function $f(x)$ specified for $x \in(0,1)$ is defined via the Mellin transform

$$
f(n)=\int_{0}^{1} d x x^{n-1} f(x) .
$$

A useful feature of this transformation is that it converts the integral convolution equation,

$$
g_{1}(x) \equiv(C \otimes q)(x)=\int_{x}^{1} \frac{d y}{y} C\left(\frac{x}{y}\right) q(y),
$$

to a simple product

$$
g_{1}(n)=C(n) q(n)
$$

We can apply it to the expression for the structure function

$$
g_{1}\left(x, Q^{2}\right)=\frac{1}{2} \sum_{q}^{N_{f}} e_{q}^{2}\left[C_{q} \otimes(\Delta q+\Delta \bar{q})+\frac{1}{N_{f}} C_{G} \otimes \Delta G\right],
$$

and simplify it to

$$
g_{1}\left(n, Q^{2}\right)=\frac{1}{2} \sum_{q}^{N_{f}} e_{q}^{2}\left[C_{q}(\Delta q+\Delta \bar{q})+\frac{1}{N_{f}} C_{G} \Delta G\right] .
$$

The moments of the Wilson coefficients can be expressed as a series in the strong coupling constant $\alpha_{s}$. In the NLO

$$
C_{i}\left(n, Q^{2}\right)=C_{i}^{(0)}(n)+\frac{\alpha_{s}\left(Q^{2}\right)}{2 \pi} C_{i}^{(1)}(n)
$$


where $i$ in $C_{i}\left(n, Q^{2}\right)$ stands for $q$ or $G$ for quarks or gluons, respectively. The leading order coefficients are

$$
\begin{aligned}
& C_{q}^{(0)}(n)=1, \\
& C_{G}^{(0)}(n)=0,
\end{aligned}
$$

and the NLO coefficients in the $\overline{\mathrm{MS}}$ scheme $[130,131]$ are given by

$C_{q}^{(1)}(n)=C_{F}\left[-S_{2}(n)+\left(S_{1}(n)\right)^{2}+\left(\frac{3}{2}-\frac{1}{n(n+1)}\right) S_{1}(n)+\frac{1}{n^{2}}+\frac{1}{2 n}+\frac{1}{n+1}-\frac{9}{2}\right]$,

$C_{G}^{(1)}(n)=2 T_{f}\left[-\frac{n-1}{n(n+1)}\left(S_{1}(n)+1\right)-\frac{1}{n^{2}}+\frac{2}{n(n+1)}\right]$,

with $C_{F}=4 / 3$ and $T_{f}=N_{f} / 2$ the Casimir invariants for $S U(3)_{\text {flavor }}$, and $N_{f}=3$, the number of flavors. The factors $S_{i}(n)$ will be defined below, eq. (B.51). We note that $C_{q}^{(1)}(n=1)=-(3 / 2) C_{F}$ and $C_{G}^{(1)}(n=1)=0$, so the first moment $\Gamma_{1}\left(Q^{2}\right) \equiv g_{1}\left(n=1, Q^{2}\right)=\int_{0}^{1} d x g_{1}\left(x, Q^{2}\right)$ in eq. (B.26) is

$$
\Gamma_{1}\left(Q^{2}\right)=\frac{1}{2} \sum_{q} e_{q}^{2}\left(1-\frac{\alpha_{s}\left(Q^{2}\right)}{\pi}\right)\left[\Delta q\left(Q^{2}\right)+\Delta \bar{q}\left(Q^{2}\right)\right]
$$

The total gluon helicity $\Delta G\left(Q^{2}\right)$ does not directly couple to $\Gamma_{1}\left(Q^{2}\right)$ due to the vanishing of the integrated gluonic coefficient function in the $\overline{\mathrm{MS}}$ factorization scheme. This vanishing of $C_{G}^{(1)}(1)$, which has been a matter of dispute during the past years $[37,182,183]$, originates from the last term in eq. (B.5) proportional to $2(1-x)$. Since this term derives, when the mass regulator is used, from the soft non-perturbative collinear region [184] where $k_{T}^{2} \sim m_{q}^{2} \ll \Lambda^{2}$, it has been suggested $[161,182,185]$ to absorb it into the definition of the non-perturbative input quark $\Delta q\left(x, Q^{2}=Q_{\circ}^{2}\right)$ and anti-quark 
$\Delta \bar{q}\left(x, Q^{2}=Q_{\circ}^{2}\right)$ distributions. That would change the factorization scheme, e.g. to the Adler-Bardeen scheme, as described in section 4.6 (page 197), which applies to all moments of the coefficient functions as well as to the anomalous dimensions. When the modified dimensional regulator is used, however, the above "soft" term comes from the $k_{T}^{2} \sim \mu_{f}^{2}$ region [186] and hence is genuinely hard. Let us remark again that the physics is independent of a consistent factorization scheme. The scheme dependent interpretation should be clear from the conventions used.

The $Q^{2}$-evolution of the parton distributions is governed by the anomalous dimensions. In the NLO expansion in $\alpha_{s}$

$$
\begin{aligned}
\gamma_{N S}(n) & =\frac{\alpha_{s}}{4 \pi} \gamma_{q q}^{(0)}(n)+\left(\frac{\alpha_{s}}{4 \pi}\right)^{2} \gamma_{N S}^{\eta(1)}(n), \quad \eta= \pm 1 \\
\gamma_{i j}(n) & =\frac{\alpha_{s}}{4 \pi} \gamma_{i j}^{(0)}(n)+\left(\frac{\alpha_{s}}{4 \pi}\right)^{2} \gamma_{i j}^{(1)}(n), \quad i, j \in\{q, G\} .
\end{aligned}
$$

The $n$-dependence will be specified below. It is related to the splitting functions [154] via

$$
\begin{aligned}
& \gamma^{(0)}(n)=-4 P^{(0)}(n), \\
& \gamma^{(1)}(n)=-8 P^{(1)}(n) .
\end{aligned}
$$

The NLO non-singlet (NS) parton densities evolve according to [154, 160]

$$
\begin{aligned}
\Delta q_{N S}^{\eta}\left(n, Q^{2}\right)= & {\left[1+\frac{\alpha_{s}\left(Q^{2}\right)-\alpha_{s}\left(Q_{\circ}^{2}\right)}{4 \pi}\left(\frac{\delta \gamma_{N S}^{\eta(1)}(n)}{2 \beta_{\circ}}-\frac{\beta_{1} \gamma_{q q}^{(0)}(n)}{2 \beta_{\circ}^{2}}\right)\right] . } \\
& \cdot\left(\frac{\alpha_{s}\left(Q^{2}\right)}{\alpha_{s}\left(Q_{\circ}^{2}\right)}\right)^{\gamma_{q q}^{(0)}(n) / 2 \beta_{\circ}} \Delta q_{N S}^{\eta}\left(n, Q_{\circ}^{2}\right)
\end{aligned}
$$

with the input scale $Q_{\circ}^{2}=0.34 \mathrm{GeV}^{2}$. Furthermore, unlike the unpolarized (spin-averaged) parton distributions [154], $\Delta q_{N S}^{\eta=+1}$ corresponds to the valance 
NS combinations

$$
\left.\begin{array}{rl}
\Delta u_{V} & \equiv \Delta u-\Delta \bar{u} \\
\Delta d_{V} & \equiv \Delta d-\Delta \bar{d}
\end{array}\right\} \quad \Delta q_{N S}^{\eta=+1}
$$

while $\Delta q_{N S}^{\eta=-1}$ corresponds to the combinations $\Delta q+\Delta \bar{q}$ appearing in the NS expressions

$$
\left.\begin{array}{rl}
\Delta q_{3} & \equiv(\Delta u+\Delta \bar{u})-(\Delta d+\Delta \bar{d}) \\
\Delta q_{8} & \equiv(\Delta u+\Delta \bar{u})+(\Delta d+\Delta \bar{d})-2(\Delta s+\Delta \bar{s})
\end{array}\right\} \quad \Delta q_{N S}^{\eta=-1} .
$$

We note that the first moments $\Delta q_{N S}^{\eta=-1}(n=1) \equiv \Delta q_{N S}^{-1}$ of the latter $S U(3)_{\text {flavor }}$ diagonal flavor non-singlet combinations do not renormalize, i.e. are independent of $Q^{2}$, due to the conservation of the flavor non-singlet axial vector current, i.e. $\gamma_{q q}^{(0)}(n=1)=\gamma_{N S}^{\eta=-1(1)}(n=1)=0$. The NLO evolution in the flavor singlet sector of

$$
\Gamma\left(n, Q^{2}\right) \equiv\left(\begin{array}{c}
\Delta \Sigma\left(n, Q^{2}\right) \\
\Delta G\left(n, Q^{2}\right)
\end{array}\right)
$$

where $\Delta \Sigma=\Delta u+\Delta d+\Delta s$, is governed by the anomalous dimension $2 \times 2$ matrix

$$
\gamma=\left(\begin{array}{cc}
\gamma_{q q} & \gamma_{q G} \\
\gamma_{G q} & \gamma_{G G}
\end{array}\right)
$$

with the nth moment given by $[154,160]$

$$
\begin{aligned}
\Gamma\left(n, Q^{2}\right)= & \left\{( \frac { \alpha _ { s } ( Q ^ { 2 } ) } { \alpha _ { s } ( Q _ { \circ } ^ { 2 } ) } ) ^ { \lambda _ { - } ^ { n } / ( 2 \beta _ { 0 } ) } \left[P_{-}^{n}-\frac{1}{2 \beta_{\circ}} \frac{\alpha_{s}\left(Q_{\circ}^{2}\right)-\alpha_{s}\left(Q^{2}\right)}{4 \pi} P_{-}^{n} \gamma^{n} P_{-}^{n}+\right.\right. \\
& -\left(\frac{\alpha_{s}\left(Q_{\circ}^{2}\right)}{4 \pi}-\frac{\alpha_{s}\left(Q^{2}\right)}{4 \pi}\left(\frac{\alpha_{s}\left(Q^{2}\right)}{\alpha_{s}\left(Q_{\circ}^{2}\right)}\right)^{\left(\lambda_{+}^{n}-\lambda_{-}^{n}\right) /\left(2 \beta_{0}\right)}\right) . \\
& \left.\left.\cdot \frac{P_{-}^{n} \gamma^{n} P_{+}^{n}}{2 \beta_{\circ}+\lambda_{+}^{n}-\lambda_{-}^{n}}\right]+\left(\lambda_{+}^{n} \leftrightarrow \lambda_{-}^{n}, P_{+}^{n} \leftrightarrow P_{-}^{n}\right)\right\} \Gamma\left(n, Q_{\circ}^{2}\right) .
\end{aligned}
$$


Here

$$
\begin{aligned}
\gamma^{n} & =\gamma^{(1)}(n)-\frac{\beta_{1}}{\beta_{\circ}} \gamma^{(0)}(n), \\
\lambda_{ \pm}^{n} & =\frac{1}{2}\left[\gamma_{q q}^{(0)}(n)+\gamma_{G G}^{(0)}(n) \pm \sqrt{\left(\gamma_{G G}^{(0)}(n)-\gamma_{q q}^{(0)}(n)\right)^{2}+4 \gamma_{q G}^{(0)}(n) \gamma_{G q}^{(0)}(n)}\right] \\
P_{ \pm}^{n} & = \pm\left(\frac{\gamma^{(0)}(n)-\lambda_{\mp}^{n} \mathbb{I}}{\lambda_{+}^{n}-\lambda_{-}^{n}}\right),
\end{aligned}
$$

where $\mathbb{I}$ denotes the $2 \times 2$ identity matrix and $\lambda_{ \pm}^{n}$ the eigenvalues of the 1-loop anomalous dimension matrix $\gamma^{(0)}(n)$.

We use the conventional fixed-flavor scheme [131, 149] and set $N_{f}=3$ in the Wilson coefficients and anomalous dimensions. The experimental data are at relatively low $Q^{2}$ and, although above the pair-production threshold, the heavier quark (charm and bottom) contribution to $g_{1}$ is negligible. The heavy quarks are included in the two-loop running coupling constant,

$$
\frac{\alpha_{s}\left(Q^{2}\right)}{4 \pi}=\frac{1}{\beta_{\circ} \log \left(Q^{2} / \Lambda_{(f)}^{2}\right)}-\frac{\beta_{1}}{\beta_{\circ}^{3}} \frac{\log \left(\log \left(Q^{2} / \Lambda_{(f)}^{2}\right)\right)}{\left(\log \left(Q^{2} / \Lambda_{(f)}^{2}\right)\right)^{2}},
$$

where the coefficients of the $\beta$ function are $\beta_{\circ}=11-2 f / 3$ and $\beta_{1}=102-$ $38 \mathrm{f} / 3$. The number of active flavors $f$ in the coupling $\alpha_{s}\left(Q^{2}\right)$ equals the number of quarks with mass $m_{q}^{2} \leq Q^{2}$. For consistency with the fit to the unpolarized distributions [149] we take $m_{c}=1.5 \mathrm{GeV}, m_{b}=4.5 \mathrm{GeV}$ and $\alpha_{s}\left(M_{Z}^{2}\right)=0.109$ or $\alpha_{s}\left(5 \mathrm{GeV}^{2}\right)=0.237$. The renormalization scales $\Lambda_{(f)}$ are determined by the continuity of $\alpha_{s}$ at the quark mass threshold, $\alpha_{s}\left(m_{q}^{2}, f\right)=\alpha_{s}\left(m_{q}^{2}, f+1\right)$, which gives

$$
\Lambda_{(3,4,5)}^{\overline{\mathrm{MS}}}=248,200,131 \mathrm{MeV}
$$


Although $\alpha_{s}$ is continuous across the quark thresholds, the $\beta$ coefficients, which depend explicitly on the number of active flavors $f$ only, are not. Due to this the evolution is done in steps: If $m_{c}^{2}<Q^{2}<m_{b}^{2}$ we first evolve the parton densities from $Q_{\circ}^{2}$ to $Q^{2}=m_{c}^{2}$ with $f=3$ and then from the charm threshold $m_{c}^{2}$ to $Q^{2}$ (or to $m_{b}^{2}$ and then to $Q^{2}$ if $Q^{2}>m_{b}^{2}$ ).

Dropping all first order terms $\left(\beta_{1}, \gamma^{(1)}, C_{q, G}^{(1)}\right)$ in all above equations would give the leading-order results.

Having obtained the moments of the parton densities, we multiply them by the Wilson coefficients to obtain the moments of the structure function $g_{1}(n)$. Now we apply the inverse Mellin transform [153] in the complex $n$ space to obtain $x$ dependence,

$$
g_{1}(x)=\frac{1}{2 \pi i} \int_{c-i \infty}^{c+i \infty} d n x^{-n} g_{1}(n) .
$$

The real number $c$ is chosen so that the integral $\int_{c-i \infty}^{c+i \infty} d n x^{-n} g_{1}(n)$ is absolutely convergent, i.e. $c$ lies to the right to all singularities of $g_{1}(n)$. The contour of the integration in eq. (B.42) is displayed in fig. B.1 and denoted by $\mathrm{C}_{0}$. Also shown is a deformed route $\mathbf{C}_{1}$, which yields the same result as long as no singularities $n_{i}$ of $g_{1}(n)$ are enclosed by the region $\mathbf{C}_{0}-\mathbf{C}_{1}$. This is the case for the NLO evolution of the structure functions, where all the singularities $n_{i}$ (denoted by crosses in fig. B.1) are real with $n_{i}<n_{\max }<c$. Converting to integration over a real variable we rewrite eq. (B.42) as

$$
g_{1}(x)=\frac{1}{\pi} \int_{0}^{\infty} d z \operatorname{Im}\left[x^{-c-z \exp (i \phi)} g_{1}(n=c+z \exp (i \phi))\right]
$$

Obviously this integral does not depend on $c$ or $\phi$. We chose $c=2.1$ and 


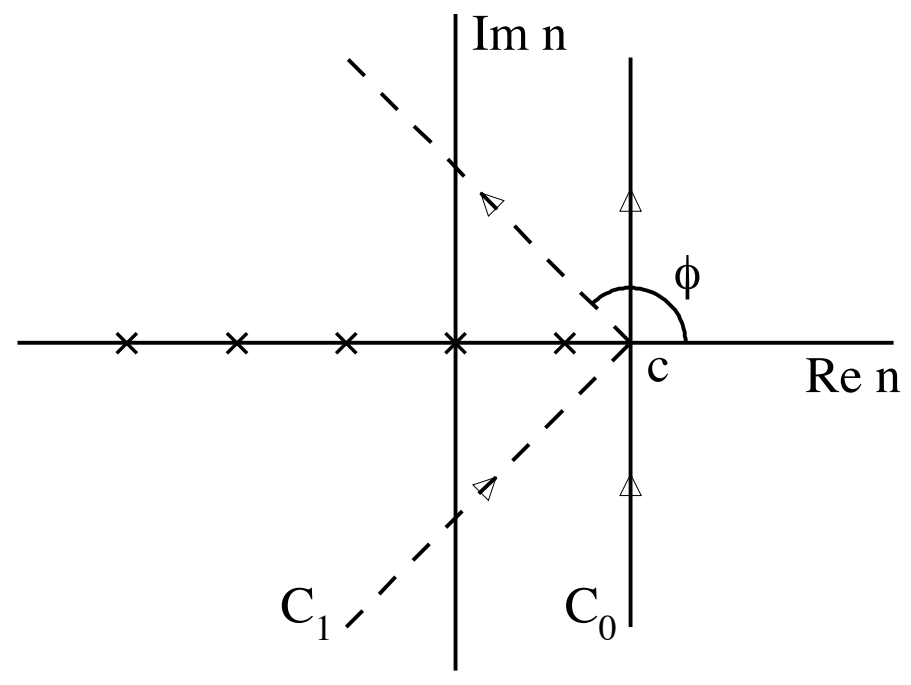

Figure B.1: Integration contours of the Mellin inverse transform. The crosses denote schematically singularities of $g_{1}^{n}$.

$\phi=1.7 \mathrm{rad}$ and set the upper limit of the integration in eq. (B.43) to 9. These parameters give stable results down to $x \geq 10^{-4}$. In the numerical computations the integral in eq. (B.43) was approximated by 24-point Gauss-Legendre quadrature formulas [187] using the CERN Program Library routine RGQUAD [188].

The spin-dependent anomalous dimensions in the leading order were originally calculated in $[138,155]$ and are given by

$$
\begin{aligned}
\gamma_{q q}^{(0)}(n) & =4 C_{F}\left[2 S_{1}(n)-\frac{1}{n(n+1)}-\frac{3}{2}\right], \\
\gamma_{q G}^{(0)}(n) & =-8 T_{f} \frac{n-1}{n(n+1)}, \\
\gamma_{G q}^{(0)}(n) & =-4 C_{F} \frac{n+2}{n(n+1)},
\end{aligned}
$$




$$
\begin{aligned}
\gamma_{G G}^{(0)}(n) & =4 C_{A}\left[2 S_{1}(n)-\frac{4}{n(n+1)}-\frac{11}{6}\right]+\frac{8}{3} T_{f}, \\
\gamma_{N S}^{\eta(0)}(n) & =\gamma_{q q}^{(0)}(n), \quad \eta= \pm 1
\end{aligned}
$$

where $C_{F}=4 / 3, C_{A}=3$ and $T_{f}=N_{f} / 2$ and $N_{f}=3$. Note that $\gamma_{N S}^{\eta(0)}=$ $\gamma_{q q}^{(0)}=\gamma_{q q}^{(0) \text { unp }}$, where the latter quantity refers to the spin-averaged (unpolarized) anomalous dimension. Furthermore, for the first $n=1$ moment we have $\gamma_{q q}^{(0)}(n=1)=\gamma_{q G}^{(0)}(n=1)=0$ as a consequence of helicity conservation at the quark-gluon vertex. Thus the first moments $\Delta q_{N S}^{\eta}, \Delta \Sigma$ of quark distributions (see eq. (B.34), (B.39)) are conserved (i.e. $Q^{2}$ independent) in the leading order.

The two-loop NLO $(\overline{\mathrm{MS}})$ non-singlet anomalous dimensions $\gamma_{N S}^{\eta(1)}(n)$ are the same as found in the unpolarized case [189], $\gamma_{N S}^{\eta(1)}(n)=\gamma_{N S}^{\eta(1) \text { unp }}(n)$,

$$
\begin{aligned}
& \gamma_{N S}^{\eta(1)}(n)=\quad C_{F}^{2} \quad\left[\frac{16 S_{1}(n)(2 n+1)}{n^{2}(n+1)^{2}}+24 S_{2}(n)+64 \tilde{S}(n, \eta)-8 S_{3}^{\prime}\left(\frac{n}{2}, \eta\right)-\right. \\
& 3+16\left(2 S_{1}(n)-\frac{1}{n(n+1)}\right)\left(S_{2}(n)-S_{2}^{\prime}\left(\frac{n}{2}, \eta\right)\right)- \\
& \left.8 \frac{3 n^{3}+n^{2}-1}{n^{3}(n+1)^{3}}-16 \eta \frac{2 n^{2}+2 n+1}{n^{3}(n+1)^{3}}\right]+ \\
& C_{F} C_{A} \quad\left[\frac{536}{9} S_{1}(n)-8\left(2 S_{1}(n)-\frac{1}{n(n+1)}\right)\left(2 S_{2}(n)-\right.\right. \\
& \left.S_{2}^{\prime}\left(\frac{n}{2}, \eta\right)\right)-\frac{88}{3} S_{2}(n)-32 \tilde{S}(n, \eta)+4 S_{3}^{\prime}\left(\frac{n}{2}, \eta\right)-\frac{17}{3}- \\
& \frac{4}{9} \frac{151 n^{4}+236 n^{3}+88 n^{2}+3 n+18}{n^{3}(n+1)^{3}}+ \\
& \left.8 \eta \frac{2 n^{2}+2 n+1}{n^{3}(n+1)^{3}}\right]+ \\
& C_{F} T_{f} \quad\left[-\frac{160}{9} S_{1}(n)+\frac{32}{3} S_{2}(n)+\frac{4}{3}+\frac{16}{9} \frac{11 n^{2}+5 n-3}{n^{2}(n+1)^{2}}\right] \text {. }
\end{aligned}
$$


We note that $\gamma_{N S}^{\eta=-1(1)}(n=1)=0$ so the first moments of $\eta=-1 \mathrm{SU}(3)_{\text {flavor }}$ non-singlet distributions, $\Delta q_{3}$ and $\Delta q_{8}$, are conserved, i.e. are independent of $Q^{2}$ (the conservation of the flavor non-singlet axial vector current). The NLO flavor singlet anomalous dimensions $\gamma_{i j}^{(1)}(n)$ in the $\overline{\mathrm{MS}}$ scheme are as follows $[130,131]$ :

$$
\gamma_{q q}^{(1)}(n)=\gamma_{N S}^{\eta=-1(1)}(n)+16 C_{F} T_{f} \frac{n^{4}+2 n^{3}+2 n^{2}+5 n+2}{n^{3}(n+1)^{3}},
$$

where $\gamma_{N S}^{\eta=-1(1)}(n)$ is given by eq. (B.45), and

$$
\begin{aligned}
& \gamma_{q G}^{(1)}(n)=8 C_{F} T_{f} \quad\left[2 \frac{n-1}{n(n+1)}\left(S_{2}(n)-S_{1}^{2}(n)\right)+4 \frac{n-1}{n^{2}(n+1)} S_{1}(n)\right. \\
& \left.-\frac{5 n^{5}+5 n^{4}-10 n^{3}-n^{2}+3 n-2}{n^{3}(n+1)^{3}}\right] \\
& +16 C_{A} T_{f} \quad\left[\frac{n-1}{n(n+1)}\left(-S_{2}(n)+S_{2}^{\prime}\left(\frac{n}{2},-1\right)+S_{1}^{2}(n)\right)\right. \\
& -\frac{4}{n(n+1)^{2}} S_{1}(n) \\
& \left.-\frac{n^{5}+n^{4}-4 n^{3}+3 n^{2}-7 n-2}{n^{3}(n+1)^{3}}\right] \text {, } \\
& \gamma_{G q}^{(1)}(n)=32 C_{F} T_{f} \quad\left[-\frac{n+2}{3 n(n+1)} S_{1}(n)+\frac{5 n^{2}+12 n+4}{9 n(n+1)^{2}}\right] \\
& +\quad 4 C_{F}^{2} \quad\left[2 \frac{n+2}{n(n+1)}\left(S_{2}(n)+S_{1}^{2}(n)\right)-2 \frac{3 n^{2}+7 n+2}{n(n+1)^{2}} S_{1}(n)\right. \\
& \left.+\frac{9 n^{5}+30 n^{4}+24 n^{3}-7 n^{2}-16 n-4}{n^{3}(n+1)^{3}}\right] \\
& +8 C_{A} C_{F} \quad\left[\frac{n+2}{n(n+1)}\left(-S_{2}(n)+S_{2}^{\prime}\left(\frac{n}{2},-1\right)-S_{1}^{2}(n)\right)\right. \\
& +\frac{11 n^{2}+22 n+12}{3 n^{2}(n+1)} S_{1}(n) \\
& \left.-\frac{76 n^{5}+271 n^{4}+254 n^{3}+41 n^{2}+72 n+36}{9 n^{3}(n+1)^{3}}\right] \text {, }
\end{aligned}
$$




$$
\begin{aligned}
\gamma_{G G}^{(1)}(n)=8 C_{F} T_{f} & \frac{n^{6}+3 n^{5}+5 n^{4}+n^{3}-8 n^{2}+2 n+4}{n^{3}(n+1)^{3}} \\
+\quad 32 C_{A} T_{f} & {\left[-\frac{5}{9} S_{1}(n)+\frac{3 n^{4}+6 n^{3}+16 n^{2}+13 n-3}{9 n^{2}(n+1)^{2}}\right] \quad \text { B.49) } } \\
+\quad 4 C_{A}^{2} & {\left[-S_{3}^{\prime}\left(\frac{n}{2},-1\right)-4 S_{1}(n) S_{2}^{\prime}\left(\frac{n}{2},-1\right)\right.} \\
& +8 \tilde{S}(n,-1)+\frac{8}{n(n+1)} S_{2}^{\prime}\left(\frac{n}{2},-1\right) \\
& +2 \frac{67 n^{4}+134 n^{3}+67 n^{2}+144 n+72}{9 n^{2}(n+1)^{2}} S_{1}(n) \\
& \left.-\frac{48 n^{6}+144 n^{5}+469 n^{4}+698 n^{3}+7 n^{2}+258 n+144}{9 n^{3}(n+1)^{3}}\right] .
\end{aligned}
$$

where

$$
\begin{aligned}
S_{k}(n) & \equiv \sum_{j=1}^{n} \frac{1}{j^{k}} \\
S_{k}^{\prime}\left(\frac{n}{2}, \eta\right) & \equiv 2^{k-1} \sum_{j=1}^{n} \frac{1+(-1)^{j}}{j^{k}} \\
& =\frac{1}{2}(1+\eta) S_{k}\left(\frac{n}{2}\right)+\frac{1}{2}(1-\eta) S_{k}\left(\frac{n-1}{2}\right) \\
\tilde{S}(n, \eta) & \equiv \sum_{j=1}^{n} \frac{(-1)^{j}}{j^{2}} S_{1}(j) \\
& =-\frac{5}{8} \zeta(3)+\eta\left[\frac{S_{1}(n)}{n^{2}}+\frac{\zeta(2)}{2} G(n)+\int_{0}^{1} d x x^{n-1} \frac{\mathrm{Li}_{2}(x)}{1+x}\right]
\end{aligned}
$$

with $G(n) \equiv \psi\left(\frac{n+1}{2}\right)-\psi\left(\frac{n}{2}\right)$ and $\eta= \pm 1$ for $\gamma_{N S}^{\eta= \pm 1(1)}(n)$ and $\eta=-1$ for the flavor singlet anomalous dimensions $\gamma_{i j}^{(1)}(n)$. The dilogarithm function $L i_{2}(x)$ is defined as $L i_{2}(x)=-\int_{0}^{x} d z \log (1-z) / z$. We note that $\gamma_{N S}^{\eta=-1(1)}(n=$ $1)=0, \gamma_{q q}^{\eta=-1(1)}(n=1)=24 C_{F} T_{f}$ and $\gamma_{q G}^{\eta=-1(1)}(n=1)=0$. The analytical continuations in complex $n$, required for the inverse Mellin transform of these 
sums to $x$ space, are well known [154]:

$$
\begin{array}{lll}
S_{1}(n)=\gamma_{E}+\psi(n+1), & & \gamma_{E}=0.577216 \\
S_{2}(n)=\zeta(2)-\psi^{\prime}(n+1), & \zeta(2)=\frac{\pi^{2}}{6} \\
S_{3}(n)=\zeta(3)+\frac{1}{2} \psi^{\prime \prime}(n+1), & \zeta(3)=1.202057
\end{array}
$$

where $\psi^{(k)}(n)=d^{(k+1)} \log (\Gamma(n)) / d n^{(k+1)}$. These $\psi^{(k)}(n)$ functions can be approximated, for $\operatorname{Re}(n) \geq 10$, by their asymptotic expansions

$$
\begin{aligned}
\psi(n) & \approx \log (n)-\frac{1}{2 n}-\frac{1}{12 n^{2}}+\frac{1}{120 n^{4}}-\frac{1}{256 n^{6}} \\
\psi^{\prime}(n) & \approx \frac{1}{n}+\frac{1}{2 n^{2}}+\frac{1}{6 n^{3}}-\frac{1}{30 n^{5}}+\frac{1}{42 n^{7}}-\frac{1}{30 n^{9}} \\
\psi^{\prime \prime}(n) & \approx-\frac{1}{n^{2}}-\frac{1}{n^{3}}-\frac{1}{2 n^{4}}+\frac{1}{6 n^{6}}-\frac{1}{6 n^{8}}+\frac{3}{10 n^{10}}-\frac{5}{6 n^{12}}
\end{aligned}
$$

For $\operatorname{Re}(n)<10$ one can use the recursion relation

$$
\psi^{(k)}(n+1)=\psi^{(k)}(n)+\frac{(-1)^{k} k !}{n^{k+1}}
$$

in order to reach $\psi^{(k)}(n)$ with $\operatorname{Re}(n) \geq 10$. The integral with the dilogarithm function present in the last of equations (B.50) can be computed by taking into account the $L i_{2}(x)$ expansion given by eq. (B.21),

$$
\int_{0}^{1} d x x^{n-1} \frac{L i_{2}(x)}{1+x} \approx \frac{1.010}{n+1}-\frac{0.846}{n+2}+\frac{1.155}{n+3}-\frac{1.074}{n+4}+\frac{0.550}{n+5} .
$$

The initial partonic distributions at the $Q_{\circ}^{2}$ scale can be written as the series in $x^{\alpha}(1-x)^{\beta}$, see eq. $(4.42),(4.43)$ in chapter 4 on page 191. Their Mellin transform can be easily obtained in terms of the Euler's beta function,

$$
B(w, v)=B(v, w)=\int_{0}^{1} d x x^{v-1}(1-x)^{w-1},
$$


which is simply related to the gamma function,

$$
B(w, v)=\frac{\Gamma(w) \Gamma(v)}{\Gamma(v+w)}
$$

For computations we used the complex $\log (\Gamma(z))$ function CLGAMA of the CERN Program Library [190].

The evolution and fitting codes were optimized for speed. The most time consuming computation was calculation of the anomalous dimensions. We thus used, as mentioned before, fixed-point (24 of them) integration via Gauss quadratures so that the anomalous dimensions had to be obtained once per each of 24 complex $n$-points. The matrices used in the singlet evolution, eq. (B.39), were calculated once for each active flavor $f$. The moments of the initial distributions were recalculated once for each set of fit parameters. A typical fit with 8 free parameters to 168 points (in the range $0.005 \leq x \leq 0.75$ and $\left.1 \mathrm{GeV}^{2} \leq \mathrm{Q}^{2} \leq 58 \mathrm{GeV}^{2}\right)$ took $\sim 4$ min on a DEC Alpha 600 5/266 computer, which corresponds to 800-1000 iterations in MINUIT [165].

To test our code we first considered the leading order direct integration in the Bjorken $x$ space $[191,192]$, evolving the initial distributions in the small $Q^{2}$ steps, see eq. (4.5), (4.6), (4.10), page 179. Than we developed the NLO code using the Mellin transform technique and checked that the results obtained in its $\mathrm{LO}$ mode were the same as those from the direct $x$ space evolution. Next we compared the NLO evolution against the 'standard' parametrization of ref. [131]. We evolved the distributions from the initial scale $Q_{\circ}^{2}=0.34 \mathrm{GeV}^{2}$ up to $100 \mathrm{GeV}^{2}$ to see the excellent agreement with the 
output of the GRSV code provided by one of the authors [193] of ref. [131]. The comparison is shown in fig. B.2.
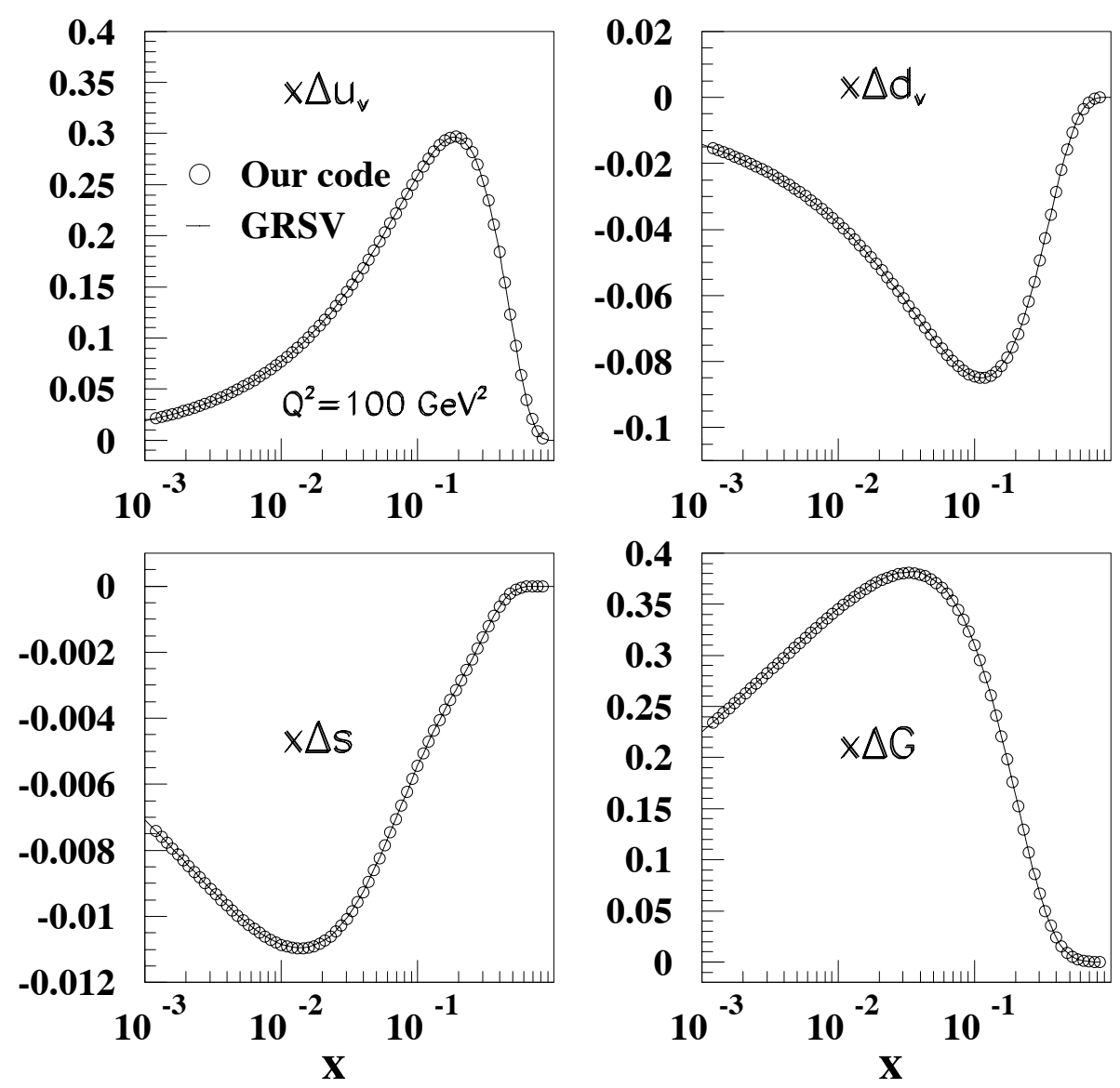

Figure B.2: Comparison of NLO partonic distributions evolved from $Q_{\circ}^{2}=$ $0.34 \mathrm{GeV}^{2}$ to $Q^{2}=100 \mathrm{GeV}^{2}$ with the output of the GRSV code $[131,193]$. 